Supporting Information for

\title{
Substituent Directed Photo-Transformations of BN-Heterocycles: Elimination vs Isomerization via Selective B-C Bond Cleavage
}

Deng-Tao Yang, Soren K. Mellerup, Jin-Bao Peng, Xiang Wang, Quan-Song Li, * and Suning Wang*

I. General procedure.

II. Syntheses of BN-heterocycles.

III. Investigation of photoreactivity of BN-heterocycles.

IV. Photophysical properties.

V. NMR data.

VI. Computational results.

VII. X-ray crystallographic analyses.

VIII. References. 


\section{General Procedure}

All solvents were freshly distilled over sodium metal and stored under nitrogen prior to use. All starting materials were purchased from Sigma-Aldrich Chemicals Corp. except 1,3,5Tris(trifluoromethyl)benzene, which was purchased from TCI America Co. All NMR spectra $\left({ }^{1} \mathrm{H},{ }^{13} \mathrm{C}\right.$,

${ }^{11} \mathrm{~B},{ }^{19} \mathrm{~F}, \mathrm{COSY}$, NOESY, HMQC, HMBC) were recorded on Bruker Avance 300, 400, $500 \mathrm{MHz}$ spectrometers. UV-Vis and Fluorescence measurements were made on a Varian Cary 50 UV-Vis spectrophotometer and Photon Technologies International Quanta Master Model C-60 spectrometer respectively. Solution quantum yields were calculated using optically dilute solutions $(\mathrm{A} \approx 0.1)$ relative to 9,10-diphenylanthracene. ${ }^{1}$ High-resolution mass spectra (HRMS) were obtained from an Applied Biosystems Qstar XL spectrometer. The ligand used to prepare BN-3 was synthesized according to the literature procedure. ${ }^{2}$

\section{Synthesis of BN-Heterocycles}

\section{a. Synthesis of BN-Heterocycle BN-1}
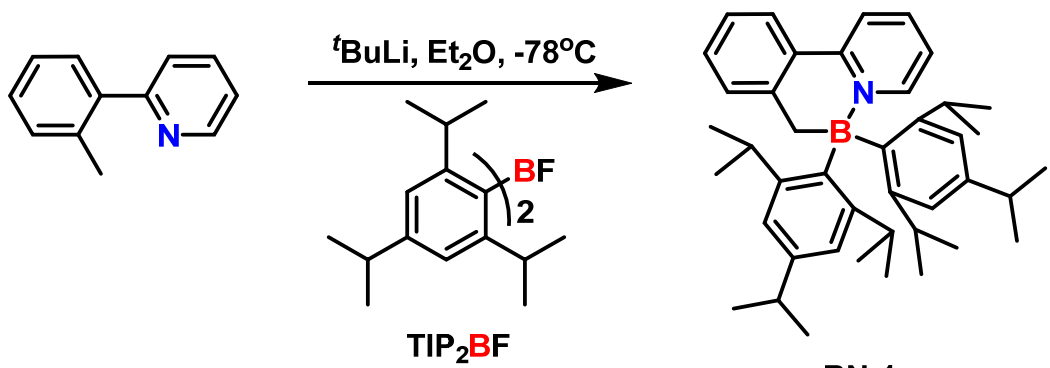

BN-1

Preparation of $\mathrm{TIP}_{2} \mathrm{BF}:{ }^{3}$ A two-necked $50 \mathrm{ml}$ round-bottomed flask equipped with reflux condenser was charged with magnesium turnings $(158 \mathrm{mg}, 6.6 \mathrm{mmol})$, 2,4,6-triisopropylphenyl bromide (1.86 g, 6.6 mmol), THF (30 mL), and one crystal of iodine. The mixture was warmed to initiate the reaction and then heated under reflux overnight. Upon cooling to room temperature, freshly distilled $\mathrm{BF}_{3}$. $\mathrm{OEt}_{2}(393$ ul, $3.2 \mathrm{mmol}$ ) was added to the pre-cooled Grignard solution of TripMgBr at $-78^{\circ} \mathrm{C}$. Once addition was complete, the coolant was removed and the mixture was stirred at room temperature for 30 minutes, 3 hours under reflux, then $2 \mathrm{~h}$ at room temperature. This solution of $\mathrm{TIP}_{2} \mathrm{BF}$ was used directly in next step.

t-BuLi $(2.7 \mathrm{~mL}, 1.7 \mathrm{M}$ in hexane, $4.66 \mathrm{mmol})$ was added to a solution of 2-(o-tolyl)pyridine $(358 \mathrm{mg}$, $2.12 \mathrm{mmol})$ in $\mathrm{Et}_{2} \mathrm{O}(15 \mathrm{~mL})$ at $-78^{\circ} \mathrm{C}$. After stirring at this temperature for $1 \mathrm{~h}$, the pre-cooled boron reagent $\mathrm{TIP}_{2} \mathrm{BF}$ was added via cannula transfer with vigorous stirring at $-78{ }^{\circ} \mathrm{C}$. The mixture was stirred for another hour at $-78{ }^{\circ} \mathrm{C}$, then allowed to warm slowly to room temperature and stirred overnight. The solution was extracted with $\mathrm{CH}_{2} \mathrm{Cl}_{2}$ and water. The organic phase was dried over magnesium sulfate and filtered. The filtrate was purified by column chromatography on silica gel to afford product BN-1 (509 $\mathrm{mg}, 0.87 \mathrm{mmol}, 41 \%$ yield) as a white solid, which was confirmed by X-ray diffraction analysis. ${ }^{1} \mathrm{H}$ NMR $\left(400 \mathrm{MHz}, \mathrm{C}_{6} \mathrm{D}_{6}, 298 \mathrm{~K}\right) \delta 9.01(\mathrm{~d}, J=6.0 \mathrm{~Hz}, 1 \mathrm{H}), 7.24(\mathrm{~d}, J=7.7 \mathrm{~Hz}, 1 \mathrm{H}), 7.13(\mathrm{~d}, J=8.0 \mathrm{~Hz}$, 1H), $7.08(\mathrm{~s}, 4 \mathrm{H}), 6.98(\mathrm{~d}, J=7.4 \mathrm{~Hz}, 1 \mathrm{H}), 6.84(\mathrm{dt}, J=11.8,7.7 \mathrm{~Hz}, 2 \mathrm{H}), 6.76(\mathrm{t}, J=7.4 \mathrm{~Hz}, 1 \mathrm{H}), 6.28$ $(\mathrm{t}, J=6.7 \mathrm{~Hz}, 1 \mathrm{H}), 3.19(\mathrm{~s}, 6 \mathrm{H}), 2.83(\mathrm{p}, J=6.9 \mathrm{~Hz}, 3 \mathrm{H}), 1.28(\mathrm{~d}, J=6.9 \mathrm{~Hz}, 12 \mathrm{H}), 1.06(\mathrm{~s}, 24 \mathrm{H}) .{ }^{13} \mathrm{C}$ NMR $\left(101 \mathrm{MHz}, \mathrm{C}_{6} \mathrm{D}_{6}, 298 \mathrm{~K}\right) \delta 156.30,152.18,148.75,147.01,146.21,145.42,140.05,131.52$, 130.72, 129.92, 127.32, 123.98, 122.67, 121.85, 121.55, 34.14, 32.77, 31.99, 25.37, 24.15. ${ }^{11} \mathrm{~B}$ NMR $\left(128 \mathrm{MHz}, \mathrm{C}_{6} \mathrm{D}_{6}, 298 \mathrm{~K}\right) \delta 5.75$. HR-EIMS (m/z): $[\mathrm{M}]^{+}$calcd. for $\mathrm{C}_{42} \mathrm{H}_{56} \mathrm{BN}, 585.4506$; found 585.4511.

\section{b. Synthesis of BN-Heterocycle BN-2}



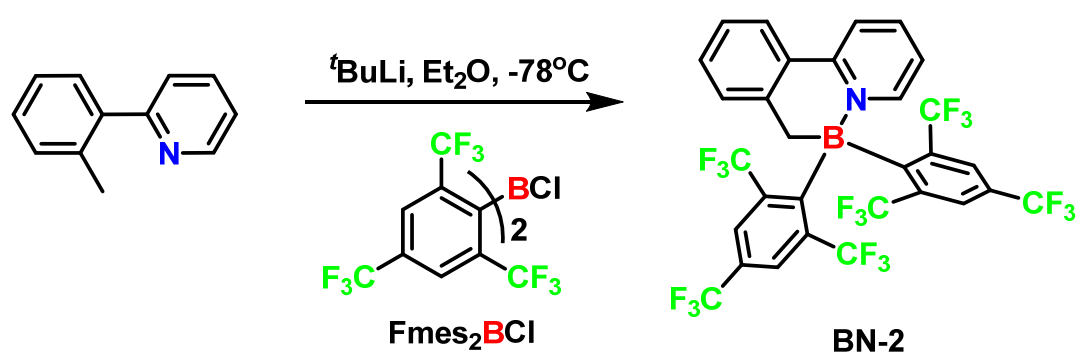

BN-2

Preparation of $\mathrm{Mes}_{2}{ }_{2} \mathrm{BCl}$ : 1,3,5-tris(trifluoromethyl)benzene (1.45 g, $5.12 \mathrm{mmol}$ ) was dissolved in 20 $\mathrm{mL}$ dry $\mathrm{Et}_{2} \mathrm{O}$, the solution was cooled to $-78^{\circ} \mathrm{C}$ in an acetone/dry ice bath for over 30 minutes. n-BuLi $\left(2.05 \mathrm{~mL}, 2.5 \mathrm{M}\right.$ in hexane, $5.12 \mathrm{mmol}$ ) was added dropwise to the reaction mixture at $-78^{\circ} \mathrm{C}$, then allowed to warm up to room temperature. After stirring at room temperature for $3 \mathrm{~h}, \mathrm{BCl}_{3}(2.5 \mathrm{~mL}, 1.0$ $\mathrm{M}$ in hexane, $2.5 \mathrm{mmol}$ ) was added at $-78^{\circ} \mathrm{C}$, and the solution was stirred at $-78^{\circ} \mathrm{C}$ for 30 min and then room temperature for $2 \mathrm{~h}$. This solution of $\mathrm{Mes}_{2}^{\mathrm{F}}{ }_{2} \mathrm{BCl}$ was used directly for next step.

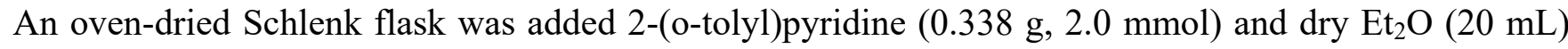
and cooled to $-78^{\circ} \mathrm{C}$ in an acetone/dry ice bath for over 10 minutes. t-BuLi (2.6 mL, $1.7 \mathrm{M}$ in hexane, $4.4 \mathrm{mmol}$ ) was added dropwise to the reaction mixture at $-78^{\circ} \mathrm{C}$. After stirring at $-78^{\circ} \mathrm{C}$ for $1 \mathrm{~h}$, precooled $\mathrm{Mes}{ }_{2}^{\mathrm{F}} \mathrm{BCl}$ solution was added using cannula to the reaction mixture at $-78^{\circ} \mathrm{C}$. The mixture was allowed to warm to room temperature and stirred 24 hours. The mixture was extracted with $\mathrm{CH}_{2} \mathrm{Cl}_{2}$ and water. The filtrate was purified by column chromatography on silica gel to afford product BN-2 (148 $\mathrm{mg}, 0.20 \mathrm{mmol}, 10 \%$ yield) as a white solid, which was confirmed by X-ray diffraction analysis. ${ }^{1} \mathrm{H}$ NMR (499 MHz, C $\left.6 \mathrm{D}_{6}, 298 \mathrm{~K}\right) \delta 8.20(\mathrm{~s}, 2 \mathrm{H}), 8.11(\mathrm{~d}, J=6.3 \mathrm{~Hz}, 1 \mathrm{H}), 7.93(\mathrm{~s}, 1 \mathrm{H}), 7.59(\mathrm{~s}, 1 \mathrm{H}), 6.97-$ $6.77(\mathrm{~m}, 3 \mathrm{H}), 6.66(\mathrm{dt}, J=22.9,7.3 \mathrm{~Hz}, 2 \mathrm{H}), 6.44(\mathrm{~d}, J=7.4 \mathrm{~Hz}, 1 \mathrm{H}), 6.31-6.19(\mathrm{~m}, 1 \mathrm{H}), 3.09(\mathrm{~s}, 1 \mathrm{H})$, $2.08(\mathrm{~s}, 1 \mathrm{H}) .{ }^{1} \mathrm{H}$ NMR $\left(499 \mathrm{MHz}, \mathrm{C}_{6} \mathrm{D}_{6}, 278 \mathrm{~K}\right) \delta 8.20(\mathrm{~d}, J=10.0 \mathrm{~Hz}, 2 \mathrm{H}), 8.06(\mathrm{~d}, J=6.3 \mathrm{~Hz}, 1 \mathrm{H})$, $7.94(\mathrm{~s}, 1 \mathrm{H}), 7.60(\mathrm{~s}, 1 \mathrm{H}), 6.90(\mathrm{~d}, J=7.7 \mathrm{~Hz}, 1 \mathrm{H}), 6.85-6.72(\mathrm{~m}, 2 \mathrm{H}), 6.67(\mathrm{t}, J=7.6 \mathrm{~Hz}, 1 \mathrm{H}), 6.62(\mathrm{t}$, $J=7.5 \mathrm{~Hz}, 1 \mathrm{H}), 6.43(\mathrm{~d}, J=7.5 \mathrm{~Hz}, 1 \mathrm{H}), 6.20(\mathrm{t}, J=6.5 \mathrm{~Hz}, 1 \mathrm{H}), 3.11(\mathrm{~d}, J=15.6 \mathrm{~Hz}, 1 \mathrm{H}), 2.09(\mathrm{~d}, J=$ $15.6 \mathrm{~Hz}, 1 \mathrm{H}) .{ }^{1} \mathrm{H}$ NMR $\left(400 \mathrm{MHz}, \mathrm{CDCl}_{3}, 298 \mathrm{~K}\right) \delta 8.60(\mathrm{~d}, J=6.1 \mathrm{~Hz}, 1 \mathrm{H}), 8.32-8.00(\mathrm{~m}, 3 \mathrm{H}), 7.97$ $(\mathrm{dd}, J=8.2,1.4 \mathrm{~Hz}, 1 \mathrm{H}), 7.76(\mathrm{~s}, 1 \mathrm{H}), 7.56-7.31(\mathrm{~m}, 3 \mathrm{H}), 7.07(\mathrm{td}, J=7.6,1.3 \mathrm{~Hz}, 1 \mathrm{H}), 6.99(\mathrm{td}, J=$ $7.5,1.4 \mathrm{~Hz}, 1 \mathrm{H}), 6.58(\mathrm{dd}, J=7.5,1.3 \mathrm{~Hz}, 1 \mathrm{H}), 2.98(\mathrm{~s}, 1 \mathrm{H}), 1.91(\mathrm{~s}, 1 \mathrm{H}) .{ }^{13} \mathrm{C} \mathrm{NMR}\left(101 \mathrm{MHz}, \mathrm{CDCl}_{3}\right.$, $298 \mathrm{~K}) \delta 155.67,150.21,142.73,142.61,131.52,130.06,128.38,128.11,127.38,127.01,125.35$, 123.87, 123.00(-CF3, q, J $=274 \mathrm{~Hz}, 124.33,121.62), 122.22,30.79 .{ }^{13} \mathrm{C} \mathrm{NMR}\left(126 \mathrm{MHz}, \mathrm{CDCl}_{3}\right.$, $278 \mathrm{~K}) \delta 155.50,150.23,142.73,142.67,138.47-135.12\left(-\mathrm{C}_{-} \mathrm{CF}_{3}\right), 131.52,130.02,130.00-121.00(-$ $\left.\mathrm{C}-\mathrm{CF}_{3},-\mathrm{CF}_{3}\right), 128.34,128.04,127.42,127.21,126.90,125.39,123.93,122.33,30.71 .{ }^{19} \mathrm{~F}$ NMR $(376$ $\left.\mathrm{MHz}, \mathrm{C}_{6} \mathrm{D}_{6}, 298 \mathrm{~K}\right) \delta-51.58,-52.15,-52.87--53.94(\mathrm{~m}),-63.96,-64.39 .{ }^{11} \mathrm{~B}$ NMR $\left(128 \mathrm{MHz}, \mathrm{C}_{6} \mathrm{D}_{6}\right.$, $298 \mathrm{~K}) \delta-0.03 .{ }^{19} \mathrm{~F}$ NMR $\left(376 \mathrm{MHz}, \mathrm{C}_{6} \mathrm{D}_{6}, 298 \mathrm{~K}\right) \delta-51.58,-52.15,-53.45,-53.51,-63.96,-64.39$. HREIMS (m/z): [M] $]^{+}$calcd. for $\mathrm{C}_{30} \mathrm{H}_{14} \mathrm{BNF}_{18}$ : 741.0932; found: 741.0932 . 


\section{c. Synthesis of BN-Heterocycle BN-3}
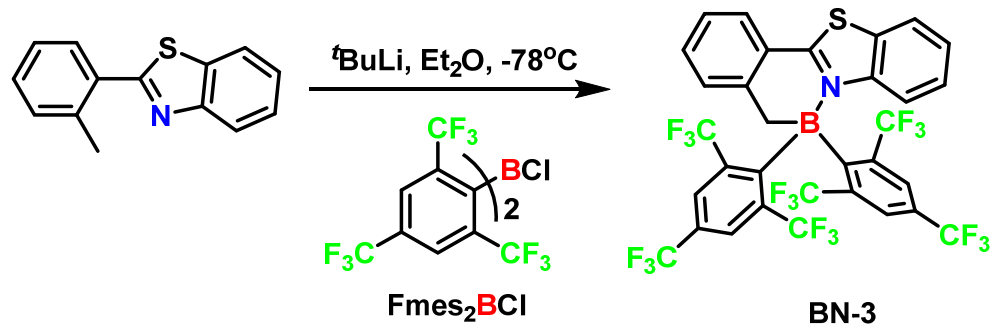

To an oven-dried Schlenk flask was added 2-(o-tolyl)benzo[d]thiazole (340 $\mathrm{mg}, 1.51 \mathrm{mmol})$ and dry $\mathrm{Et}_{2} \mathrm{O}(20 \mathrm{~mL})$ and cooled to $-78^{\circ} \mathrm{C}$ in an acetone/dry ice bath for over 10 minutes. t-BuLi $(2.0 \mathrm{~mL}, 1.7$ $\mathrm{M}$ in hexane, $3.24 \mathrm{mmol}$ ) was added dropwise to the reaction mixture at $-78^{\circ} \mathrm{C}$. After stirring at $-78^{\circ} \mathrm{C}$ for $1 \mathrm{~h}$, pre-cooled $\mathrm{Mes}_{2}{ }_{2} \mathrm{BCl}$ (1.5 eq) solution was added using cannula to the reaction mixture at $78^{\circ} \mathrm{C}$. The mixture was allowed to warm to room temperature and stirred 24 hours. The mixture was extracted with $\mathrm{CH}_{2} \mathrm{Cl}_{2}$ and water. The filtrate was purified by column chromatography on silica gel to afford product BN-3 (217 mg, $0.27 \mathrm{mmol}, 18 \%$ yield) as a white solid. ${ }^{1} \mathrm{H}$ NMR (400 MHz, $\mathrm{C}_{6} \mathrm{D}_{6}, 298 \mathrm{~K}$ ) $\delta 8.32(\mathrm{~s}, 1 \mathrm{H}), 8.17(\mathrm{~s}, 1 \mathrm{H}), 7.92(\mathrm{~s}, 1 \mathrm{H}), 7.67(\mathrm{~d}, J=8.7 \mathrm{~Hz}, 1 \mathrm{H}), 7.59(\mathrm{~s}, 1 \mathrm{H}), 7.03(\mathrm{~d}, J=7.5 \mathrm{~Hz}, 1 \mathrm{H})$, 6.97 (dd, $J=8.0,1.4 \mathrm{~Hz}, 1 \mathrm{H}), 6.72-6.65(\mathrm{~m}, 1 \mathrm{H}), 6.63(\mathrm{ddt}, J=6.4,3.0,1.4 \mathrm{~Hz}, 2 \mathrm{H}), 6.60-6.49(\mathrm{~m}$, $2 \mathrm{H}), 3.11(\mathrm{~s}, 1 \mathrm{H}), 2.31-2.12(\mathrm{~m}, 1 \mathrm{H}) .{ }^{13} \mathrm{C}$ NMR $\left(126 \mathrm{MHz}, \mathrm{CD}_{2} \mathrm{Cl}_{2}, 298 \mathrm{~K}\right) \delta 173.96,149.25,143.45$, 138.06, 133.51, 129.69, 128.75, 128.52, 127.92, 127.70, 127.56, 127.53, 127.27, 126.89, 126.78, 126.71, $126.30,125.64,125.12,124.22,124.04,122.91,122.71,121.00,120.82,109.99,32.13 .{ }^{19} \mathrm{~F}$ NMR (376 $\left.\mathrm{MHz}, \mathrm{C}_{6} \mathrm{D}_{6}, 298 \mathrm{~K}\right) \delta-51.32,-51.96,-52.30(\mathrm{t}, J=19.5 \mathrm{~Hz}),-53.02--54.31(\mathrm{~m}),-63.98,-64.37 .{ }^{11} \mathrm{~B}$ NMR $\left(128 \mathrm{MHz}, \mathrm{C}_{6} \mathrm{D}_{6}, 298 \mathrm{~K}\right) \delta$-1.52. HR-EIMS (m/z): $[\mathrm{M}]^{+}$calcd. for $\mathrm{C}_{32} \mathrm{H}_{14} \mathrm{BNF}_{18} \mathrm{~S}: 797.0653$; found: 797.0645 .

\section{d. Synthesis of BN-Heterocycle BN-5}
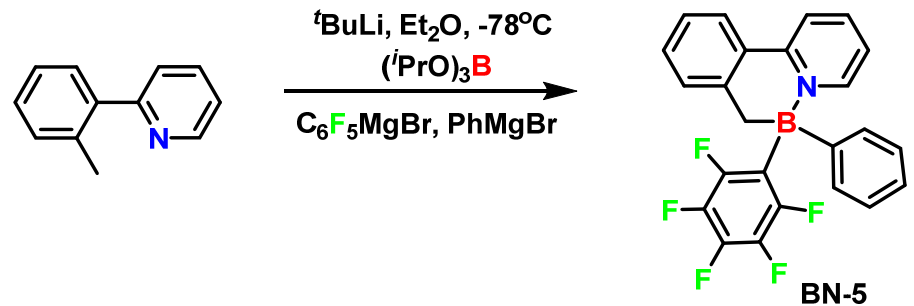

t-BuLi (1.68 $\mathrm{mL}, 1.7 \mathrm{M}$ in hexane, $2.86 \mathrm{mmol})$ was added to a solution of 2-(o-tolyl)pyridine (220 $\mathrm{mg}$, $1.30 \mathrm{mmol})$ in $\mathrm{Et}_{2} \mathrm{O}(15 \mathrm{~mL})$ at $-78^{\circ} \mathrm{C}$. After stirring at this temperature for $1 \mathrm{~h}, \mathrm{~B}\left(\mathrm{O}^{i} \mathrm{Pr}\right)_{3}(360 \mathrm{ul}, 1.56$ mmol) was added. The reaction solution was stirred for $1 \mathrm{~h}$ at the same temperature, after which pentafluorophenylmagnesium bromide $\left(\mathrm{C}_{6} \mathrm{~F}_{5} \mathrm{MgBr}\right)(2.9 \mathrm{ml}, 0.5 \mathrm{M}$ in diethyl ether, $1.43 \mathrm{mmol})$ was added at $-78^{\circ} \mathrm{C}$. Next, phenylmagnesium bromide $(0.43 \mathrm{ml}, 3 \mathrm{M}$ in diethyl ether, $1.30 \mathrm{mmol})$ was added at $-78{ }^{\circ} \mathrm{C}$, and the mixture was warmed slowly to room temperature and stirred overnight. The mixture was extracted with $\mathrm{CH}_{2} \mathrm{Cl}_{2}$ and water. The filtrate was purified by column chromatography on silica gel to afford product $\mathbf{B N}-5$ ( $233 \mathrm{mg}, 0.55 \mathrm{mmol}, 42 \%$ yield) as a white solid, which was confirmed by X-ray diffraction analysis. ${ }^{1} \mathrm{H}$ NMR $\left(400 \mathrm{MHz}, \mathrm{C}_{6} \mathrm{D}_{6}, 298 \mathrm{~K}\right) \delta 8.05(\mathrm{~d}, J=6.1 \mathrm{~Hz}, 1 \mathrm{H}), 7.50-7.43(\mathrm{~m}, 2 \mathrm{H})$, $7.43-7.37(\mathrm{~m}, 2 \mathrm{H}), 7.34-7.29(\mathrm{~m}, 1 \mathrm{H}), 7.20(\mathrm{~d}, J=7.6 \mathrm{~Hz}, 1 \mathrm{H}), 7.10(\mathrm{dd}, J=7.8,1.2 \mathrm{~Hz}, 1 \mathrm{H}), 7.00$ (td, $J=7.5,1.3 \mathrm{~Hz}, 1 \mathrm{H}), 6.98-6.93(\mathrm{~m}, 1 \mathrm{H}), 6.83(\mathrm{tt}, J=7.6,1.2 \mathrm{~Hz}, 1 \mathrm{H}), 6.75(\mathrm{td}, J=7.9,1.7 \mathrm{~Hz}$, 
$1 \mathrm{H}), 6.05(\mathrm{ddd}, J=7.5,6.0,1.4 \mathrm{~Hz}, 1 \mathrm{H}), 2.84(\mathrm{dt}, J=15.3,2.2 \mathrm{~Hz}, 1 \mathrm{H}), 2.65(\mathrm{~d}, J=15.4 \mathrm{~Hz}, 1 \mathrm{H}) .{ }^{13} \mathrm{C}$ NMR $\left(101 \mathrm{MHz}, \mathrm{CD}_{2} \mathrm{Cl}_{2}, 298 \mathrm{~K}\right) \delta 154.36,146.82,145.51,141.59,133.70,131.44,129.89,129.57$, $127.91,126.64,125.27,123.31,122.69,26.81$. (Due to the coupling of fluoride to carbon, Carbon atoms connecting to fluoride did not show up) ${ }^{11} \mathrm{~B}$ NMR $\left(128 \mathrm{MHz}, \mathrm{C}_{6} \mathrm{D}_{6}, 298 \mathrm{~K}, \delta\right.$, ppm): $-0.43 .{ }^{19} \mathrm{~F}$ NMR (376 MHz, $\mathrm{C}_{6} \mathrm{D}_{6}, 298 \mathrm{~K}, \delta$, ppm): -135.16 (d, $\left.J=24.3 \mathrm{~Hz}\right),-159.70$ (d, $J=44.6 \mathrm{~Hz}$ ), -164.54 - -166.59 (m). HR-EIMS (m/z): [M] ${ }^{+}$calcd. for $\mathrm{C}_{24} \mathrm{H}_{15} \mathrm{BNF}_{5}$ : 423.1218 ; found: 423.1227.

\section{e. Synthesis of BN-Heterocycle BN-6}
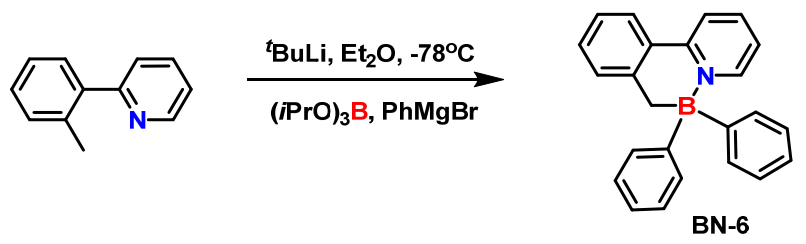

t-BuLi (1.3 mL, 1.7 M in hexane, $2.2 \mathrm{mmol})$ was added to a solution of 2-(o-tolyl)pyridine (169 $\mathrm{mg}, 1$ $\mathrm{mmol})$ in $\mathrm{Et}_{2} \mathrm{O}(15 \mathrm{~mL})$ at $-78^{\circ} \mathrm{C}$. After stirring at this temperature for $1 \mathrm{~h}, \mathrm{~B}\left(\mathrm{O}^{i} \mathrm{Pr}\right)_{3}(226 \mathrm{mg}, 1.2 \mathrm{mmol})$ was added. The mixture was stirred for another hour at $-78{ }^{\circ} \mathrm{C}$, after which phenylmagnesium bromide $\left(1.0 \mathrm{ml}, 3 \mathrm{M}\right.$ in diethyl ether, $3 \mathrm{mmol}$ ) was added at $-78{ }^{\circ} \mathrm{C}$, then allowed to warm slowly to room temperature and stirred overnight. The mixture was extracted with $\mathrm{CH}_{2} \mathrm{Cl}_{2}$ and water. The filtrate was purified by column chromatography on silica gel to afford product BN-6 (270 mg, $0.81 \mathrm{mmol}, 81 \%$ yield) as a white solid, which was confirmed by X-ray diffraction analysis. ${ }^{1} \mathrm{H} \mathrm{NMR} \mathrm{(400} \mathrm{MHz}, \mathrm{CDCl}_{3}$, $298 \mathrm{~K}) \delta 8.35(\mathrm{~d}, J=5.8 \mathrm{~Hz}, 1 \mathrm{H}), 8.02(\mathrm{~d}, J=2.7 \mathrm{~Hz}, 2 \mathrm{H}), 7.59(\mathrm{~d}, J=7.8 \mathrm{~Hz}, 1 \mathrm{H}), 7.38-7.25(\mathrm{~m}, 3 \mathrm{H})$, $7.25-7.17(\mathrm{~m}, 4 \mathrm{H}), 7.17-7.05(\mathrm{~m}, 7 \mathrm{H}), 2.64(\mathrm{~s}, 2 \mathrm{H}) .{ }^{13} \mathrm{C} \mathrm{NMR}\left(101 \mathrm{MHz}, \mathrm{CDCl}_{3}, 298 \mathrm{~K}\right) \delta 154.50$, $146.77,146.31,140.42,133.46,131.40,130.63,129.84,127.13,126.71,125.15,124.90,122.92,122.36$, 27.90. ${ }^{11} \mathrm{~B}$ NMR $\left(128 \mathrm{MHz}, \mathrm{CDCl}_{3}, 298 \mathrm{~K}\right) \delta 0.06$. HR-EIMS $(\mathrm{m} / \mathrm{z}):[\mathrm{M}]^{+}$calcd. for $\mathrm{C}_{24} \mathrm{H}_{20} \mathrm{BN}$, 333.1689 ; found 333.1698 .

\section{Investigation of the Photo-reactivity of BN-heterocycles}

General photolysis reaction procedure: ${ }^{1} \mathrm{H}$ NMR scale photoelimination reactions were carried out under $\mathrm{N}_{2}$ using $\mathrm{C}_{6} \mathrm{D}_{6}$ as the solvent, quartz J-Young NMR tubes as the reaction vessels, and a Rayonet Photochemical Reactor as the light source. $\Phi_{\mathrm{PE}}$ of $\mathbf{B N}-1$ was determined using competitive photolysis experiments. ${ }^{4}$

3.1 Photoelimination of BN-Heterocycle BN-1: Compound BN-1 ( $20 \mathrm{mg})$ was dissolved in $\mathrm{C}_{6} \mathrm{D}_{6}(0.5$ $\mathrm{mL}$ ) in a quartz J-Young NMR tube and sealed under nitrogen. The NMR tube was then irradiated with $300 \mathrm{~nm}$ UV light and the reaction progress was monitored by ${ }^{1} \mathrm{H},{ }^{11} \mathrm{~B}$ and ${ }^{19} \mathrm{~F} \mathrm{NMR}$ spectra until it reached completion. The product $\mathbf{B N}-$ phen-1 can be isolated as bright yellow crystals from the slow evaporation of the benzene solution. Its structure was confirmed by X-ray diffraction analysis.

BN-phen-1: ${ }^{1} \mathrm{H}$ NMR $\left(499 \mathrm{MHz}, \mathrm{C}_{6} \mathrm{D}_{6}\right) \delta 8.43(\mathrm{~d}, J=6.9 \mathrm{~Hz}, 1 \mathrm{H}), 8.10(\mathrm{~d}, J=8.8 \mathrm{~Hz}, 1 \mathrm{H}), 8.04(\mathrm{~d}, J=$ $9.1 \mathrm{~Hz}, 1 \mathrm{H}), 7.70(\mathrm{~d}, J=8.4 \mathrm{~Hz}, 1 \mathrm{H}), 7.37(\mathrm{~s}, 3 \mathrm{H}), 7.28(\mathrm{t}, J=7.5 \mathrm{~Hz}, 1 \mathrm{H}), 7.01(\mathrm{~d}, J=7.5 \mathrm{~Hz}, 1 \mathrm{H})$, $6.71(\mathrm{t}, J=7.9 \mathrm{~Hz}, 1 \mathrm{H}), 6.13(\mathrm{t}, J=6.8 \mathrm{~Hz}, 1 \mathrm{H}), 2.99(\mathrm{p}, J=7.0 \mathrm{~Hz}, 1 \mathrm{H}), 2.89(\mathrm{q}, J=6.8 \mathrm{~Hz}, 2 \mathrm{H}), 1.39$ $(\mathrm{d}, J=6.9 \mathrm{~Hz}, 6 \mathrm{H}), 1.27(\mathrm{~d}, J=6.8 \mathrm{~Hz}, 6 \mathrm{H}), 1.15(\mathrm{~d}, J=6.9 \mathrm{~Hz}, 6 \mathrm{H}) .{ }^{13} \mathrm{C} \mathrm{NMR}\left(101 \mathrm{MHz}, \mathrm{C}_{6} \mathrm{D}_{6}, \delta\right.$, ppm): 151.82, 149.01, 148.87, 144.84, 142.46, 137.58, 128.88, 128.74, 128.34, 124.71, 120.57, 119.83, 
118.97, 115.46, 35.02, 34.92, 25.07, 24.42, 24.38. ${ }^{11} \mathrm{~B}$ NMR (128 MHz, $\left.\mathrm{C}_{6} \mathrm{D}_{6}\right) \delta 36.12$. HR-ESIMS (m/z): $[\mathrm{M}+1]^{+}$calcd. for $\mathrm{C}_{27} \mathrm{H}_{33} \mathrm{NB}$ : 382.27006; found: 382.26844 .
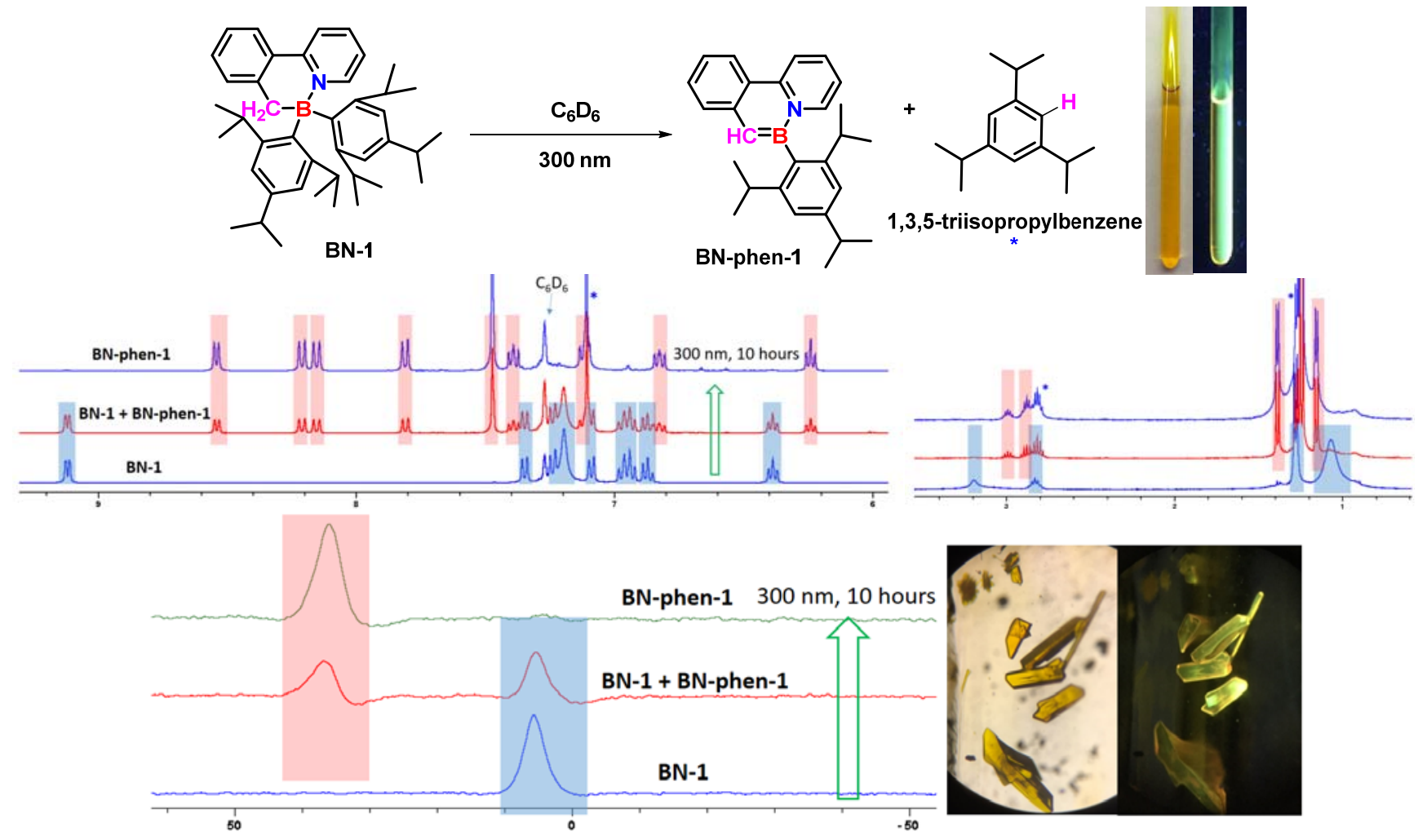

Figure S3A. ${ }^{1} \mathrm{H}$ (top spectrum) and ${ }^{11} \mathrm{~B}$ (bottom spectrum) NMR spectra showing the clean conversion of BN-1 (blue highlight) to BN-phen-1 (red highlight) in C6D6 at room temperature and $300 \mathrm{~nm}$ irradiation. Photos of BN-phen-1 crystals under microscope (bottom right).
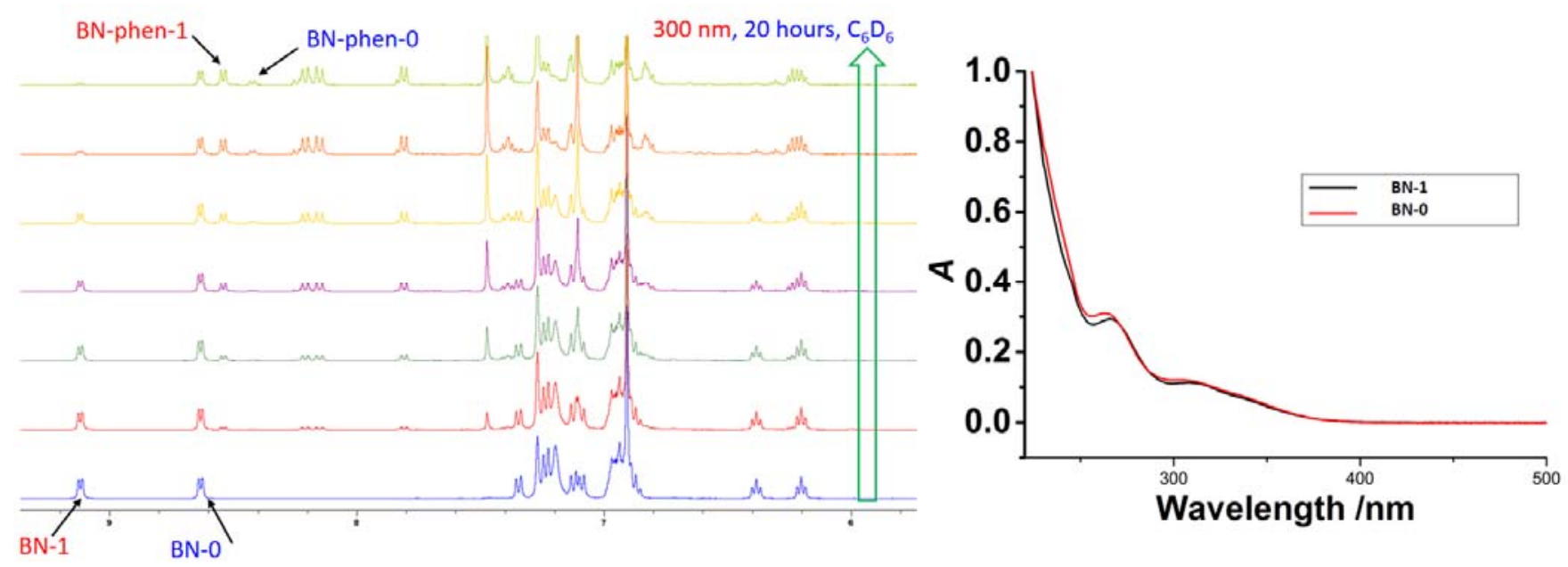

Figure S3.1B. NMR competition experiment. Left: ${ }^{1} \mathrm{H}$ NMR spectra of $\mathbf{B N}-\mathbf{1}$ and $\mathbf{B N - 0}$ showing the relative conversion rate to BN-phen-1 and BN-phen-0 after irradiation with $U V(300 \mathrm{~nm})$ in $\mathrm{C}_{6} \mathrm{D}_{6}$ under $\mathrm{N}_{2}$. Right: UV/Vis spectra of BN-1 and $\mathbf{B N - 0}$ in THF $\left(1.0 \times 10^{-5} \mathrm{M}\right)$ showing their similar absorption profile. The photoelimination quantum yield of $\mathbf{B N - 0}$ is about $4.4 \%$ and that of $\mathbf{B N}-1$ was determined to be $\sim 0.25$. 
3.2 Photoisomerization of BN-Heterocycle BN-2: Compound BN-2 $(\sim 20 \mathrm{mg})$ was dissolved in $\mathrm{C}_{6} \mathrm{D}_{6}$ $(0.5 \mathrm{~mL})$ in a quartz J-Young NMR tube and sealed under nitrogen. The NMR tube was then irradiated with $300 \mathrm{~nm}$ UV light and the reaction progress was monitored by ${ }^{1} \mathrm{H}$ and ${ }^{11} \mathrm{~B}$ NMR spectra until it reached completion. The product of BN2a can be isolated quantitatively as dark orange brown crystals from a benzene/hexane solution. The structure of BN-2a was confirmed by X-ray diffraction analysis.

BN-2a: ${ }^{1} \mathrm{H}$ NMR $\left(400 \mathrm{MHz}, \mathrm{C}_{6} \mathrm{D}_{6}\right) \delta 8.17(\mathrm{~d}, J=1.8 \mathrm{~Hz}, 1 \mathrm{H}), 8.02(\mathrm{~d}, J=6.4$ $\mathrm{Hz}, 1 \mathrm{H}), 7.88-7.78(\mathrm{~m}, 1 \mathrm{H}), 7.09-7.04(\mathrm{~m}, 1 \mathrm{H}), 7.05-6.92(\mathrm{~m}, 2 \mathrm{H}), 6.84$ $(\mathrm{dd}, J=7.5,1.3 \mathrm{~Hz}, 1 \mathrm{H}), 6.50(\mathrm{td}, J=7.7,1.4 \mathrm{~Hz}, 1 \mathrm{H}), 6.42(\mathrm{dd}, J=8.0,1.6$ $\mathrm{Hz}, 1 \mathrm{H}), 6.11(\mathrm{~s}, 1 \mathrm{H}), 5.99(\mathrm{~s}, 1 \mathrm{H}), 5.93(\mathrm{ddd}, J=7.8,6.5,1.7 \mathrm{~Hz}, 1 \mathrm{H}), 2.60-$ $2.35(\mathrm{~m}, 2 \mathrm{H}) .{ }^{13} \mathrm{C}$ NMR $\left(101 \mathrm{MHz}, \mathrm{C}_{6} \mathrm{D}_{6}\right) \delta 160.14(\mathrm{C} 5), 147.45(\mathrm{~d}, \mathrm{~J}=2.9 \mathrm{~Hz}$, C1), 140.76 (C3), 138.11 (C11), 138.11 (q, J = $47 \mathrm{~Hz}), 136.06$ (C6), 135.19 (q,

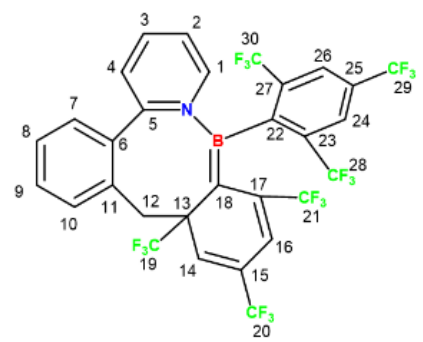
$\mathrm{J}=30 \mathrm{~Hz}), 131.52(\mathrm{C} 9), 131.28(\mathrm{q}, \mathrm{J}=32 \mathrm{~Hz}), 130.95(\mathrm{q}, \mathrm{J}=35 \mathrm{~Hz}), 129.26(\mathrm{C} 7), 128.95(\mathrm{C} 4), 128.71$ (C10), 126.63(C8), 126.35 (br, C24 or C26), 125.94 (br, C14), 125.72 (br, C24 or C26), 123.03 (C2), 123.80 (q, J $=245 \mathrm{~Hz}, \mathrm{C} 19$ (-CF $\left.\left.{ }_{3}\right), 127.80,125.35,122.15,119.89\right), 123.39(\mathrm{q}, \mathrm{J}=297 \mathrm{~Hz}, \mathrm{C} 28$ or C30($\left.\left.\mathrm{CF}_{3}\right), 124.87,121.90\right), 123.25\left(\mathrm{q}, \mathrm{J}=271 \mathrm{~Hz}, \mathrm{C} 21\left(-\mathrm{CF}_{3}\right), 126.45,124.60,121.89,118.60\right), 123.15(\mathrm{q}, \mathrm{J}=$ $\left.275 \mathrm{~Hz}, \mathrm{C} 20\left(-\mathrm{CF}_{3}\right), 127.10,124.36,121.64,118.94\right), 123.00\left(\mathrm{q}, \mathrm{J}=272 \mathrm{~Hz}, \mathrm{C} 29\left(-\mathrm{CF}_{3}\right)\right.$, 124.36,121.64),128-120, 106.18 (q, J = $9 \mathrm{~Hz}, \mathrm{C} 16), 56.34$ (q, J = $25 \mathrm{~Hz}, \mathrm{C} 13), 43.53$ (C12). ${ }^{19} \mathrm{~F}$ NMR $\left(376 \mathrm{MHz}, \mathrm{C}_{6} \mathrm{D}_{6}\right) \delta-56.70(\mathrm{q}, J=16.4,15.9 \mathrm{~Hz}),-58.06,-58.87(\mathrm{~d}, J=11.7 \mathrm{~Hz}),-64.08,-68.98,-79.70$. ${ }^{11} \mathrm{~B}$ NMR (128 MHz, $\left.\mathrm{C}_{6} \mathrm{D}_{6}, \delta\right): 35.14$. HR-ESIMS (m/z): $[\mathrm{M}+1]^{+}$calcd. for $\mathrm{C}_{30} \mathrm{H}_{15} \mathrm{NBF}_{18}, 742.10046$; found 742.10156 .
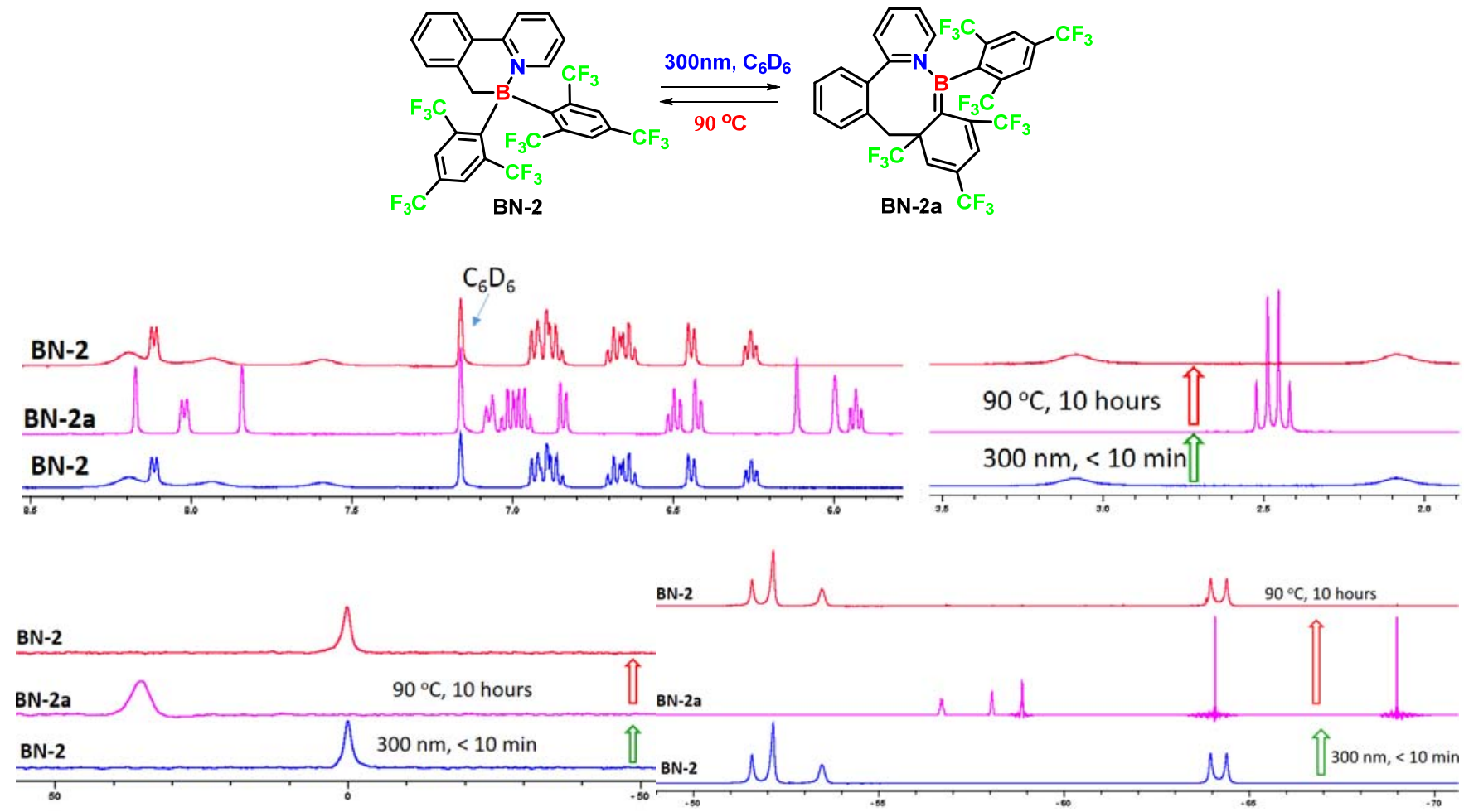

Figure S3.2A. ${ }^{1} \mathrm{H}$ (top), ${ }^{11} \mathrm{~B}$ (bottom, left), ${ }^{19} \mathrm{~F}$ (bottom, right) NMR spectra showing the clean and reversible conversion of $\mathbf{B N}-2$ to $\mathbf{B N}-2 \mathrm{a}$ in C6D6. 


\subsection{The Fatigue Resistance of BN-2}

Compound BN-2 $(\sim 10 \mathrm{mg})$ was dissolved in $\mathrm{C}_{6} \mathrm{D}_{6}(0.5 \mathrm{~mL})$ in a quartz J-Young NMR tube and sealed under nitrogen. The integration ratio between the peak at $6.44 \mathrm{ppm}$ and the peak of $\mathrm{C}_{6} \mathrm{D}_{6}$ was recorded as cycle 0. The NMR tube was then irradiated with $300 \mathrm{~nm}$ UV light, after BN-2 completely converted into BN-2a (cycle 0.5), the integration ratio between the peak at $5.99 \mathrm{ppm}$ of $\mathbf{B N}-\mathbf{2 a}$ and the peak of $\mathrm{C}_{6} \mathrm{D}_{6}$ was recorded. Next, BN-2a was converted back to $\mathbf{B N}-2$ at $90{ }^{\circ} \mathrm{C}$ (cycle 1) and the integration ratio of the peak at $6.44 \mathrm{pm}$ was recorded again. The cycling was repeated until cycle 13.5 was reached.
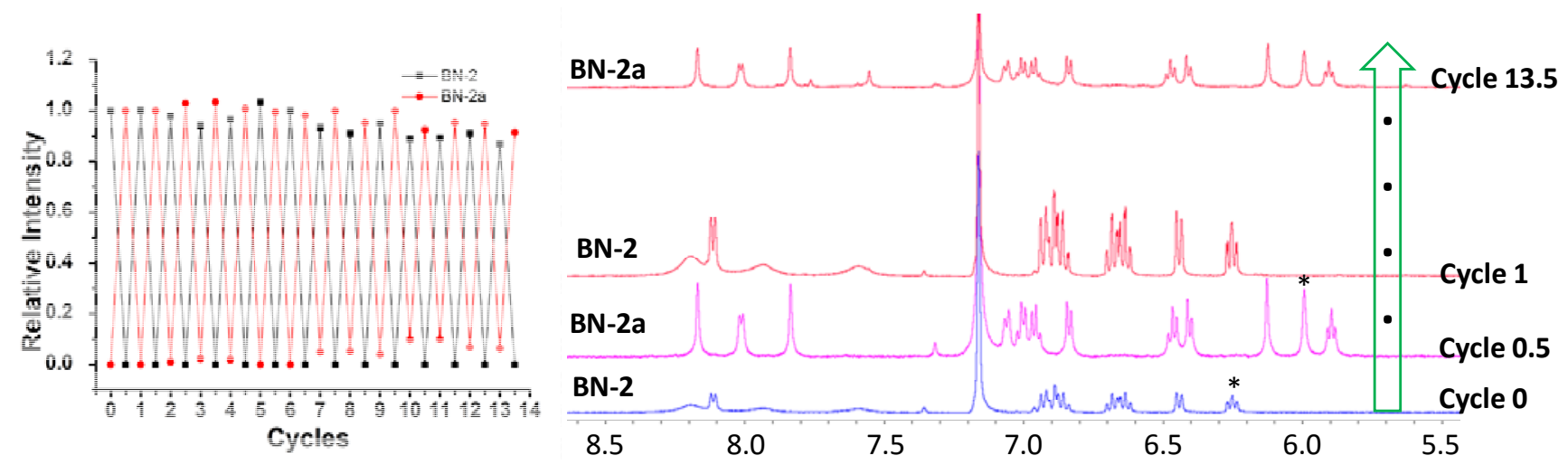

Figure S3.3. Fatigue Resistance of photoisomerization of BN-2. X axis: the times of cycles. Y axis: relative integrated intensity of $\mathbf{B N}-2$ and $\mathbf{B N}-2$ a compared to cycle 0 . The peaks chosen for integration relative to the solvent peak $\left(\mathrm{C}_{6} \mathrm{D}_{6}\right)$ are marked by $*$. ${ }^{1} \mathrm{H}$ NMR data show there is no obvious decomposition until after 13 cycles.

\subsection{Activation Energy of Thermal Transformation from BN-2a to BN-2}

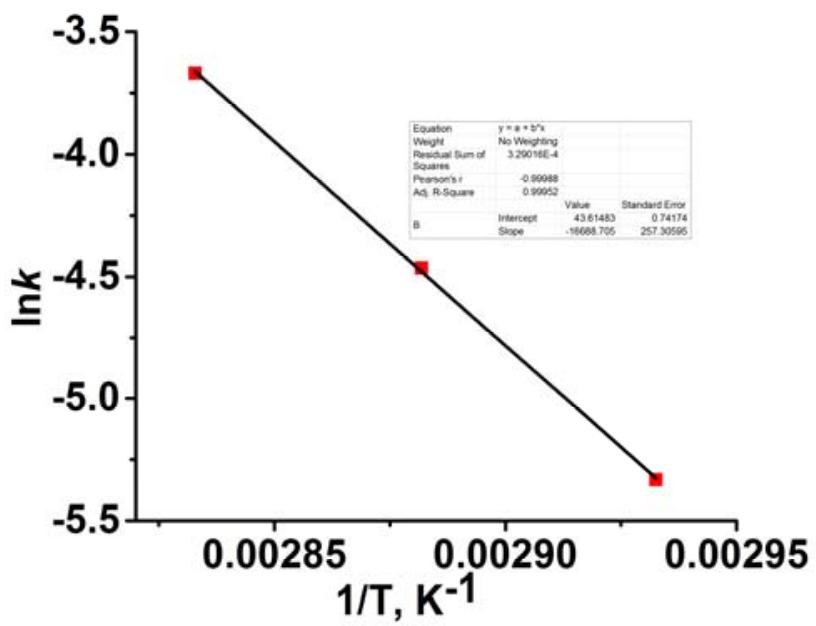

Figure S3.4. Temperature dependence of the rate constant $k$ for thermal reaction $\mathbf{B N}-\mathbf{2 a} \rightarrow \mathbf{B N}-2$. 
3.5 Photoisomerization of BN-Heterocycle BN-3: Compound BN-3 $(\sim 20 \mathrm{mg})$ was dissolved in $\mathrm{C}_{6} \mathrm{D}_{6}$ $(0.5 \mathrm{~mL})$ in a quartz J-Young NMR tube and sealed under nitrogen. The NMR tube was then irradiated with $300 \mathrm{~nm}$ UV light and the reaction progress was monitored by ${ }^{1} \mathrm{H}$ and ${ }^{11} \mathrm{~B}$ NMR spectra until it reached completion. The product BN-3a can be isolated by crystallization from solution of benzene/hexanes as dark brown microcrystalline solid.

BN-3a: ${ }^{1} \mathrm{H}$ NMR $\left(499 \mathrm{MHz}, \mathrm{C}_{6} \mathrm{D}_{6}\right) \delta 8.23(\mathrm{~s}, 1 \mathrm{H}), 7.72(\mathrm{~d}, J=8.6 \mathrm{~Hz}, 1 \mathrm{H}), 7.69$ (s, $1 \mathrm{H}), 7.28(\mathrm{~d}, J=7.7 \mathrm{~Hz}, 1 \mathrm{H}), 6.97(\mathrm{t}, J=7.6 \mathrm{~Hz}, 1 \mathrm{H}), 6.87(\mathrm{q}, J=7.8 \mathrm{~Hz}$, $2 \mathrm{H}), 6.75(\mathrm{~d}, J=7.8 \mathrm{~Hz}, 1 \mathrm{H}), 6.62(\mathrm{dt}, J=18.6,7.2 \mathrm{~Hz}, 2 \mathrm{H}), 6.07(\mathrm{~s}, 1 \mathrm{H}), 5.96$ (s, $1 \mathrm{H}), 2.64(\mathrm{~d}, J=14.1 \mathrm{~Hz}, 1 \mathrm{H}), 2.35(\mathrm{~d}, J=14.0 \mathrm{~Hz}, 1 \mathrm{H}) .{ }^{13} \mathrm{C}$ NMR $(126$ $\left.\mathrm{MHz}, \mathrm{C}_{6} \mathrm{D}_{6}\right) \delta 178.44(\mathrm{C} 7), 146.26$ (br, $\mathrm{C} 20$ or C24), $145.84(\mathrm{C} 5), 139.78(\mathrm{q}, \mathrm{J}=$ $27 \mathrm{~Hz}), 139.26(\mathrm{C} 8), 138.85(\mathrm{C} 17, \mathrm{q}, \mathrm{J}=25 \mathrm{~Hz}), 134.74(\mathrm{q}, \mathrm{J}=27 \mathrm{~Hz}), 133.26$

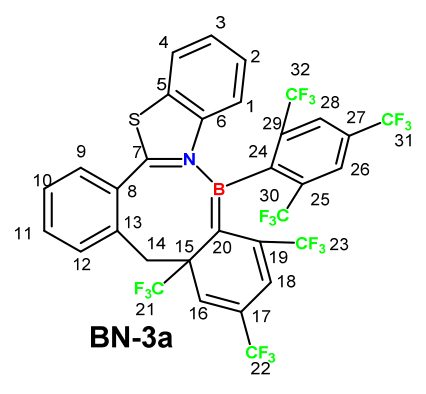
(C12), 130.92 (C6), 130.86 (q, J = $31 \mathrm{~Hz}), 130.77(\mathrm{C} 19$, q, J = $31 \mathrm{~Hz}), 130.14$ (C13), 129.46(C9), 129.09 (C11), 128.41 (C3), 127.13 (C2), 126.70 (C10), 126.65 (br, C26 or C28), 125.93 (br, C26 or C28), 124.21 (br, C16), 123.00 (C22, q, J = $272 \mathrm{~Hz}), 121.88$ (C4), 120.57 (C1, q, J = 4 Hz), 131-119 (C21, C23, C25, C27, C29-C32), 105.10 (C18, q, J = 8 Hz), 100.88 (br, C20 or C24), 56.44 (C15, q, J = $24 \mathrm{~Hz}), 45.80(\mathrm{C} 14) .{ }^{19} \mathrm{~F}$ NMR $\left(376 \mathrm{MHz}, \mathrm{C}_{6} \mathrm{D}_{6}, \delta, \mathrm{ppm}\right):-54.44$ (q, $\left.J=13.9 \mathrm{~Hz}\right),-58.05$ (d, $J=11.5$ $\mathrm{Hz}),-59.27$ (q, $J=10.3 \mathrm{~Hz}),-64.20,-69.09,-79.39$ (broad). ${ }^{11} \mathrm{~B}$ NMR $\left(128 \mathrm{MHz}, \mathrm{C}_{6} \mathrm{D}_{6}, \delta, \quad\right.$ ppm): 33.95. HR-ESIMS (m/z): [M+1] calcd. for $\mathrm{C}_{32} \mathrm{H}_{15} \mathrm{NBF}_{18} \mathrm{~S}$ : 798.07253; found: 798.07440.

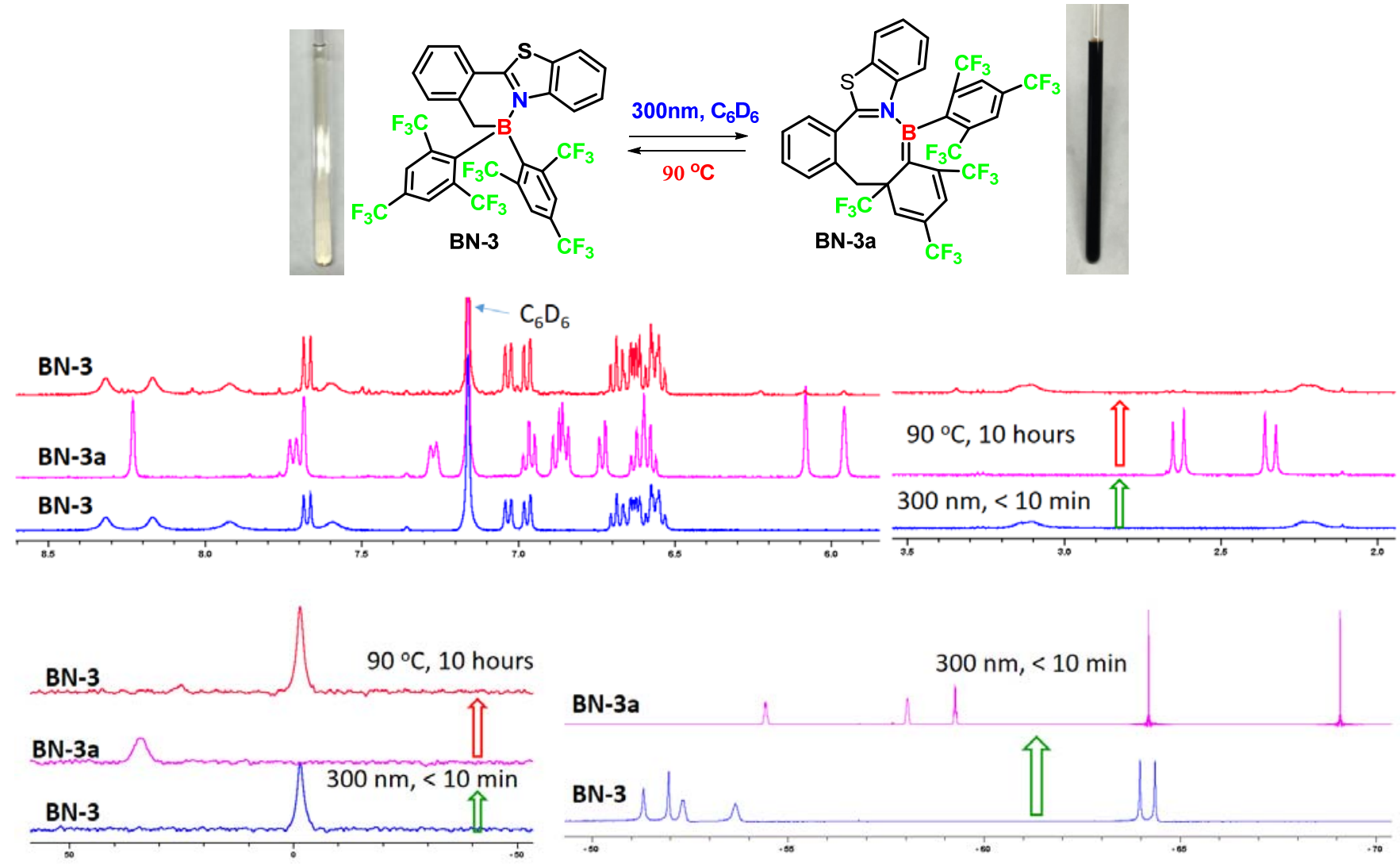

Figure S3.5A. ${ }^{1} \mathrm{H}$ (top), ${ }^{11} \mathrm{~B}$ (bottom left), ${ }^{19} \mathrm{~F}$ (bottom right) NMR spectra showing the clean reversible conversion of $\mathbf{B N - 3}$ (bottom and top spectrum) to $\mathbf{B N - 3 a}$ (middle spectrum) in $\mathrm{C}_{6} \mathrm{D}_{6}$. 
3.6 Photoisomerization of BN-Heterocycle BN-4: Compound BN-4 ( 10 mg) was dissolved in $\mathrm{C}_{6} \mathrm{D}_{6}$ $(0.5 \mathrm{~mL})$ in a quartz J-Young NMR tube and sealed under nitrogen. The NMR tube was then irradiated with $300 \mathrm{~nm}$ UV light and the reaction progress was monitored by ${ }^{1} \mathrm{H}$ and ${ }^{11} \mathrm{~B}$ NMR spectra until it reached completion. The product $\mathbf{B N}-\mathbf{4 b}$ was isolated as pale yellow colored crystals from the solution of benzene/hexanes quantitatively. The structure of $\mathbf{B N}-\mathbf{4} \mathbf{b}$ was confirmed by X-ray diffraction analysis.

BN-4b: ${ }^{1} \mathrm{H}$ NMR $\left(400 \mathrm{MHz}, \mathrm{C}_{6} \mathrm{D}_{6}\right) \delta 8.86(\mathrm{dt}, J=6.3,2.0 \mathrm{~Hz}, 1 \mathrm{H}), 6.83(\mathrm{td}, J=7.6,1.4 \mathrm{~Hz}, 1 \mathrm{H}), 6.74$ (ddd, $J=15.3,7.6,1.5 \mathrm{~Hz}, 2 \mathrm{H}$ ), 6.42 (ddd, $J=7.8,6.5,1.5 \mathrm{~Hz}, 2 \mathrm{H}$ ), 6.27 (dd, $J=7.7,1.5 \mathrm{~Hz}, 1 \mathrm{H}$ ), 6.04 $(\mathrm{dd}, J=7.7,1.3 \mathrm{~Hz}, 1 \mathrm{H}), 3.90(\mathrm{dd}, J=15.0,1.3 \mathrm{~Hz}, 1 \mathrm{H}), 2.24(\mathrm{~d}, J=15.0 \mathrm{~Hz}, 1 \mathrm{H}) .{ }^{13} \mathrm{C}$ NMR $(101$ $\left.\mathrm{MHz}, \mathrm{C}_{6} \mathrm{D} 6\right) \delta 156.56,145.86,145.63,141.12,137.34,135.30,130.68,130.49,126.52,126.16,125.47$, $123.78,123.75,28.41$. (Due to the coupling of fluoride to carbon, carbon atoms connected to fluoride did not show up) ${ }^{19} \mathrm{~F}$ NMR (376 MHz, $\left.\mathrm{C}_{6} \mathrm{D}_{6}, \delta\right):-131.18--131.79(\mathrm{~m}),-132.53--133.47(\mathrm{~m}),-139.87$ (dd, $J=23.9,10.6 \mathrm{~Hz}),-165.63--166.53(\mathrm{~m}) .{ }^{11} \mathrm{~B}$ NMR $\left(128 \mathrm{MHz}, \mathrm{C}_{6} \mathrm{D}_{6}, \delta\right): 5.57$. HR-EIMS (m/z): $[\mathrm{M}]^{+}$calcd. for $\mathrm{C}_{24} \mathrm{H}_{10} \mathrm{BNF} \mathrm{F}_{10}$ : 513.0747; found: 513.0765 .

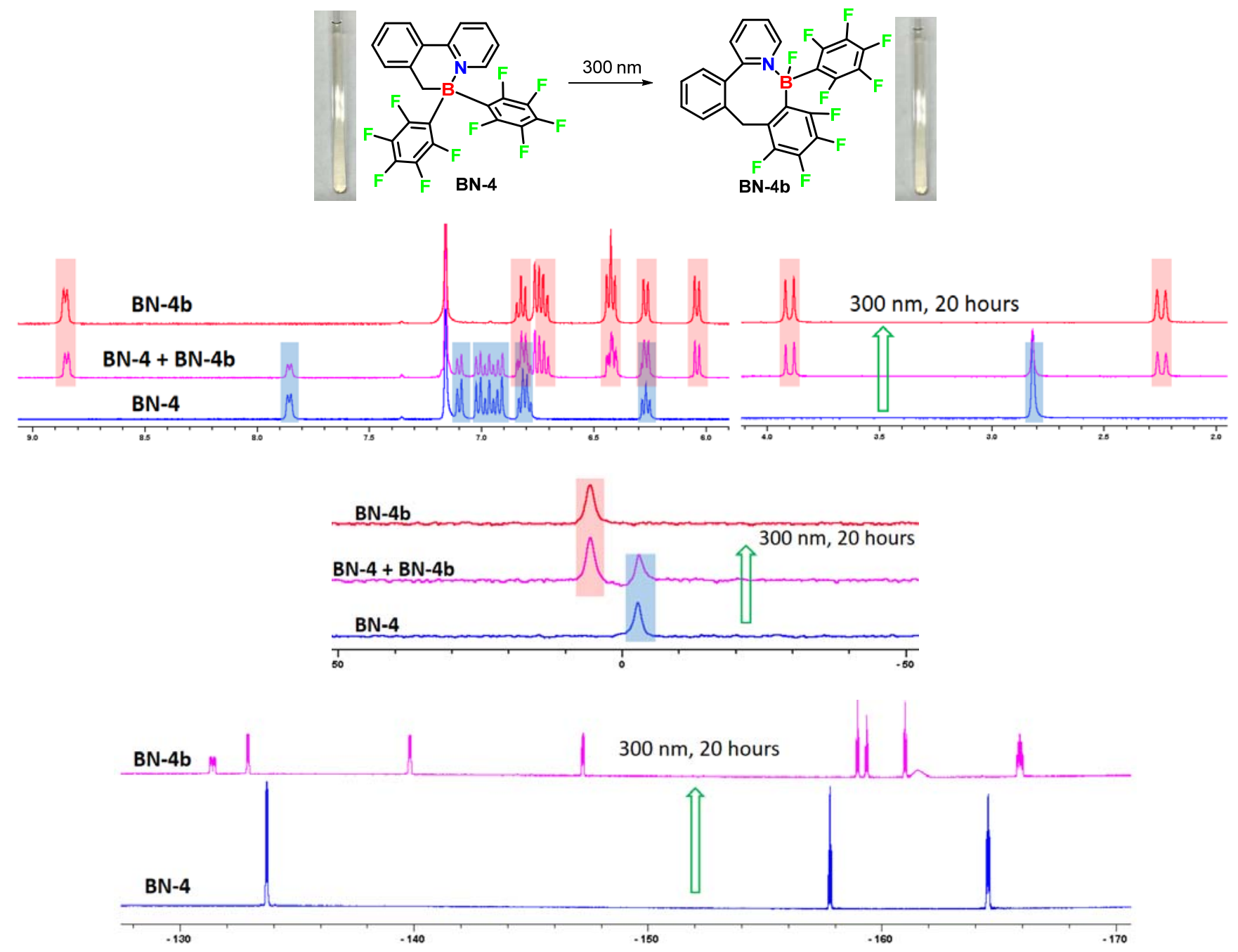

Figure S3.6A. ${ }^{1} \mathrm{H}$ (top), ${ }^{11} \mathrm{~B}$ (middle), ${ }^{19} \mathrm{~F}$ (bottom) NMR spectra showing the clean conversion of BN-4 (bottom spectrum, blue highlight) to $\mathbf{B N}-4 \mathbf{b}$ (top spectrum, red highlight) in C6D6 at room temperature and $300 \mathrm{~nm}$ irradiation. 


\subsection{Photoisomerization quantum yield of $\mathrm{BN}-2$ and $\mathrm{BN}-3$}

The photoisomerization quantum yield of $\mathbf{B N - 2}$ and $\mathbf{B N - 3}$ were determined using $\mathrm{B}(\mathrm{ppy}) \mathrm{Mes}_{2}$ as the reference $\left(\Phi_{\mathrm{PE}}=0.86\right)$.
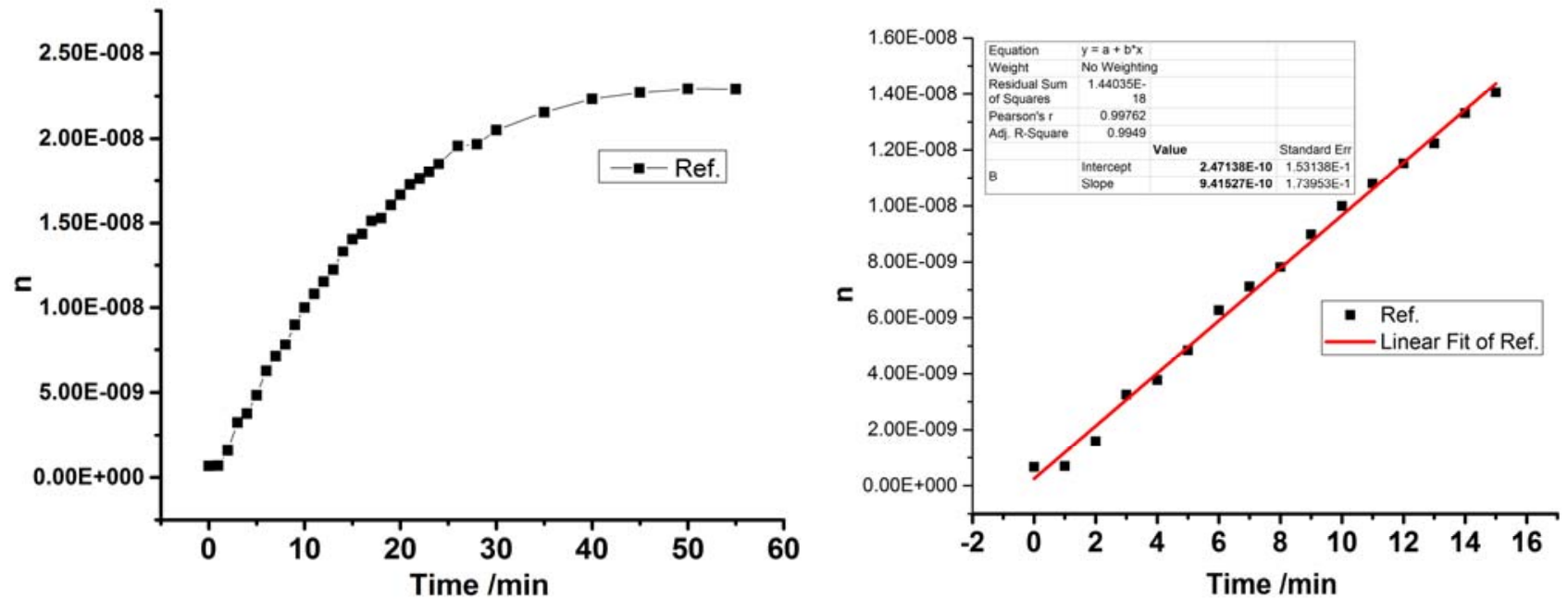

Figure S3.7A. The photoisomerization kinetic data for B(ppy)Mes $\mathrm{Me}_{2}$ in toluene.
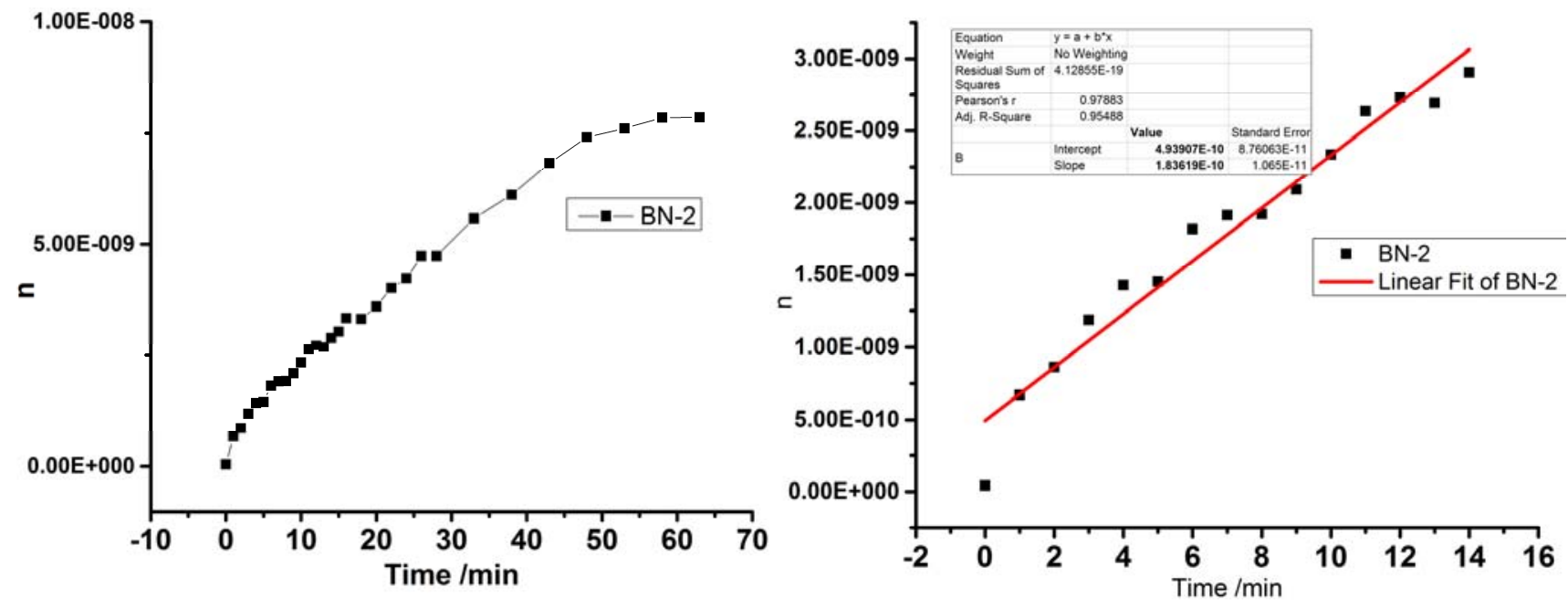

Figure S3.7B. The photoisomerization kinetic data for $\mathbf{B N}-2$ in toluene. 

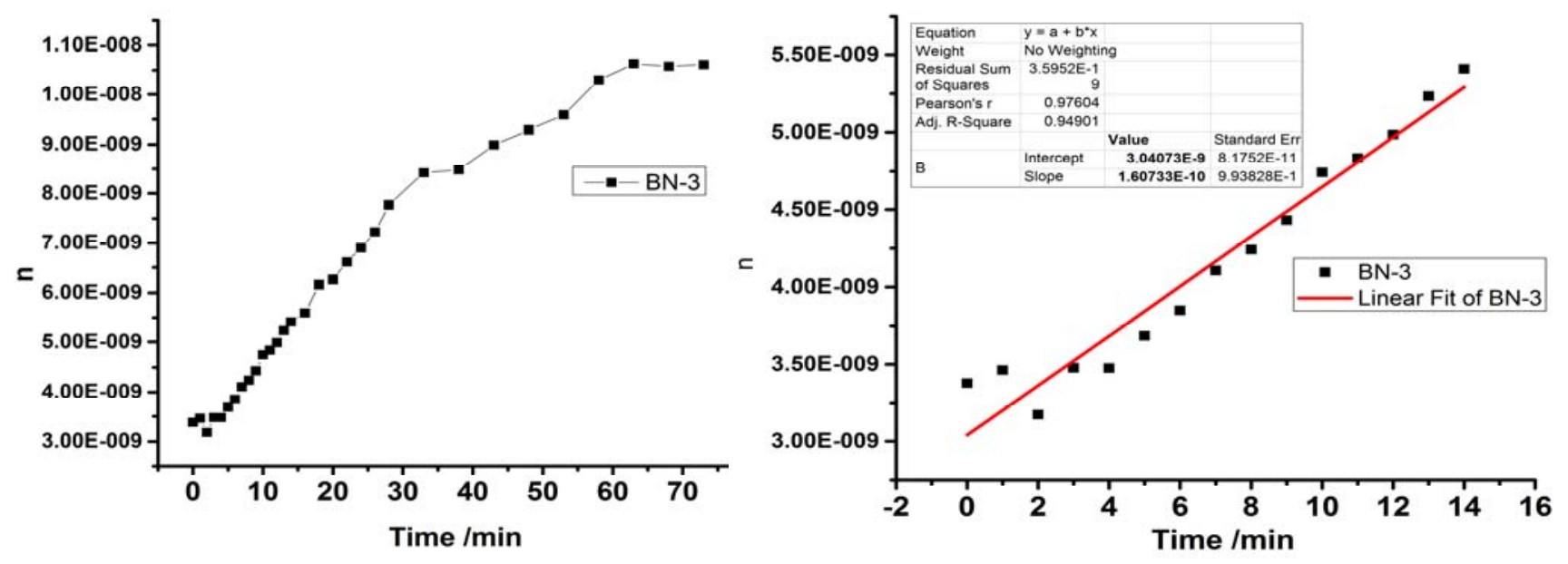

Figure S3.7C. The photoisomerization kinetic data for $\mathbf{B N}-3$ in toluene. 


\section{Photophysical Properties}

\subsection{Photophysical properties of BN-phen-1}

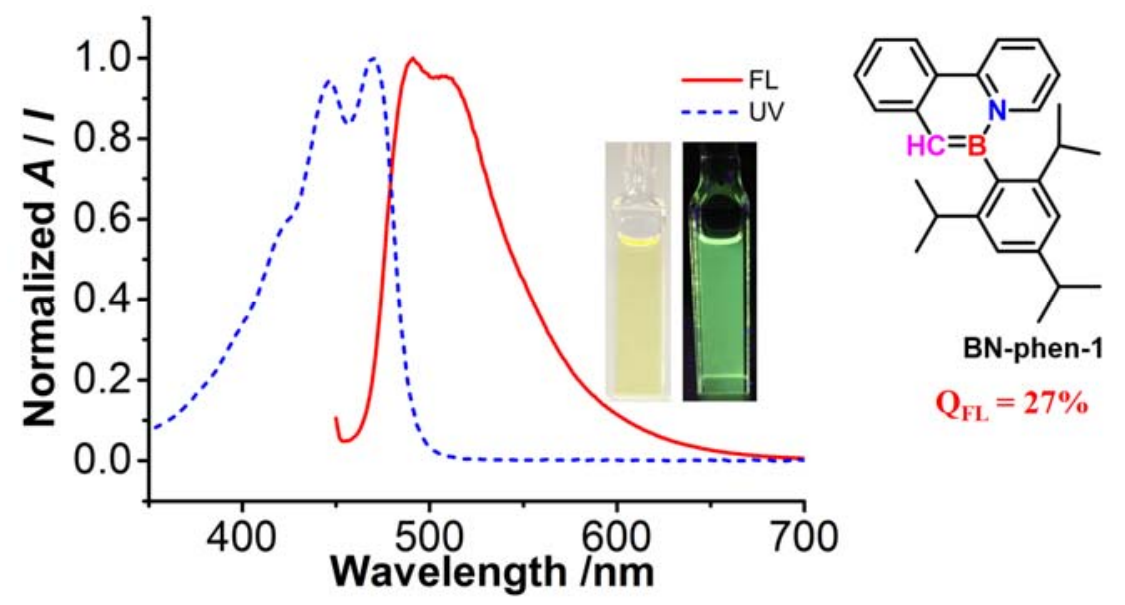

Figure S4.1A. UV/Vis (dot line) and fluorescence (solid line) spectra of BN-phen-1 in THF $\left(1.0 \times 10^{-4} \mathrm{M}\right)$ and the fluorescent quantum efficiency of TIP azaborine BN-phen-1 is 0.27 in THF.

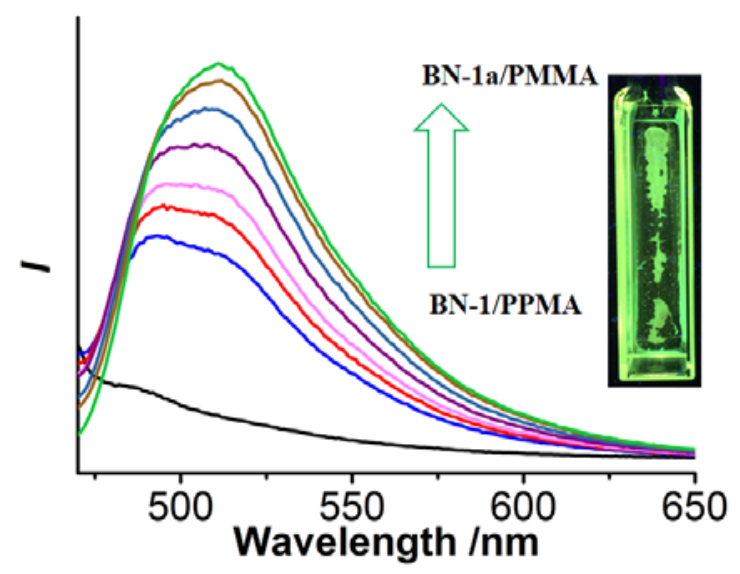

Figure S4.1B. Fluorescence spectra showing the conversion of BN-1 in a PMMA film (20 wt $\%)$ to azaborine BNphen-1. 


\section{2-4.3 Absorption spectra of $\mathrm{BN}-2$ and $\mathrm{BN}-2 \mathrm{a}, \mathrm{BN}-3$ and $\mathrm{BN}-3 \mathrm{a}, \mathrm{BN}-4$ and $\mathrm{BN}-4 \mathrm{a}$}
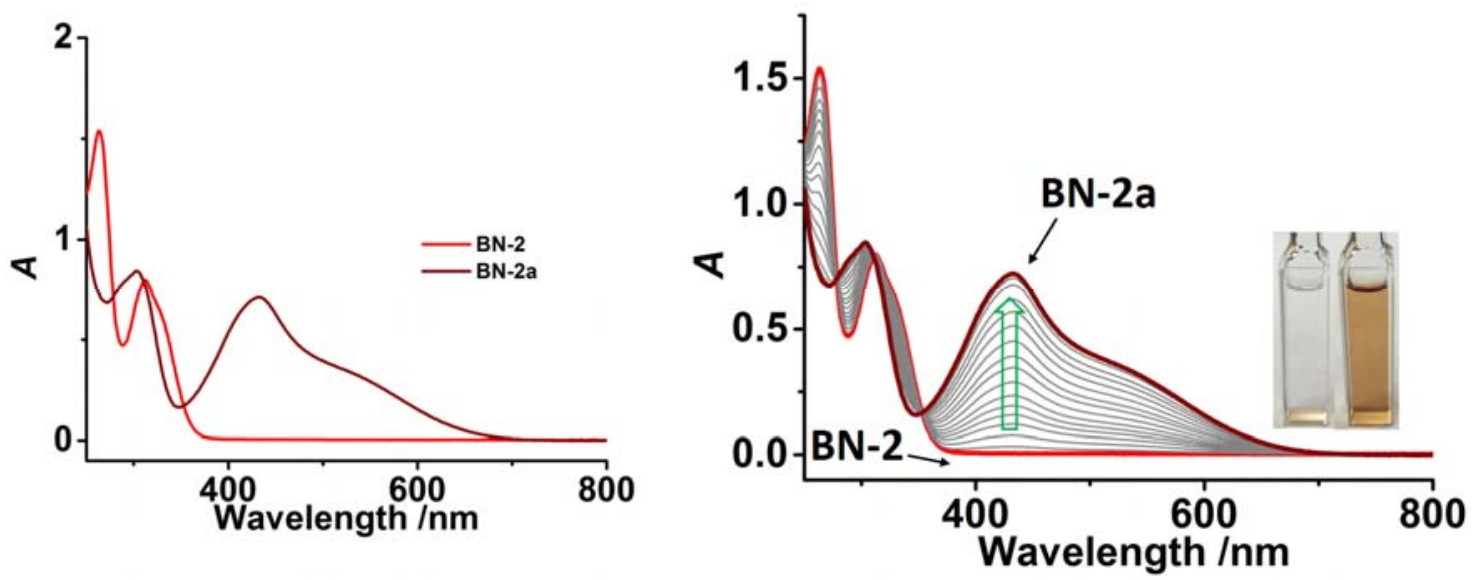

Figure S4.2A. Left: UV/Vis spectra of BN-2 and BN-2a in THF $\left(1.0 \times 10^{-4} \mathrm{M}\right)$. Right: UV/Vis spectra showing the conversion of $\mathbf{B N}-\mathbf{2}$ in THF to $\mathbf{B N}-\mathbf{2} \mathbf{a}$.
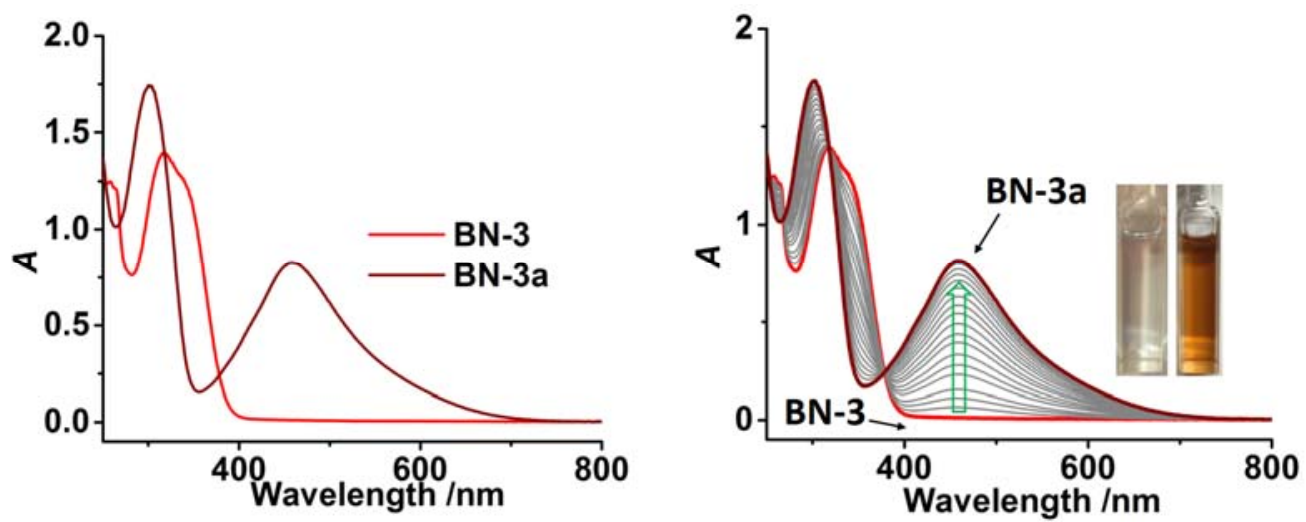

Figure S4.3A. Left: UV/Vis spectra of $\mathbf{B N - 3}$ and $\mathbf{B N - 3 a}$ in THF $\left(1.0 \times 10^{-4} \mathrm{M}\right)$. Right: UV/Vis spectra showing the conversion of $\mathbf{B N}-\mathbf{3}$ in THF to $\mathbf{B N}-\mathbf{3 a}$.
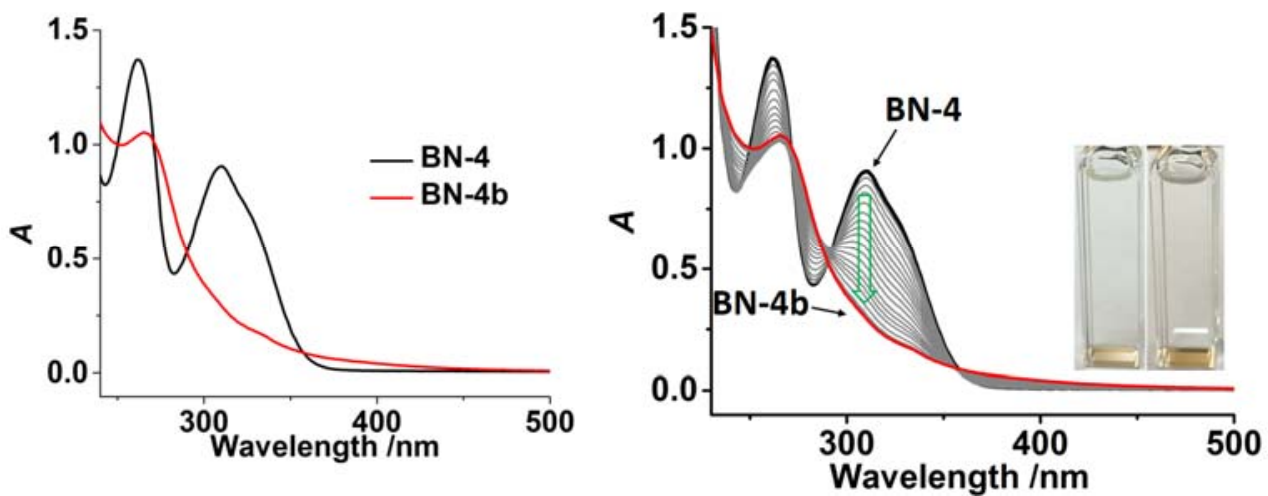

Figure S4.3B. Left: UV/Vis spectra of $\mathbf{B N}-\mathbf{4}$ and $\mathbf{B N}-\mathbf{4 b}$ in THF $\left(1.0 \times 10^{-4} \mathrm{M}\right)$. Right: UV/Vis spectra showing the conversion of $\mathbf{B N}-4$ in THF to $\mathbf{B N}-\mathbf{4 b}$. 
V. NMR Data

a. ${ }^{1} \mathrm{H}(400 \mathrm{MHz})$ and ${ }^{13} \mathrm{C}(101 \mathrm{MHz}) \mathrm{NMR}$ of $\mathrm{BN}-H e t e r o c y c l e ~ B N-1$ in $\mathrm{C}_{6} \mathrm{D}_{6}$ at $298 \mathrm{~K}$
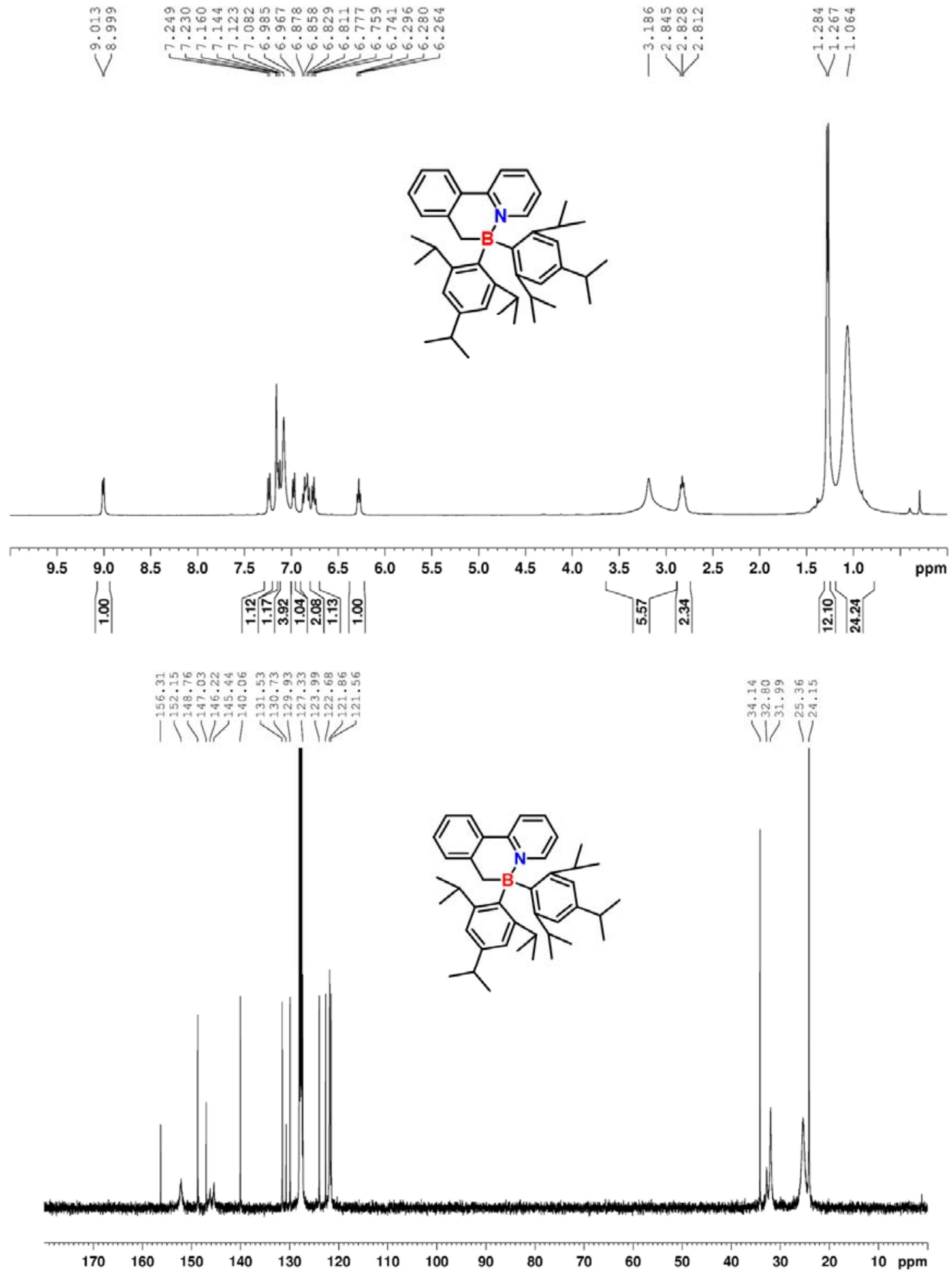
b. ${ }^{1} \mathrm{H}(400 \mathrm{MHz}),{ }^{13} \mathrm{C}(101 \mathrm{MHz})$ and ${ }^{19} \mathrm{~F}\left(376 \mathrm{MHz}, \mathrm{C}_{6} \mathrm{D}_{6}\right)$ NMR of BN-Heterocycle $\mathrm{BN}-2$ in $\mathrm{CDCl}_{3}$ at $298 \mathrm{~K}$

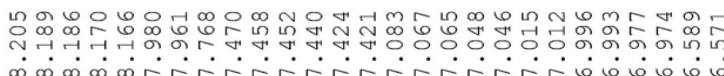
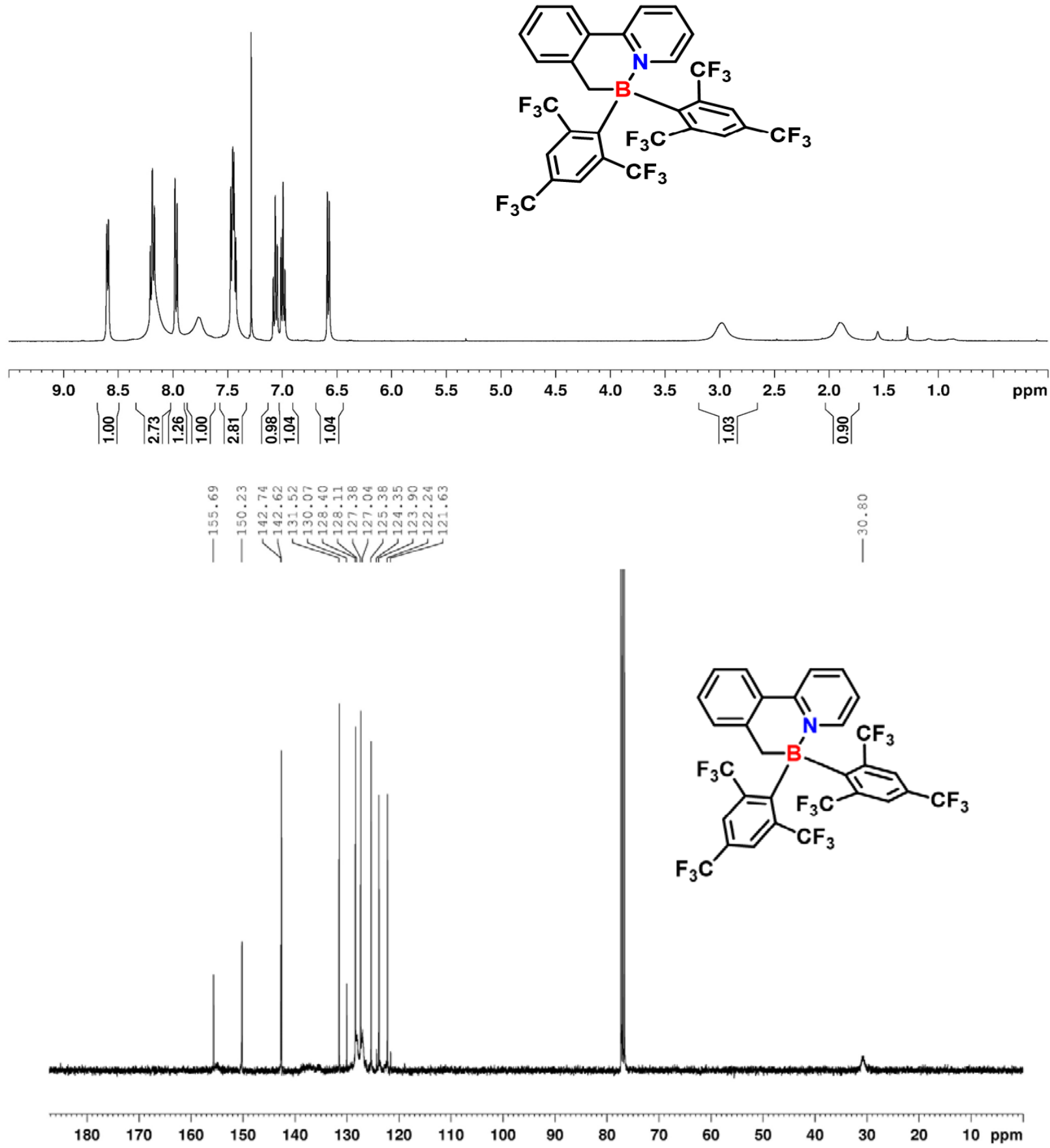
c. ${ }^{1} \mathrm{H}\left(499 \mathrm{MHz}, \mathrm{C}_{6} \mathrm{D}_{6}\right)$ and ${ }^{13} \mathrm{C}\left(126 \mathrm{MHz}, \mathrm{CDCl}_{3}\right) \mathrm{NMR}$ of $\mathrm{BN}-H e t e r o c y c l e ~ B N-2$ at 278K

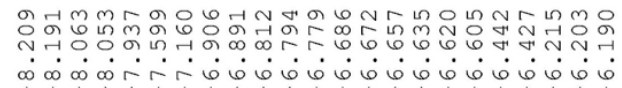

$\rightarrow 1$

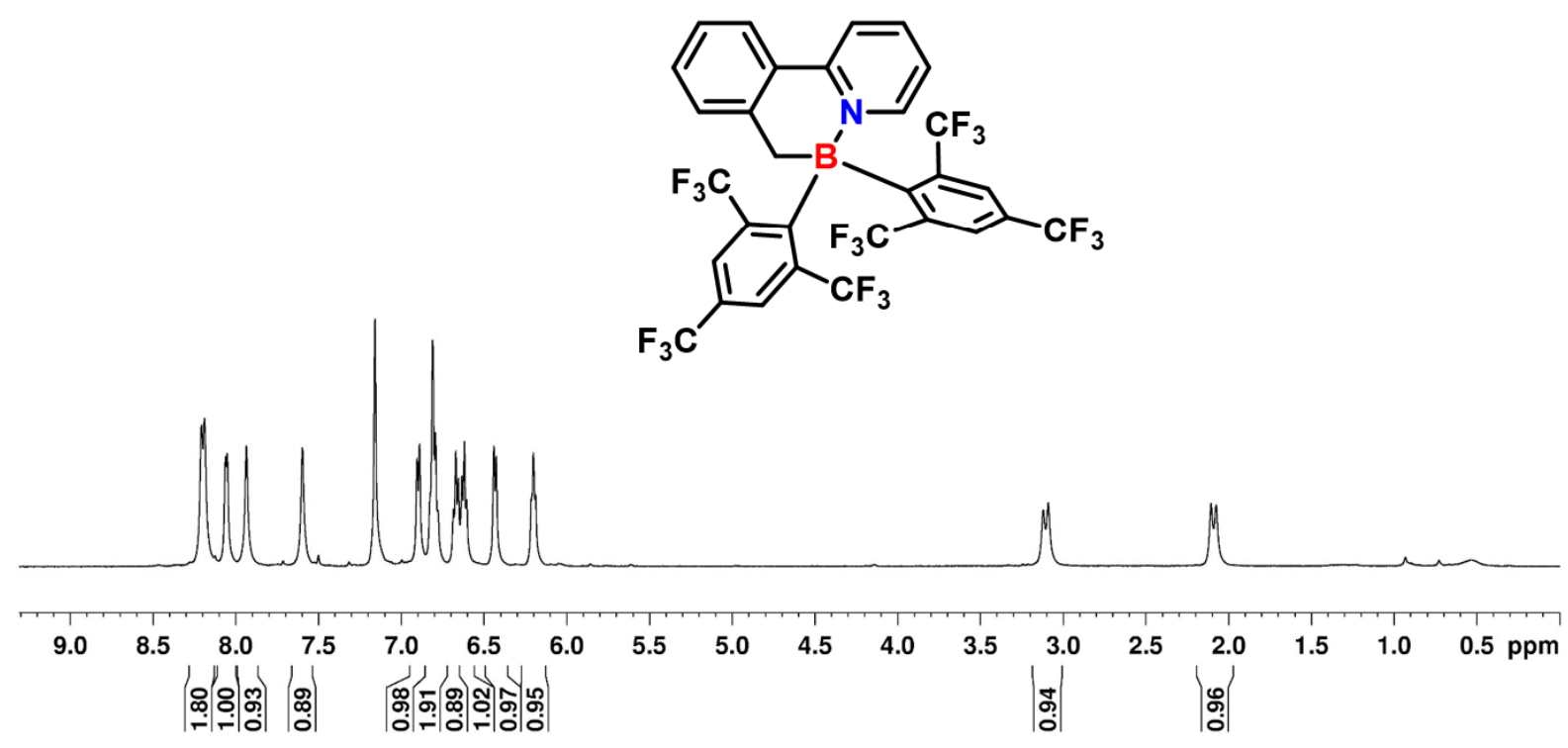

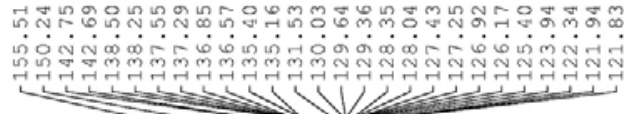

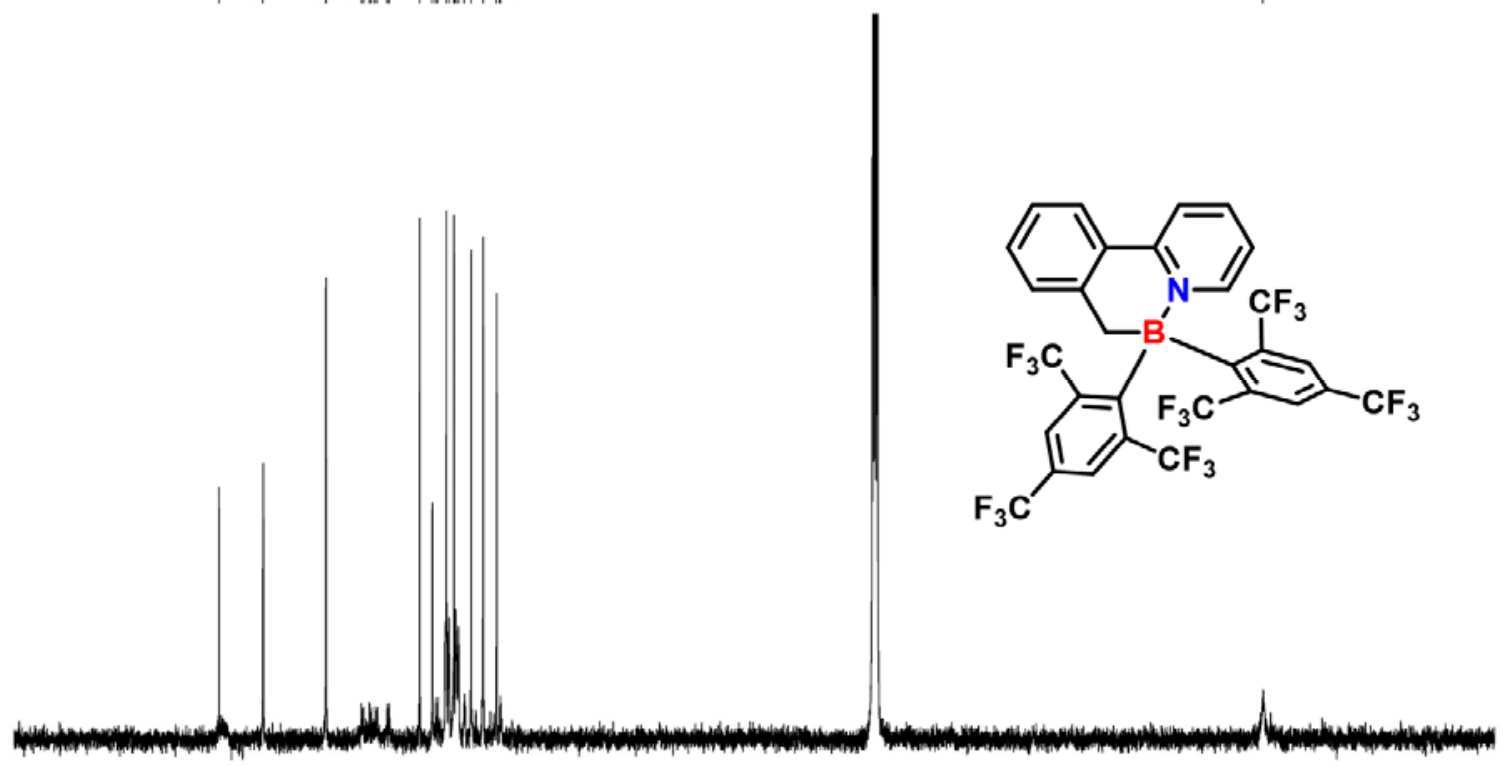

$\begin{array}{lllllllllllllllll}170 & 160 & 150 & 140 & 130 & 120 & 110 & 100 & 90 & 80 & 70 & 60 & 50 & 40 & 30 & 20 & \mathrm{ppm}\end{array}$ 
d. ${ }^{1} \mathrm{H}(400 \mathrm{MHz})$ and ${ }^{13} \mathrm{C}(101 \mathrm{MHz}) \mathrm{NMR}$ of $\mathrm{BN}-H e t e r o c y c l e ~ B N-3$ in $\mathrm{CD}_{2} \mathrm{Cl}_{2}$ at $298 \mathrm{~K}$

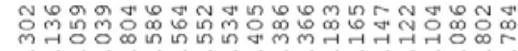

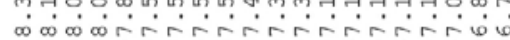

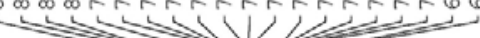
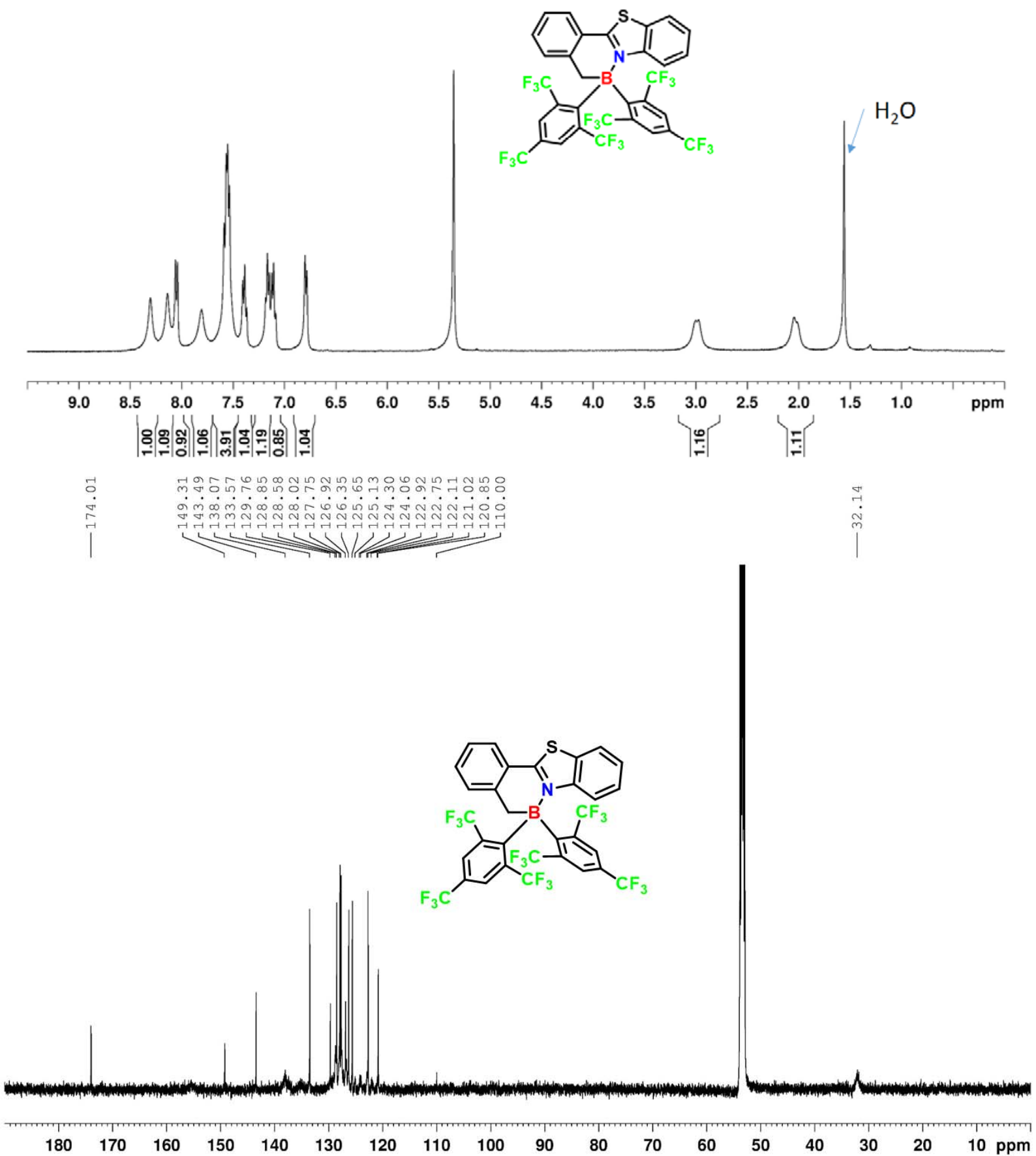


\section{e. ${ }^{1} \mathrm{H}\left(400 \mathrm{MHz}, \mathrm{C}_{6} \mathrm{D}_{6}\right)$ and ${ }^{13} \mathrm{C}\left(101 \mathrm{MHz}, \mathrm{CD}_{2} \mathrm{Cl}_{2}\right)$ NMR of BN-Heterocycle BN-5 at 298K}
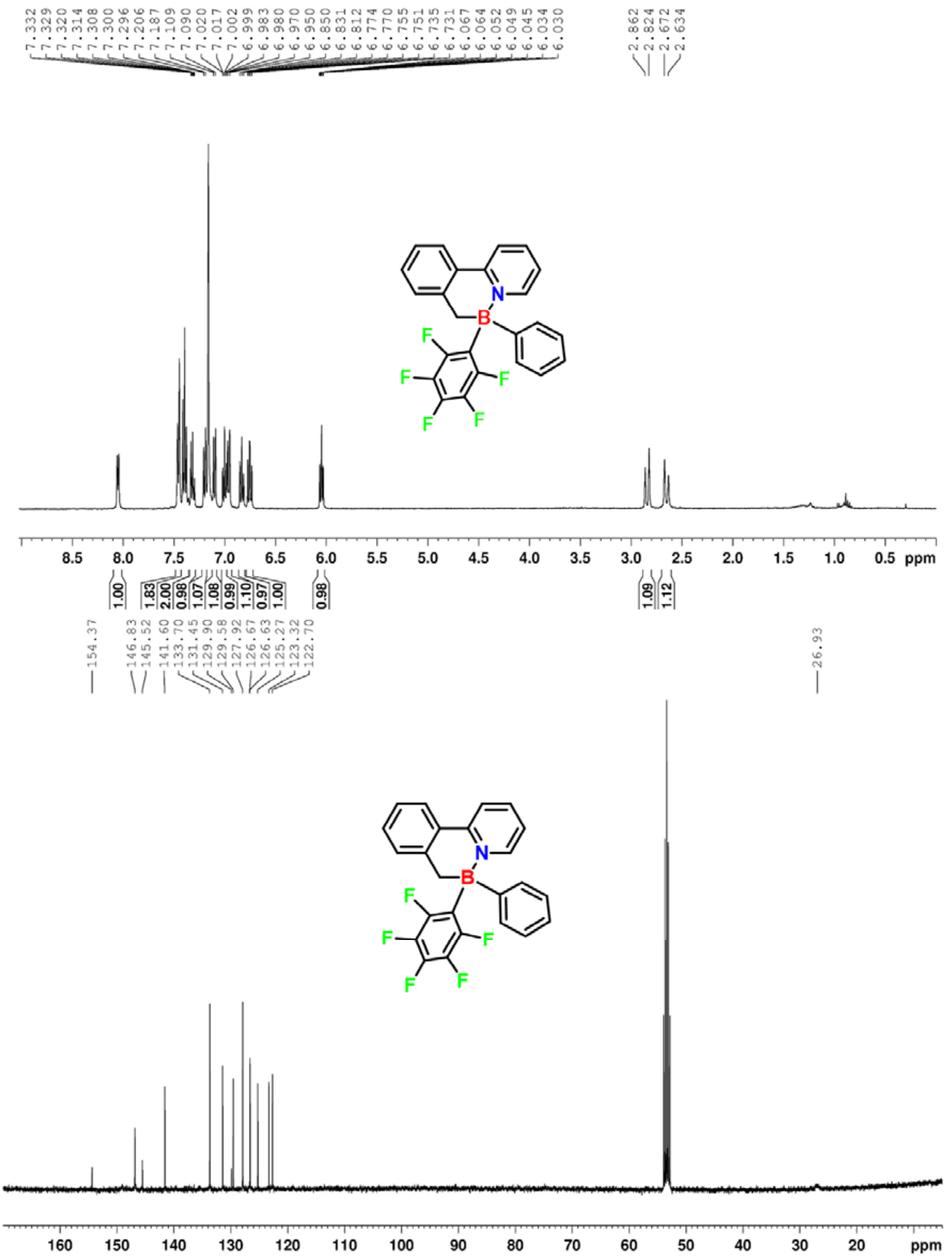
f. ${ }^{1} \mathrm{H}(400 \mathrm{MHz})$ and ${ }^{13} \mathrm{C}(101 \mathrm{MHz}) \mathrm{NMR}$ of $\mathrm{BN}-H e t e r o c y c l e ~ B N-6$ in $\mathrm{CDCl}_{3}$ at $298 \mathrm{~K}$

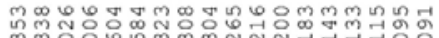

$\infty \infty \dot{\infty} \infty$

$\rightarrow+\infty$
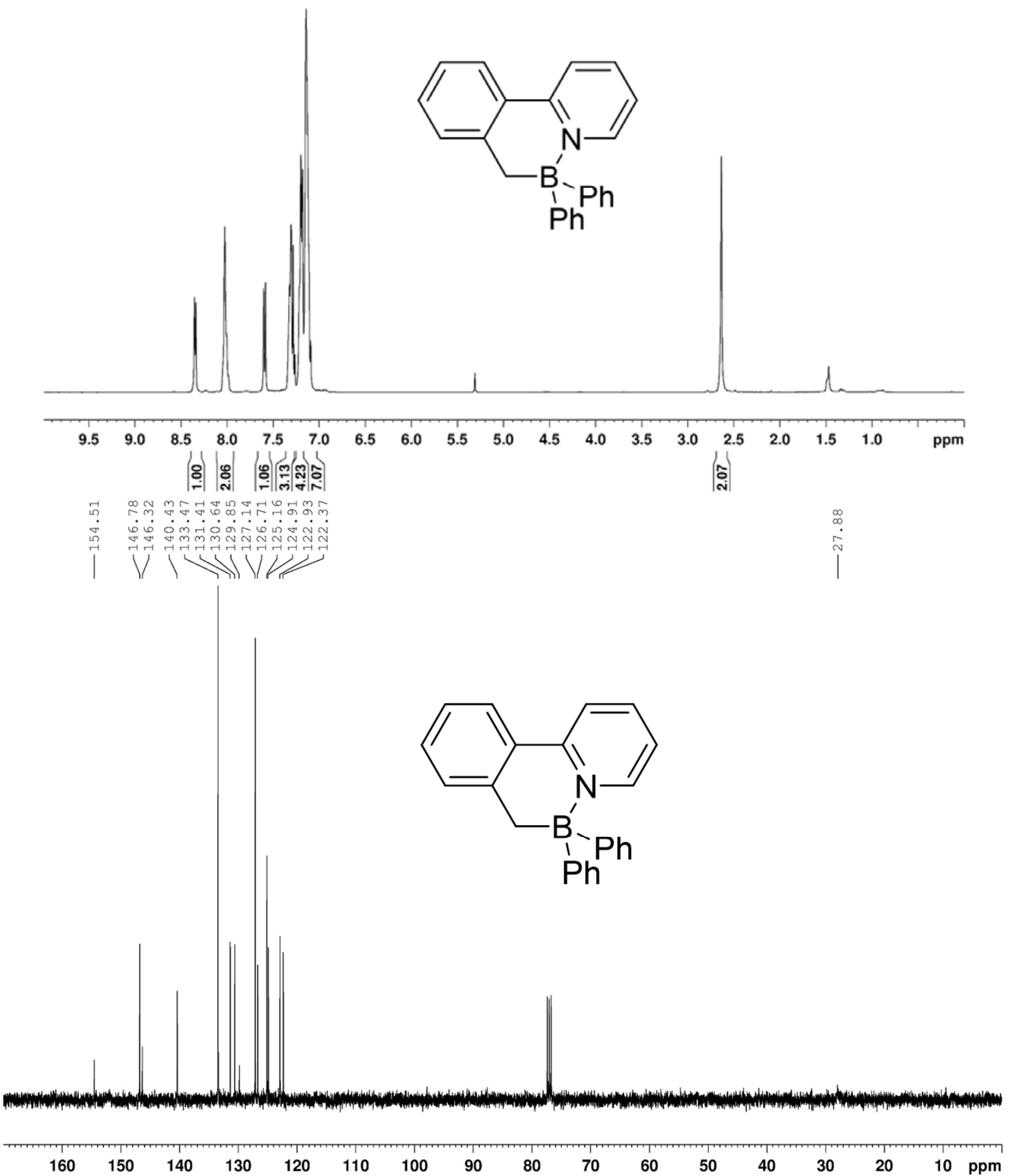
g. ${ }^{1} \mathrm{H}(500 \mathrm{MHz})$ and ${ }^{13} \mathrm{C}(101 \mathrm{MHz})$ NMR of Compound BN-phen-1 in $\mathrm{C}_{6} \mathrm{D}_{6}$ at $298 \mathrm{~K}$

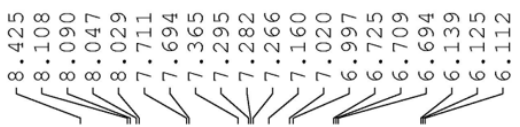

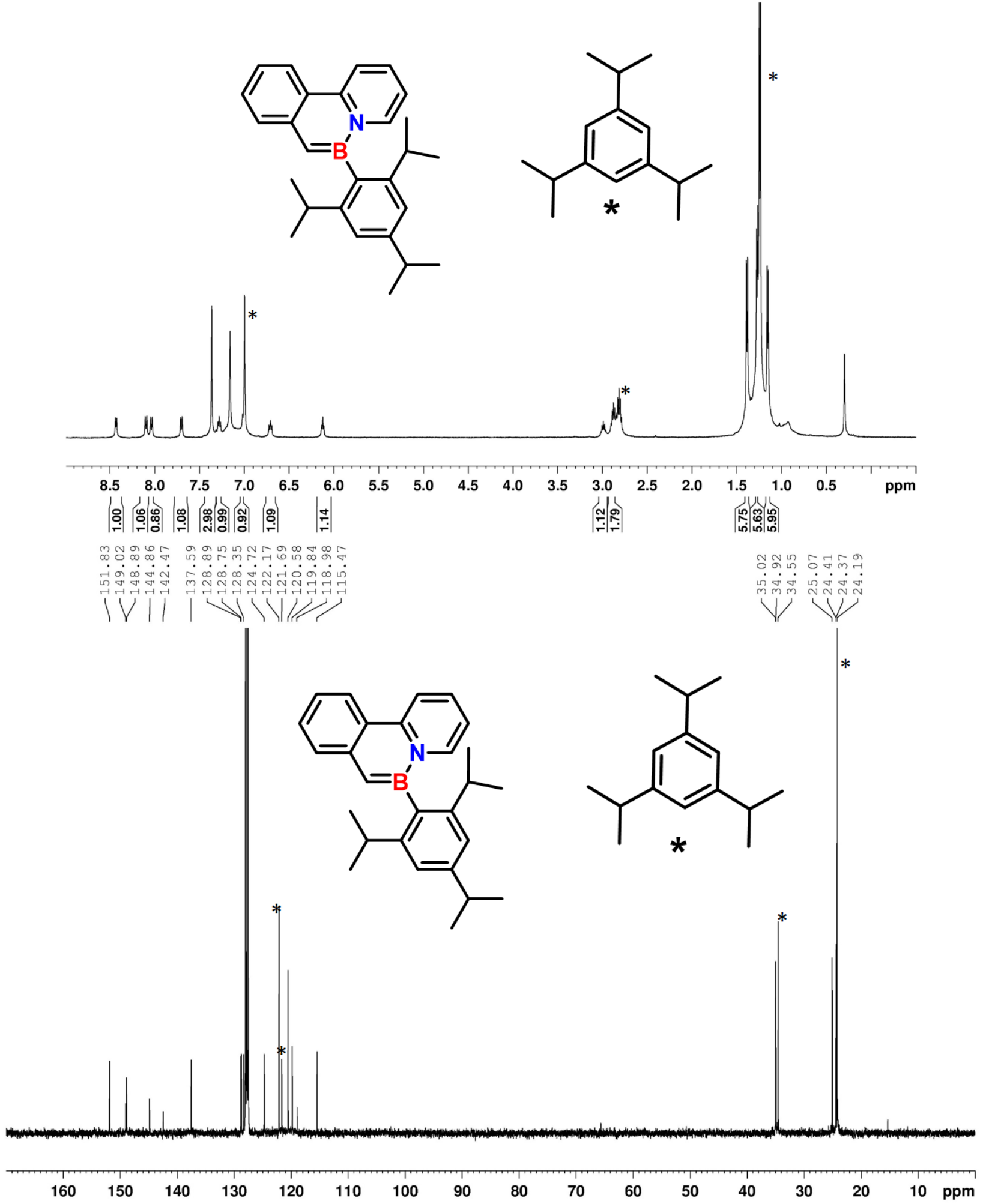


h. ${ }^{1} \mathrm{H}(400 \mathrm{MHz})$ and ${ }^{13} \mathrm{C}(101 \mathrm{MHz})$ NMR of Compound $\mathrm{BN}-2 \mathrm{a}$ in $\mathrm{C}_{6} \mathrm{D}_{6}$ at $298 \mathrm{~K}$

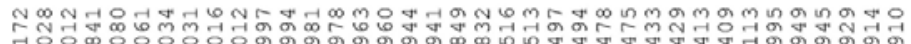

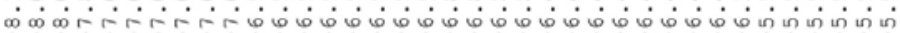
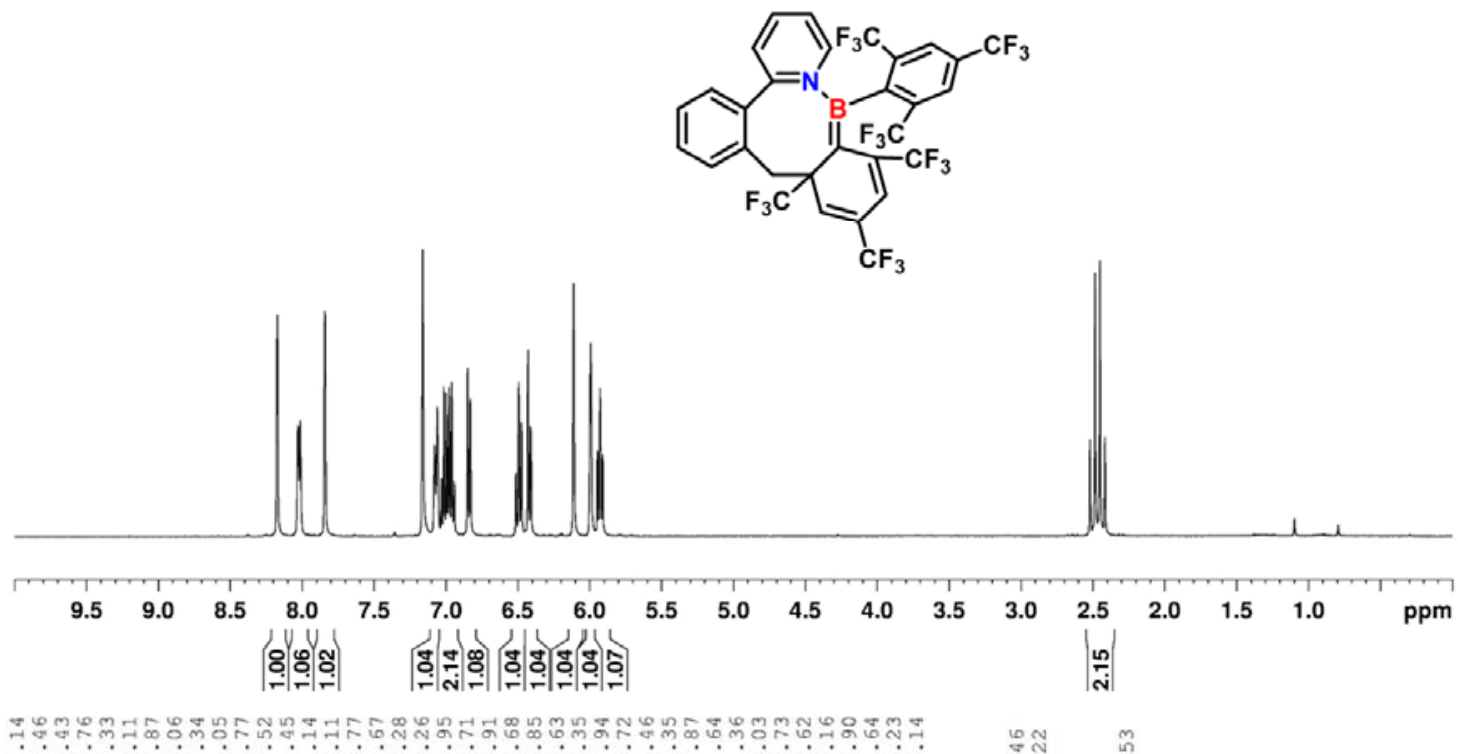

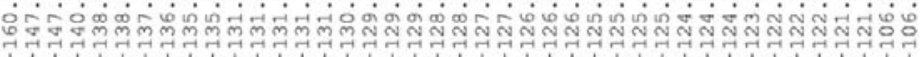
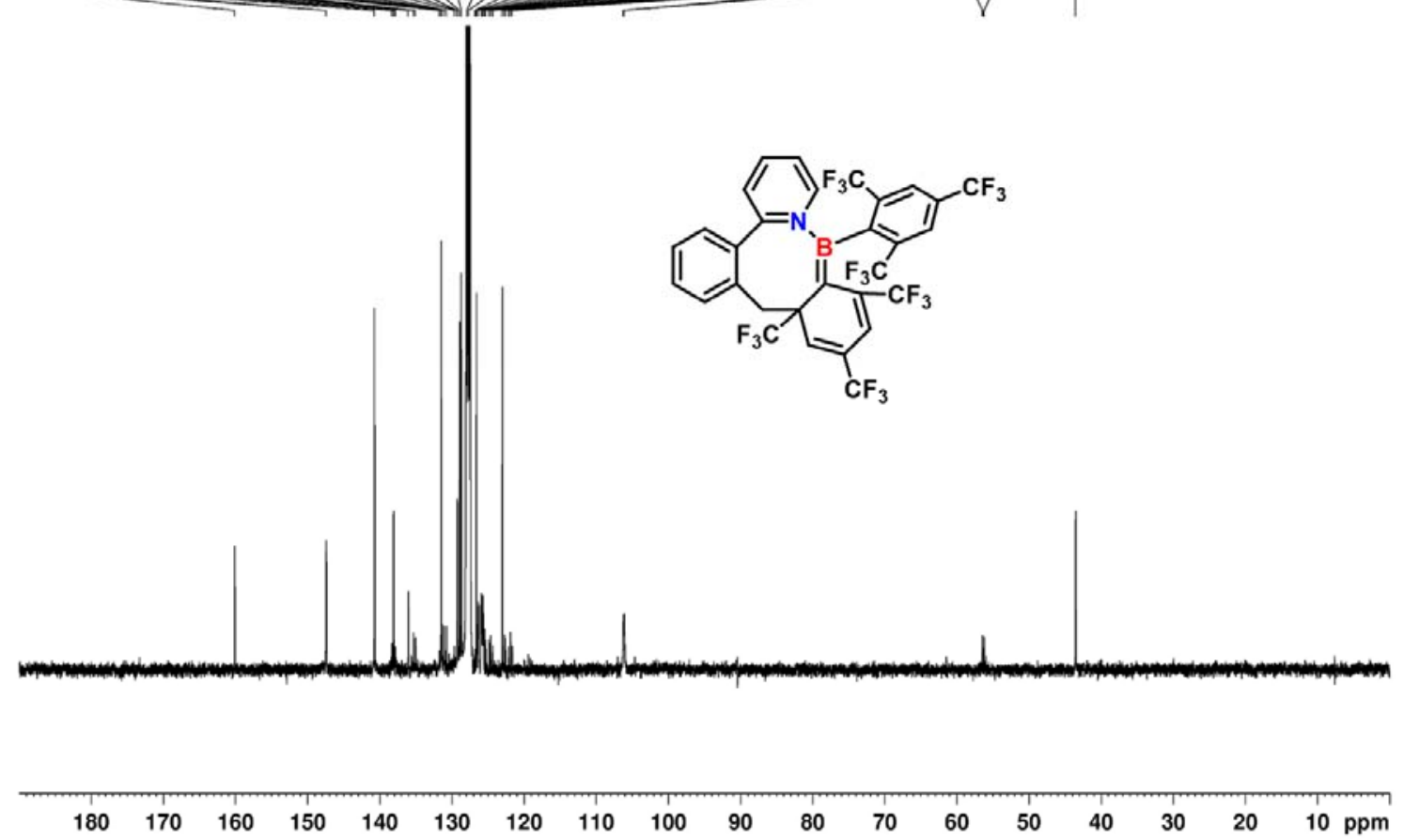
i. $\quad{ }^{1} \mathrm{H}(500 \mathrm{MHz})$ and ${ }^{13} \mathrm{C}(126 \mathrm{MHz}) \mathrm{NMR}$ of Compound $\mathrm{BN}-3 \mathrm{a}$ in $\mathrm{C}_{6} \mathrm{D}_{6}$ at $298 \mathrm{~K}$

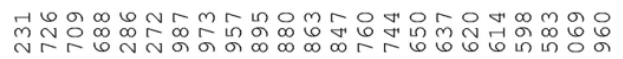

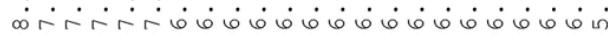

$\mathrm{N}^{2}$

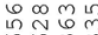

$60 \mathrm{~m}$

जंत्रि
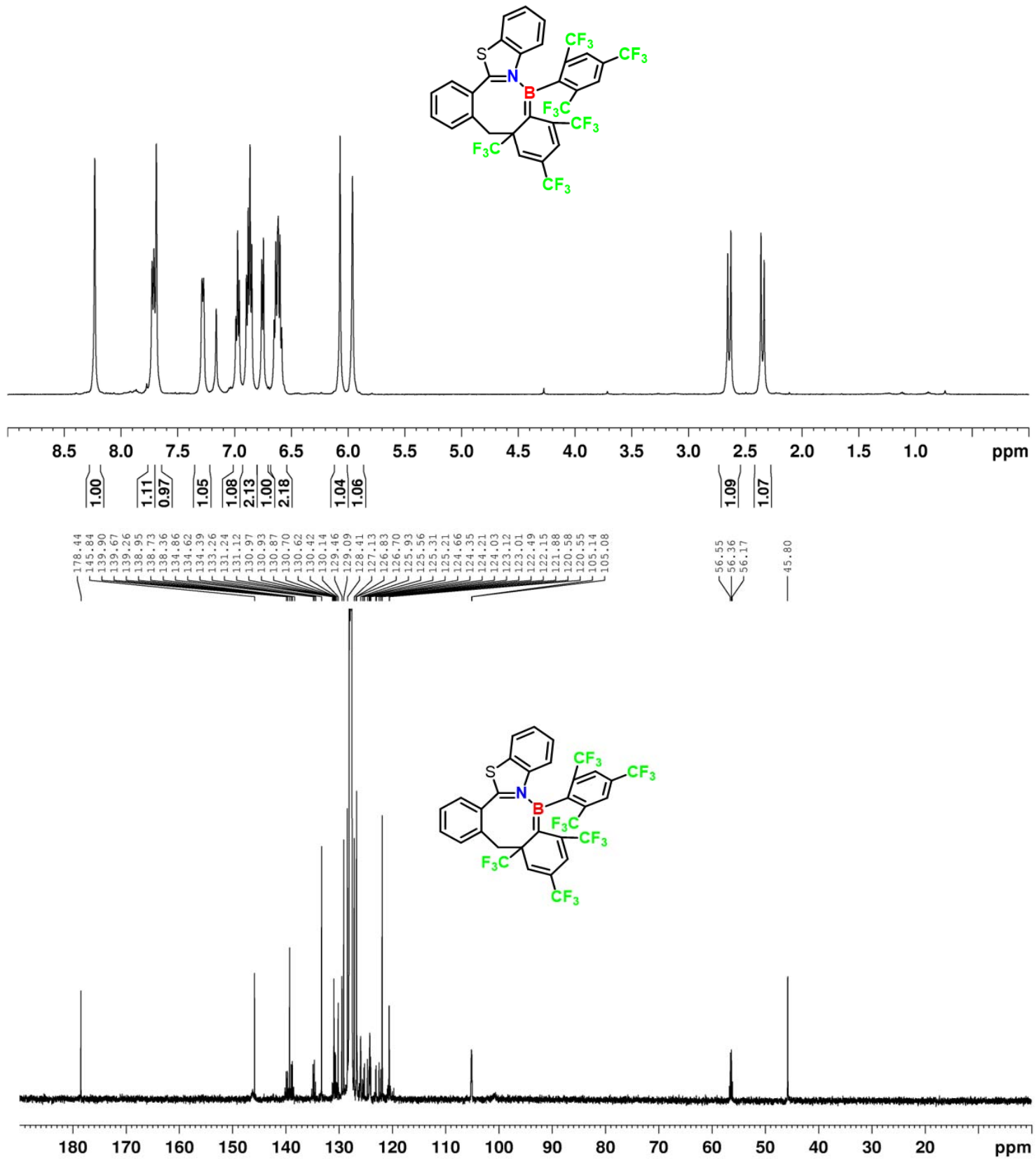
j. $\quad{ }^{1} \mathrm{H}(400 \mathrm{MHz})$ and ${ }^{13} \mathrm{C}(101 \mathrm{MHz})$ NMR of Compound BN-4b in $\mathrm{C}_{6} \mathrm{D}_{6}$ at $298 \mathrm{~K}$

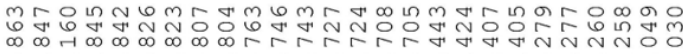
$\dot{\infty} \dot{r} \dot{\omega} \dot{\omega} \dot{\omega} \dot{\omega} \dot{\omega} \dot{\omega} \dot{\omega} \dot{\omega} \dot{\omega} \dot{\omega} \dot{\omega} \dot{\omega} \dot{\omega} \dot{\omega} \dot{\omega} \dot{\omega} \dot{\omega} \dot{\omega}$ $\mathrm{N}^{\infty}$

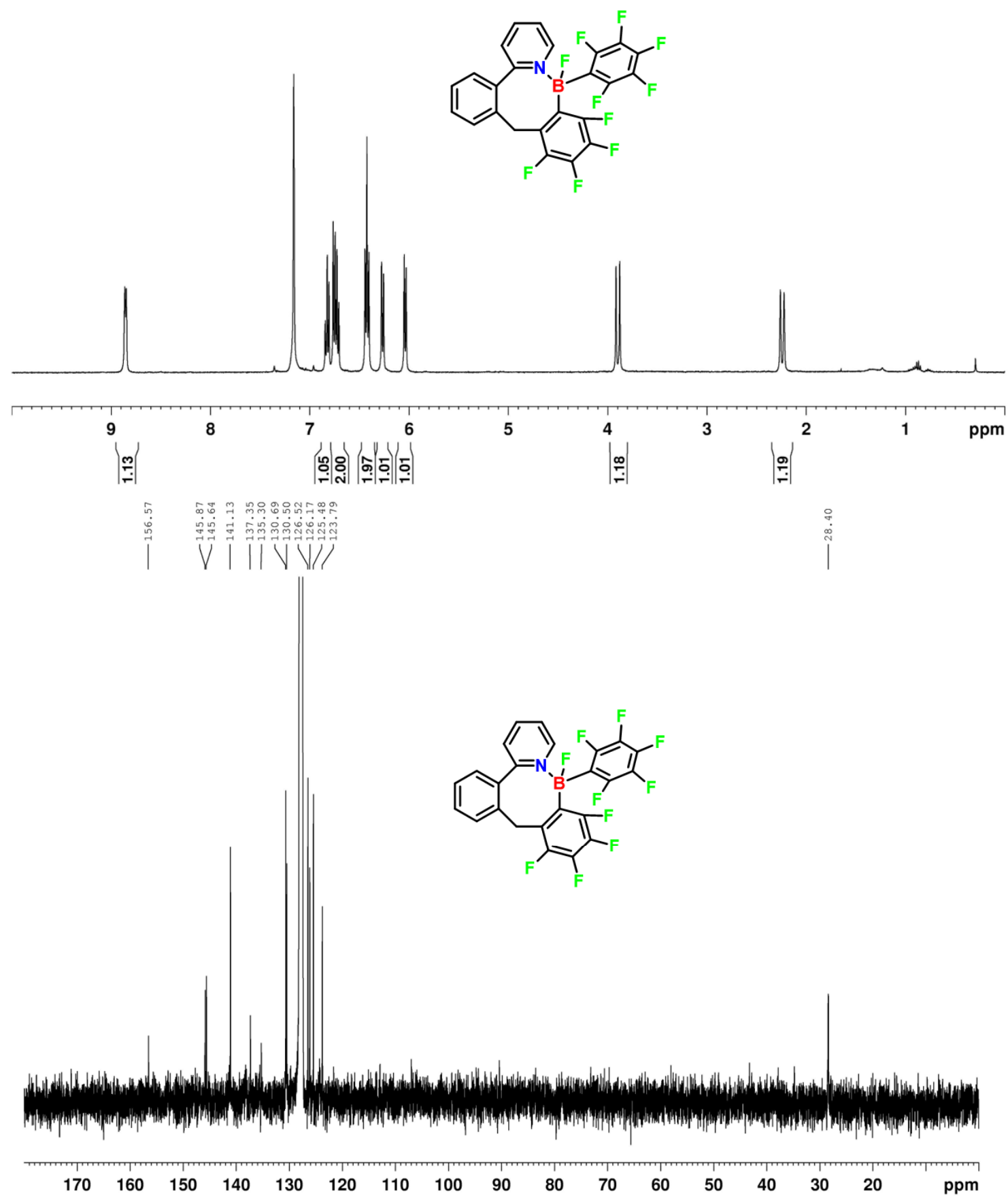




\section{Computational Results}

All calculations were performed using density functional theory (DFT) with standard 6-31g* basis set implemented in Gaussian 09 package. ${ }^{5}$ The X-ray structures of reactant and product were used as starting geometries for ground-state $\left(\mathrm{S}_{0}\right)$ optimization. Geometry optimizations in ground state were carried out with hybrid functional B3LYP. ${ }^{6-8}$ In the optimizations, dispersion corrections were considered using the empirical formula by Grimme et al. (i.e., DFT-D3). ${ }^{9-12}$ Analytical frequency calculations were performed to confirm the nature of stationary points (minima or transition states) at the same theory level as geometry optimizations. Critical points in the first excited singlet state $\left(\mathrm{S}_{1}\right)$ were optimized with long-range corrected hybrid exchange-correlation functional using the Coulombattenuating method CAM-B3LYP. ${ }^{13}$ Vertical excitation energies at the $\mathrm{S}_{0}$ minima were computed by means of time-dependent DFT method with CAM-B3LYP functional. The reaction path calculations were started from the transition state structure following the vector with a negative eigenvalue in forward and reverse directions, using intrinsic reaction coordinate (IRC) approach. ${ }^{14,15}$ The IRC provides the minimum energy path connecting the two local minima of the reactant and product sides via a given transition state structure. This allows for easy understanding of complicated multistep mechanisms as a set of simple elementary reaction steps. To account for the solvent effects, the benzene solvent was employed by using the conducting polarizable continuum model $(\mathrm{C}-\mathrm{PCM})^{16-18}$ in all calculations.

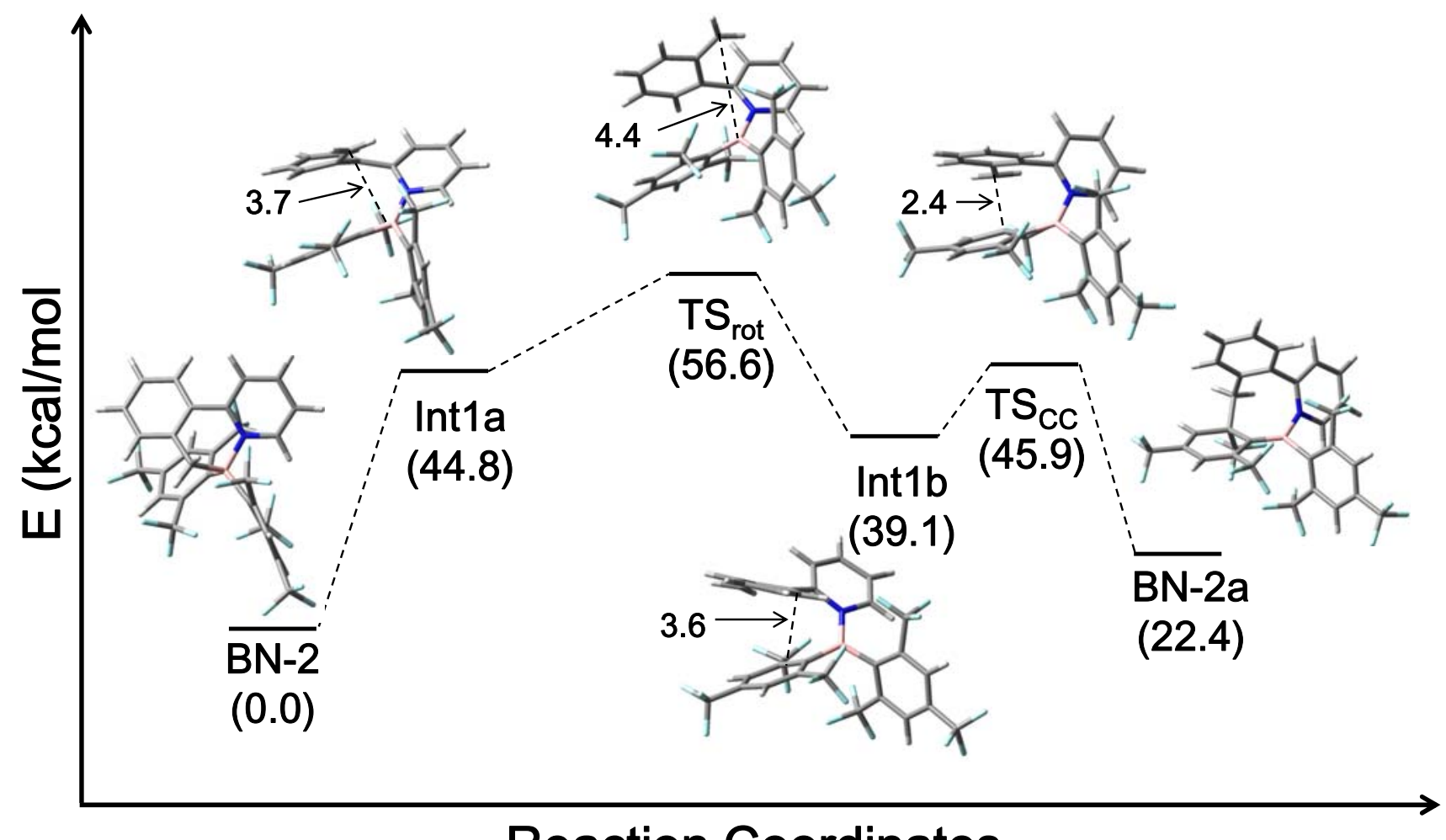

\section{Reaction Coordinates}

Figure S6.1A. Calculated potential energy profile for the photoisomerization of BN-2. The energies of the all key species are reported relative to $\mathbf{B N - 2}$ and given in the parentheses. Some key distances (in $\AA$ ) are also shown. 


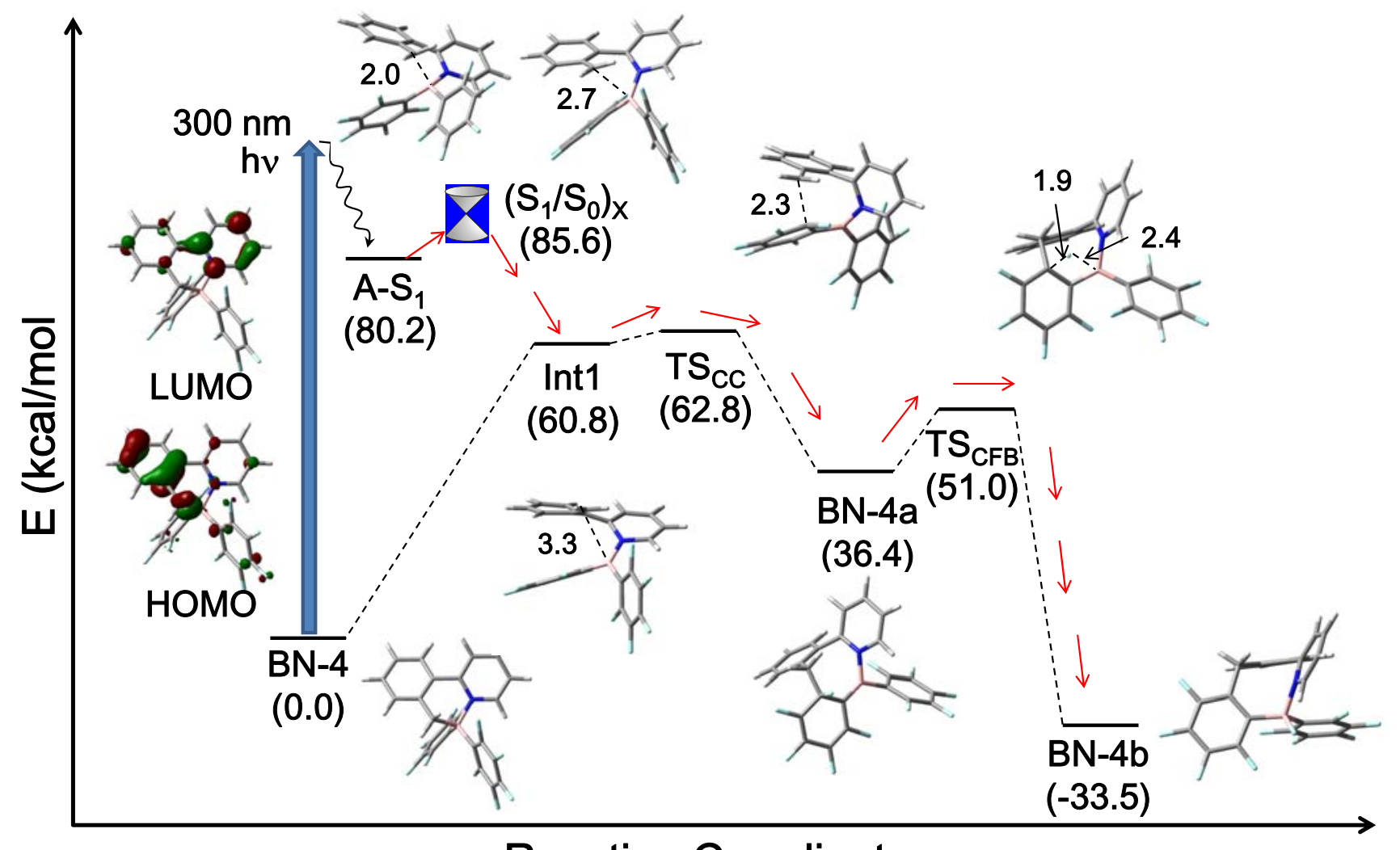

Reaction Coordinates

Figure S6.1B. Calculated potential energy profile for the photoisomerization of BN-4. The energies of the all key species, as well as the intersection point of the $\mathrm{S}_{0}$ and $\mathrm{S}_{1}$ state $\left(\left(\mathrm{S}_{1} / \mathrm{S}_{0}\right)_{\mathrm{X}}\right)$ are relative to $\mathbf{B N}-\mathbf{4}$ and given in the parentheses. Some key distances (in $\AA$ ) and the HOMO/LUMO orbitals of BN-4 are also shown.
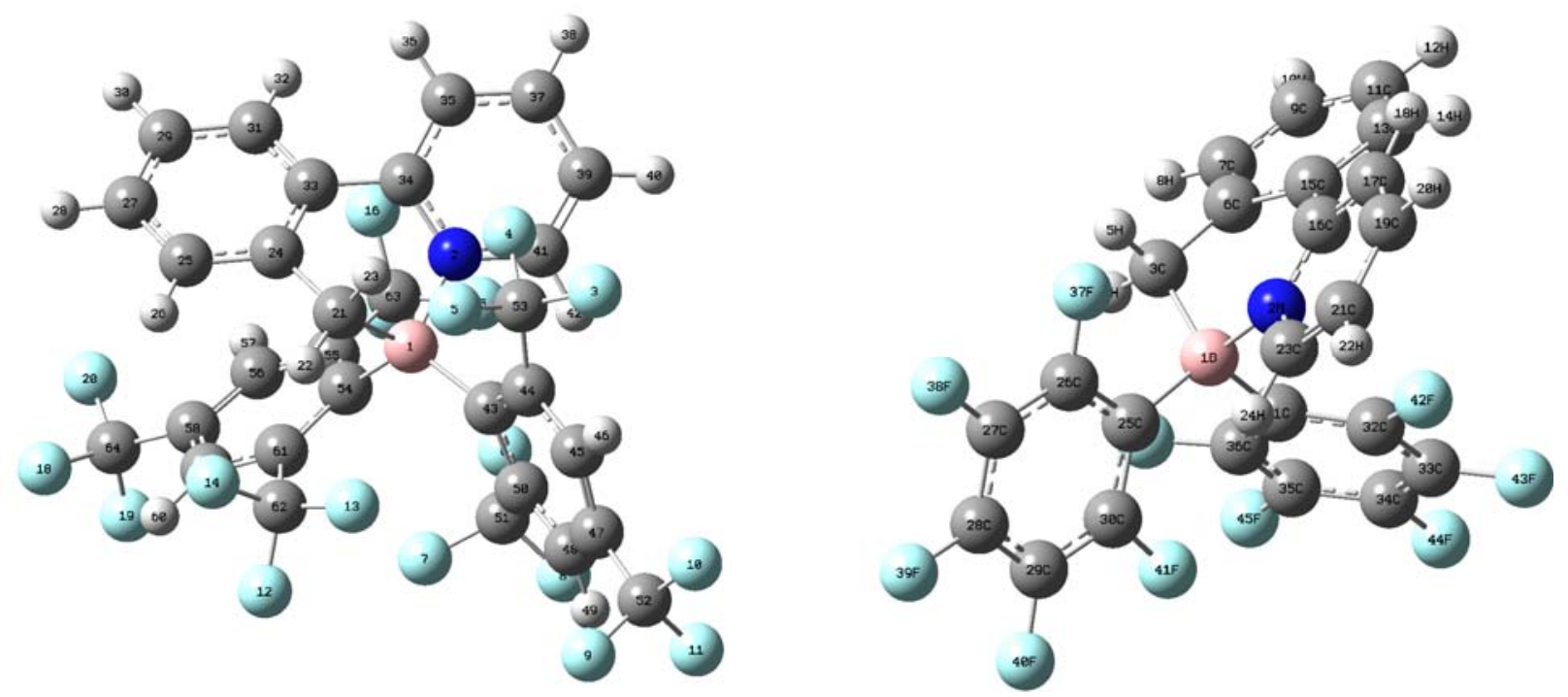

Figure S6.1C. Atomic symbols and labels of BN-2 (left) and BN-4 (right) at the Franck-Condon geometry. 


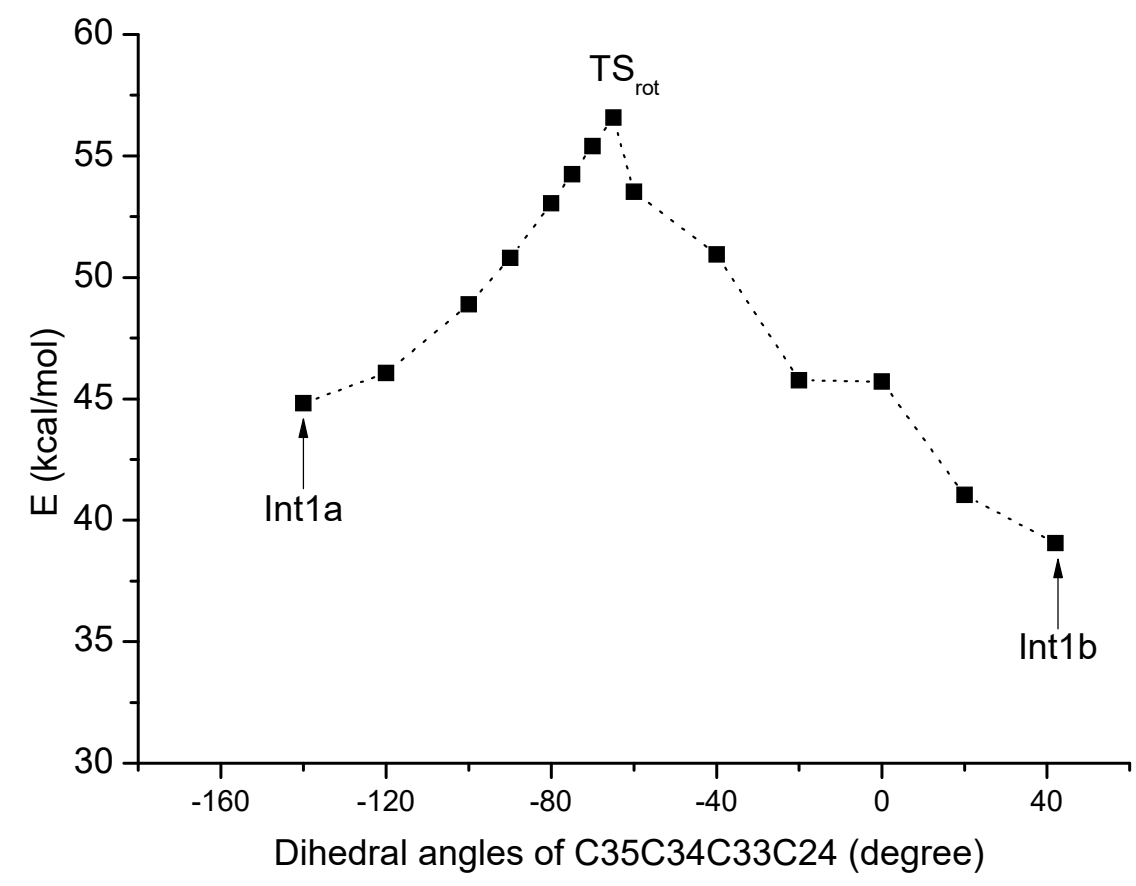

Figure S6.1D. Energy profile from Intla to Int1b shown in Figure S6.1 a by fixing the dihedral of C35C34C33C24 (see Figure S6.1C. for atomic labeling). Calculations performed at B3LYP-D3/6-31g* level of theory with benzene as the solvent. Energies are reported relative to reactant $\mathbf{B N}-\mathbf{2}$ in the ground state. 


\begin{tabular}{|c|c|c|}
\hline & HOMO & LUMO \\
\hline \multicolumn{3}{|l|}{$\mathrm{BN}-0$} \\
\hline \multicolumn{3}{|l|}{ BN-1 } \\
\hline \multicolumn{3}{|l|}{ BN-2 } \\
\hline \multicolumn{3}{|l|}{ BN-4 } \\
\hline \multicolumn{3}{|l|}{ BN-5 } \\
\hline & & \\
\hline
\end{tabular}

Figure S6.1D2. Comparison of HOMO and LUMO diagrams for BN-0, BN-1, BN-2, BN-4 and BN-5 obtained at B3LYP/6-31g* level in benzene. 
Table 6.1. Cartesian coordinates of the optimized structures in reaction pathway calculations

\begin{tabular}{|c|c|c|c|}
\hline \multicolumn{4}{|c|}{$\begin{array}{l}\text { Part 1: Optimized structures for BN-2 } \\
\text { BN-2 }\end{array}$} \\
\hline B & -0.20052600 & 0.65412600 & -0.16028200 \\
\hline $\mathbf{N}$ & 0.02852400 & 2.10777400 & 0.56607300 \\
\hline$F$ & -4.11081500 & 2.73624700 & -1.15784500 \\
\hline $\mathrm{F}$ & -1.99816100 & 3.01705700 & -0.88936000 \\
\hline $\mathrm{F}$ & -2.74123800 & 1.93051700 & -2.62853300 \\
\hline $\mathrm{F}$ & -0.41676100 & -0.81240500 & 2.52710400 \\
\hline $\mathrm{F}$ & -0.46759300 & -2.60075700 & 1.27084300 \\
\hline $\mathrm{F}$ & -1.92266400 & -2.32095200 & 2.84264300 \\
\hline $\mathrm{F}$ & -5.77610300 & -2.59266300 & -0.98852400 \\
\hline $\mathrm{F}$ & -6.76260400 & -0.77600300 & $0 \quad-0.31297600$ \\
\hline $\mathrm{F}$ & -6.12033800 & -2.27883900 & 1.13436300 \\
\hline $\mathrm{F}$ & 0.23164100 & -3.49814700 & -1.45405700 \\
\hline $\mathrm{F}$ & -1.00987000 & -1.73732800 & -1.52924900 \\
\hline $\mathrm{F}$ & 0.6 & -1.95313100 & -2.9 \\
\hline $\mathrm{F}$ & 1.06 & 1.11717700 & 300 \\
\hline$F$ & 200 & 2.01 & 600 \\
\hline $\mathrm{F}$ & 3.03075000 & 0.35731500 & 3.2 \\
\hline $\mathrm{F}$ & 6600 & -3.05895500 & -1.3 \\
\hline $\mathrm{F}$ & 00 & -3.64286300 & 0.75303800 \\
\hline $\mathrm{F}$ & & -1.78578500 & 28600 \\
\hline $\mathrm{C}$ & & 1.02708200 & -1.7 \\
\hline $\mathrm{H}$ & -0.0 & 0.19178300 & -2.4 \\
\hline $\mathrm{H}$ & -0.59 & 1.81357000 & -2.09385100 \\
\hline $\mathrm{C}$ & 00 & 1.55051700 & -1.88100100 \\
\hline $\mathrm{C}$ & 00 & 1.07656500 & -2.81798700 \\
\hline $\mathrm{H}$ & 2.08958000 & 0.27929600 & -3.49112000 \\
\hline $\mathrm{C}$ & & 1.59196400 & -2.87 \\
\hline $\mathrm{H}$ & 4.3 & 1.20291400 & 800 \\
\hline $\mathrm{C}$ & 4.08 & 2.59276800 & 6800 \\
\hline $\mathrm{H}$ & 5.096 & 2.98217400 & -2.00500300 \\
\hline $\mathrm{C}$ & 3.18004500 & $3.06905500-1$ & 1.03298100 \\
\hline $\mathrm{H}$ & 3.50627200 & $3.80088800-$ & -0.30027600 \\
\hline $\mathrm{C}$ & 1.87584000 & $2.55350900-0$ & 0.9796 \\
\hline $\mathrm{C}$ & 0.933 & 2.999 & 0.06 \\
\hline $\mathrm{C}$ & 0.966 & 4.305 & 0.567 \\
\hline $\mathrm{H}$ & 1.65253300 & 5.01570000 & 0.12294400 \\
\hline $\mathrm{C}$ & 0.12599000 & 4.68663900 & 1.60237800 \\
\hline $\mathrm{H}$ & 0.15460400 & 5.70220300 & 1.98585100 \\
\hline $\mathrm{C}$ & -0.75537500 & 3.74625000 & 2.13588700 \\
\hline $\mathrm{H}$ & -1.42659000 & 3.98548200 & 2.95256900 \\
\hline $\mathrm{C}$ & -0.77488400 & 2.48045700 & 1.58389300 \\
\hline $\mathrm{H}$ & -1.44175500 & 1.71337800 & 1.95121300 \\
\hline $\mathrm{C}$ & -1.79490800 & 0.18026300 & 0.12624300 \\
\hline $\mathrm{C}$ & -2.90780400 & 0.78938600 & -0.53430600 \\
\hline $\mathrm{C}$ & -4.17972200 & 0.20302600 & -0.57098400 \\
\hline $\mathrm{H}$ & -4.97970700 & 0.68534100 & -1.11741900 \\
\hline 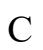 & -4.44196200 & -0.97803200 & 0.09935500 \\
\hline
\end{tabular}

$\begin{array}{cccc}\mathrm{C} & -3.44024400 & -1.52469300 & 0.88396700 \\ \mathrm{H} & -3.65428600 & -2.39947800 & 1.48380600 \\ \mathrm{C} & -2.16878200 & -0.94797800 & 0.91871100 \\ \mathrm{C} & -1.23306300 & -1.66446600 & 1.87636900 \\ \mathrm{C} & -5.78000800 & -1.65467900 & -0.01524100 \\ \mathrm{C} & -2.91412800 & 2.11097700 & -1.29748700 \\ \mathrm{C} & 1.06783200 & -0.40646700 & 0.18501000 \\ \mathrm{C} & 2.16658300 & -0.16911600 & 1.06752700 \\ \mathrm{C} & 3.38723700 & -0.84727300 & 0.94886500 \\ \mathrm{H} & 4.21161000 & -0.59024200 & 1.60109000 \\ \mathrm{C} & 3.56615700 & -1.84740800 & 0.01051600 \\ \mathrm{C} & 2.48219900 & -2.21794000 & -0.76549300 \\ \mathrm{H} & 2.58017300 & -3.04173500 & -1.46275400 \\ \mathrm{C} & 1.27174200 & -1.52846300 & -0.67394700 \\ \mathrm{C} & 0.26255800 & -2.15531000 & -1.62725500 \\ \mathrm{C} & 2.23395000 & 0.82801700 & 2.22197000 \\ \mathrm{C} & 4.87204000 & -2.58284600 & -0.08675800\end{array}$

Int1a

$\mathrm{B}$

$\mathrm{N}$

$\mathrm{F}$

$\mathrm{F}$

$\mathrm{F}$

$\mathrm{F}$

$\mathrm{F}$

$\mathrm{F}$

F

F

F

F

F

F

$\begin{array}{ccc}-0.20642700 & 0.89332600 & 0.31385400 \\ 0.09902500 & 2.28726200 & 0.49057000 \\ -3.49812800 & 2.97753600 & -0.75657600 \\ -1.56117000 & 2.53116100 & -1.62199300 \\ -3.35243200 & 1.77445100 & -2.56338300 \\ -0.45336900 & -0.32783800 & 2.85922100 \\ -0.24997700 & -2.20303600 & 1.75806800 \\ -1.81703600 & -1.96731400 & 3.23217700 \\ -5.44230600 & -2.74580300 & -0.87738000 \\ -6.56228200 & -0.96432600 & -0.32884800 \\ -5.91553300 & -2.36993300 & 1.21139200 \\ -1.26629900 & -1.74367700 & -1.68551800 \\ -0.57496100 & 0.22451900 & -2.30873000 \\ 0.25108000 & -1.55227000 & -3.22693900 \\ 1.42177900 & 1.31630100 & 2.64252600 \\ 3.51968900 & 0.87903100 & 2.33007000 \\ 2.25181800 & -0.55475500 & 3.35037100 \\ 4.17458500 & -3.53570700 & -1.80363900 \\ 3.52953100 & -4.49435800 & 0.03739700 \\ 5.11719100 & -3.00825400 & 0.09338200 \\ 0.97449900 & 2.72563500 & -2.71443800 \\ 0.71243400 & 2.48309000 & -3.73986900 \\ 0.44806800 & 3.54951700 & -2.24860100 \\ 1.92087200 & 2.02244800 & -2.04760700 \\ 2.75528900 & 1.04849900 & -2.74436200 \\ 2.36244000 & 0.58578100 & -3.64622800 \\ 4.01381600 & 0.79179100 & -2.30949300 \\ 4.65383400 & 0.09686200 & -2.84644700 \\ 4.52070300 & 1.44922100 & -1.13085400 \\ 5.54396800 & 1.26249800 & -0.81808600\end{array}$




$\begin{array}{cccc}\mathrm{C} & 3.70652400 & 2.22376300 & -0.35349400 \\ \mathrm{H} & 4.07707000 & 2.58306900 & 0.59970200 \\ \mathrm{C} & 2.31026400 & 2.37422000 & -0.66470500 \\ \mathrm{C} & 1.41785000 & 2.84109900 & 0.31741700 \\ \mathrm{C} & 1.79442300 & 3.88155400 & 1.22552300 \\ \mathrm{H} & 2.82370900 & 4.22090600 & 1.19816300 \\ \mathrm{C} & 0.87539700 & 4.54807500 & 1.97830500 \\ \mathrm{H} & 1.16991300 & 5.39031000 & 2.59589900 \\ \mathrm{C} & -0.50654600 & 4.17746700 & 1.87280300 \\ \mathrm{H} & -1.27925500 & 4.74757400 & 2.37663300 \\ \mathrm{C} & -0.85461400 & 3.09786200 & 1.14130400 \\ \mathrm{H} & -1.87857000 & 2.76277300 & 1.07552800 \\ \mathrm{C} & -1.73095200 & 0.33354800 & 0.36771700 \\ \mathrm{C} & -2.77441800 & 0.76048200 & -0.49770700 \\ \mathrm{C} & -4.00210000 & 0.09571200 & -0.57773100 \\ \mathrm{H} & -4.76170900 & 0.45474900 & -1.26134300 \\ \mathrm{C} & -4.26088000 & -1.01575200 & 0.20578200 \\ \mathrm{C} & -3.29850600 & -1.43435000 & 1.11313200 \\ \mathrm{H} & -3.50791300 & -2.26838500 & 1.77039400 \\ \mathrm{C} & -2.07484400 & -0.77369600 & 1.19469900 \\ \mathrm{C} & -1.14294100 & -1.31940300 & 2.25699900 \\ \mathrm{C} & -5.55278200 & -1.77478500 & 0.05503600 \\ \mathrm{C} & -2.76565200 & 2.00551900 & -1.36814600 \\ \mathrm{C} & 0.95589900 & -0.19270300 & 0.10391200 \\ \mathrm{C} & 2.02330000 & -0.39881600 & 1.01380400 \\ \mathrm{C} & 2.98594100 & -1.39011700 & 0.80754500 \\ \mathrm{H} & 3.79894600 & -1.49796200 & 1.51569200 \\ \mathrm{C} & 2.91655300 & -2.23508600 & -0.28856400 \\ \mathrm{C} & 1.86669300 & -2.09244200 & -1.18509000 \\ \mathrm{H} & 1.81020100 & -2.73726100 & -2.05302800 \\ \mathrm{C} & 0.91445500 & -1.09583000 & -0.99272700 \\ \mathrm{C} & -0.16664500 & -1.03928800 & -2.04813300 \\ \mathrm{C} & 2.27502500 & 0.33224300 & 2.32642000 \\ \mathrm{C} & 3.93941100 & -3.31896100 & -0.49001800\end{array}$

$\begin{array}{lccc}\text { TS }_{\text {rot }} & & & \\ \mathrm{B} & -0.18358200 & 0.52784700 & 0.48698500 \\ \mathrm{~N} & -0.06186700 & 1.86603600 & 1.17701900 \\ \mathrm{~F} & -2.72397800 & 2.89670100 & 0.43057300 \\ \mathrm{~F} & -1.60867400 & 2.32095300 & -1.34658800 \\ \mathrm{~F} & -3.76537900 & 2.39567200 & -1.39838600 \\ \mathrm{~F} & -0.24960800 & -1.78777300 & 2.34689000 \\ \mathrm{~F} & -0.01175200 & -2.90344000 & 0.48519300 \\ \mathrm{~F} & -1.52577500 & -3.47339100 & 1.91559400 \\ \mathrm{~F} & -5.54229700 & -2.60638200 & -1.55619700 \\ \mathrm{~F} & -6.55457100 & -1.10722300 & -0.34790400 \\ \mathrm{~F} & -5.81842200 & -2.94496200 & 0.57293400 \\ \mathrm{~F} & -0.66371300 & -1.65633600 & -2.04980700 \\ \mathrm{~F} & -0.16233500 & 0.44433600 & -2.29856000 \\ \mathrm{~F} & 0.94015100 & -1.06654900 & -3.38989900 \\ \mathrm{~F} & 0.94034400 & 0.25874500 & 3.14006100\end{array}$

\begin{tabular}{|c|c|c|c|}
\hline $\mathrm{F}$ & 2.70776000 & 1.35320800 & 2.51555200 \\
\hline $\mathrm{F}$ & 2.89782200 & -0.60110100 & 3.43829300 \\
\hline$x^{2}$ & 5.08757500 & $0 \quad-2.58681900$ & $0 \quad-1.87220800$ \\
\hline $\mathrm{F}$ & 4.32151200 & $0 \quad-4.07829000$ & $0 \quad-0.49082700$ \\
\hline $\mathrm{F}$ & 5.65085000 & -2.51721300 & 0.23672100 \\
\hline $\mathrm{C}$ & 0.10998800 & 4.42783500 & $0 \quad-1.55162500$ \\
\hline $\mathrm{H}$ & -0.20582200 & $0 \quad 4.98753100$ & -2.42603100 \\
\hline $\mathrm{H}$ & -0.36052800 & 4.68296700 & -0.61037900 \\
\hline $\mathrm{C}$ & 1.03211900 & 3.41706800 & -1.67246500 \\
\hline $\mathrm{C}$ & 700 & 3.13467900 & 600 \\
\hline $\mathrm{H}$ & 1.17229000 & $3.58456800-$ & -3.83 \\
\hline $\mathrm{C}$ & 75668000 & 2.36766600 & -3.08 \\
\hline $\mathrm{H}$ & 18798500 & 2.18654600 & -4.06 \\
\hline $\mathrm{C}$ & 3.38155200 & 1.83369100 & 5500 \\
\hline $\mathrm{H}$ & 4.30683100 & 1.27203800 & -2.01029500 \\
\hline $\mathrm{C}$ & 800 & 2.000 & -0.6 \\
\hline $\mathrm{H}$ & 3.251 & 1.58196900 & 0.1 \\
\hline $\mathrm{C}$ & 1.50526800 & 2.58726900 & 6000 \\
\hline $\mathrm{C}$ & 0.90585800 & 2.77614800 & 0.78473700 \\
\hline $\mathrm{C}$ & 1.05279600 & 3.95480300 & 000 \\
\hline $\mathrm{H}$ & 1.78711200 & 4.66965400 & 1.1 \\
\hline $\mathrm{C}$ & 0.23863500 & 4.24342000 & 2.6 \\
\hline $\mathrm{H}$ & 2400 & 5.19038700 & 800 \\
\hline $\mathrm{C}$ & -0.73994500 & ) 3.31283500 & 1400 \\
\hline $\mathrm{H}$ & -1.40648900 & 3.49142100 & 1200 \\
\hline $\mathrm{C}$ & -0.86230600 & 2.14840400 & 49900 \\
\hline $\mathrm{H}$ & -1.59297100 & 1.38942200 & 2.49780700 \\
\hline $\mathrm{C}$ & -1.64342700 & -0.11441800 & 3800 \\
\hline $\mathrm{C}$ & -2.76543700 & 0.59388600 & 6600 \\
\hline $\mathrm{C}$ & -4.01168900 & -0.00290700 & 7900 \\
\hline $\mathrm{H}$ & -4.82691400 & 0.57317200 & 26200 \\
\hline $\mathrm{C}$ & -4.21692000 & -1.33441900 & -0.07447500 \\
\hline S & -3.16968900 & -2.06326900 & 0.47129700 \\
\hline $\mathrm{H}$ & -3.33678400 & -3.09301000 & 1600 \\
\hline $\mathrm{C}$ & -1.92390300 & -1.47536400 & 0.68 \\
\hline $\mathrm{C}$ & -0.91923500 & -2.40295700 & 51700 \\
\hline $\mathrm{C}$ & -5.53914900 & -1.99801300 & -0.34956300 \\
\hline $\mathrm{C}$ & -2.70672000 & 2.04588200 & -0.63273900 \\
\hline $\mathrm{C}$ & 1.12742500 & $\begin{array}{ll}0 & -0.29785700\end{array}$ & $\begin{array}{ll}0 & 0.20667300\end{array}$ \\
\hline$C$ & 2.13745600 & -0.52979000 & 1.18712200 \\
\hline $\mathrm{C}$ & 3.26256700 & -1.30871900 & 13900 \\
\hline $\mathrm{H}$ & 4.00882200 & -1.46565500 & 1.68404000 \\
\hline $\mathrm{C}$ & 3.44145100 & -1.89001000 & -0.33207400 \\
\hline $\mathrm{C}$ & 2.48556100 & -1.68158100 & -1.32059500 \\
\hline $\mathrm{H}$ & 2.62939000 & -2.10884100 & -2.30500400 \\
\hline C & 1.35787600 & -0.91450800 & -1.05517700 \\
\hline $\mathrm{C}$ & 0.37094500 & $\begin{array}{ll}0 & -0.78857100\end{array}$ & $\begin{array}{ll}0 & -2.19602500\end{array}$ \\
\hline 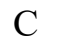 & 2.1517190 & $0.1065500 \mathrm{C}$ & 2.5631080 \\
\hline $\mathrm{C}$ & 4.6286240 & $0-2.7660320$ & -0.61191 \\
\hline
\end{tabular}

Int1b 


\begin{tabular}{|c|c|c|c|}
\hline B & -0.25349400 & 0.78372000 & 0.29796300 \\
\hline & 0.11341300 & 2.18254200 & \\
\hline & -3.22176000 & 3.07613300 & \\
\hline & -1.36786300 & 2.39129000 & 0 \\
\hline $\mathrm{F}$ & -3.24940100 & 1.83011800 & o -2.73653700 \\
\hline $\mathrm{F}$ & -0.88315100 & $0 \quad-0.0130850$ & 2.958638 \\
\hline $\mathrm{F}$ & -0.48184600 & -1.96405300 & 2.0541300 \\
\hline$F$ & -2.21348600 & -1.68893400 & 3.32271 \\
\hline $\mathrm{H}$ & -5.63672400 & -2.51495400 & $\begin{array}{ll}0 & -1.0\end{array}$ \\
\hline $\mathrm{F}$ & -6.67281800 & -0.66329200 & -0.57181500 \\
\hline $\mathrm{F}$ & -6.17949600 & -2.07819600 & 1.01572600 \\
\hline $\mathrm{F}$ & -1.28726100 & -1.99729900 & -1.37566700 \\
\hline $\mathrm{F}$ & -0.67928200 & -0.15183100 & -2.34836800 \\
\hline $\mathrm{F}$ & $0.13193400-$ & -2.04085400 & -3.01591500 \\
\hline $\mathrm{F}$ & 1.33010100 & 1.31463100 & 2.63775900 \\
\hline $\mathrm{F}$ & 3.31901800 & 0.48577300 & 2.81746300 \\
\hline $\mathrm{F}$ & 1.61396600 & -0.69232100 & 3.42875800 \\
\hline $\mathrm{F}$ & 4.35374600 & -3.41056300 & -1.74280900 \\
\hline $\mathrm{F}$ & 3.88023100 & -4.35280900 & 0.15825300 \\
\hline $\mathrm{F}$ & 5.30507500 & -2.71171100 & 0.09454100 \\
\hline $\mathrm{C}$ & 4.34927200 & 2.08910400 & 0.46187600 \\
\hline $\mathrm{H}$ & 5.42712400 & 1.96707900 & 0.50983000 \\
\hline $\mathrm{H}$ & 3.84038400 & 2.26383100 & 1.39856000 \\
\hline $\mathrm{C}$ & 3.69387700 & 1.96966600 & -0.73085600 \\
\hline $\mathrm{C}$ & 4.44730700 & 1.56863000 & -1.91013200 \\
\hline $\mathrm{H}$ & 5.53098900 & 1.55232800 & -1.82672700 \\
\hline $\mathrm{C}$ & 3.83588900 & 1.16800800 & -3.05483600 \\
\hline $\mathrm{H}$ & 4.42670400 & 0.84003200 & -3.90603700 \\
\hline $\mathrm{C}$ & 2.40680400 & 1.19505000 & -3.15344800 \\
\hline $\mathrm{H}$ & 1.92233300 & 0.92479600 & -4.08702100 \\
\hline $\mathrm{C}$ & 1.65139100 & 1.63403700 & -2.10146100 \\
\hline $\mathrm{H}$ & 0.58586600 & 1.72131100 & -2.22633600 \\
\hline $\mathrm{C}$ & 2.22880700 & $2.07875200-0$ & 0.87413800 \\
\hline $\mathrm{C}$ & 1.41757900 & 2.68148600 & 0.12877700 \\
\hline $\mathrm{C}$ & 1.82019700 & 3.87512100 & 0.79077300 \\
\hline $\mathrm{H}$ & 2.82614800 & 4.22733800 & 0.60228200 \\
\hline $\mathrm{C}$ & 0.94141500 & 4.63976700 & 1.50846000 \\
\hline $\mathrm{H}$ & 1.25270900 & 5.59121700 & 1.92704500 \\
\hline $\mathrm{C}$ & -0.41693700 & 4.21500300 & 1.61386000 \\
\hline $\mathrm{H}$ & -1.15715800 & 4.81865800 & 2.12725500 \\
\hline $\mathrm{C}$ & -0.79285400 & 3.03825700 & 1.06166600 \\
\hline $\mathrm{H}$ & -1.80279900 & 2.66426600 & 1.14283800 \\
\hline $\mathrm{C}$ & -1.81028100 & 0.34896900 & 0.29682800 \\
\hline $\mathrm{C}$ & -2.76765000 & 0.79523900 & -0.65301800 \\
\hline $\mathrm{C}$ & -4.02868900 & 0.20273800 & -0.76868400 \\
\hline $\mathrm{H}$ & -4.72459400 & 0.56247700 & -1.51644400 \\
\hline $\mathrm{C}$ & $.40437200-0$ & $.83900200 \quad 0$ & .06508500 \\
\hline $\mathrm{C}$ & -3.53750700 & -1.24444000 & 1.07065000 \\
\hline $\mathrm{H}$ & -3.84698500 & -2.00423100 & 1.77662600 \\
\hline $\mathrm{C}$ & -2.28171900 & -0.65537900 & 1.18443200 \\
\hline $\mathrm{C}$ & -1.45906900 & -1.09097800 & 2.37670000 \\
\hline
\end{tabular}

$\begin{array}{ccccc}\mathrm{C} & -5.73086300 & -1.52512900 & -0.13088000 \\ \mathrm{C} & -2.62458400 & 2.01246200 & -1.55333100 \\ \mathrm{C} & 0.88817500 & -0.32933800 & 0.18038000 \\ \mathrm{C} & 1.95842800 & -0.46821700 & 1.11001800 \\ \mathrm{C} & 3.00296600 & -1.36854400 & 0.89951000 \\ \mathrm{H} & 3.81025700 & -1.42909100 & 1.61849900 \\ \mathrm{C} & 3.02452300 & -2.18376200 & -0.22351600 \\ \mathrm{C} & 1.96014500 & -2.13916900 & -1.11199200 \\ \mathrm{H} & 1.95903500 & -2.79056300 & -1.97592800 \\ \mathrm{C} & 0.91142500 & -1.24382400 & -0.91135200 \\ \mathrm{C} & -0.22126400 & -1.35276900 & -1.91148500 \\ \mathrm{C} & 2.03758600 & 0.18058600 & 2.48558200 \\ \mathrm{C} & 4.14578800 & -3.16369100 & -0.43094500\end{array}$

T

B

$\mathrm{N}$

F

F

F

F

F

F

F

F

F

F

F

F

F

F

F

F

F

F

C

$\mathrm{H}$

$\mathrm{H}$

C

C

H

C

$\mathrm{H}$

C

$\mathrm{H}$

C

$\mathrm{H}$

C

C

C

H $\begin{array}{llll}-0.20060200 & 0.62685100 & 0.38780100\end{array}$

$\begin{array}{llll}-0.03491400 & 2.08328900 & 0.80980200\end{array}$ $\begin{array}{llll}-3.72196100 & 2.52393800 & -1.74275700\end{array}$ $\begin{array}{llll}-1.70589600 & 2.83721700 & -1.04376200\end{array}$ $\begin{array}{llll}-2.07220100 & 1.58131300 & -2.77805200\end{array}$ $\begin{array}{lll}-0.88891700 & -0.37193500 & 2.94856000\end{array}$ $\begin{array}{lll}-0.34938400 & -2.19143800 & 1.85331400\end{array}$ $\begin{array}{lll}-2.12091000 & -2.15091600 & 3.09181100\end{array}$ $\begin{array}{lll}-5.66976700 & -2.96856000 & 0.05025000\end{array}$ $\begin{array}{llll}-6.16822800 & -1.48801300 & -1.47508700\end{array}$ $\begin{array}{lll}-6.58303400 & -1.07970200 & 0.62061100\end{array}$ $\begin{array}{lll}0.36827000 & -2.25779200 & -2.84840300\end{array}$ $\begin{array}{llll}-1.03394400 & -2.16870900 & -1.20468000\end{array}$ $\begin{array}{lll}-0.50606600 & -0.35927600 & -2.29979200\end{array}$ $\begin{array}{llll}1.30700900 & 1.17847100 & 2.88720100\end{array}$ $\begin{array}{lll}3.33317000 & 0.45397100 & 3.11941100\end{array}$ $\begin{array}{lll}1.67695800 & -0.92679600 & 3.28481100\end{array}$ $\begin{array}{llll}5.37883400 & -1.78091600 & -1.56079300\end{array}$

$\begin{array}{llll}4.47489500 & -3.72154200 & -1.18535800\end{array}$ $\begin{array}{llll}5.52302500 & -2.67477500 & 0.41865300\end{array}$ $\begin{array}{llll}3.42915400 & 1.77093300 & 0.62923000\end{array}$

$\begin{array}{llll}4.44965300 & 1.46348700 & 0.83163500\end{array}$ $\begin{array}{llll}2.94726500 & 2.32489000 & 1.42482900\end{array}$ $\begin{array}{llll}2.99046100 & 1.88232000 & -0.70348800\end{array}$

$\begin{array}{llll}3.75375200 & 1.37127100 & -1.79238600\end{array}$

$\begin{array}{llll}4.76360900 & 1.01956300 & -1.60180200\end{array}$

$\begin{array}{llll}3.21503700 & 1.29358600 & -3.05989300\end{array}$

$\begin{array}{llll}3.81211900 & 0.89374200 & -3.87519500\end{array}$

$\begin{array}{llll}1.89605400 & 1.72286200 & -3.30578300\end{array}$

$\begin{array}{llll}1.48045400 & 1.66996700 & -4.30731600\end{array}$

$\begin{array}{llll}1.12407200 & 2.22432000 & -2.26764800\end{array}$

$\begin{array}{llll}0.11381800 & 2.55825700 & -2.45909500\end{array}$

$\begin{array}{llll}1.64720100 & 2.29068900 & -0.96743500\end{array}$

$\begin{array}{lll}0.87866200 & 2.86788600 & 0.16287700\end{array}$

$\begin{array}{llll}1.05273200 & 4.19209200 & 0.56768800\end{array}$

$\begin{array}{llll}1.80331500 & 4.78252700 & 0.05477700\end{array}$ 


\begin{tabular}{lccc}
$\mathrm{C}$ & 0.23766500 & 4.74530200 & 1.54895900 \\
$\mathrm{H}$ & 0.34266100 & 5.78837300 & 1.82995100 \\
$\mathrm{C}$ & -0.73847500 & 3.93845600 & 2.14936700 \\
$\mathrm{H}$ & -1.39883200 & 4.32493600 & 2.91761800 \\
$\mathrm{C}$ & -0.84606200 & 2.62143600 & 1.76681200 \\
$\mathrm{H}$ & -1.54546200 & 1.93507600 & 2.22417600 \\
$\mathrm{C}$ & -1.74521600 & 0.16411800 & 0.25190200 \\
$\mathrm{C}$ & -2.71481500 & 0.68276800 & -0.65014400 \\
$\mathrm{C}$ & -3.96377000 & 0.07524500 & -0.81891900 \\
$\mathrm{H}$ & -4.66102100 & 0.47023400 & -1.54688200 \\
$\mathrm{C}$ & -4.32601000 & -1.03056600 & -0.06582500 \\
$\mathrm{C}$ & -3.45880400 & -1.49485100 & 0.91330900 \\
$\mathrm{H}$ & -3.75573700 & -2.31744200 & 1.55123500 \\
$\mathrm{C}$ & -2.20986400 & -0.90148500 & 1.07197400 \\
$\mathrm{C}$ & -1.38075400 & -1.41817900 & 2.22992900 \\
$\mathrm{C}$ & -5.69024100 & -1.64739900 & -0.22318600 \\
$\mathrm{C}$ & -2.53591500 & 1.89606500 & -1.55168400 \\
$\mathrm{C}$ & 1.03148600 & -0.27633200 & 0.25112700 \\
$\mathrm{C}$ & 2.22167000 & -0.18907100 & 1.10395700 \\
$\mathrm{C}$ & 3.34894400 & -1.03093700 & 0.85117800 \\
$\mathrm{H}$ & 4.18947000 & -0.98496900 & 1.53212400 \\
$\mathrm{C}$ & 3.41622500 & -1.82238200 & -0.25588500 \\
$\mathrm{C}$ & 2.27969800 & -1.95856900 & -1.08798700 \\
$\mathrm{H}$ & 2.33239900 & -2.61805500 & -1.94286800 \\
$\mathrm{C}$ & 1.13386300 & -1.25421100 & -0.82101000 \\
$\mathrm{C}$ & 0.00068500 & -1.50777600 & -1.78706900 \\
$\mathrm{C}$ & 2.12456500 & 0.14151500 & 2.58457300 \\
$\mathrm{C}$ & 4.69416700 & -2.50555900 & -0.63524700 \\
& \multicolumn{4}{c}{$\mathrm{c}$}
\end{tabular}

$\begin{array}{cccc}\mathrm{H} & 3.85874400 & 1.61467200 & 1.38428500 \\ \mathrm{H} & 2.24886300 & 2.00510600 & 1.93318200 \\ \mathrm{C} & 2.56056100 & 2.32480400 & -0.15880600 \\ \mathrm{C} & 3.52695900 & 2.40537000 & -1.16563300 \\ \mathrm{H} & 4.46454400 & 1.87124800 & -1.04034500 \\ \mathrm{C} & 3.29809500 & 3.15864900 & -2.31586900 \\ \mathrm{H} & 4.05774600 & 3.20494700 & -3.09105800 \\ \mathrm{C} & 2.10115900 & 3.86398100 & -2.46487300 \\ \mathrm{H} & 1.92221400 & 4.46042100 & -3.35454300 \\ \mathrm{C} & 1.13111900 & 3.79465200 & -1.46945400 \\ \mathrm{H} & 0.18679300 & 4.31703800 & -1.58436800 \\ \mathrm{C} & 1.34396300 & 3.00212500 & -0.33324800 \\ \mathrm{C} & 0.33114500 & 2.97894100 & 0.74835400 \\ \mathrm{C} & -0.03433200 & 4.18400100 & 1.35472200 \\ \mathrm{H} & 0.42591900 & 5.09296500 & 0.98505900 \\ \mathrm{C} & -0.94237700 & 4.21520700 & 2.40460700 \\ \mathrm{H} & -1.22033100 & 5.15715400 & 2.86713000 \\ \mathrm{C} & -1.46597500 & 3.00617200 & 2.86481900 \\ \mathrm{H} & -2.15480500 & 2.96143400 & 3.70081400 \\ \mathrm{C} & -1.09459300 & 1.83737400 & 2.23362000 \\ \mathrm{H} & -1.46929700 & 0.87782800 & 2.55729800 \\ \mathrm{C} & -1.65539600 & -0.07629100 & 0.11874400 \\ \mathrm{C} & -2.57812800 & 0.62083100 & -0.70112500 \\ \mathrm{C} & -3.87634700 & 0.16617500 & -0.93360600 \\ \mathrm{H} & -4.53800800 & 0.71750700 & -1.58940100 \\ \mathrm{C} & -4.31827400 & -1.00781100 & -0.34068100 \\ \mathrm{C} & -3.46416600 & -1.70983600 & 0.49939100 \\ \mathrm{H} & -3.80994500 & -2.61985500 & 0.97150700 \\ \mathrm{C} & -2.16692900 & -1.25127800 & 0.72997200 \\ \mathrm{C} & -1.34556800 & -2.07362000 & 1.70865500 \\ \mathrm{C} & -5.73334400 & -1.47767400 & -0.54385500 \\ \mathrm{C} & -2.23481900 & 1.94312200 & -1.34842800 \\ \mathrm{C} & 1.13359100 & -0.23587900 & 0.09594900 \\ \mathrm{C} & 2.41130200 & 0.03635400 & 0.96735200 \\ \mathrm{C} & 3.66147700 & -0.68167500 & 0.50266000 \\ \mathrm{H} & 4.57693300 & -0.45398500 & 1.03626700 \\ \mathrm{C} & 3.66256200 & -1.57599900 & -0.49219100 \\ \mathrm{C} & 2.46938200 & -1.87141900 & -1.23684500 \\ \mathrm{H} & 2.54655700 & -2.55099800 & -2.07299000 \\ \mathrm{C} & 1.29695500 & -1.23258400 & -0.95851500 \\ \mathrm{C} & 0.19095300 & -1.55049600 & -1.95649000 \\ \mathrm{C} & 2.19077200 & -0.46415600 & 2.41712100 \\ \mathrm{C} & 4.92647400 & -2.26918400 & -0.91484000\end{array}$

\section{Part 2: Optimized structures for $\mathrm{BN}-4$}

$\begin{array}{lccc}\text { BN-4 } & & & \\ \mathrm{B} & -0.12092200 & 0.62726100 & -0.32362100 \\ \mathrm{~N} & 0.04345000 & 2.06990600 & 0.43441900 \\ \mathrm{C} & 0.07025100 & 1.00012900 & -1.90320400 \\ \mathrm{H} & -0.01592100 & 0.11079800 & -2.53060100\end{array}$




\begin{tabular}{cccc} 
H & -0.72414700 & 1.69026700 & -2.21357100 \\
$\mathrm{C}$ & 1.40678800 & 1.65224800 & -2.09870500 \\
$\mathrm{C}$ & 2.26712700 & 1.32376100 & -3.14977800 \\
$\mathrm{H}$ & 1.94356000 & 0.58295600 & -3.87612900 \\
$\mathrm{C}$ & 3.52378000 & 1.91672900 & -3.26974300 \\
$\mathrm{H}$ & 4.17757200 & 1.63758100 & -4.09169100 \\
$\mathrm{C}$ & 3.94847100 & 2.85423800 & -2.32530900 \\
$\mathrm{H}$ & 4.93458200 & 3.30297300 & -2.39900900 \\
$\mathrm{C}$ & 3.10634000 & 3.19720400 & -1.27256200 \\
$\mathrm{H}$ & 3.46026400 & 3.89154600 & -0.51653100 \\
$\mathrm{C}$ & 1.83469500 & 2.61029000 & -1.15269600 \\
$\mathrm{C}$ & 0.95519600 & 2.97491100 & -0.02645400 \\
$\mathrm{C}$ & 1.04353400 & 4.22829000 & 0.59812500 \\
$\mathrm{H}$ & 1.71968300 & 4.96883000 & 0.18959500 \\
$\mathrm{C}$ & 0.26816200 & 4.52573100 & 1.70890700 \\
$\mathrm{H}$ & 0.34604600 & 5.49812200 & 2.18598600 \\
$\mathrm{C}$ & -0.61342600 & 3.56009100 & 2.19479300 \\
$\mathrm{H}$ & -1.23600600 & 3.73537500 & 3.06459400 \\
$\mathrm{C}$ & -0.69651300 & 2.35482200 & 1.52248900 \\
$\mathrm{H}$ & -1.37261800 & 1.57236800 & 1.84069500 \\
$\mathrm{C}$ & -1.63427800 & 0.06200900 & -0.04931000 \\
$\mathrm{C}$ & -2.74551100 & 0.83123500 & -0.42122300 \\
$\mathrm{C}$ & -4.06463900 & 0.39992200 & -0.33252600 \\
$\mathrm{C}$ & -4.32744600 & -0.87694200 & 0.15660700 \\
$\mathrm{C}$ & -3.26594700 & -1.67905100 & 0.55797300 \\
$\mathrm{C}$ & -1.95771800 & -1.20002200 & 0.46002700 \\
$\mathrm{C}$ & 1.10055700 & -0.34971400 & 0.17129600 \\
$\mathrm{C}$ & 2.02196500 & -0.08634800 & 1.18411200 \\
$\mathrm{C}$ & 3.10467400 & -0.91508000 & 1.48173500 \\
$\mathrm{C}$ & 3.29512500 & -2.08176400 & 0.75289300 \\
$\mathrm{C}$ & 2.39300900 & -2.40269300 & -0.25884700 \\
$\mathrm{C}$ & 1.33387900 & -1.54241400 & -0.52130300 \\
$\mathrm{~F}$ & -2.56586500 & 2.09878300 & -0.86797900 \\
$\mathrm{~F}$ & -5.07715400 & 1.19774600 & -0.70203200 \\
$\mathrm{~F}$ & -5.58648600 & -1.31789400 & 0.25238100 \\
$\mathrm{~F}$ & -3.50605300 & -2.90243900 & 1.04945200 \\
$\mathrm{~F}$ & -1.00634100 & -2.04065500 & 0.90865600 \\
$\mathrm{~F}$ & 1.91836900 & 1.02418600 & 1.95812100 \\
$\mathrm{~F}$ & 3.95904200 & -0.59242800 & 2.46536900 \\
$\mathrm{~F}$ & 4.32583600 & -2.89214000 & 1.02389000 \\
$\mathrm{~F}$ & 2.55336600 & -3.53308500 & -0.96236800 \\
$\mathrm{~F}$ & 0.47783000 & -1.92787900 & -1.49454400 \\
& \multicolumn{3}{c}{}
\end{tabular}

$\mathrm{H}$

$\begin{array}{lccc}\text { BN-4: } & \left(\mathbf{S}_{\mathbf{1}} / \mathbf{S}_{\mathbf{1}}\right)_{\mathbf{X}} & & \\ \mathrm{B} & 0.34528200 & 0.56422400 & -0.12950900 \\ \mathrm{~N} & -0.07974400 & 1.96391100 & -0.39114800 \\ \mathrm{C} & -0.70653900 & 1.36551600 & 2.22840000 \\ \mathrm{H} & -0.43359700 & 0.86718900 & 3.15055000 \\ \mathrm{H} & 0.00113200 & 2.07049900 & 1.84322800 \\ \mathrm{C} & -1.99242600 & 1.20235100 & 1.70711500 \\ \mathrm{C} & -2.96010200 & 0.43979800 & 2.39855200 \\ \mathrm{H} & -2.61841100 & -0.12194700 & 3.26200800 \\ \mathrm{C} & -4.29437800 & 0.44228700 & 2.04067900 \\ \mathrm{H} & -5.02160900 & -0.13183700 & 2.60384900\end{array}$

$\begin{array}{lll}1.76499800 & 0.34525000 & -3.70786900\end{array}$ $\begin{array}{llll}3.44567000 & 1.57803200 & -3.19809100\end{array}$ $\begin{array}{llll}4.08656500 & 1.18586100 & -3.97994700\end{array}$ $\begin{array}{lll}3.91595800 & 2.57377000 & -2.32659800\end{array}$ $\begin{array}{llll}4.93127500 & 2.94321000 & -2.43192200\end{array}$ $\begin{array}{llll}3.12510800 & 3.05594800 & -1.29833000\end{array}$ $\begin{array}{llll}3.55328800 & 3.75766100 & -0.59163600\end{array}$ $\begin{array}{llll}1.81401100 & 2.59187900 & -1.10879600\end{array}$ $\begin{array}{lll}1.01587500 & 2.98007900 & 0.03416500\end{array}$ $\begin{array}{llll}1.19431700 & 4.21558200 & 0.67518500\end{array}$ $\begin{array}{lll}1.97333100 & 4.86951900 & 0.30119700\end{array}$ $\begin{array}{lll}0.38605600 & 4.63896400 & 1.69709100\end{array}$ $\begin{array}{lll}0.53253100 & 5.60574700 & 2.16226700\end{array}$ $\begin{array}{lll}-0.66894800 & 3.77679300 & 2.11068000\end{array}$ $\begin{array}{lll}-1.34392600 & 4.05763500 & 2.91026700\end{array}$ $\begin{array}{lll}-0.82843100 & 2.57568900 & 1.50723900\end{array}$ $\begin{array}{lll}-1.59692700 & 1.88503500 & 1.82444600\end{array}$ $\begin{array}{lll}-1.61799300 & 0.07405000 & 0.04945500\end{array}$ $\begin{array}{llll}-2.76292200 & 0.78369300 & -0.32915400\end{array}$ $\begin{array}{llll}-4.04011300 & 0.25042300 & -0.33877200\end{array}$ $\begin{array}{lll}-4.22618700 & -1.06733900 & 0.04775700\end{array}$ $\begin{array}{lll}-3.13108900 & -1.81760700 & 0.43978100\end{array}$ $\begin{array}{lll}-1.86622100 & -1.24666000 & 0.43384700\end{array}$ $\begin{array}{lll}1.10869400 & -0.24580900 & 0.20508100\end{array}$ $\begin{array}{lll}1.96219800 & -0.08979900 & 1.29197300\end{array}$ $\begin{array}{lll}3.05286300 & -0.91841000 & 1.52504700\end{array}$ $\begin{array}{lll}3.31985100 & -1.95795100 & 0.65285800\end{array}$ $2.48841000-2.16081400-0.43886100$ $\begin{array}{lll}1.41304800 & -1.31434300 & -0.63192100\end{array}$ $\begin{array}{lll}-2.66578100 & 2.06850000 & -0.71063700\end{array}$ $\begin{array}{llll}-5.08298600 & 0.98967900 & -0.71277100\end{array}$ $\begin{array}{lll}-5.44196100 & -1.60032600 & 0.04871500\end{array}$ $\begin{array}{lll}-3.29737900 & -3.08060000 & 0.82703700\end{array}$ $\begin{array}{lll}-0.87911800 & -2.04606500 & 0.85067900\end{array}$

$\begin{array}{lll}1.75779400 & 0.87970200 & 2.19363800\end{array}$ $\begin{array}{lll}3.83841500 & -0.71922300 & 2.58401500\end{array}$ $\begin{array}{lll}4.35993100 & -2.76096000 & 0.86033900\end{array}$ $\begin{array}{llll}2.72965400 & -3.16506200 & -1.28280800\end{array}$ $\begin{array}{llll}0.63164300 & -1.56801800 & -1.69817000\end{array}$

$\begin{array}{lccc}\mathbf{B N}-4 & \left(\mathbf{S}_{\mathbf{1}}\right) & & \\ \mathrm{B} & -0.15174600 & 0.72156600 & -0.03863100 \\ \mathrm{~N} & -0.00711600 & 2.11664900 & 0.49787200 \\ \mathrm{C} & -0.05833100 & 1.23720200 & -1.97234500 \\ \mathrm{H} & -0.36711500 & 0.40741500 & -2.59971700 \\ \mathrm{H} & -0.81661600 & 1.99820000 & -1.82659400 \\ \mathrm{C} & 1.30540800 & 1.64828200 & -2.06241700 \\ \mathrm{C} & 2.16017300 & 1.10672800 & -3.04226500\end{array}$




$\begin{array}{cccc}\text { C } & -4.70341400 & 1.18057100 & 0.94401200 \\ \text { H } & -5.74941400 & 1.20057200 & 0.66094700 \\ \text { C } & -3.74997300 & 1.85264400 & 0.18300900 \\ \text { H } & -4.06773500 & 2.35788300 & -0.72210800 \\ \text { C } & -2.39943400 & 1.83046800 & 0.49483300 \\ \text { C } & -1.40374200 & 2.42642600 & -0.39499100 \\ \text { C } & -1.70962000 & 3.49970200 & -1.22423800 \\ \text { H } & -2.75112200 & 3.77363700 & -1.33160100 \\ \text { C } & -0.73595700 & 4.27743200 & -1.81915200 \\ \text { H } & -0.99412000 & 5.12145100 & -2.44710700 \\ \text { C } & 0.58870200 & 3.99002400 & -1.52470900 \\ \text { H } & 1.38908100 & 4.64613100 & -1.84260000 \\ \text { C } & 0.87587500 & 2.86220400 & -0.80938500 \\ \text { H } & 1.88804500 & 2.56843100 & -0.57653000 \\ \text { C } & 1.88923900 & 0.22887900 & -0.05699300 \\ \text { C } & 2.79216600 & 0.73743200 & 0.87132900 \\ \text { C } & 4.11344900 & 0.34250800 & 0.93958400 \\ \text { C } & 4.58442500 & -0.60473400 & 0.05185200 \\ \text { C } & 3.73192800 & -1.13465200 & -0.89561500 \\ \text { C } & 2.41734300 & -0.71279700 & -0.93191700 \\ \text { C } & -0.61955800 & -0.66904300 & -0.19494900 \\ \text { C } & -1.57033400 & -0.81217500 & -1.19491500 \\ \text { C } & -2.38740400 & -1.91729500 & -1.31970200 \\ \text { C } & -2.25746100 & -2.95497300 & -0.41861700 \\ \text { C } & -1.31977100 & -2.86738800 & 0.59171000 \\ \text { C } & -0.52446600 & -1.74321800 & 0.68517800 \\ \text { F } & 2.40940200 & 1.67835100 & 1.74399000 \\ \text { F } & 4.93391200 & 0.86268500 & 1.84846500 \\ \text { F } & 5.85315500 & -0.99178500 & 0.10092300 \\ \text { F } & 4.18450300 & -2.03352100 & -1.76436400 \\ \text { F } & 1.63506100 & -1.24704700 & -1.87427600 \\ \text { F } & -1.71873500 & 0.16638600 & -2.09600000 \\ \text { F } & -3.28415500 & -1.99791500 & -2.30034100 \\ \text { F } & -3.03265700 & -4.02911300 & -0.51981000 \\ \text { F } & -1.20015400 & -3.85946500 & 1.47108700 \\ \text { F } & 0.35725200 & -1.71438700 & 1.68794100\end{array}$

$\begin{array}{lccc}\text { Int1 } & & & \\ \mathrm{B} & -0.15155100 & 0.86229600 & 0.38228900 \\ \mathrm{~N} & 0.12152300 & 2.23470100 & 0.68164000 \\ \mathrm{C} & 0.66699400 & 2.48047500 & -2.38009800 \\ \mathrm{H} & 0.40278200 & 2.19838300 & -3.39472000 \\ \mathrm{H} & -0.04479200 & 3.09490300 & -1.84282700 \\ \mathrm{C} & 1.83023400 & 2.05514000 & -1.82893400 \\ \mathrm{C} & 2.76813200 & 1.28887500 & -2.65054400 \\ \mathrm{H} & 2.38529000 & 0.85288600 & -3.56987900 \\ \mathrm{C} & 4.07448800 & 1.17305500 & -2.31859300 \\ \mathrm{H} & 4.76107600 & 0.62730800 & -2.96000800 \\ \mathrm{C} & 4.57251800 & 1.78349500 & -1.10747500 \\ \mathrm{H} & 5.62798300 & 1.69967300 & -0.86524400 \\ \mathrm{C} & 3.72518300 & 2.39988400 & -0.23999500\end{array}$

B

N

C

$\mathrm{H}$

$\mathrm{H}$

C

C

$\mathrm{H}$

C

$\mathrm{H}$

C

$\mathrm{H}$

C

$\mathrm{H}$

C

C $\begin{array}{llll}4.11839400 & 2.75286300 & 0.70720800\end{array}$

$\begin{array}{llll}2.29615400 & 2.44026700 & -0.46398100\end{array}$

$\begin{array}{lll}1.45380100 & 2.77266200 & 0.59604900\end{array}$

$\begin{array}{llll}1.82255700 & 3.65705800 & 1.66789300\end{array}$

$\begin{array}{llll}2.86234000 & 3.94224100 & 1.77389700\end{array}$

$\begin{array}{llll}0.87511100 & 4.26351700 & 2.43407000\end{array}$

$\begin{array}{llll}1.16064400 & 4.99043300 & 3.18786300\end{array}$

$\begin{array}{lll}-0.51907800 & 4.00351400 & 2.18055900\end{array}$

$\begin{array}{lll}-1.29298200 & 4.57944100 & 2.67576300\end{array}$

$\begin{array}{lll}-0.86125000 & 3.02694300 & 1.31633300\end{array}$

$\begin{array}{lll}-1.88748800 & 2.75506900 & 1.11649900\end{array}$

$\begin{array}{llll}-1.63551300 & 0.33770100 & 0.26961000\end{array}$

$\begin{array}{llll}-2.58572900 & 0.91544300 & -0.57594700\end{array}$

$\begin{array}{llll}-3.85502800 & 0.37841400 & -0.76597600\end{array}$

$\begin{array}{llll}-4.20725000 & -0.78724700 & -0.08738400\end{array}$

$\begin{array}{lll}-3.29249800 & -1.39635400 & 0.76995100\end{array}$

$\begin{array}{llll}-2.03053300 & -0.83099100 & 0.92716000\end{array}$

$\begin{array}{lll}0.99197100 & -0.19544200 & 0.15523000\end{array}$

$2.05743300 \quad-0.38054100 \quad 1.03772700$

$\begin{array}{llll}3.05512800 & -1.32487800 & 0.82280100\end{array}$

$2.99292200 \quad-2.13696000 \quad-0.30800800$

$\begin{array}{llll}1.94090800 & -1.99213700 & -1.20895500\end{array}$

$\begin{array}{llll}0.96146000 & -1.03807000 & -0.95782300\end{array}$

$\begin{array}{llll}-2.28804500 & 2.04808500 & -1.24389100\end{array}$

$\begin{array}{llll}-4.73311600 & 0.96382000 & -1.58807600\end{array}$

$\begin{array}{llll}-5.42167700 & -1.31453600 & -0.25302100\end{array}$

$\begin{array}{lll}-3.63416000 & -2.50907400 & 1.42862000\end{array}$

$\begin{array}{llll}-1.17522700 & -1.44541200 & 1.76316600\end{array}$

$\begin{array}{llll}2.15677700 & 0.38687400 & 2.13779800\end{array}$

$\begin{array}{rrr}4.06687500 & -1.46280700 & 1.68873000\end{array}$

$3.94634400 \quad-3.04572200 \quad-0.53166200$

$\begin{array}{llll}1.89065100 & -2.75618500 & -2.30747200\end{array}$

$\begin{array}{lll}-0.01734200 & -0.89762400 & -1.87361100\end{array}$

$\begin{array}{llll}-0.32581400 & 0.23298000 & -0.47061300\end{array}$

$\begin{array}{llll}-0.21509600 & 1.63343000 & -1.07697400\end{array}$

$\begin{array}{llll}3.27250700 & 1.42913200 & -0.08126800\end{array}$

$\begin{array}{llll}4.29257700 & 1.08649700 & 0.06797300\end{array}$

$3.08330600 \quad 1.92286300 \quad-1.02599500$

$\begin{array}{llll}2.46989400 & 1.68727300 & 1.04679000\end{array}$

$\begin{array}{lll}2.89395000 & 1.36148200 & 2.37116600\end{array}$

$\begin{array}{llll}3.92302300 & 1.04288700 & 2.51678600\end{array}$

$\begin{array}{lll}2.03674800 & 1.45067000 & 3.44520900\end{array}$

$\begin{array}{llll}2.39615600 & 1.20553100 & 4.44141400\end{array}$

$\begin{array}{llll}0.69629500 & 1.86386900 & 3.27060200\end{array}$

$\begin{array}{lll}0.03057900 & 1.94271000 & 4.12450200\end{array}$

$\begin{array}{lll}0.23941900 & 2.17448700 & 2.00303700\end{array}$

$\begin{array}{lll}-0.78816500 & 2.48686300 & 1.84887700\end{array}$

$\begin{array}{llll}1.10440300 & 2.09273000 & 0.89073700\end{array}$

$\begin{array}{llll}0.61951200 & 2.50408400 & -0.44235100\end{array}$ 


$\begin{array}{cccc}\text { C } & 0.92204100 & 3.72647800 & -1.04639400 \\ \text { H } & 1.61583600 & 4.38922400 & -0.54166000 \\ \text { C } & 0.28334400 & 4.09638600 & -2.22357100 \\ \text { H } & 0.48049200 & 5.06319900 & -2.67590400 \\ \text { C } & -0.65236900 & 3.22092900 & -2.79983600 \\ \text { H } & -1.18679500 & 3.48262500 & -3.70608800 \\ \text { C } & -0.87424000 & 1.99754700 & -2.20918600 \\ \text { H } & -1.55403500 & 1.25759400 & -2.61470000 \\ \text { C } & -1.79237800 & -0.24587700 & -0.19461700 \\ \text { C } & -2.19859300 & -1.58064800 & -0.36315700 \\ \text { C } & -3.49265900 & -2.01987000 & -0.10213200 \\ \text { C } & -4.45366700 & -1.10896700 & 0.33172700 \\ \text { C } & -4.10819600 & 0.23090000 & 0.49433400 \\ \text { C } & -2.80504500 & 0.63208700 & 0.22394200 \\ \text { C } & 0.93346100 & -0.59302100 & -0.33474000 \\ \text { C } & 1.03074100 & -1.67116000 & 0.58701800 \\ \text { C } & 2.12503300 & -2.49241100 & 0.67058900 \\ \text { C } & 3.24091600 & -2.26711700 & -0.17566800 \\ \text { C } & 3.20697600 & -1.24901700 & -1.08744900 \\ \text { C } & 2.12297200 & -0.32817000 & -1.08625800 \\ \text { F } & -1.33769900 & -2.49852600 & -0.82481500 \\ \text { F } & -3.82925400 & -3.30185300 & -0.28482400 \\ \text { F } & -5.70035000 & -1.51492400 & 0.58164600 \\ \text { F } & -5.02755200 & 1.11422500 & 0.90449200 \\ \text { F } & -2.53876400 & 1.94795100 & 0.39211300 \\ \text { F } & 0.00337700 & -1.91785300 & 1.42484800 \\ \text { F } & 2.19379800 & -3.48131500 & 1.57531800 \\ \text { F } & 4.31796800 & -3.05518500 & -0.05212600 \\ \text { F } & 4.26591400 & -0.99329100 & -1.87310100 \\ \text { F } & 1.97091600 & 0.38567600 & -2.24614500\end{array}$

$\begin{array}{lccc}\text { BN-4a } & & & \\ \mathrm{B} & -0.15050100 & 0.33160200 & -0.57214600 \\ \mathrm{~N} & -0.79961100 & 1.74029400 & -0.31454900 \\ \mathrm{C} & 1.90306200 & 2.21178000 & 0.84689500 \\ \mathrm{H} & 2.80025300 & 2.76052200 & 1.13849500 \\ \mathrm{H} & 1.30792500 & 2.89539200 & 0.24101700 \\ \mathrm{C} & 1.15416700 & 1.73788200 & 2.06317000 \\ \mathrm{C} & 1.83978400 & 1.27795200 & 3.19250900 \\ \mathrm{H} & 2.92603600 & 1.28341500 & 3.17971600 \\ \mathrm{C} & 1.14723500 & 0.81549300 & 4.31040800 \\ \mathrm{H} & 1.69843300 & 0.45640000 & 5.17499000 \\ \mathrm{C} & -0.25053500 & 0.81233300 & 4.32224000 \\ \mathrm{H} & -0.79147800 & 0.44602400 & 5.18967400 \\ \mathrm{C} & -0.95023800 & 1.27632400 & 3.21207800 \\ \mathrm{H} & -2.03611500 & 1.26141700 & 3.19891400 \\ \mathrm{C} & -0.25334600 & 1.72456000 & 2.08146400 \\ \mathrm{C} & -0.99448800 & 2.27039000 & 0.92760800 \\ \mathrm{C} & -1.85170700 & 3.36536600 & 1.08022000 \\ \mathrm{H} & -1.99506100 & 3.76260400 & 2.07850000 \\ \mathrm{C} & -2.46823900 & 3.94293400 & -0.02202900\end{array}$

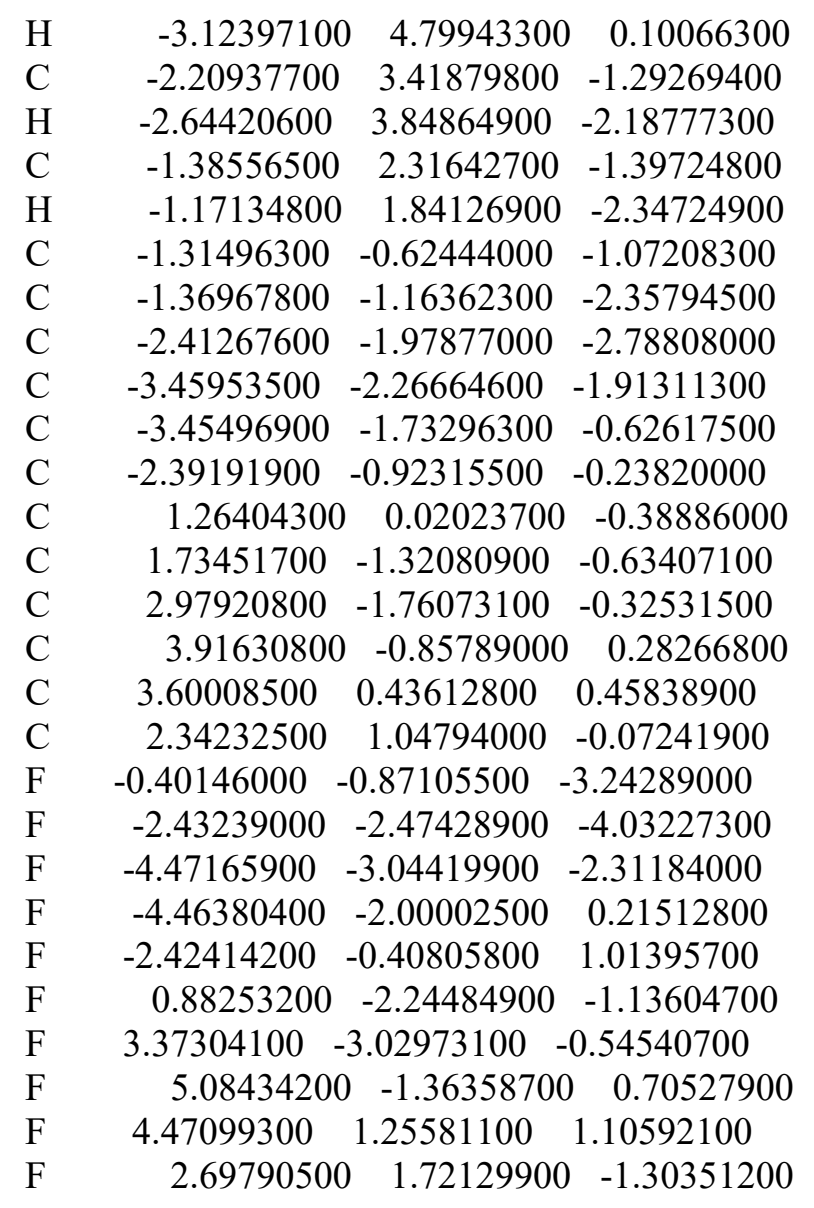

\begin{tabular}{lccc} 
TS $_{\mathbf{C B F}}$ & \multicolumn{4}{c}{} \\
$\mathrm{B}$ & -0.12065700 & 0.27003200 & -0.27417400 \\
$\mathrm{~N}$ & 0.31113500 & 1.66535000 & -0.83158300 \\
$\mathrm{C}$ & 2.81230100 & 0.16307800 & -1.33119600 \\
$\mathrm{H}$ & 3.72742000 & -0.23691100 & -1.77291600 \\
$\mathrm{H}$ & 2.42516300 & 0.90986400 & -2.02267700 \\
$\mathrm{C}$ & 3.11718600 & 0.75147700 & 0.02947900 \\
$\mathrm{C}$ & 4.15018500 & 0.24005900 & 0.82019000 \\
$\mathrm{H}$ & 4.76080700 & -0.56805900 & 0.42601400 \\
$\mathrm{C}$ & 4.39986700 & 0.74199000 & 2.09763000 \\
$\mathrm{H}$ & 5.20602000 & 0.32256600 & 2.69309200 \\
$\mathrm{C}$ & 3.61895500 & 1.77967400 & 2.61157300 \\
$\mathrm{H}$ & 3.80725100 & 2.16880700 & 3.60771400 \\
$\mathrm{C}$ & 2.59285100 & 2.31439900 & 1.83658800 \\
$\mathrm{H}$ & 1.97179300 & 3.11834500 & 2.22254100 \\
$\mathrm{C}$ & 2.33300200 & 1.79679500 & 0.56102900 \\
$\mathrm{C}$ & 1.30667800 & 2.42168800 & -0.29741000 \\
$\mathrm{C}$ & 1.40873000 & 3.77210000 & -0.65232800 \\
$\mathrm{H}$ & 2.20333700 & 4.35731700 & -0.20420500 \\
$\mathrm{C}$ & 0.54736900 & 4.32054000 & -1.59131600 \\
$\mathrm{H}$ & 0.63435200 & 5.36552500 & -1.87297600 \\
$\mathrm{C}$ & -0.40010300 & 3.49301700 & -2.20378200 \\
$\mathrm{H}$ & -1.05878900 & 3.85898800 & -2.98280600
\end{tabular}




$\begin{array}{cccc}\text { C } & -0.49167700 & 2.17847200 & -1.79913200 \\ \text { H } & -1.18808400 & 1.47736900 & -2.23646200 \\ \text { C } & -1.62707000 & 0.32821300 & 0.19154100 \\ \text { C } & -2.56801900 & -0.64919800 & -0.15141800 \\ \text { C } & -3.89466000 & -0.59092700 & 0.26893600 \\ \text { C } & -4.31729700 & 0.47303400 & 1.06351000 \\ \text { C } & -3.41288100 & 1.46734500 & 1.43109100 \\ \text { C } & -2.09847100 & 1.37675100 & 0.98754900 \\ \text { C } & 0.77800400 & -0.91377800 & -0.14480200 \\ \text { C } & 0.33639100 & -2.11128300 & 0.44916300 \\ \text { C } & 0.82995400 & -3.34771300 & 0.10041600 \\ \text { C } & 1.80908200 & -3.41234100 & -0.90882100 \\ \text { C } & 2.28897800 & -2.26985900 & -1.50786500 \\ \text { C } & 1.79198000 & -0.98007300 & -1.18838400 \\ \text { F } & -2.21507000 & -1.68469300 & -0.92096400 \\ \text { F } & -4.76833600 & -1.53871100 & -0.08811900 \\ \text { F } & -5.58658500 & 0.54057500 & 1.47222600 \\ \text { F } & -3.81320700 & 2.48790400 & 2.20118300 \\ \text { F } & -1.24896300 & 2.35672400 & 1.37427400 \\ \text { F } & -0.63080800 & -2.07450500 & 1.39099100 \\ \text { F } & 0.40528600 & -4.48030200 & 0.69138900 \\ \text { F } & 2.32660200 & -4.60554200 & -1.22381500 \\ \text { F } & 3.31037200 & -2.39389000 & -2.39260200 \\ \text { F } & 0.60271400 & -0.36660000 & -2.52020500 \\ & & & \end{array}$

\begin{tabular}{cccc} 
C & 0.89355400 & 3.39510400 & 4.06626500 \\
$\mathrm{H}$ & -0.12434800 & 3.46810900 & 4.43732700 \\
$\mathrm{C}$ & 1.60291800 & 4.54844800 & 3.75010300 \\
$\mathrm{H}$ & 1.13813400 & 5.52513300 & 3.85096300 \\
$\mathrm{C}$ & 2.90211600 & 4.45477800 & 3.22843800 \\
$\mathrm{C}$ & 3.62969500 & 5.68215700 & 2.84064100 \\
$\mathrm{C}$ & 3.88522000 & 6.67825800 & 3.78606500 \\
$\mathrm{H}$ & 3.49955000 & 6.54306700 & 4.79027800 \\
$\mathrm{C}$ & 4.64751400 & 7.79037800 & 3.44620300 \\
$\mathrm{H}$ & 4.85526700 & 8.56206300 & 4.18146600 \\
$\mathrm{C}$ & 5.15111600 & 7.88168300 & 2.15026800 \\
$\mathrm{H}$ & 5.76097800 & 8.72002000 & 1.83270300 \\
$\mathrm{C}$ & 4.83463500 & 6.88581000 & 1.23955400 \\
$\mathrm{H}$ & 5.14255100 & 6.93406800 & 0.20510600 \\
$\mathrm{C}$ & 1.97624800 & 4.66445100 & 0.42361400 \\
$\mathrm{C}$ & 1.17071000 & 5.79872800 & 0.54822200 \\
$\mathrm{C}$ & -0.20633800 & 5.74919700 & 0.73732700 \\
$\mathrm{C}$ & -0.83807200 & 4.50882900 & 0.78680400 \\
$\mathrm{C}$ & -0.08087100 & 3.35039700 & 0.65440300 \\
$\mathrm{C}$ & 1.29725900 & 3.44663900 & 0.46547100 \\
$\mathrm{C}$ & 4.45973400 & 3.47900400 & 0.10529500 \\
$\mathrm{C}$ & 4.56221000 & 2.96117200 & -1.18900600 \\
$\mathrm{C}$ & 5.24526000 & 1.78492300 & -1.49606000 \\
$\mathrm{C}$ & 5.85012300 & 1.06635800 & -0.47649000 \\
$\mathrm{C}$ & 5.74086000 & 1.54064100 & 0.82631900 \\
$\mathrm{C}$ & 5.06102600 & 2.71888300 & 1.13534700 \\
$\mathrm{~F}$ & 1.73682500 & 7.02738300 & 0.55439400 \\
$\mathrm{~F}$ & -0.92685800 & 6.87292100 & 0.87712000 \\
$\mathrm{~F}$ & -2.16123800 & 4.43410200 & 0.97640600 \\
$\mathrm{~F}$ & -0.67935600 & 2.15231500 & 0.72158000 \\
$\mathrm{~F}$ & 1.95877100 & 2.27644100 & 0.37663300 \\
$\mathrm{~F}$ & 3.95279500 & 3.55844200 & -2.23210800 \\
$\mathrm{~F}$ & 5.30796200 & 1.34033100 & -2.75863500 \\
$\mathrm{~F}$ & 6.51233700 & -0.06807000 & -0.73980800 \\
$\mathrm{~F}$ & 6.32043400 & 0.79855200 & 1.79974400 \\
$\mathrm{~F}$ & 3.85533600 & 5.69759400 & -0.83350900 \\
& & & \\
\hline
\end{tabular}


Table S6.1A. TD-DFT calculated electronic transitions for $\mathbf{B N - 0}$ along with their corresponding excitation energies and oscillator strengths.

\begin{tabular}{|c|c|c|c|c|}
\hline Compound & Spin State & Transition Configuration & $\begin{array}{c}\text { Excitation Energy } \\
(\mathrm{nm}, \mathrm{eV})\end{array}$ & $\begin{array}{l}\text { Oscillator } \\
\text { Strength }\end{array}$ \\
\hline \multirow{30}{*}{ BN-0 } & \multirow{4}{*}{$\mathrm{S}_{1}$} & HOMO-4 $\rightarrow$ LUMO (58\%) & \multirow{4}{*}{$306.26(4.05)$} & \multirow{4}{*}{0.0876} \\
\hline & & HOMO $\rightarrow$ LUMO (26\%) & & \\
\hline & & HOMO-3 $\rightarrow$ LUMO (2\%) & & \\
\hline & & HOMO-2 $\rightarrow$ LUMO (8\%) & & \\
\hline & \multirow{4}{*}{$\mathrm{S}_{2}$} & HOMO-4 $\rightarrow$ LUMO (16\%) & \multirow{4}{*}{$296.70(4.19)$} & \multirow{4}{*}{0.0842} \\
\hline & & HOMO $\rightarrow$ LUMO $(68 \%)$ & & \\
\hline & & HOMO-2 $\rightarrow$ LUMO (7\%) & & \\
\hline & & HOMO-1 $\rightarrow$ LUMO (4\%) & & \\
\hline & \multirow{4}{*}{$\mathrm{S}_{3}$} & HOMO-2 $\rightarrow$ LUMO (57\%) & \multirow{4}{*}{$271.22(4.57)$} & \multirow{4}{*}{0.0157} \\
\hline & & HOMO-1 $\rightarrow$ LUMO (26\%) & & \\
\hline & & HOMO-5 $\rightarrow$ LUMO (4\%) & & \\
\hline & & HOMO-4 $\rightarrow$ LUMO (5\%) & & \\
\hline & \multirow{6}{*}{$\mathrm{S}_{4}$} & HOMO-4 $\rightarrow$ LUMO (11\%) & \multirow{6}{*}{$264.91(4.68)$} & \multirow{6}{*}{0.0041} \\
\hline & & HOMO-2 $\rightarrow$ LUMO (21\%) & & \\
\hline & & HOMO-1 $\rightarrow$ LUMO (53\%) & & \\
\hline & & HOMO-5 $\rightarrow$ LUMO (4\%) & & \\
\hline & & HOMO-4 $\rightarrow$ LUMO+1 (2\%) & & \\
\hline & & HOMO $\rightarrow$ LUMO (3\%) & & \\
\hline & \multirow{4}{*}{$\mathrm{S}_{5}$} & HOMO-5 $\rightarrow$ LUMO (53\%) & \multirow{4}{*}{$262.24(4.73)$} & \multirow{4}{*}{0.0934} \\
\hline & & HOMO-4 $\rightarrow$ LUMO+1 (15\%) & & \\
\hline & & HOMO-1 $\rightarrow$ LUMO (14\%) & & \\
\hline & & HOMO-2 $\rightarrow$ LUMO+1 (4\%) & & \\
\hline & \multirow{4}{*}{$\mathrm{S}_{6}$} & HOMO-3 $\rightarrow$ LUMO (88\%) & \multirow{4}{*}{$249.95(4.96)$} & \multirow{4}{*}{0.0056} \\
\hline & & HOMO-4 $\rightarrow$ LUMO (3\%) & & \\
\hline & & HOMO-3 $\rightarrow$ LUMO+1 (3\%) & & \\
\hline & & $\mathrm{HOMO} \rightarrow \mathrm{LUMO}+(4 \%)$ & & \\
\hline & \multirow{4}{*}{$\mathrm{S}_{7}$} & $\mathrm{HOMO} \rightarrow \mathrm{LUMO}+1(82 \%)$ & \multirow{4}{*}{$248.59(4.99)$} & \multirow{4}{*}{0.0388} \\
\hline & & HOMO-5 $\rightarrow$ LUMO (3\%) & & \\
\hline & & HOMO-4 $\rightarrow$ LUMO+1 (2\%) & & \\
\hline & & HOMO-3 $\rightarrow$ LUMO (4\%) & & \\
\hline
\end{tabular}




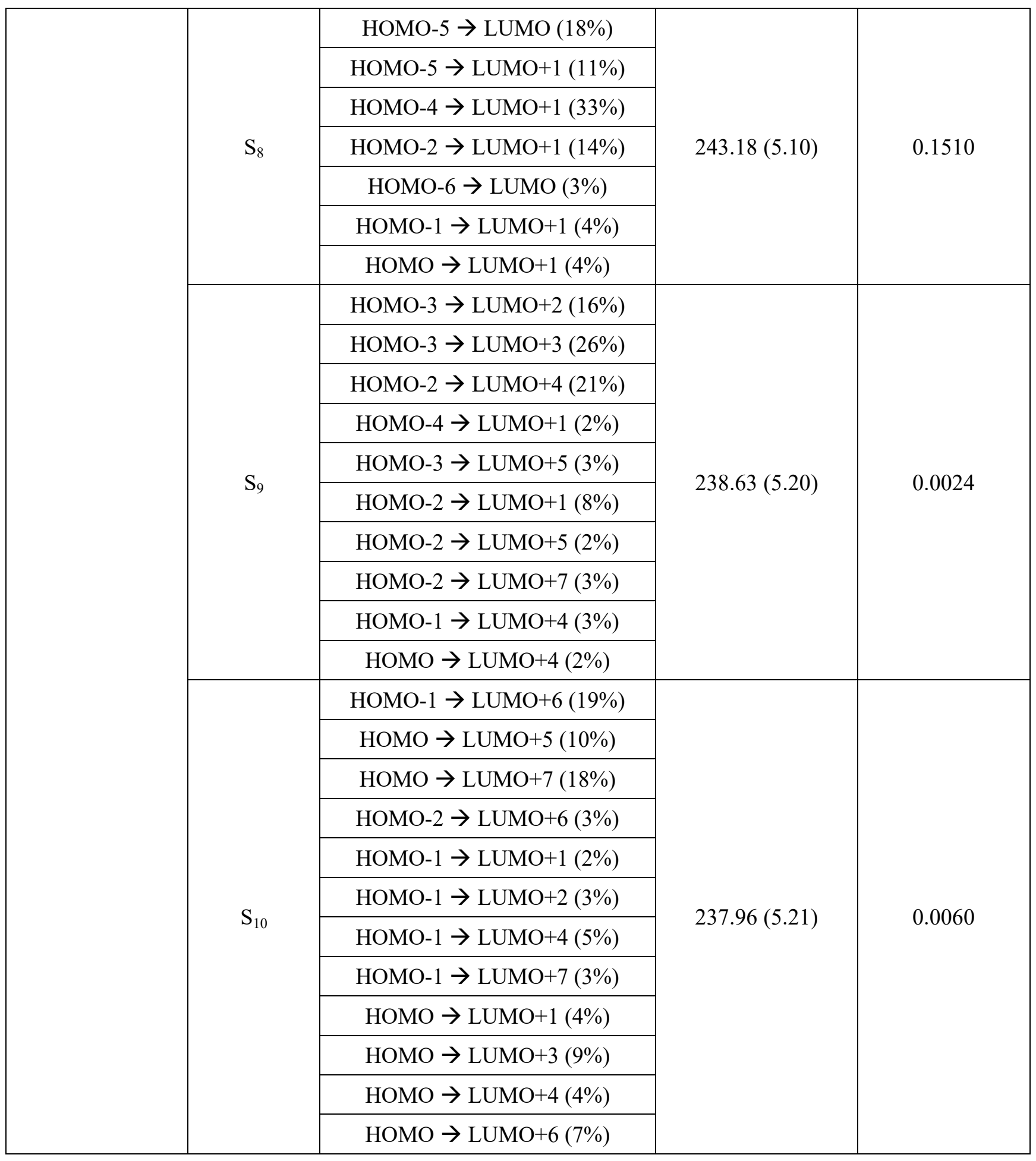


Table S6.1B. TD-DFT calculated electronic transitions for BN-phen-0 along with their corresponding excitation energies and oscillator strengths.

\begin{tabular}{|c|c|c|c|c|}
\hline Compound & Spin State & Transition Configuration & $\begin{array}{l}\text { Excitation Energy } \\
(\mathrm{nm}, \mathrm{eV})\end{array}$ & $\begin{array}{c}\text { Oscillator } \\
\text { Strength }\end{array}$ \\
\hline \multirow{28}{*}{ BN-phen-0 } & \multirow{2}{*}{$\mathrm{S}_{1}$} & HOMO $\rightarrow$ LUMO (92\%) & \multirow{2}{*}{$390.62(3.17)$} & \multirow{2}{*}{0.3113} \\
\hline & & $\mathrm{HOMO} \rightarrow \mathrm{LUMO}+1(5 \%)$ & & \\
\hline & \multirow{3}{*}{$\mathrm{S}_{2}$} & HOMO $\rightarrow$ LUMO+1 (90\%) & \multirow{3}{*}{$340.61(3.64)$} & \multirow{3}{*}{0.1026} \\
\hline & & HOMO-3 $\rightarrow$ LUMO $(2 \%)$ & & \\
\hline & & HOMO $\rightarrow$ LUMO $(6 \%)$ & & \\
\hline & \multirow{3}{*}{$\mathrm{S}_{3}$} & HOMO-3 $\rightarrow$ LUMO $(62 \%)$ & \multirow{3}{*}{$270.66(4.58)$} & \multirow{3}{*}{0.0209} \\
\hline & & HOMO $\rightarrow$ LUMO (34\%) & & \\
\hline & & HOMO $\rightarrow$ LUMO $(3 \%)$ & & \\
\hline & $\mathrm{S}_{4}$ & HOMO-1 $\rightarrow$ LUMO (95\%) & $251.31(4.93)$ & 0.0000 \\
\hline & \multirow{2}{*}{$\mathrm{S}_{5}$} & HOMO-3 $\rightarrow$ LUMO (31\%) & \multirow{2}{*}{$246.92(5.02)$} & \multirow{2}{*}{1.1298} \\
\hline & & HOMO $\rightarrow$ LUMO+3 (63\%) & & \\
\hline & \multirow{2}{*}{$\mathrm{S}_{6}$} & $\mathrm{HOMO} \rightarrow \mathrm{LUMO}+2(92 \%)$ & \multirow{2}{*}{$237.18(5.23)$} & \multirow{2}{*}{0.0001} \\
\hline & & HOMO-2 $\rightarrow$ LUMO+2 (3\%) & & \\
\hline & \multirow{4}{*}{$\mathrm{S}_{7}$} & $\mathrm{HOMO}-2 \rightarrow \mathrm{LUMO}+2(51 \%)$ & \multirow{4}{*}{$233.46(5.31)$} & \multirow{4}{*}{0.0019} \\
\hline & & HOMO-1 $\rightarrow$ LUMO+4 (42\%) & & \\
\hline & & HOMO-1 $\rightarrow$ LUMO+1 $(3 \%)$ & & \\
\hline & & $\mathrm{HOMO} \rightarrow \mathrm{LUMO}+2(4 \%)$ & & \\
\hline & $\mathrm{S}_{8}$ & HOMO-2 $\rightarrow$ LUMO $(95 \%)$ & $232.09(5.34)$ & 0.0046 \\
\hline & \multirow{3}{*}{$\mathrm{S}_{9}$} & $\mathrm{HOMO}-3 \rightarrow \mathrm{LUMO}+1(69 \%)$ & \multirow{3}{*}{$229.95(5.39)$} & \multirow{3}{*}{0.3228} \\
\hline & & HOMO $\rightarrow$ LUMO+5 (19\%) & & \\
\hline & & HOMO-2 $\rightarrow$ LUMO $(2 \%)$ & & \\
\hline & \multirow{7}{*}{$\mathrm{S}_{10}$} & HOMO-3 $\rightarrow$ LUMO+1 (20\%) & \multirow{7}{*}{$226.83(5.47)$} & \multirow{7}{*}{0.0396} \\
\hline & & $\mathrm{HOMO} \rightarrow \mathrm{LUMO}+5(53 \%)$ & & \\
\hline & & HOMO-6 $\rightarrow$ LUMO $(5 \%)$ & & \\
\hline & & HOMO-4 $\rightarrow$ LUMO (3\%) & & \\
\hline & & HOMO-4 $\rightarrow$ LUMO+1 (5\%) & & \\
\hline & & HOMO-3 $\rightarrow$ LUMO+3 (5\%) & & \\
\hline & & $\mathrm{HOMO} \rightarrow \mathrm{LUMO}+4(4 \%)$ & & \\
\hline
\end{tabular}


Table S6.1C. TD-DFT calculated electronic transitions for $\mathbf{B N}-1$ along with their corresponding excitation energies and oscillator strengths.

\begin{tabular}{|c|c|c|c|c|}
\hline Compound & Spin State & Transition Configuration & $\begin{array}{l}\text { Excitation Energy } \\
(\mathrm{nm}, \mathrm{eV})\end{array}$ & $\begin{array}{c}\text { Oscillator } \\
\text { Strength }\end{array}$ \\
\hline \multirow{30}{*}{ BN-1 } & \multirow{2}{*}{$\mathrm{S}_{1}$} & HOMO-4 $\rightarrow$ LUMO (25\%) & \multirow{2}{*}{$301.57(4.11)$} & \multirow{2}{*}{0.0679} \\
\hline & & HOMO $\rightarrow$ LUMO (70\%) & & \\
\hline & \multirow{4}{*}{$\mathrm{S}_{2}$} & HOMO-4 $\rightarrow$ LUMO (18\%) & \multirow{4}{*}{$287.52(4.31)$} & \multirow{4}{*}{0.0809} \\
\hline & & HOMO-1 $\rightarrow$ LUMO (63\%) & & \\
\hline & & HOMO-3 $\rightarrow$ LUMO $(3 \%)$ & & \\
\hline & & HOMO $\rightarrow$ LUMO (7\%) & & \\
\hline & \multirow{4}{*}{$\mathrm{S}_{3}$} & HOMO-4 $\rightarrow$ LUMO (47\%) & \multirow{4}{*}{$281.86(4.40)$} & \multirow{4}{*}{0.1697} \\
\hline & & HOMO-1 $\rightarrow$ LUMO (28\%) & & \\
\hline & & HOMO $\rightarrow$ LUMO (14\%) & & \\
\hline & & HOMO-2 $\rightarrow$ LUMO $(2 \%)$ & & \\
\hline & \multirow{2}{*}{$\mathrm{S}_{4}$} & HOMO-3 $\rightarrow$ LUMO (14\%) & \multirow{2}{*}{$271.23(4.57)$} & \multirow{2}{*}{0.0104} \\
\hline & & HOMO-2 $\rightarrow$ LUMO (79\%) & & \\
\hline & \multirow{5}{*}{$\mathrm{S}_{5}$} & HOMO-5 $\rightarrow$ LUMO (58\%) & \multirow{5}{*}{$261.30(4.74)$} & \multirow{5}{*}{0.0939} \\
\hline & & HOMO-4 $\rightarrow$ LUMO+1 (15\%) & & \\
\hline & & HOMO-6 $\rightarrow$ LUMO (2\%) & & \\
\hline & & HOMO-4 $\rightarrow$ LUMO+2 (5\%) & & \\
\hline & & $\mathrm{HOMO} \rightarrow \mathrm{LUMO}+1(8 \%)$ & & \\
\hline & \multirow{3}{*}{$\mathrm{S}_{6}$} & HOMO-3 $\rightarrow$ LUMO (78\%) & \multirow{3}{*}{$256.11(4.84)$} & \multirow{3}{*}{0.0029} \\
\hline & & HOMO-2 $\rightarrow$ LUMO (12\%) & & \\
\hline & & HOMO $\rightarrow$ LUMO (2\%) & & \\
\hline & \multirow{5}{*}{$\mathrm{S}_{7}$} & HOMO $\rightarrow$ LUMO+1 (69\%) & \multirow{5}{*}{$251.18(4.94)$} & \multirow{5}{*}{0.0133} \\
\hline & & HOMO-5 $\rightarrow$ LUMO $(6 \%)$ & & \\
\hline & & $\mathrm{HOMO}-3 \rightarrow \mathrm{LUMO}+4(3 \%)$ & & \\
\hline & & HOMO-1 $\rightarrow$ LUMO+1 (2\%) & & \\
\hline & & $\mathrm{HOMO} \rightarrow \mathrm{LUMO}+3(2 \%)$ & & \\
\hline & \multirow{5}{*}{$\mathrm{S}_{8}$} & HOMO-1 $\rightarrow$ LUMO+1 (61\%) & \multirow{5}{*}{$245.43(5.05)$} & \multirow{5}{*}{0.0066} \\
\hline & & HOMO-3 $\rightarrow$ LUMO+3 (3\%) & & \\
\hline & & $\mathrm{HOMO}-2 \rightarrow \mathrm{LUMO}+4(3 \%)$ & & \\
\hline & & HOMO-1 $\rightarrow$ LUMO+5 (6\%) & & \\
\hline & & $\mathrm{HOMO} \rightarrow \mathrm{LUMO}+3(6 \%)$ & & \\
\hline
\end{tabular}




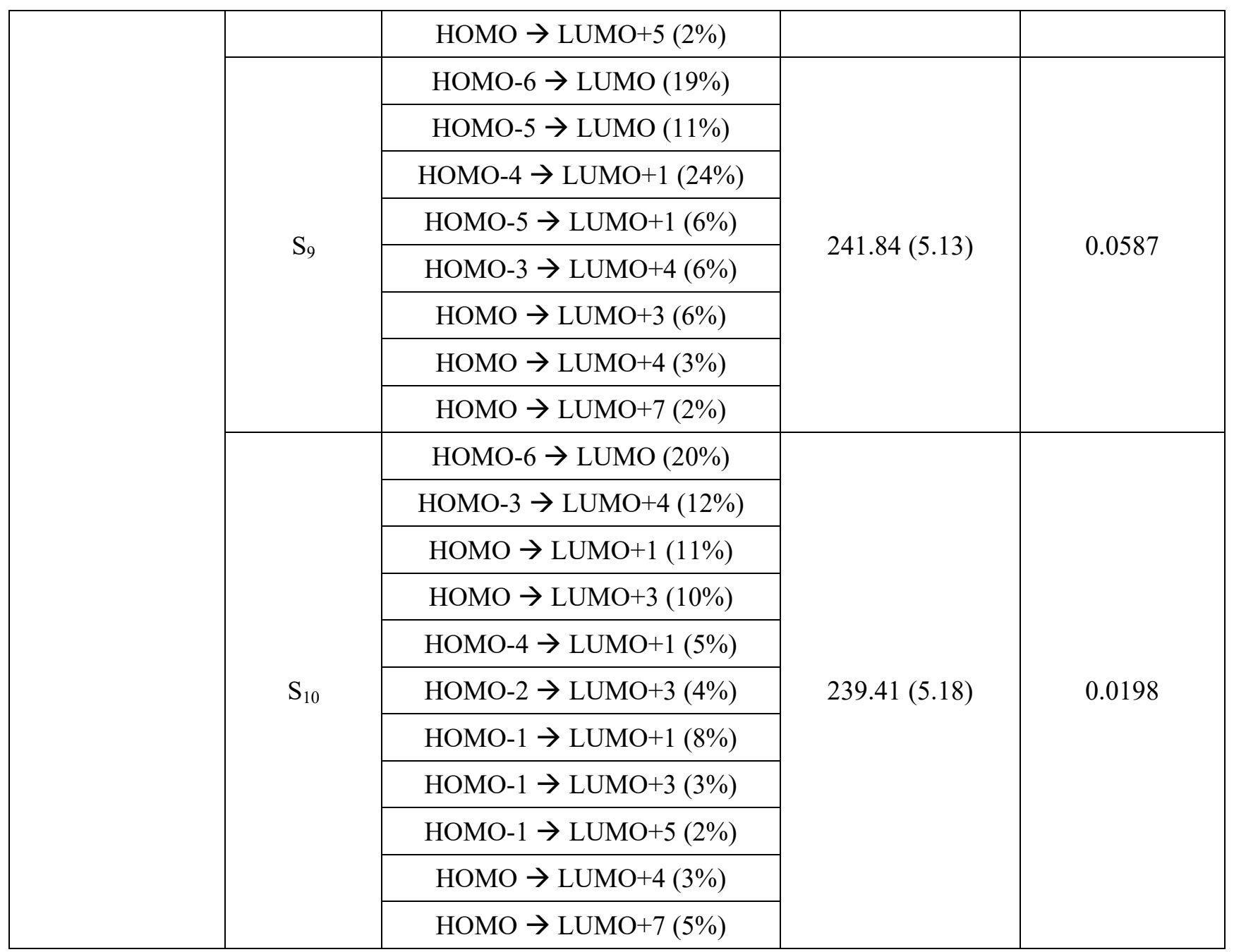

Table S6.1D. TD-DFT calculated electronic transitions for BN-phen-1 along with their corresponding excitation energies and oscillator strengths.

\begin{tabular}{|c|c|c|c|c|}
\hline Compound & Spin State & Transition Configuration & $\begin{array}{c}\text { Excitation Energy } \\
(\mathrm{nm}, \mathrm{eV})\end{array}$ & $\begin{array}{l}\text { Oscillator } \\
\text { Strength }\end{array}$ \\
\hline \multirow{10}{*}{ BN-phen-1 } & \multirow{2}{*}{$\mathrm{S}_{1}$} & HOMO $\rightarrow$ LUMO (91\%) & \multirow{2}{*}{$386.10(3.21)$} & \multirow{2}{*}{0.2932} \\
\hline & & $\mathrm{HOMO} \rightarrow \mathrm{LUMO}+1(5 \%)$ & & \\
\hline & \multirow{2}{*}{$\mathrm{S}_{2}$} & $\mathrm{HOMO} \rightarrow \mathrm{LUMO}+1(90 \%)$ & \multirow{2}{*}{$337.15(3.67)$} & \multirow{2}{*}{0.1110} \\
\hline & & HOMO $\rightarrow$ LUMO $(6 \%)$ & & \\
\hline & \multirow{4}{*}{$\mathrm{S}_{3}$} & HOMO-3 $\rightarrow$ LUMO (58\%) & \multirow{4}{*}{$268.07(4.63)$} & \multirow{4}{*}{0.0080} \\
\hline & & $\mathrm{HOMO} \rightarrow \mathrm{LUMO}+2(32 \%)$ & & \\
\hline & & HOMO $\rightarrow$ LUMO+1 (2\%) & & \\
\hline & & $\mathrm{HOMO} \rightarrow \mathrm{LUMO}+4(4 \%)$ & & \\
\hline & \multirow{2}{*}{$\mathrm{S}_{4}$} & HOMO-3 $\rightarrow$ LUMO $(32 \%)$ & \multirow{2}{*}{$245.87(5.04)$} & \multirow{2}{*}{1.0681} \\
\hline & & $\mathrm{HOMO} \rightarrow \mathrm{LUMO}+2(49 \%)$ & & \\
\hline
\end{tabular}




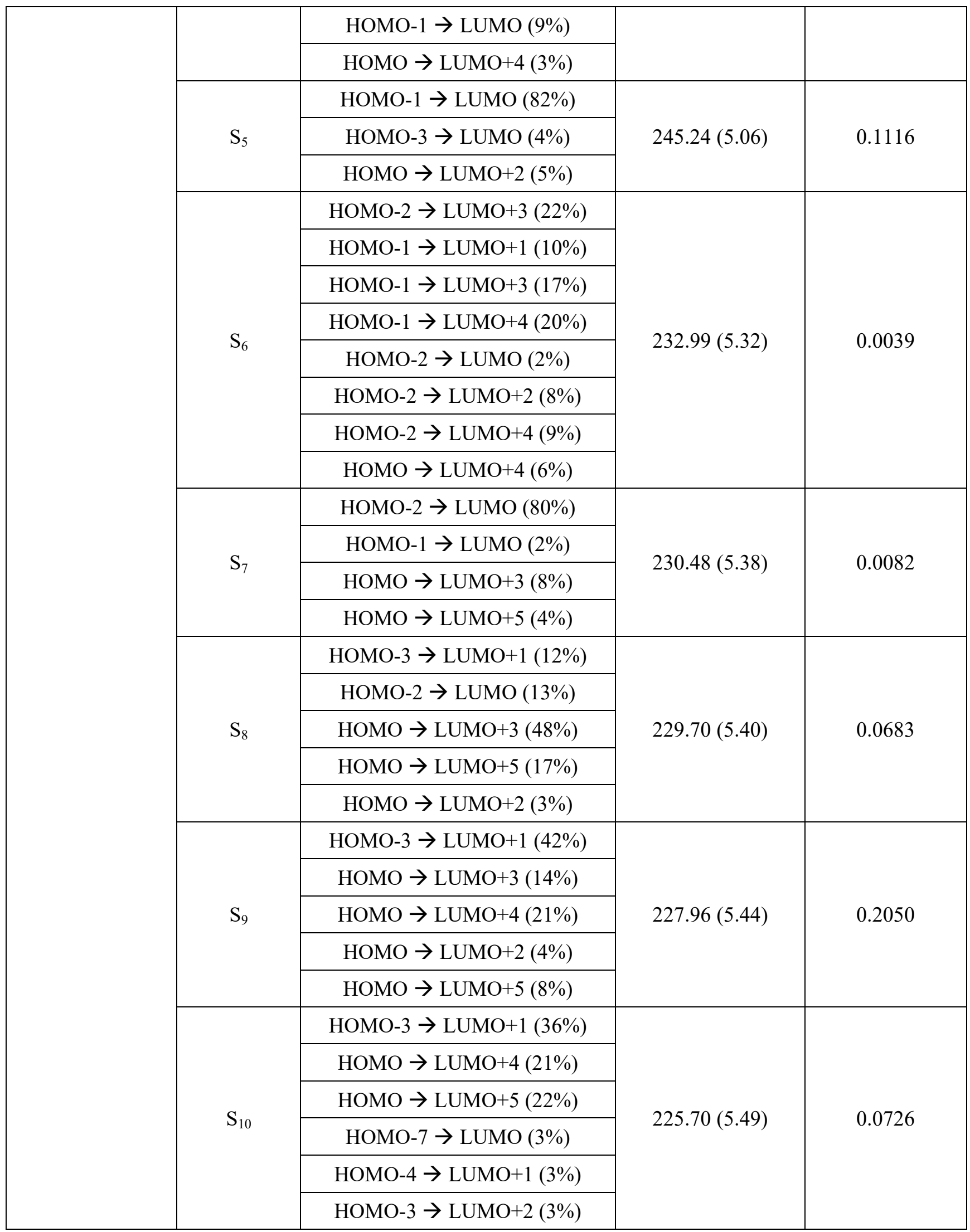


$\mathrm{HOMO} \rightarrow \mathrm{LUMO}+2$ (2\%)

Table S6.1E. TD-DFT calculated electronic transitions for BN-2 along with their corresponding excitation energies and oscillator strengths.

\begin{tabular}{|c|c|c|c|c|}
\hline Compound & Spin State & Transition Configuration & $\begin{array}{c}\text { Excitation Energy } \\
(\mathrm{nm}, \mathrm{eV})\end{array}$ & $\begin{array}{c}\text { Oscillator } \\
\text { Strength }\end{array}$ \\
\hline \multirow{29}{*}{ BN-2 } & $\mathrm{S}_{1}$ & HOMO $\rightarrow$ LUMO (23\%) & $293.61(4.22)$ & 0.1976 \\
\hline & \multirow{4}{*}{$\mathrm{S}_{2}$} & HOMO-1 $\rightarrow$ LUMO (69\%) & \multirow{4}{*}{$260.57(4.76)$} & \multirow{4}{*}{0.1014} \\
\hline & & $\mathrm{HOMO} \rightarrow \mathrm{LUMO}+1(8 \%)$ & & \\
\hline & & $\mathrm{HOMO} \rightarrow \mathrm{LUMO}+2(5 \%)$ & & \\
\hline & & $\mathrm{HOMO} \rightarrow \mathrm{LUMO}+6(4 \%)$ & & \\
\hline & \multirow{11}{*}{$\mathrm{S}_{3}$} & $\mathrm{HOMO}-2 \rightarrow \mathrm{LUMO}+5(18 \%)$ & \multirow{11}{*}{$236.65(5.24)$} & \multirow{11}{*}{0.0882} \\
\hline & & $\mathrm{HOMO} \rightarrow \mathrm{LUMO}+1(12 \%)$ & & \\
\hline & & HOMO-4 $\rightarrow$ LUMO+1 (6\%) & & \\
\hline & & HOMO-4 $\rightarrow$ LUMO+3 (9\%) & & \\
\hline & & HOMO-4 $\rightarrow$ LUMO+4 (7\%) & & \\
\hline & & $\mathrm{HOMO}-4 \rightarrow \mathrm{LUMO}+5(2 \%)$ & & \\
\hline & & $\mathrm{HOMO}-3 \rightarrow \mathrm{LUMO}+5(4 \%)$ & & \\
\hline & & $\mathrm{HOMO}-2 \rightarrow \mathrm{LUMO}+4$ (5\%) & & \\
\hline & & HOMO-2 $\rightarrow$ LUMO+2 (9\%) & & \\
\hline & & HOMO-1 $\rightarrow$ LUMO (5\%) & & \\
\hline & & $\mathrm{HOMO} \rightarrow \mathrm{LUMO}+2(8 \%)$ & & \\
\hline & \multirow{13}{*}{$\mathrm{S}_{4}$} & HOMO $\rightarrow$ LUMO+1 (10\%) & \multirow{13}{*}{$235.85(5.26)$} & \multirow{13}{*}{0.0654} \\
\hline & & $\mathrm{HOMO} \rightarrow \mathrm{LUMO}+2(17 \%)$ & & \\
\hline & & HOMO-6 $\rightarrow$ LUMO (3\%) & & \\
\hline & & HOMO-5 $\rightarrow$ LUMO+1 (9\%) & & \\
\hline & & HOMO-5 $\rightarrow$ LUMO+2 (5\%) & & \\
\hline & & HOMO-5 $\rightarrow$ LUMO+3 (2\%) & & \\
\hline & & $\mathrm{HOMO}-4 \rightarrow \mathrm{LUMO}+1(3 \%)$ & & \\
\hline & & $\mathrm{HOMO}-4 \rightarrow \mathrm{LUMO}+4(5 \%)$ & & \\
\hline & & $\mathrm{HOMO}-3 \rightarrow \mathrm{LUMO}+3(7 \%)$ & & \\
\hline & & $\mathrm{HOMO}-3 \rightarrow \mathrm{LUMO}+4(4 \%)$ & & \\
\hline & & HOMO-2 $\rightarrow$ LUMO+2 (5\%) & & \\
\hline & & HOMO-2 $\rightarrow$ LUMO+5 (5\%) & & \\
\hline & & HOMO-1 $\rightarrow$ LUMO $(5 \%)$ & & \\
\hline
\end{tabular}




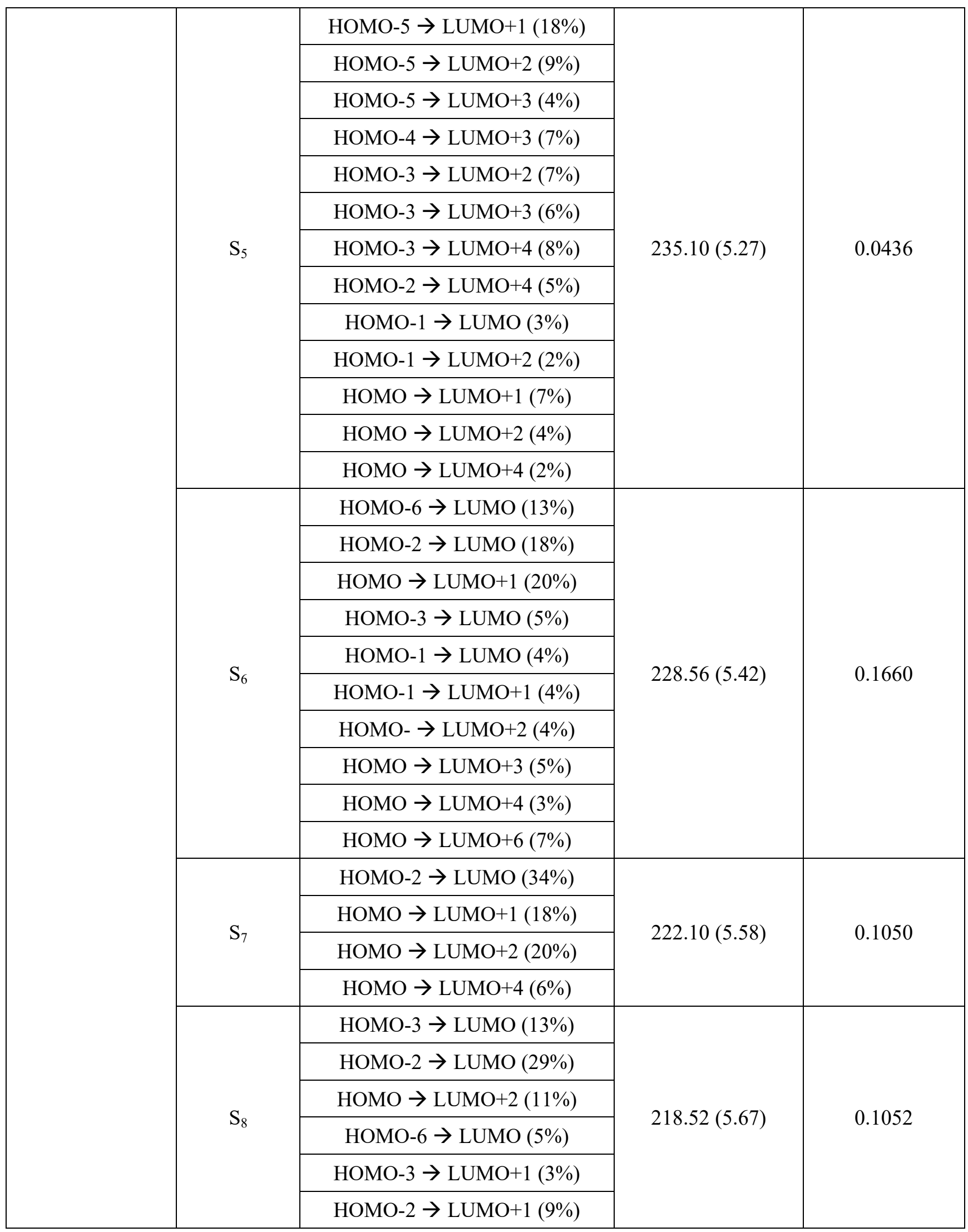




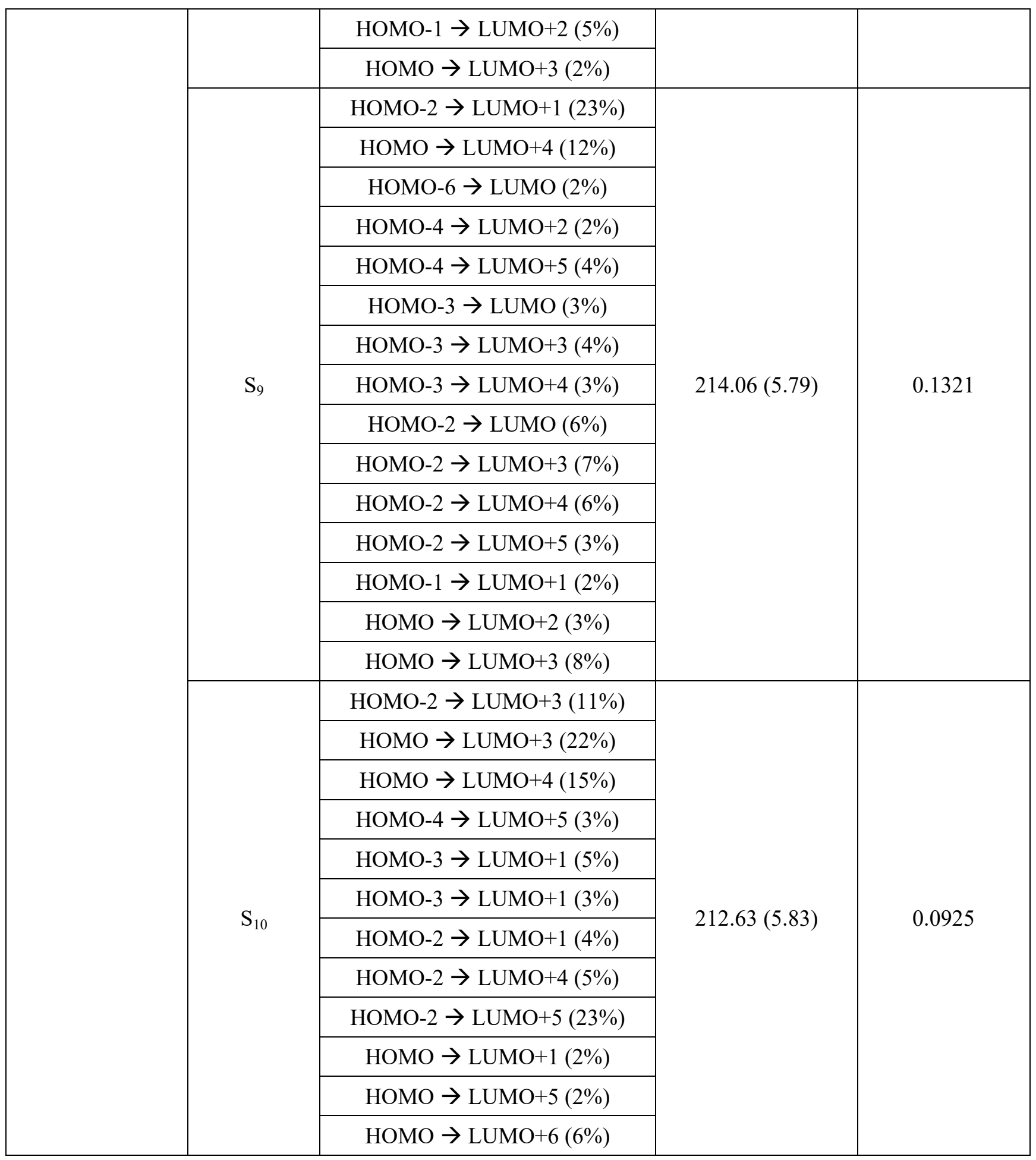

Table S6.1F. TD-DFT calculated electronic transitions for $\mathbf{B N - 2 a}$ along with their corresponding excitation energies and oscillator strengths. 


\begin{tabular}{|c|c|c|c|c|}
\hline Compound & Spin State & Transition Configuration & $\begin{array}{l}\text { Excitation Energy } \\
(\mathrm{nm}, \mathrm{eV})\end{array}$ & $\begin{array}{l}\text { Oscillator } \\
\text { Strength }\end{array}$ \\
\hline \multirow{32}{*}{ BN-2a } & $\mathrm{S}_{1}$ & HOMO $\rightarrow$ LUMO (97\%) & $461.96(2.68)$ & 0.1299 \\
\hline & \multirow{4}{*}{$\mathrm{S}_{2}$} & $\mathrm{HOMO} \rightarrow \mathrm{LUMO}+1(80 \%)$ & \multirow{4}{*}{$391.64(3.17)$} & \multirow{4}{*}{0.1879} \\
\hline & & $\mathrm{HOMO} \rightarrow \mathrm{LUMO}+2(7 \%)$ & & \\
\hline & & $\mathrm{HOMO} \rightarrow \mathrm{LUMO}+3(4 \%)$ & & \\
\hline & & $\mathrm{HOMO} \rightarrow \mathrm{LUMO}+4(5 \%)$ & & \\
\hline & \multirow{4}{*}{$\mathrm{S}_{3}$} & $\mathrm{HOMO} \rightarrow \mathrm{LUMO}+1(16 \%)$ & \multirow{4}{*}{$342.09(3.62)$} & \multirow{4}{*}{0.0108} \\
\hline & & $\mathrm{HOMO} \rightarrow \mathrm{LUMO}+2(16 \%)$ & & \\
\hline & & $\mathrm{HOMO} \rightarrow \mathrm{LUMO}+3(41 \%)$ & & \\
\hline & & $\mathrm{HOMO} \rightarrow \mathrm{LUMO}+4(23 \%)$ & & \\
\hline & \multirow{3}{*}{$\mathrm{S}_{4}$} & $\mathrm{HOMO} \rightarrow \mathrm{LUMO}+2(71 \%)$ & \multirow{3}{*}{$316.35(3.92)$} & \multirow{3}{*}{0.0043} \\
\hline & & $\mathrm{HOMO} \rightarrow \mathrm{LUMO}+3(22 \%)$ & & \\
\hline & & $\mathrm{HOMO} \rightarrow \mathrm{LUMO}+4(4 \%)$ & & \\
\hline & \multirow{3}{*}{$\mathrm{S}_{5}$} & $\mathrm{HOMO} \rightarrow \mathrm{LUMO}+3(29 \%)$ & \multirow{3}{*}{$294.03(4.22)$} & \multirow{3}{*}{0.0029} \\
\hline & & $\mathrm{HOMO} \rightarrow \mathrm{LUMO}+4(67 \%)$ & & \\
\hline & & $\mathrm{HOMO} \rightarrow \mathrm{LUMO}+2(2 \%)$ & & \\
\hline & \multirow{4}{*}{$\mathrm{S}_{6}$} & HOMO-2 $\rightarrow$ LUMO (12\%) & \multirow{4}{*}{$271.65(4.56)$} & \multirow{4}{*}{0.2118} \\
\hline & & HOMO-1 $\rightarrow$ LUMO (73\%) & & \\
\hline & & HOMO-6 $\rightarrow$ LUMO $(3 \%)$ & & \\
\hline & & HOMO-3 $\rightarrow$ LUMO (4\%) & & \\
\hline & \multirow{3}{*}{$\mathrm{S}_{7}$} & $\mathrm{HOMO} \rightarrow \mathrm{LUMO}+5(89 \%)$ & \multirow{3}{*}{$254.13(4.88)$} & \multirow{3}{*}{0.0170} \\
\hline & & HOMO-2 $\rightarrow$ LUMO (4\%) & & \\
\hline & & $\mathrm{HOMO} \rightarrow \mathrm{LUMO}+6(3 \%)$ & & \\
\hline & \multirow{7}{*}{$\mathrm{S}_{8}$} & HOMO-2 $\rightarrow$ LUMO (58\%) & \multirow{7}{*}{$251.46(4.93)$} & \multirow{7}{*}{0.0315} \\
\hline & & HOMO-1 $\rightarrow$ LUMO (12\%) & & \\
\hline & & $\mathrm{HOMO} \rightarrow \mathrm{LUMO}+6(10 \%)$ & & \\
\hline & & HOMO-2 $\rightarrow$ LUMO+2 (3\%) & & \\
\hline & & HOMO-2 $\rightarrow$ LUMO+5 (2\%) & & \\
\hline & & HOMO-1 $\rightarrow$ LUMO+6 (2\%) & & \\
\hline & & $\mathrm{HOMO} \rightarrow \mathrm{LUMO}+5(3 \%)$ & & \\
\hline & \multirow{3}{*}{$\mathrm{S}_{9}$} & $\mathrm{HOMO} \rightarrow \mathrm{LUMO}+6(83 \%)$ & \multirow{3}{*}{$245.94(5.04)$} & \multirow{3}{*}{0.0236} \\
\hline & & HOMO-2 $\rightarrow$ LUMO (7\%) & & \\
\hline & & $\mathrm{HOMO} \rightarrow \mathrm{LUMO}+5(6 \%)$ & & \\
\hline
\end{tabular}




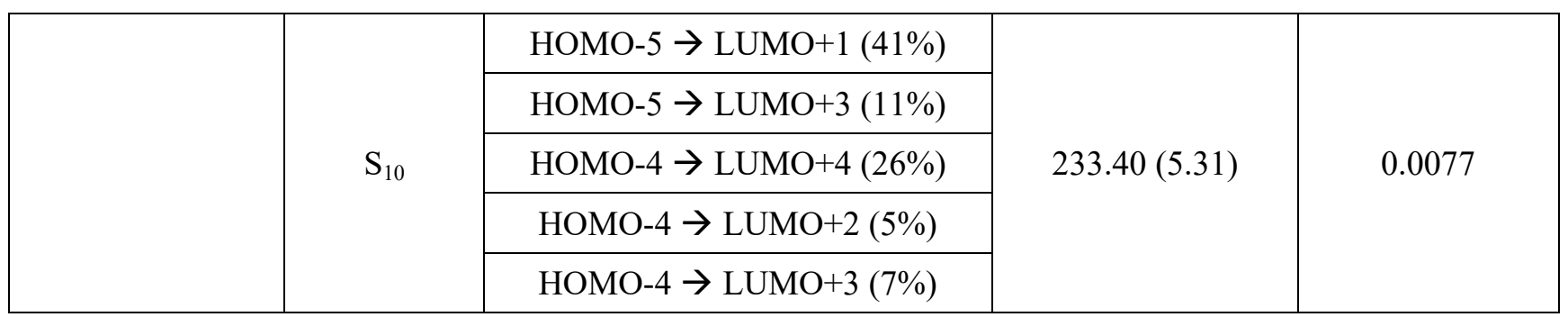

Table S6.1G. TD-DFT calculated electronic transitions for $\mathbf{B N}-3$ along with their corresponding excitation energies and oscillator strengths.

\begin{tabular}{|c|c|c|c|c|}
\hline Compound & Spin State & Transition Configuration & $\begin{array}{c}\text { Excitation Energy } \\
(\mathbf{n m}, \mathbf{e V})\end{array}$ & $\begin{array}{l}\text { Oscillator } \\
\text { Strength }\end{array}$ \\
\hline \multirow{25}{*}{ BN-3 } & $\mathrm{S}_{1}$ & HOMO $\rightarrow$ LUMO (95\%) & $311.07(3.99)$ & 0.3439 \\
\hline & \multirow{2}{*}{$\mathrm{S}_{2}$} & HOMO-2 $\rightarrow$ LUMO (18\%) & \multirow{2}{*}{$271.87(4.56)$} & \multirow{2}{*}{0.1649} \\
\hline & & HOMO-1 $\rightarrow$ LUMO (71\%) & & \\
\hline & \multirow{4}{*}{$\mathrm{S}_{3}$} & HOMO-2 $\rightarrow$ LUMO (65\%) & \multirow{4}{*}{$258.54(4.80)$} & \multirow{4}{*}{0.1422} \\
\hline & & HOMO-1 $\rightarrow$ LUMO (18\%) & & \\
\hline & & HOMO-4 $\rightarrow$ LUMO (3\%) & & \\
\hline & & $\mathrm{HOMO} \rightarrow \mathrm{LUMO}+6(2 \%)$ & & \\
\hline & \multirow{8}{*}{$\mathrm{S}_{4}$} & HOMO-5 $\rightarrow$ LUMO+3 (17\%) & \multirow{8}{*}{$237.11(5.23)$} & \multirow{8}{*}{0.0222} \\
\hline & & HOMO-3 $\rightarrow$ LUMO (15\%) & & \\
\hline & & HOMO-3 $\rightarrow$ LUMO+4 (27\%) & & \\
\hline & & HOMO-5 $\rightarrow$ LUMO+1 (5\%) & & \\
\hline & & HOMO-4 $\rightarrow$ LUMO+1 (2\%) & & \\
\hline & & HOMO-4 $\rightarrow$ LUMO+3 (4\%) & & \\
\hline & & HOMO-4 $\rightarrow$ LUMO+4 (4\%) & & \\
\hline & & $\mathrm{HOMO}-3 \rightarrow \mathrm{LUMO}+2(5 \%)$ & & \\
\hline & \multirow{9}{*}{$\mathrm{S}_{5}$} & HOMO-6 $\rightarrow$ LUMO+1 (36\%) & \multirow{9}{*}{$236.04(5.25)$} & \multirow{9}{*}{0.0000} \\
\hline & & HOMO-4 $\rightarrow$ LUMO+2 (17\%) & & \\
\hline & & HOMO-6 $\rightarrow$ LUMO+2 (2\%) & & \\
\hline & & HOMO-6 $\rightarrow$ LUMO+3 (4\%) & & \\
\hline & & HOMO-5 $\rightarrow$ LUMO+2 (9\%) & & \\
\hline & & HOMO-4 $\rightarrow$ LUMO+3 (7\%) & & \\
\hline & & HOMO-3 $\rightarrow$ LUMO+2 (4\%) & & \\
\hline & & HOMO-3 $\rightarrow$ LUMO+4 (5\%) & & \\
\hline & & HOMO-2 $\rightarrow$ LUMO+2 (4\%) & & \\
\hline & $\mathrm{S}_{6}$ & HOMO-3 $\rightarrow$ LUMO (62\%) & $232.26(5.34)$ & 0.0562 \\
\hline
\end{tabular}




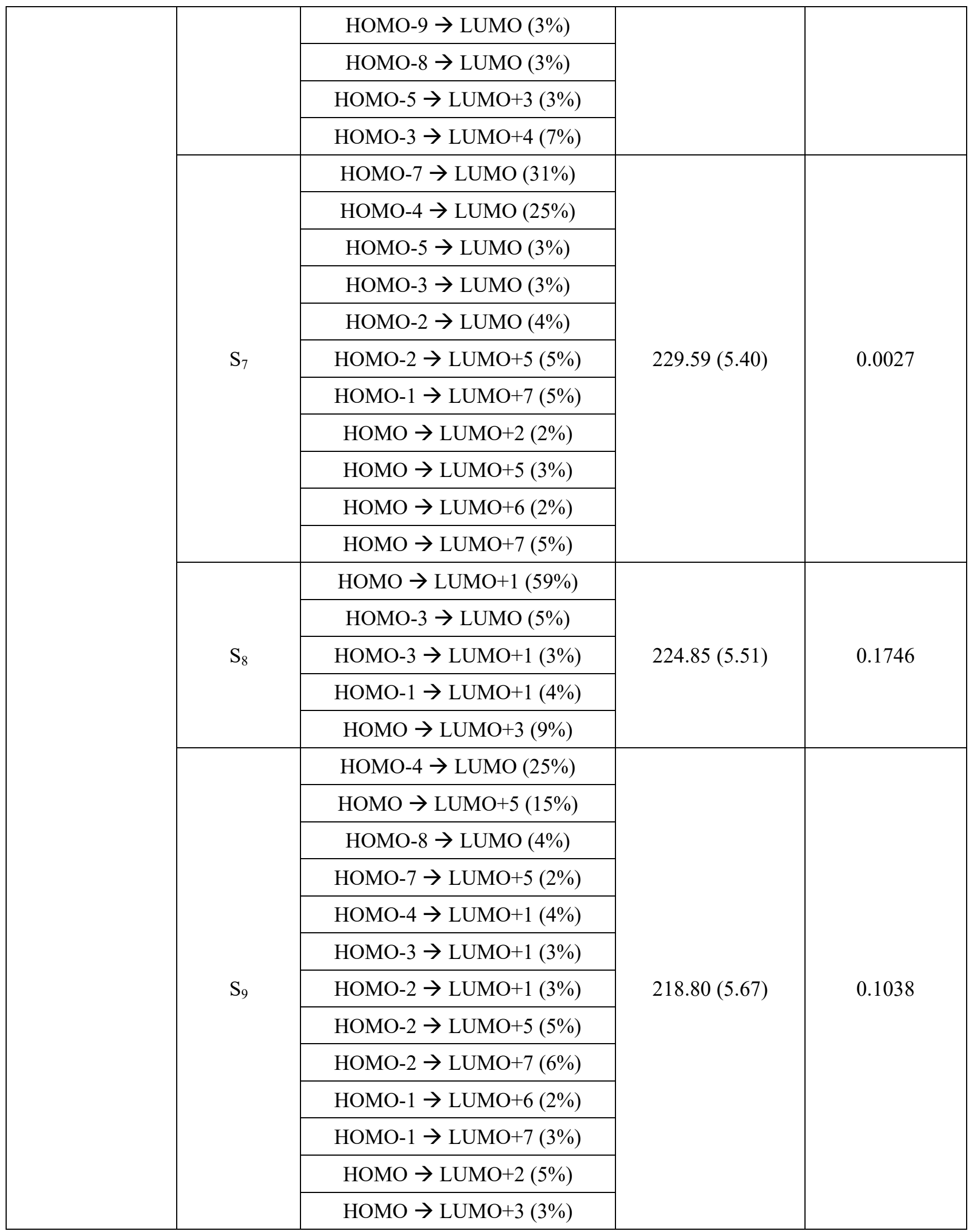




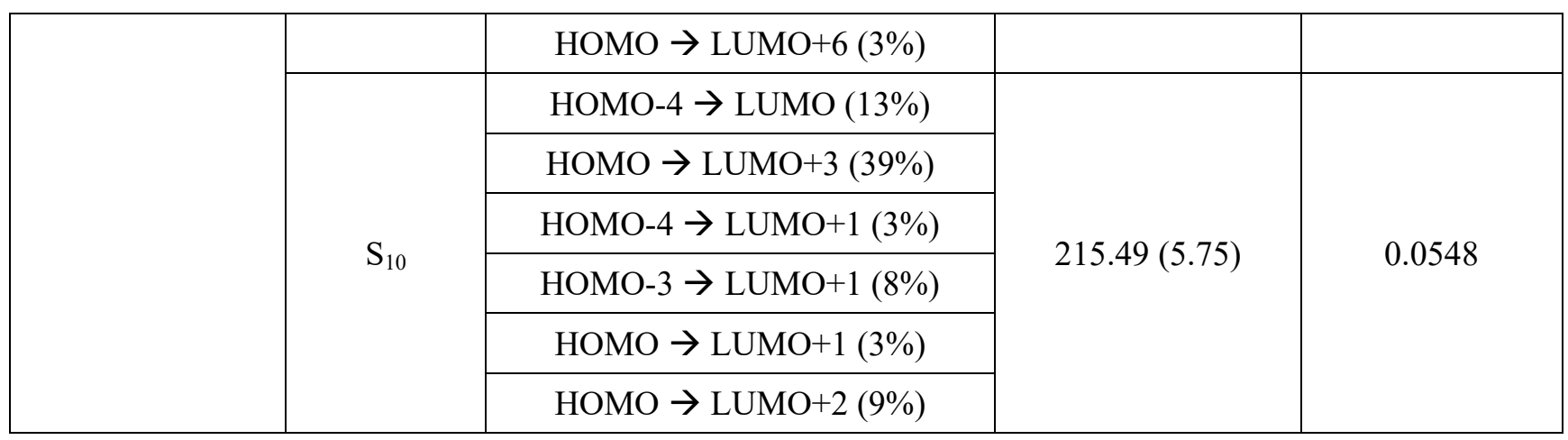

Table S6.1H. TD-DFT calculated electronic transitions for BN-3a along with their corresponding excitation energies and oscillator strengths.

\begin{tabular}{|c|c|c|c|c|}
\hline Compound & Spin State & Transition Configuration & $\begin{array}{c}\text { Excitation Energy } \\
(\mathrm{nm}, \mathrm{eV})\end{array}$ & $\begin{array}{l}\text { Oscillator } \\
\text { Strength }\end{array}$ \\
\hline \multirow{23}{*}{ BN-3a } & $\mathrm{S}_{1}$ & HOMO $\rightarrow$ LUMO (97\%) & $493.21(2.51)$ & 0.0613 \\
\hline & \multirow{3}{*}{$\mathrm{S}_{2}$} & $\mathrm{HOMO} \rightarrow \mathrm{LUMO}+1(89 \%)$ & \multirow{3}{*}{$414.80(2.99)$} & \multirow{3}{*}{0.2375} \\
\hline & & $\mathrm{HOMO} \rightarrow \mathrm{LUMO}+2(4 \%)$ & & \\
\hline & & $\mathrm{HOMO} \rightarrow \mathrm{LUMO}+3(3 \%)$ & & \\
\hline & \multirow{5}{*}{$\mathrm{S}_{3}$} & $\mathrm{HOMO} \rightarrow \mathrm{LUMO}+2(57 \%)$ & \multirow{5}{*}{$341.60(3.63)$} & \multirow{5}{*}{0.0135} \\
\hline & & $\mathrm{HOMO} \rightarrow \mathrm{LUMO}+3(26 \%)$ & & \\
\hline & & $\mathrm{HOMO} \rightarrow \mathrm{LUMO}+1$ (7\%) & & \\
\hline & & $\mathrm{HOMO} \rightarrow \mathrm{LUMO}+4(3 \%)$ & & \\
\hline & & $\mathrm{HOMO} \rightarrow \mathrm{LUMO}+7(2 \%)$ & & \\
\hline & \multirow{2}{*}{$\mathrm{S}_{4}$} & $\mathrm{HOMO} \rightarrow \mathrm{LUMO}+2(33 \%)$ & \multirow{2}{*}{$298.72(4.15)$} & \multirow{2}{*}{0.0015} \\
\hline & & $\mathrm{HOMO} \rightarrow \mathrm{LUMO}+3(65 \%)$ & & \\
\hline & \multirow{4}{*}{$\mathrm{S}_{5}$} & HOMO-1 $\rightarrow$ LUMO (82\%) & \multirow{4}{*}{$280.73(4.42)$} & \multirow{4}{*}{0.3489} \\
\hline & & HOMO-4 $\rightarrow$ LUMO (2\%) & & \\
\hline & & HOMO-2 $\rightarrow$ LUMO (3\%) & & \\
\hline & & $\mathrm{HOMO} \rightarrow \mathrm{LUMO}+6(3 \%)$ & & \\
\hline & \multirow{5}{*}{$\mathrm{S}_{6}$} & $\mathrm{HOMO} \rightarrow \mathrm{LUMO}+5(13 \%)$ & \multirow{5}{*}{$275.21(4.51)$} & \multirow{5}{*}{0.0215} \\
\hline & & $\mathrm{HOMO} \rightarrow \mathrm{LUMO}+6(62 \%)$ & & \\
\hline & & $\mathrm{HOMO} \rightarrow \mathrm{LUMO}+7(16 \%)$ & & \\
\hline & & HOMO-1 $\rightarrow$ LUMO (3\%) & & \\
\hline & & $\mathrm{HOMO} \rightarrow \mathrm{LUMO}+4$ (4\%) & & \\
\hline & \multirow{3}{*}{$\mathrm{S}_{7}$} & $\mathrm{HOMO} \rightarrow \mathrm{LUMO}+5(67 \%)$ & \multirow{3}{*}{$269.09(4.61)$} & \multirow{3}{*}{0.0132} \\
\hline & & $\mathrm{HOMO} \rightarrow \mathrm{LUMO}+6(17 \%)$ & & \\
\hline & & $\mathrm{HOMO} \rightarrow \mathrm{LUMO}+8(6 \%)$ & & \\
\hline
\end{tabular}




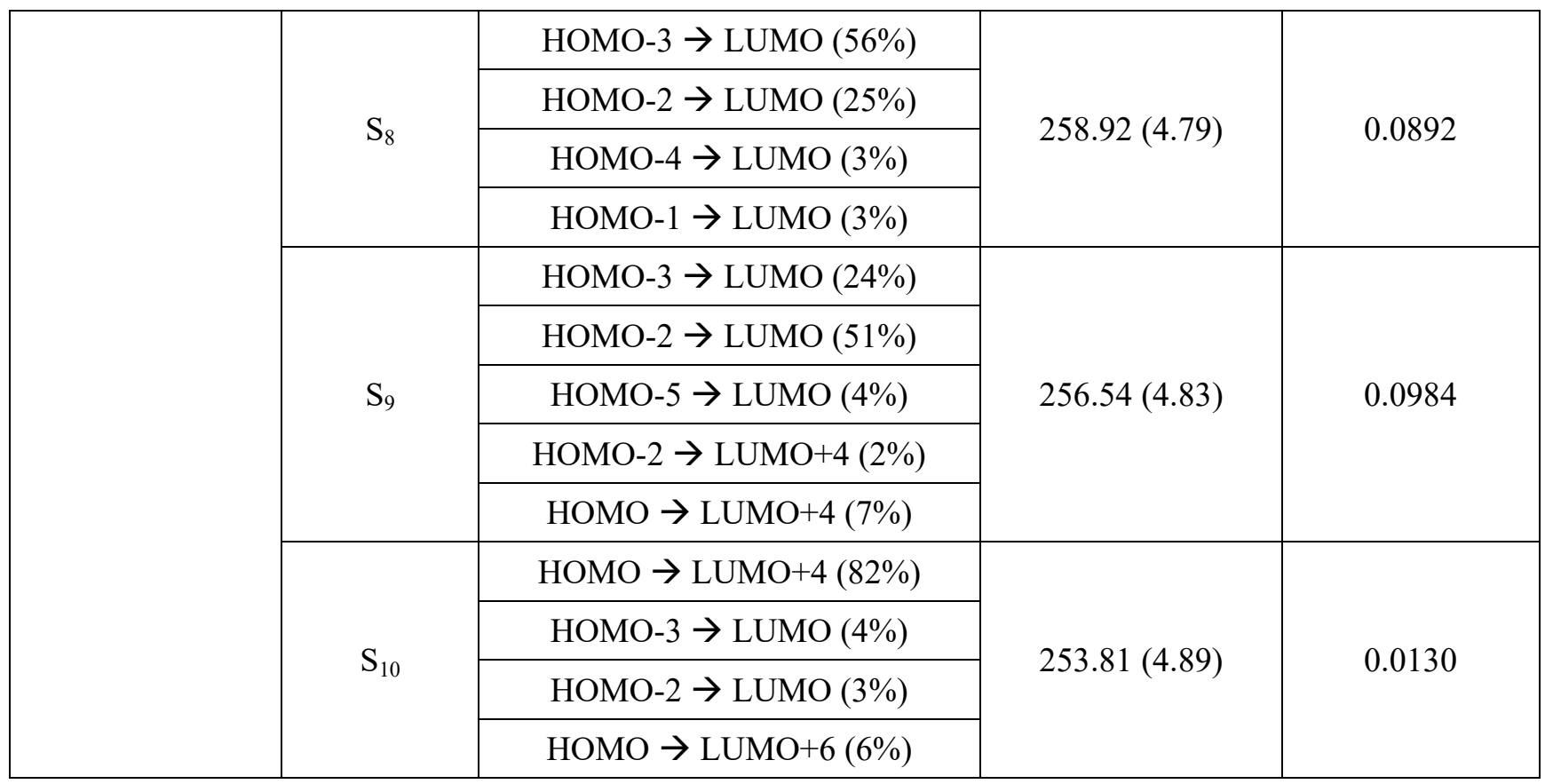

Table S6.1I. TD-DFT calculated electronic transitions for BN-4 along with their corresponding excitation energies and oscillator strengths.

\begin{tabular}{|c|c|c|c|c|}
\hline Compound & Spin State & Transition Configuration & $\begin{array}{c}\text { Excitation Energy } \\
(\mathrm{nm}, \mathrm{eV})\end{array}$ & $\begin{array}{l}\text { Oscillator } \\
\text { Strength }\end{array}$ \\
\hline \multirow{16}{*}{ BN-4 } & \multirow{2}{*}{$\mathrm{S}_{1}$} & HOMO $\rightarrow$ LUMO (88\%) & \multirow{2}{*}{$293.29(4.23)$} & \multirow{2}{*}{0.2101} \\
\hline & & HOMO-4 $\rightarrow$ LUMO (4\%) & & \\
\hline & \multirow{6}{*}{$\mathrm{S}_{2}$} & HOMO-5 $\rightarrow$ LUMO (13\%) & \multirow{6}{*}{$261.21(4.75)$} & \multirow{6}{*}{0.1481} \\
\hline & & HOMO-4 $\rightarrow$ LUMO (47\%) & & \\
\hline & & HOMO $\rightarrow$ LUMO+1 (11\%) & & \\
\hline & & HOMO-3 $\rightarrow$ LUMO (4\%) & & \\
\hline & & HOMO-2 $\rightarrow$ LUMO $(3 \%)$ & & \\
\hline & & HOMO-1 $\rightarrow$ LUMO $(7 \%)$ & & \\
\hline & \multirow{4}{*}{$\mathrm{S}_{3}$} & HOMO-3 $\rightarrow$ LUMO (44\%) & \multirow{4}{*}{$240.08(5.16)$} & \multirow{4}{*}{0.0163} \\
\hline & & HOMO-1 $\rightarrow$ LUMO (41\%) & & \\
\hline & & HOMO-2 $\rightarrow$ LUMO $(5 \%)$ & & \\
\hline & & HOMO-1 $\rightarrow$ LUMO+3 (2\%) & & \\
\hline & \multirow{4}{*}{$\mathrm{S}_{4}$} & HOMO-3 $\rightarrow$ LUMO (27\%) & \multirow{4}{*}{$237.21(5.23)$} & \multirow{4}{*}{0.1117} \\
\hline & & HOMO-1 $\rightarrow$ LUMO (34\%) & & \\
\hline & & $\mathrm{HOMO} \rightarrow \mathrm{LUMO}+1(22 \%)$ & & \\
\hline & & HOMO-2 $\rightarrow$ LUMO $(6 \%)$ & & \\
\hline
\end{tabular}




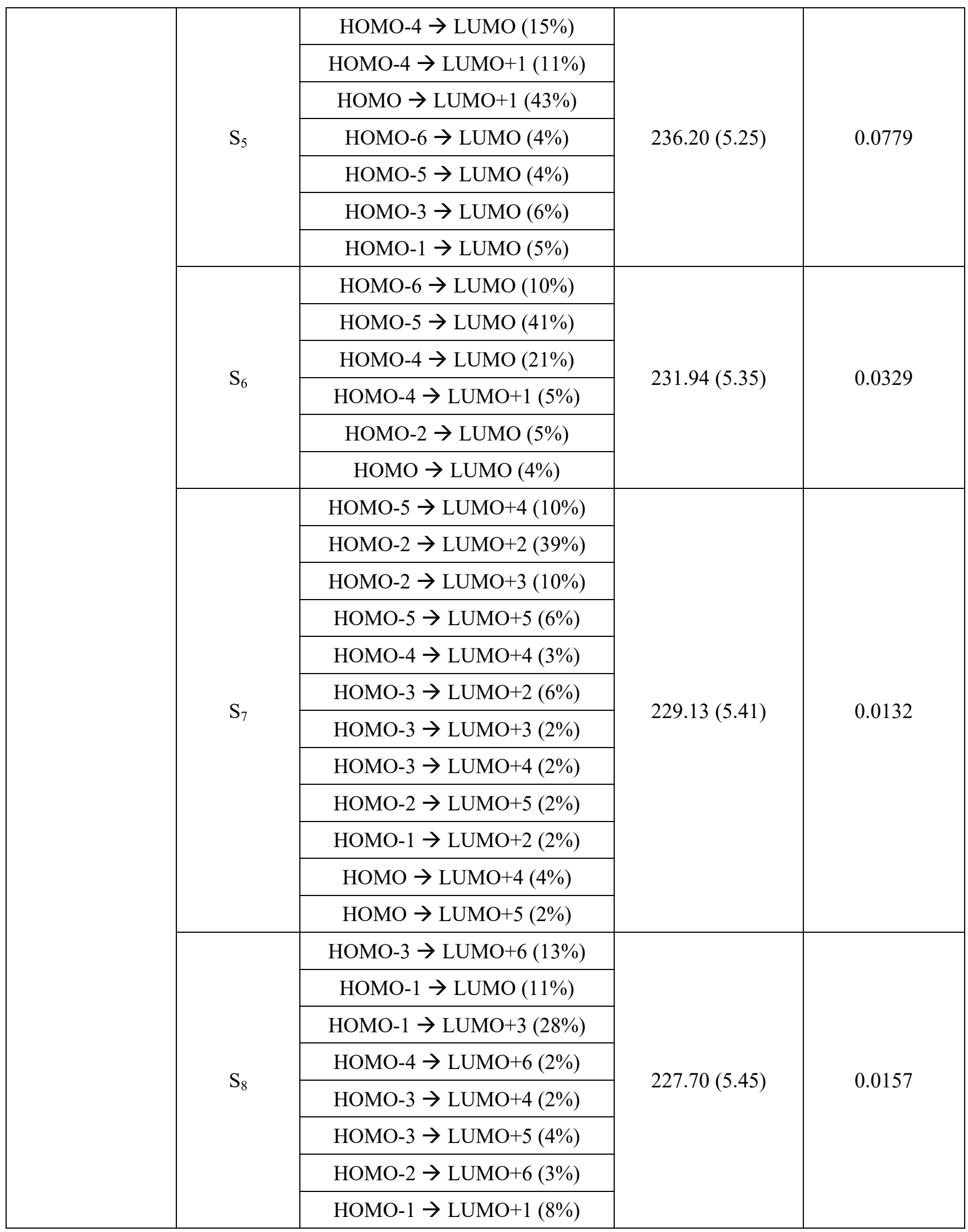




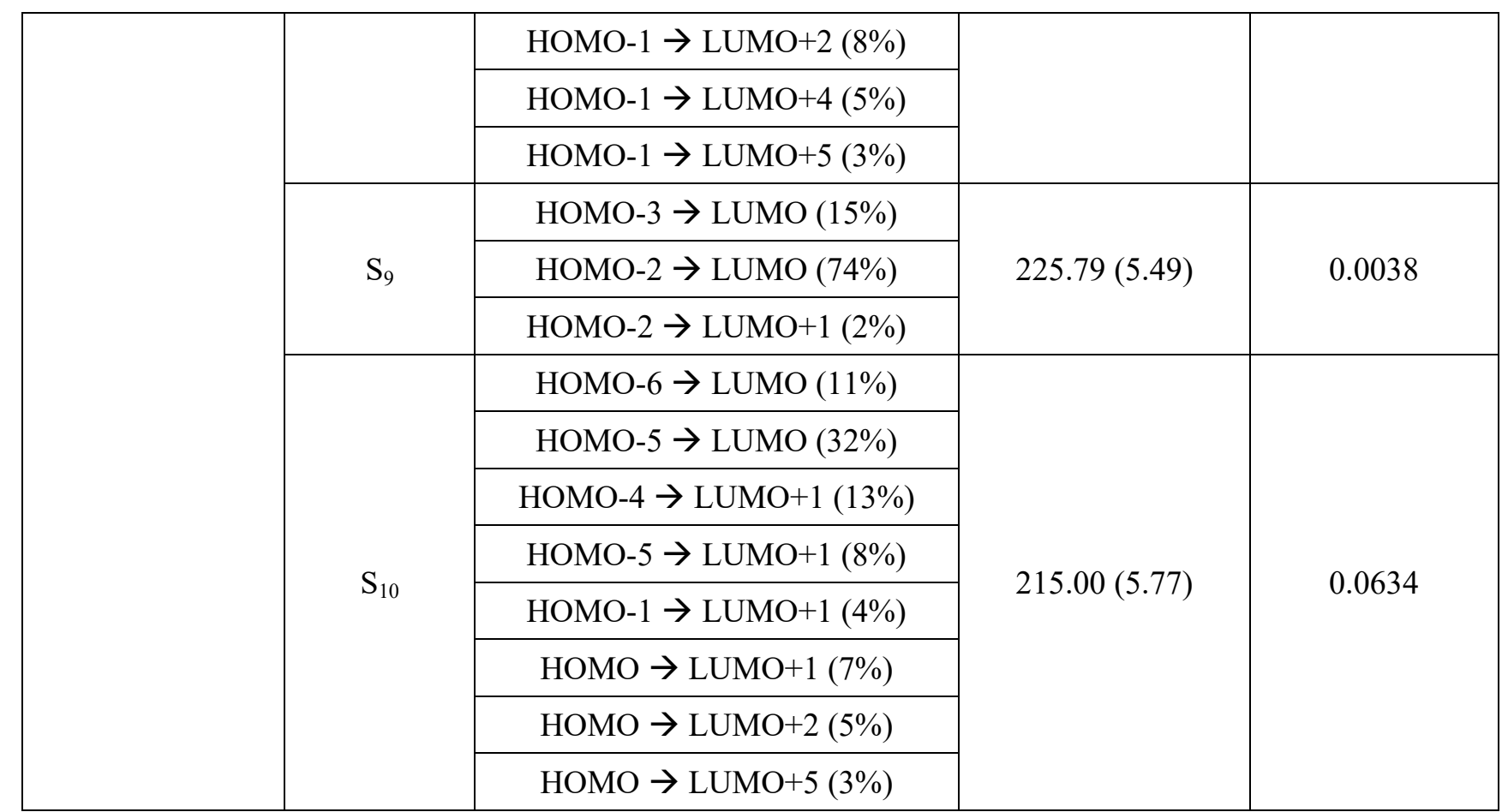

Table S6.1J. TD-DFT calculated electronic transitions for BN-4b along with their corresponding excitation energies and oscillator strengths.

\begin{tabular}{|c|c|c|c|c|}
\hline Compound & Spin State & Transition Configuration & $\begin{array}{c}\text { Excitation Energy } \\
(\mathrm{nm}, \mathrm{eV})\end{array}$ & $\begin{array}{l}\text { Oscillator } \\
\text { Strength }\end{array}$ \\
\hline \multirow{16}{*}{$\mathrm{BN}-4 \mathrm{~b}$} & \multirow{6}{*}{$\mathrm{S}_{1}$} & HOMO-5 $\rightarrow$ LUMO (18\%) & \multirow{6}{*}{$259.71(4.77)$} & \multirow{6}{*}{0.2111} \\
\hline & & HOMO-3 $\rightarrow$ LUMO (34\%) & & \\
\hline & & HOMO-1 $\rightarrow$ LUMO (22\%) & & \\
\hline & & HOMO-6 $\rightarrow$ LUMO $(2 \%)$ & & \\
\hline & & HOMO-4 $\rightarrow$ LUMO (8\%) & & \\
\hline & & HOMO $\rightarrow$ LUMO (5\%) & & \\
\hline & \multirow{5}{*}{$\mathrm{S}_{2}$} & HOMO-4 $\rightarrow$ LUMO (12\%) & \multirow{5}{*}{$245.15(5.06)$} & \multirow{5}{*}{0.0355} \\
\hline & & HOMO-3 $\rightarrow$ LUMO (28\%) & & \\
\hline & & HOMO-1 $\rightarrow$ LUMO (34\%) & & \\
\hline & & HOMO-5 $\rightarrow$ LUMO (5\%) & & \\
\hline & & HOMO-4 $\rightarrow$ LUMO+1 (2\%) & & \\
\hline & \multirow{5}{*}{$\mathrm{S}_{3}$} & HOMO $\rightarrow$ LUMO (73\%) & \multirow{5}{*}{$239.91(5.17)$} & \multirow{5}{*}{0.0137} \\
\hline & & HOMO-4 $\rightarrow$ LUMO (3\%) & & \\
\hline & & HOMO-3 $\rightarrow$ LUMO $(2 \%)$ & & \\
\hline & & HOMO-1 $\rightarrow$ LUMO (7\%) & & \\
\hline & & $\mathrm{HOMO} \rightarrow \mathrm{LUMO}+4(3 \%)$ & & \\
\hline
\end{tabular}




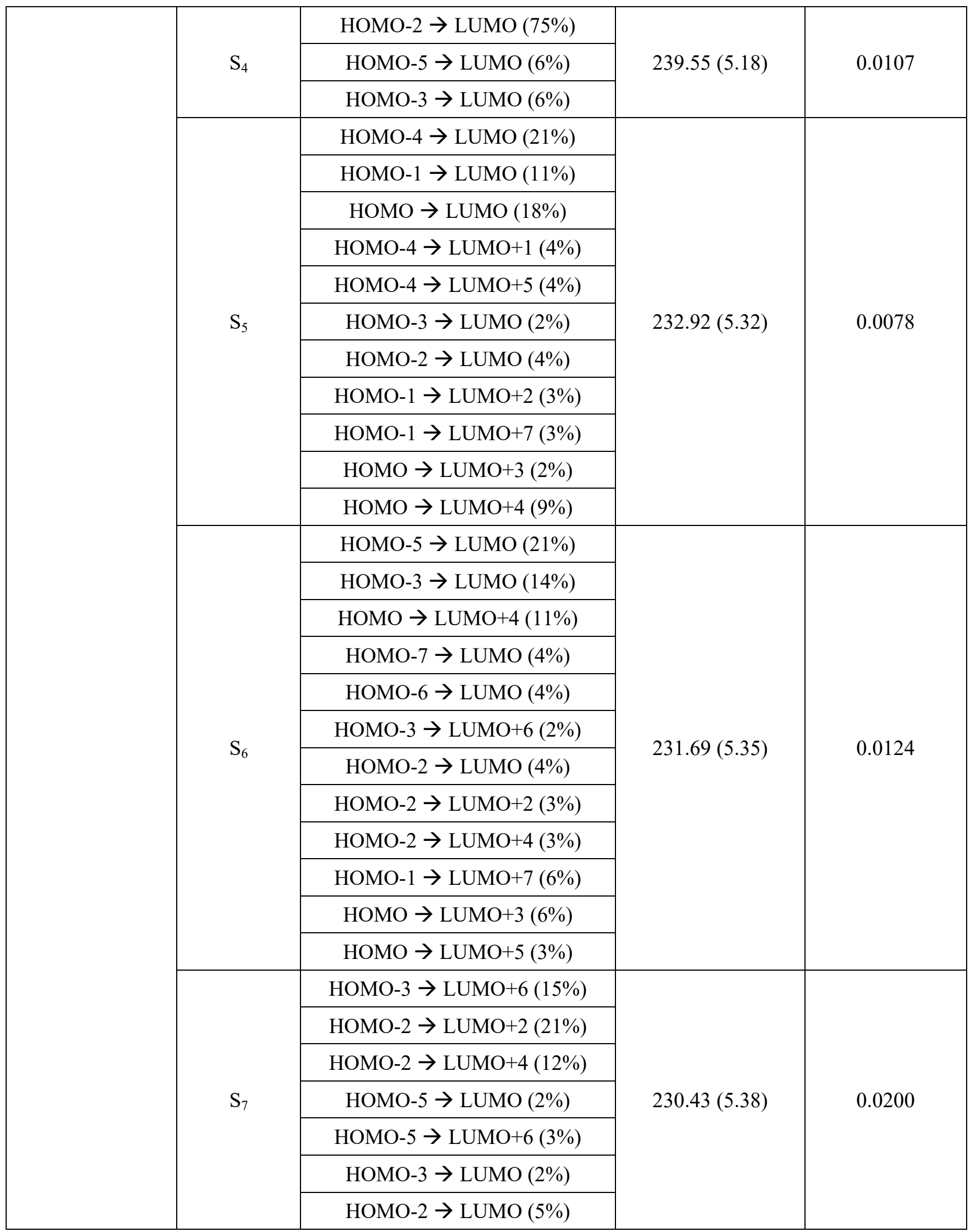




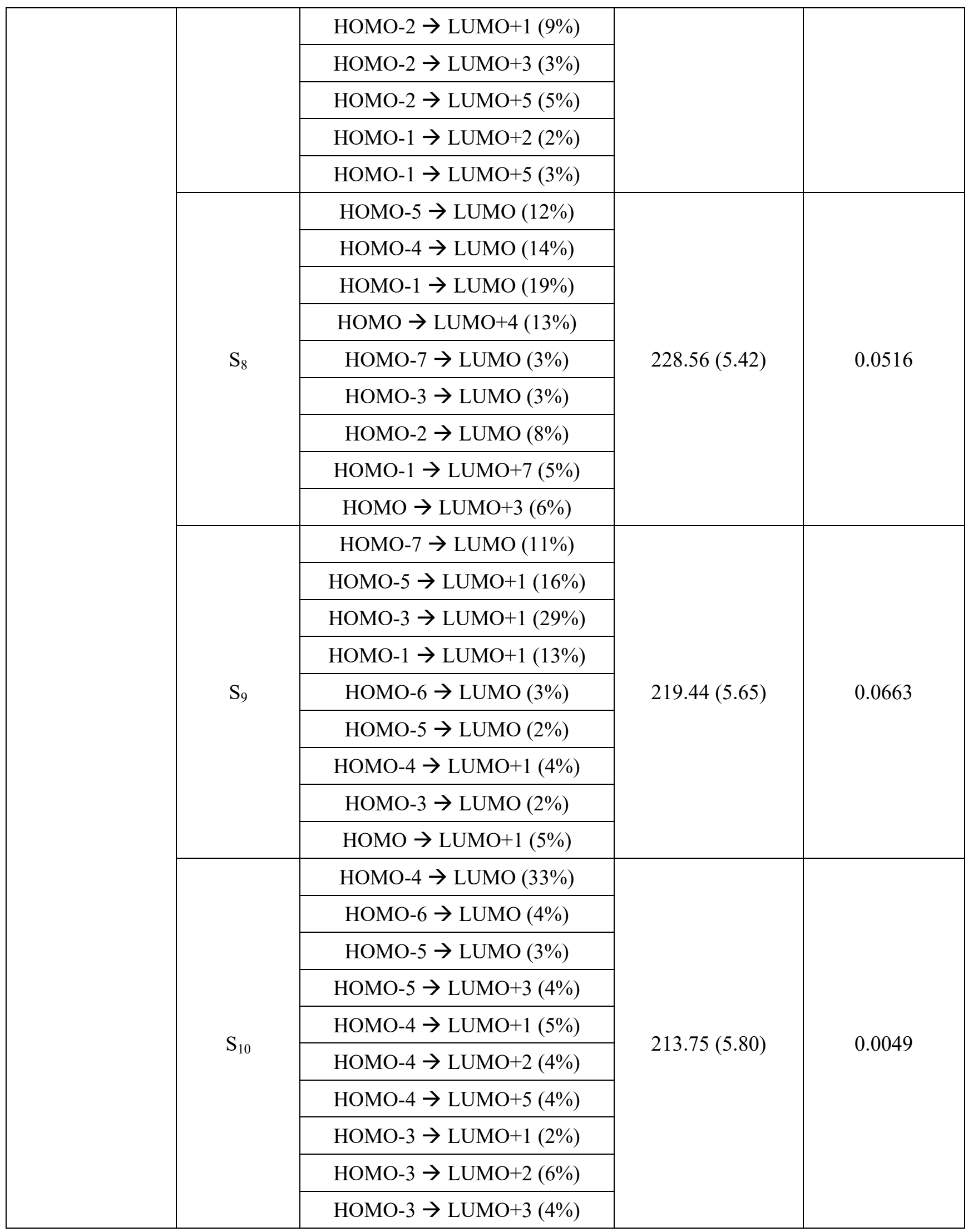




\begin{tabular}{|l|l|l|l|l|}
\hline & & HOMO-1 $\rightarrow$ LUMO+1 $(9 \%)$ & \multirow{2}{*}{} & \\
& & HOMO-1 $\rightarrow$ LUMO $+2(4 \%)$ & & \\
\hline
\end{tabular}

Table S6.1K. TD-DFT calculated electronic transitions for BN-5 along with their corresponding excitation energies and oscillator strengths.

\begin{tabular}{|c|c|c|c|c|}
\hline Compound & Spin State & Transition Configuration & $\begin{array}{c}\text { Excitation Energy } \\
(\mathrm{nm}, \mathrm{eV})\end{array}$ & $\begin{array}{c}\text { Oscillator } \\
\text { Strength }\end{array}$ \\
\hline \multirow{28}{*}{ BN-5 } & \multirow{4}{*}{$\mathrm{S}_{1}$} & HOMO-2 $\rightarrow$ LUMO (20\%) & \multirow{4}{*}{$291.93(4.25)$} & \multirow{4}{*}{0.1953} \\
\hline & & HOMO $\rightarrow$ LUMO $(65 \%)$ & & \\
\hline & & HOMO-4 $\rightarrow$ LUMO $(3 \%)$ & & \\
\hline & & HOMO-1 $\rightarrow$ LUMO $(6 \%)$ & & \\
\hline & \multirow{6}{*}{$\mathrm{S}_{2}$} & HOMO-5 $\rightarrow$ LUMO (42\%) & \multirow{6}{*}{$261.18(4.75)$} & \multirow{6}{*}{0.1789} \\
\hline & & HOMO-1 $\rightarrow$ LUMO (19\%) & & \\
\hline & & HOMO-2 $\rightarrow$ LUMO (7\%) & & \\
\hline & & HOMO-2 $\rightarrow$ LUMO+1 (3\%) & & \\
\hline & & HOMO $\rightarrow$ LUMO (7\%) & & \\
\hline & & $\mathrm{HOMO} \rightarrow \mathrm{LUMO}+1(6 \%)$ & & \\
\hline & \multirow{6}{*}{$\mathrm{S}_{3}$} & HOMO-5 $\rightarrow$ LUMO (17\%) & \multirow{6}{*}{$248.82(4.98)$} & \multirow{6}{*}{0.0402} \\
\hline & & HOMO-2 $\rightarrow$ LUMO (25\%) & & \\
\hline & & HOMO-1 $\rightarrow$ LUMO (13\%) & & \\
\hline & & HOMO $\rightarrow$ LUMO (18\%) & & \\
\hline & & HOMO $\rightarrow$ LUMO+1 (13\%) & & \\
\hline & & HOMO-7 $\rightarrow$ LUMO (3\%) & & \\
\hline & \multirow{10}{*}{$\mathrm{S}_{4}$} & HOMO-4 $\rightarrow$ LUMO (25\%) & \multirow{10}{*}{$238.61(5.20)$} & \multirow{10}{*}{0.0698} \\
\hline & & HOMO-3 $\rightarrow$ LUMO (11\%) & & \\
\hline & & HOMO $\rightarrow$ LUMO+1 (17\%) & & \\
\hline & & HOMO-6 $\rightarrow$ LUMO $(2 \%)$ & & \\
\hline & & HOMO-5 $\rightarrow$ LUMO (4\%) & & \\
\hline & & HOMO-5 $\rightarrow$ LUMO+1 $(5 \%)$ & & \\
\hline & & HOMO-2 $\rightarrow$ LUMO $(7 \%)$ & & \\
\hline & & HOMO-2 $\rightarrow$ LUMO+1 $(8 \%)$ & & \\
\hline & & HOMO-1 $\rightarrow$ LUMO $(6 \%)$ & & \\
\hline & & HOMO-1 $\rightarrow$ LUMO+1 (5\%) & & \\
\hline & \multirow{2}{*}{$\mathrm{S}_{5}$} & HOMO-5 $\rightarrow$ LUMO (15\%) & \multirow{2}{*}{$237.17(5.23)$} & \multirow{2}{*}{0.0714} \\
\hline & & HOMO-4 $\rightarrow$ LUMO (27\%) & & \\
\hline
\end{tabular}




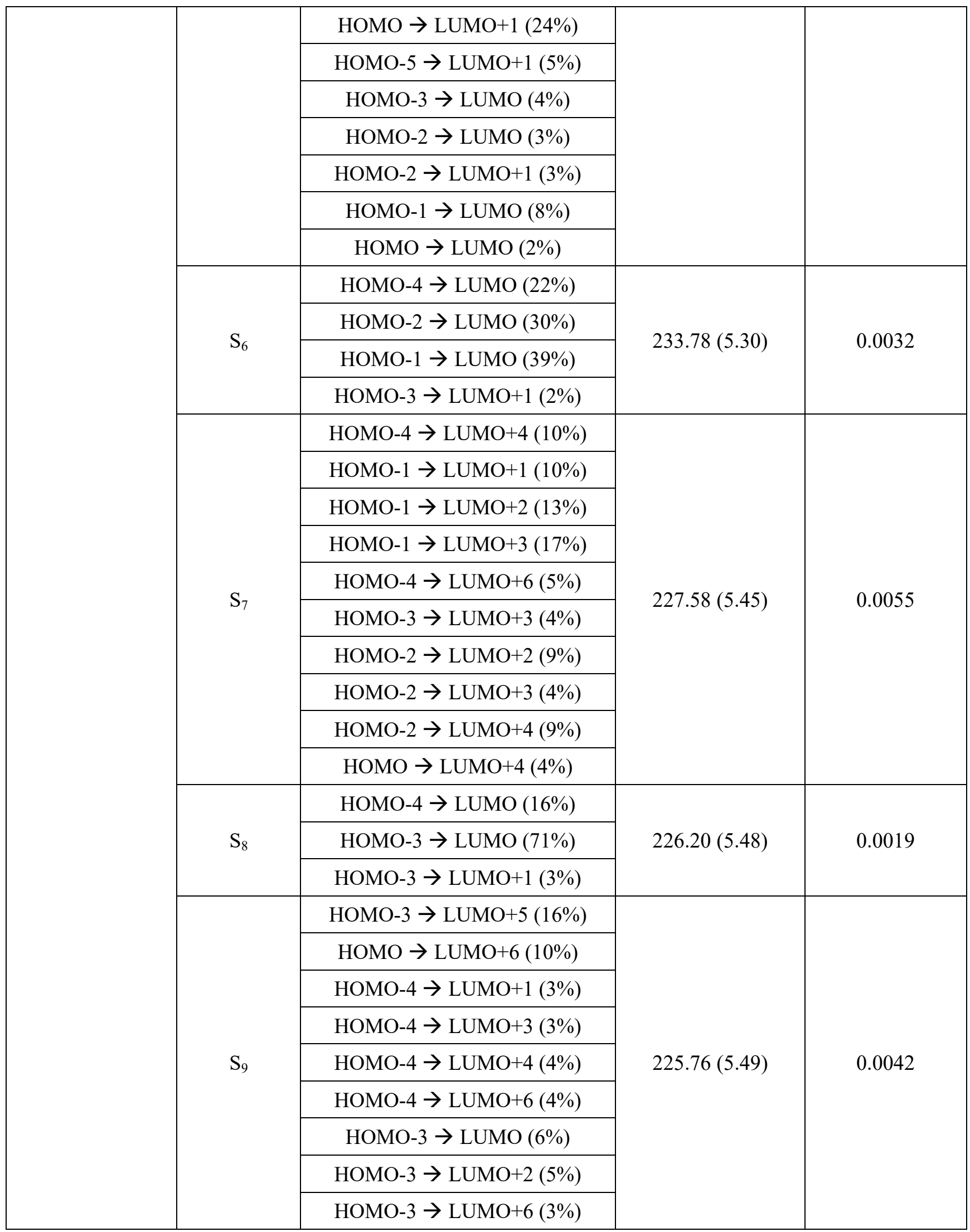




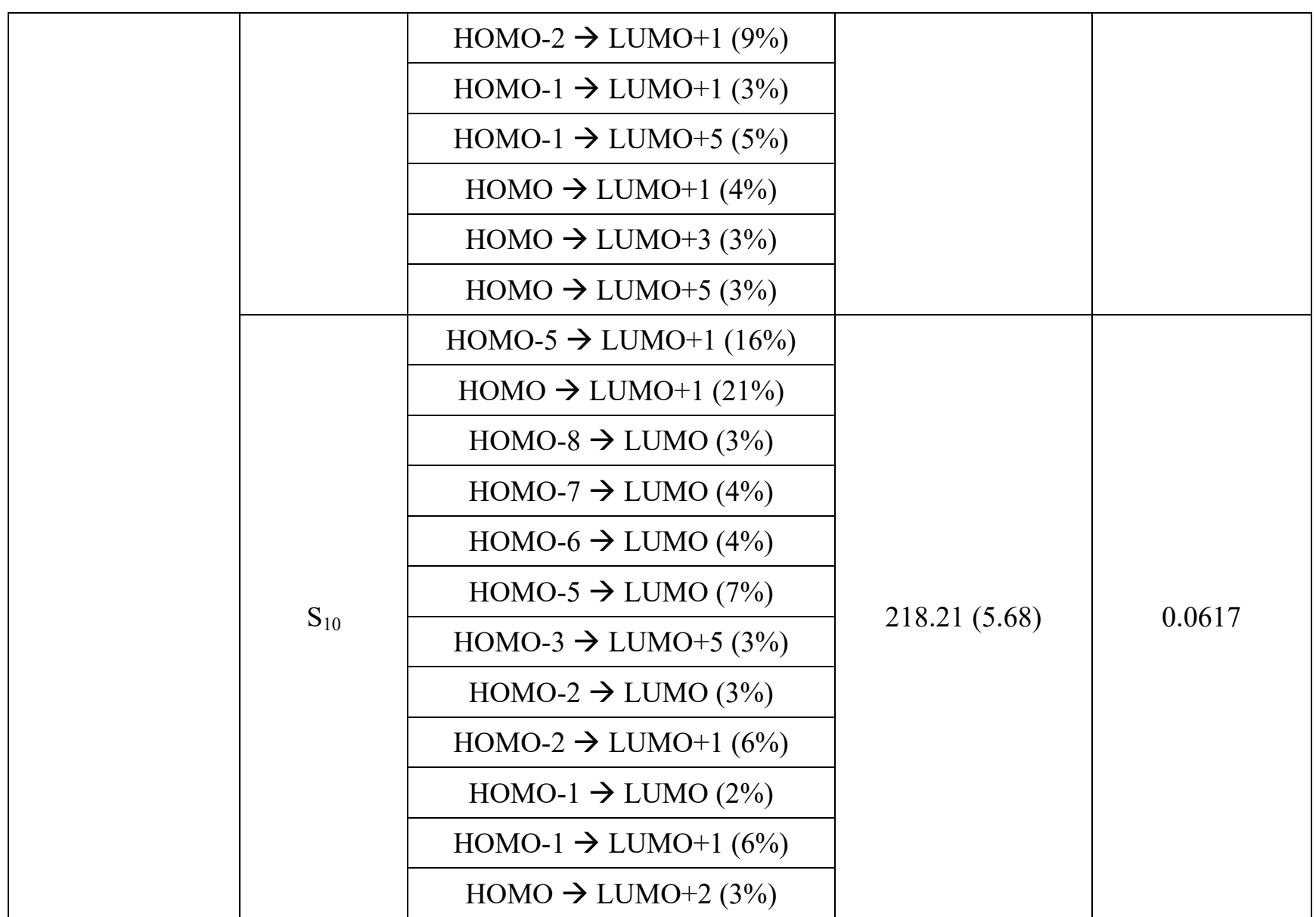

Table S6.1L. TD-DFT calculated electronic transitions for BN-6 along with their corresponding excitation energies and oscillator strengths.

\begin{tabular}{|c|c|c|c|c|}
\hline Compound & Spin State & Transition Configuration & $\begin{array}{l}\text { Excitation Energy } \\
(\mathrm{nm}, \mathrm{eV})\end{array}$ & $\begin{array}{l}\text { Oscillator } \\
\text { Strength }\end{array}$ \\
\hline \multirow{12}{*}{ BN-6 } & \multirow{5}{*}{$\mathrm{S}_{1}$} & HOMO-2 $\rightarrow$ LUMO (10\%) & \multirow{5}{*}{$294.75(4.21)$} & \multirow{5}{*}{0.1569} \\
\hline & & HOMO-1 $\rightarrow$ LUMO (26\%) & & \\
\hline & & HOMO $\rightarrow$ LUMO (47\%) & & \\
\hline & & HOMO-4 $\rightarrow$ LUMO (4\%) & & \\
\hline & & HOMO-3 $\rightarrow$ LUMO (8\%) & & \\
\hline & \multirow{5}{*}{$\mathrm{S}_{2}$} & HOMO-1 $\rightarrow$ LUMO (32\%) & \multirow{5}{*}{$264.25(4.69)$} & \multirow{5}{*}{0.1591} \\
\hline & & $\mathrm{HOMO} \rightarrow \mathrm{LUMO}(42 \%)$ & & \\
\hline & & HOMO-5 $\rightarrow$ LUMO (8\%) & & \\
\hline & & HOMO-4 $\rightarrow$ LUMO (4\%) & & \\
\hline & & HOMO-3 $\rightarrow$ LUMO (9\%) & & \\
\hline & \multirow{2}{*}{$\mathrm{S}_{3}$} & HOMO-5 $\rightarrow$ LUMO (50\%) & \multirow{2}{*}{$257.57(4.81)$} & \multirow{2}{*}{0.0744} \\
\hline & & HOMO-1 $\rightarrow$ LUMO $(13 \%)$ & & \\
\hline
\end{tabular}




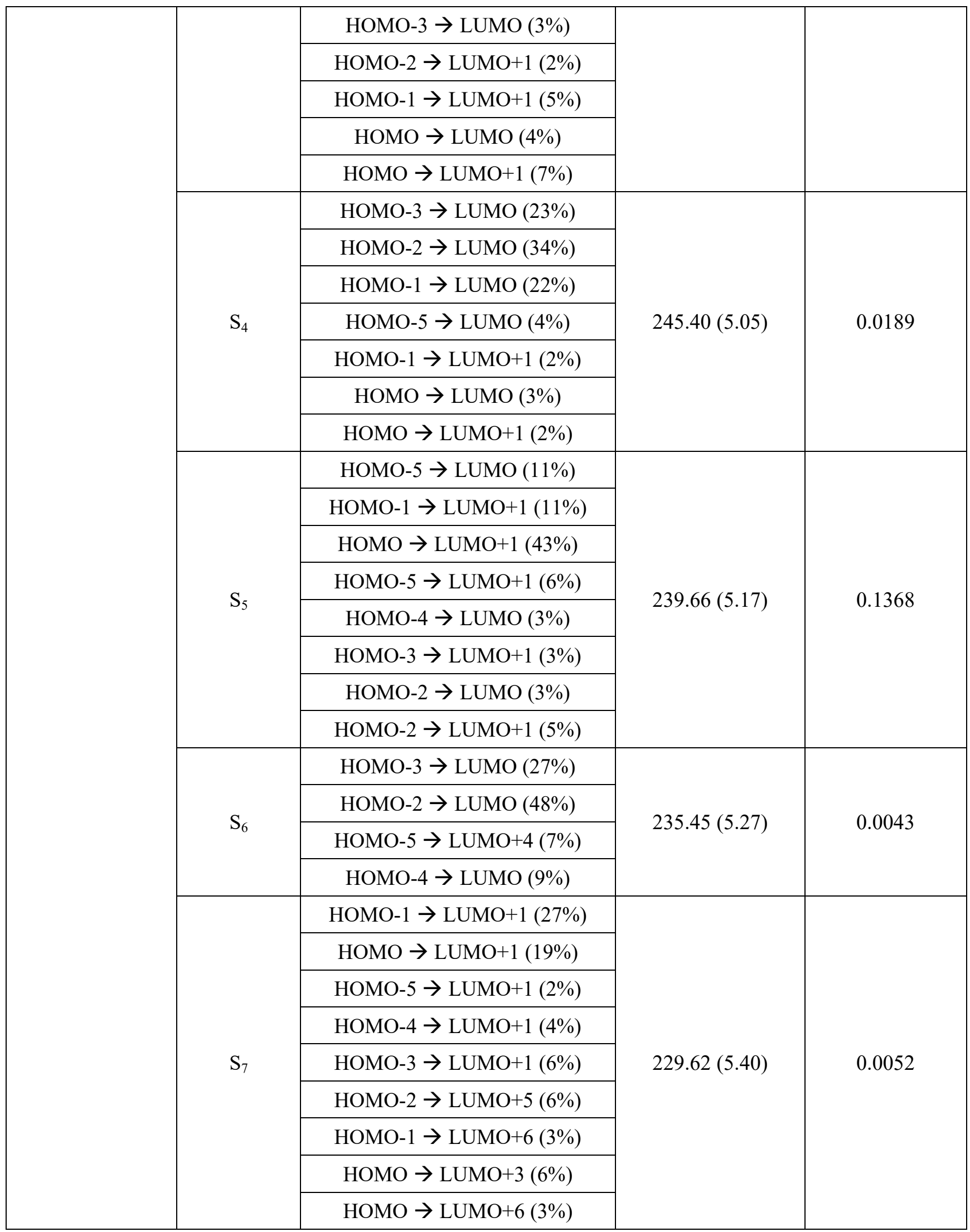




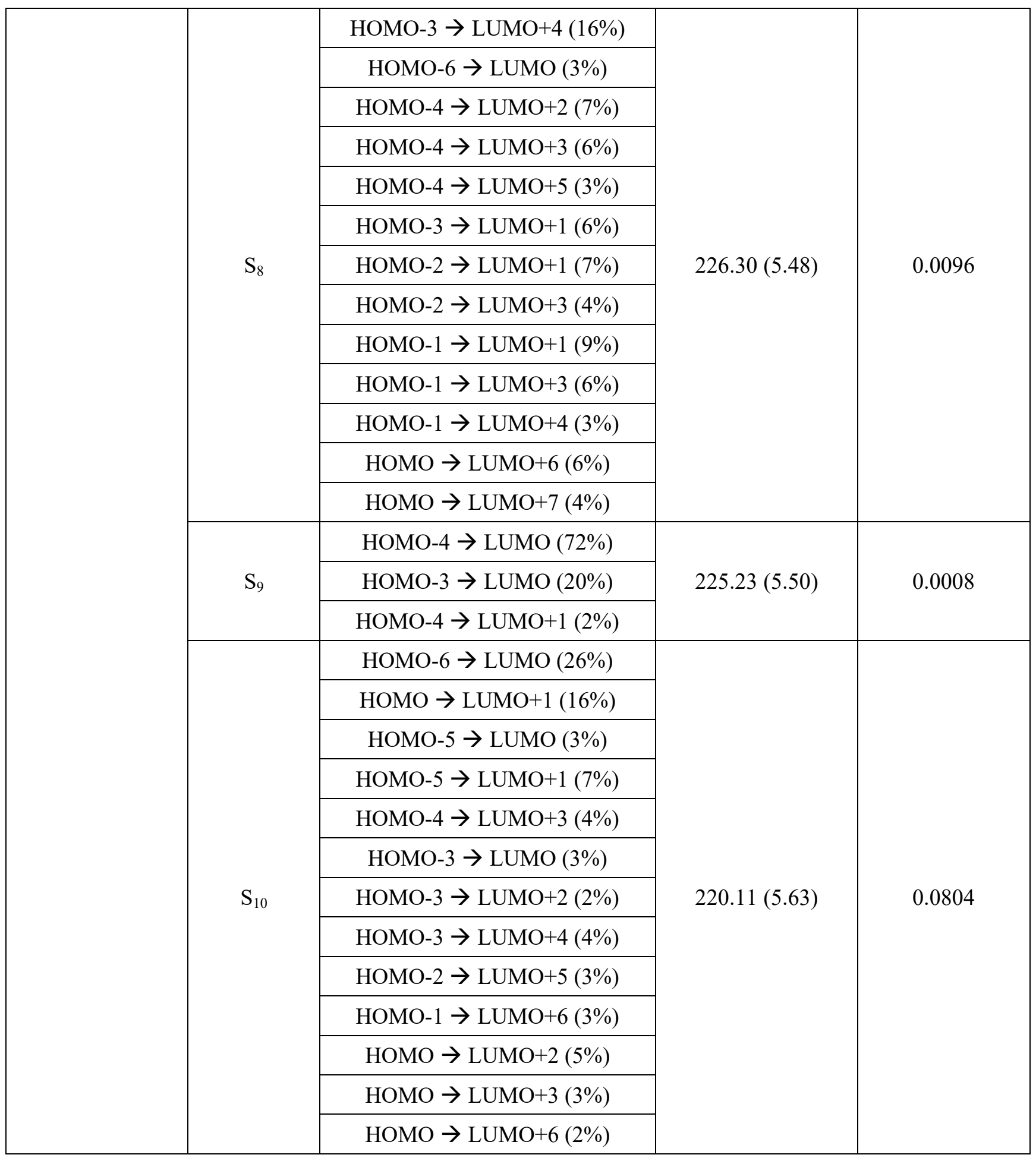


Table S6.1M. Primary molecular orbitals of $\mathbf{B N - 0}$ involved in its electronic transitions.

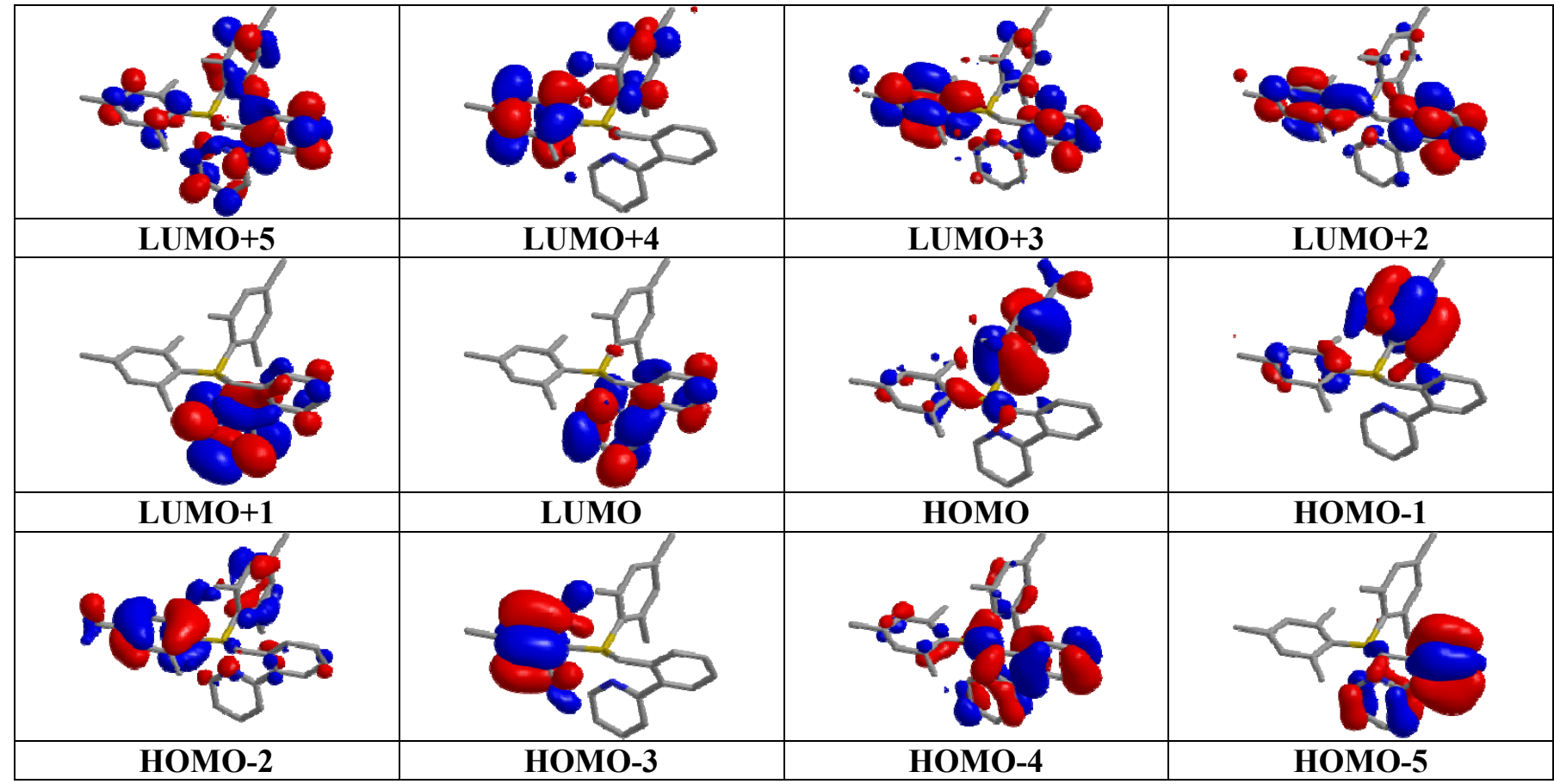

Table S6.1N. Primary molecular orbitals of BN-phen-0 involved in its electronic transitions.

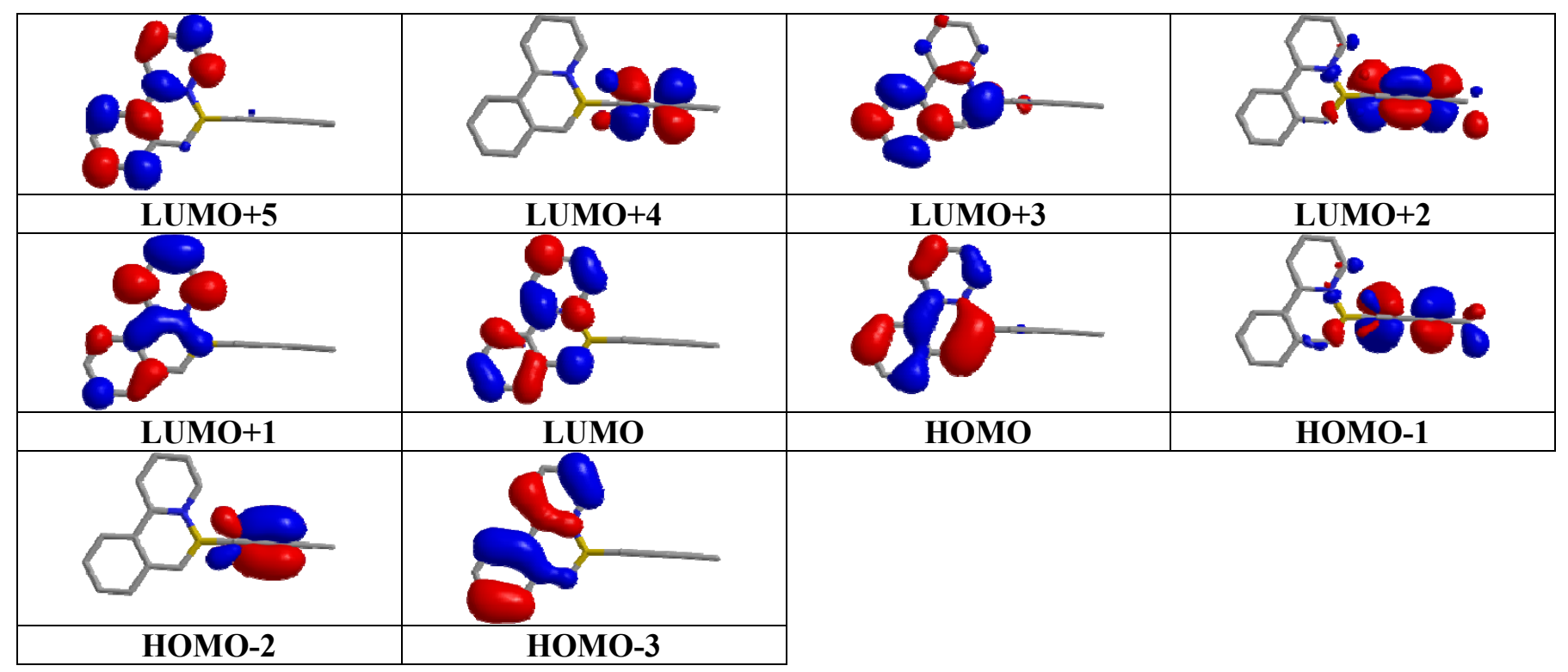


Table S6.10. Primary molecular orbitals of BN-1 involved in its electronic transitions.

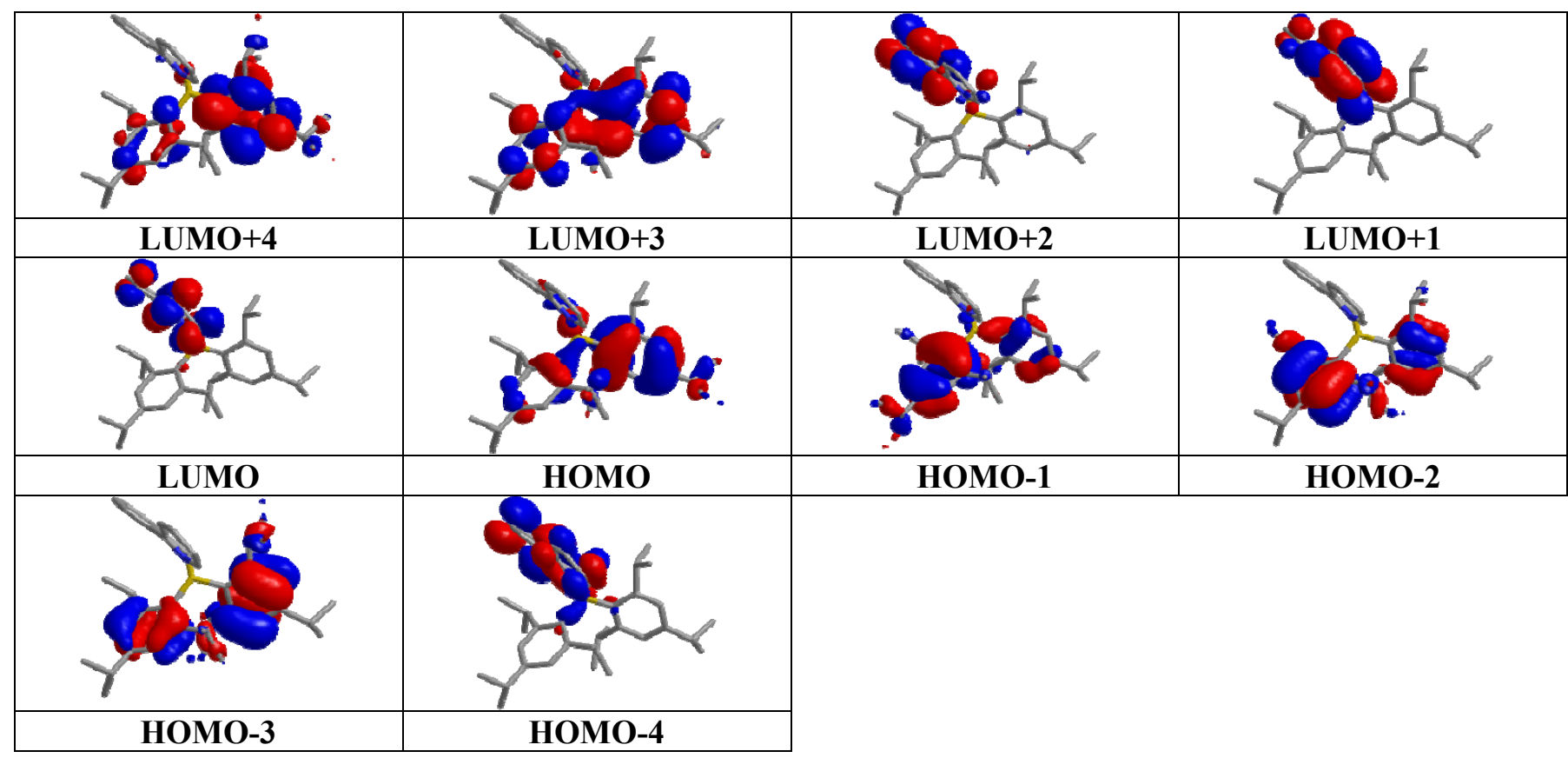

Table S6.1P. Primary molecular orbitals of BN-phen-1 involved in its electronic transitions.

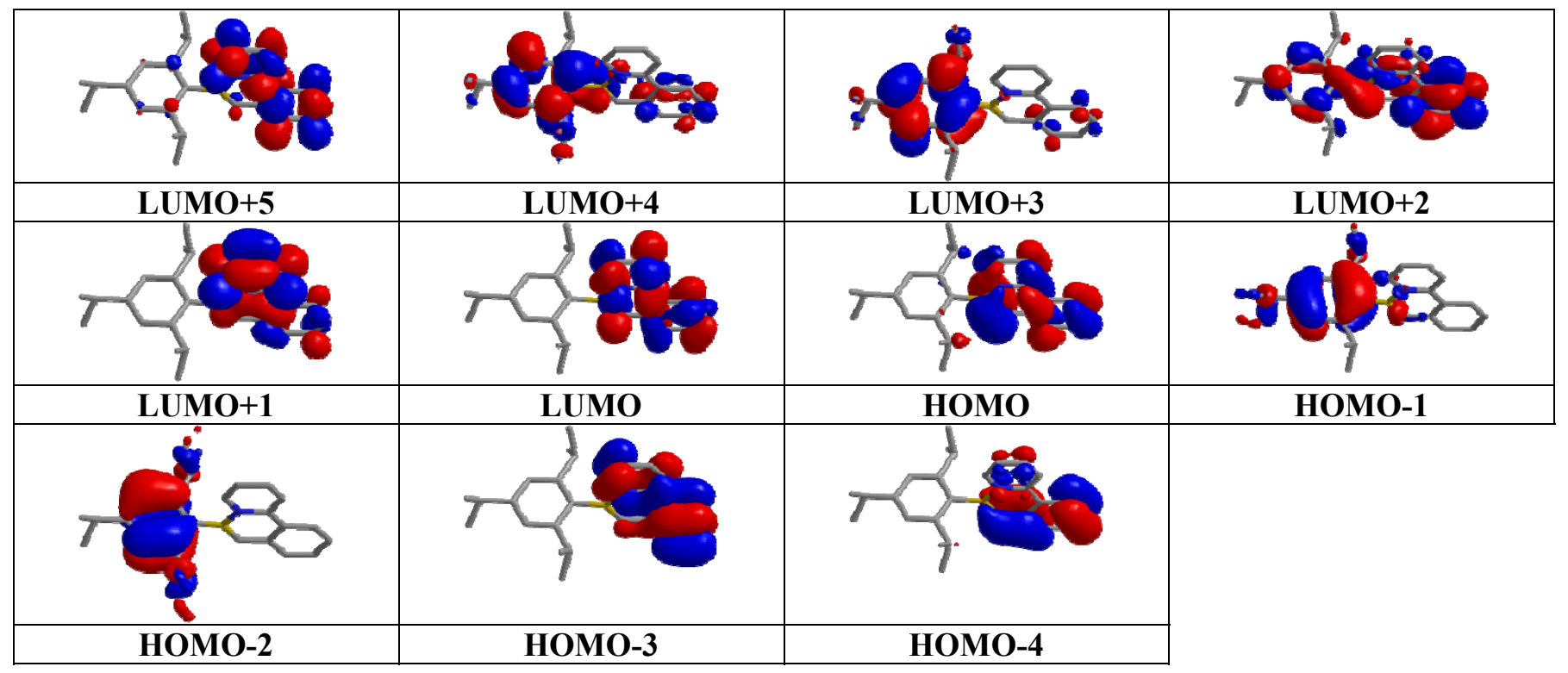


Table S6.1Q. Primary molecular orbitals of BN-2 involved in its electronic transitions.

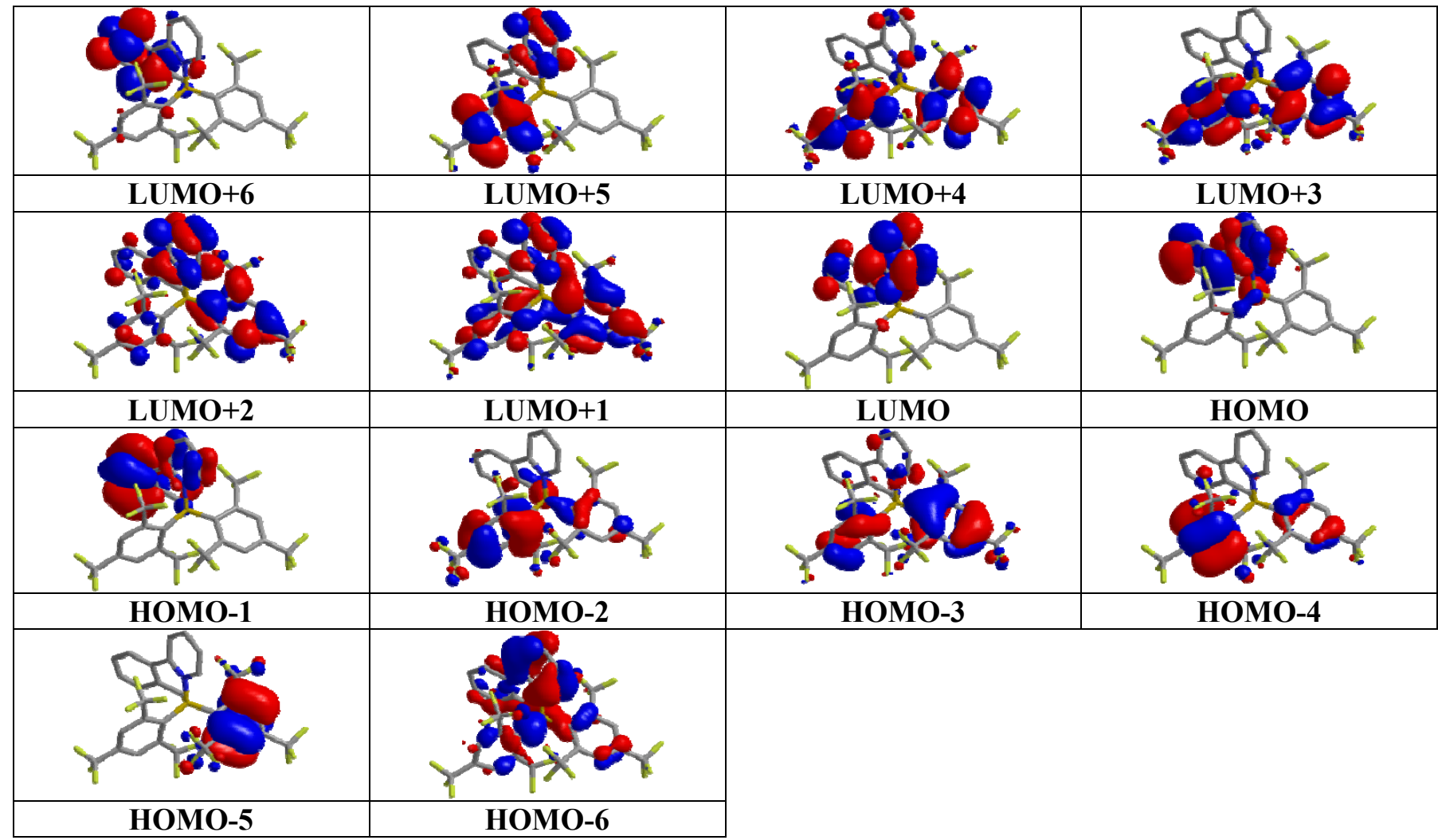

Table S6.1R. Primary molecular orbitals of BN-2a involved in its electronic transitions.

\begin{tabular}{|l|c|c|}
\hline & \\
\hline & & \\
\hline
\end{tabular}


Table S6.1S. Primary molecular orbitals of BN-3 involved in its electronic transitions.

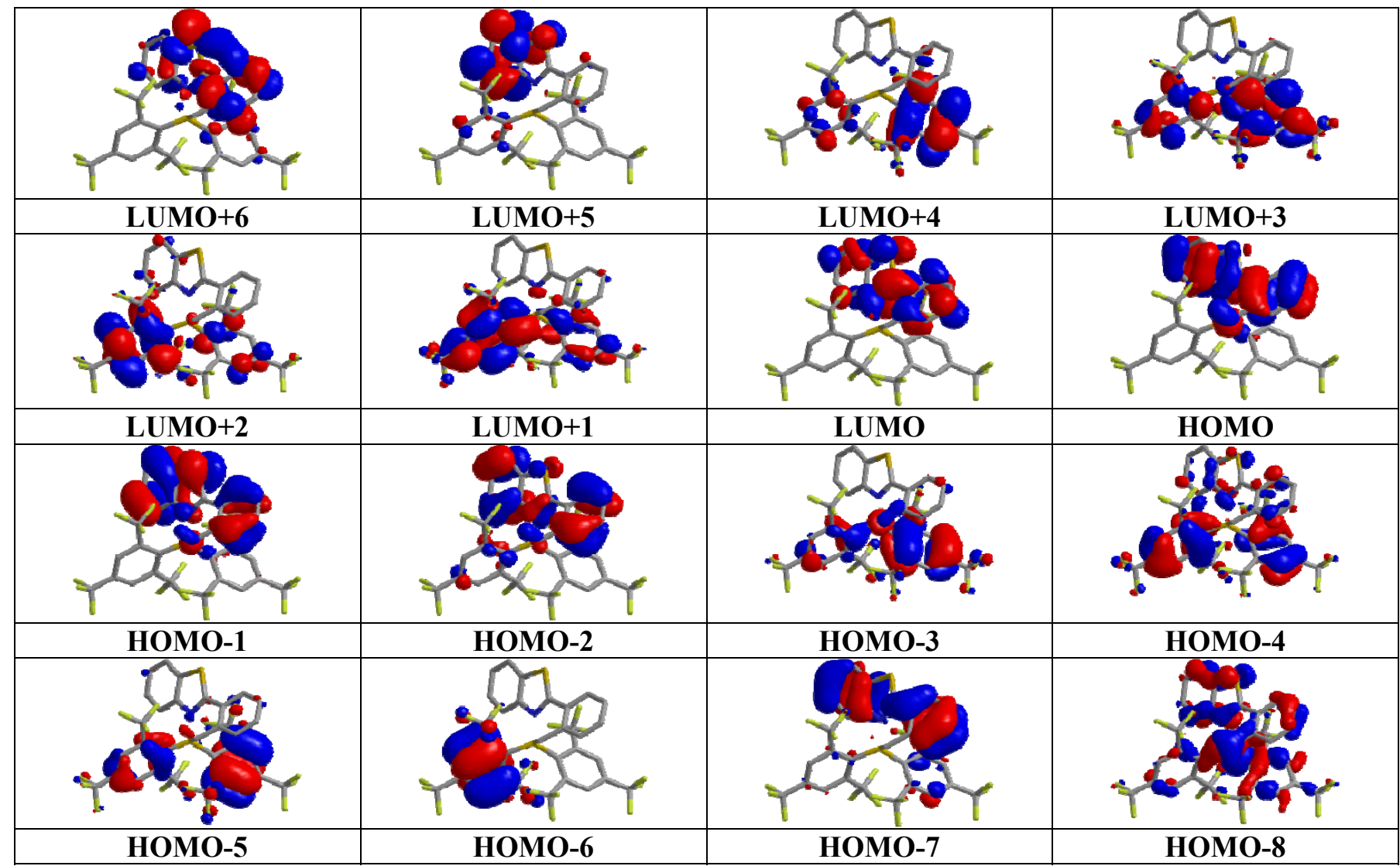

Table S6.1T. Primary molecular orbitals of BN-3a involved in its electronic transitions.

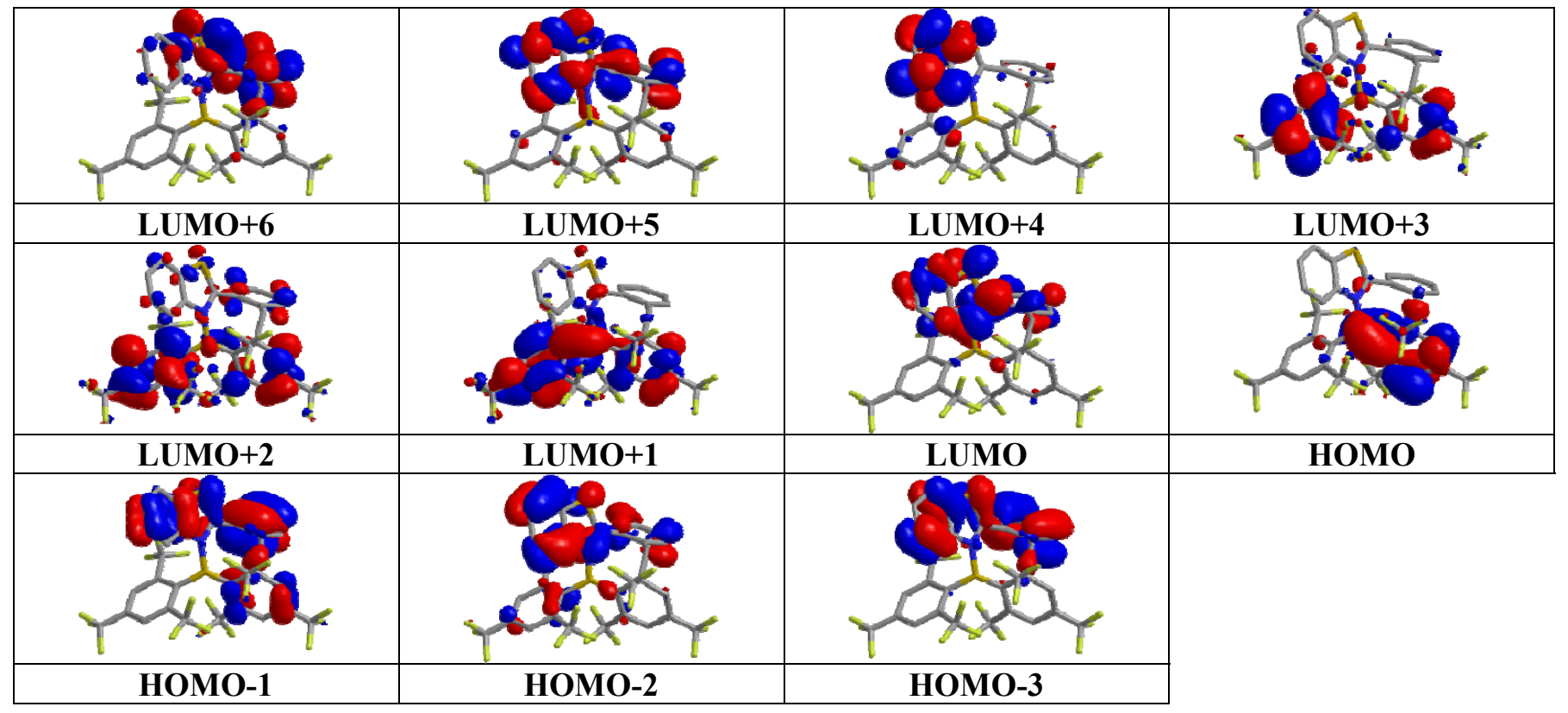


Table S6.1U. Primary molecular orbitals of $\mathbf{B N}-4$ involved in its electronic transitions.

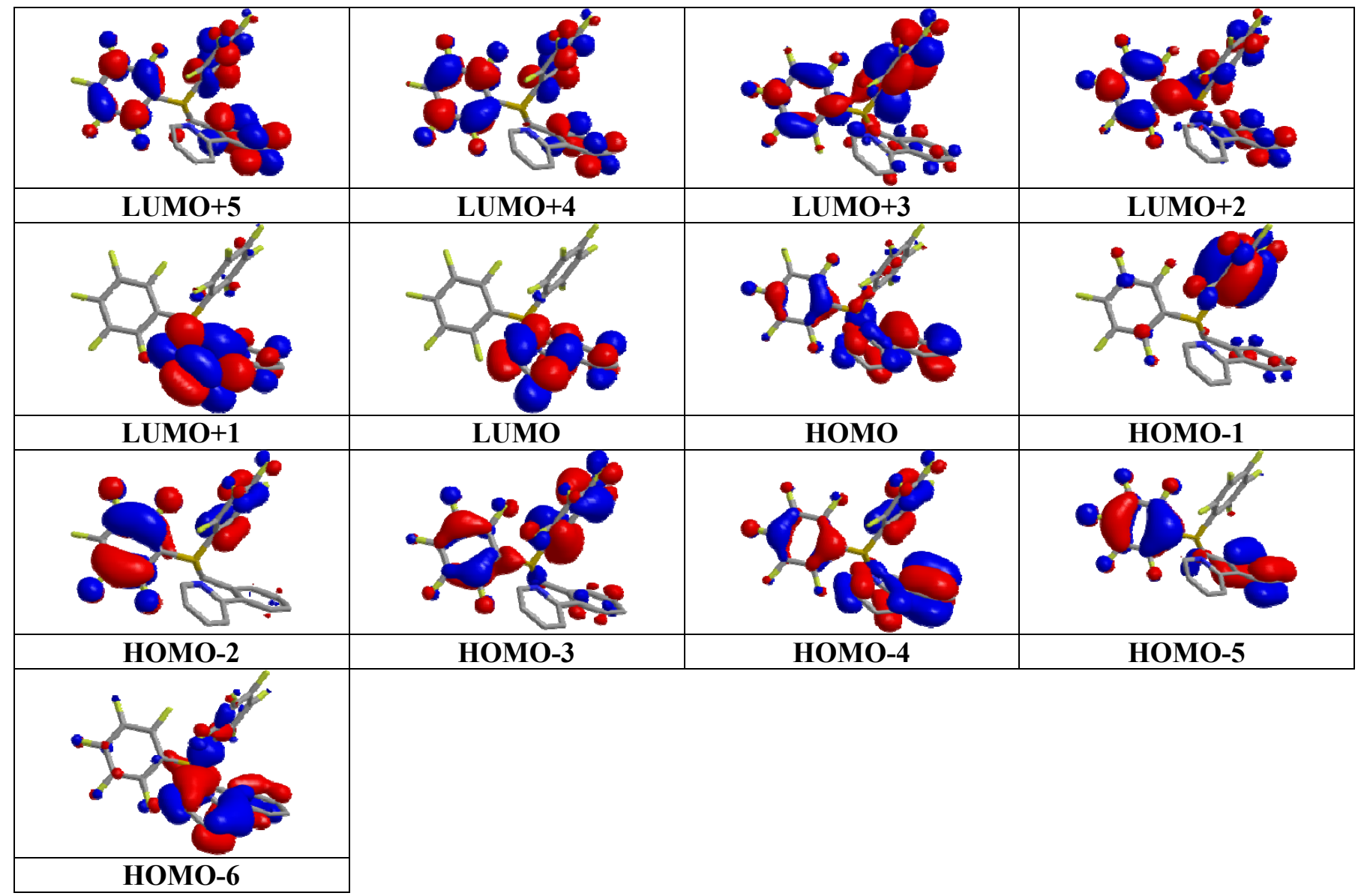

Table S6.1V. Primary molecular orbitals of $\mathbf{B N}-\mathbf{4 b}$ involved in its electronic transitions.

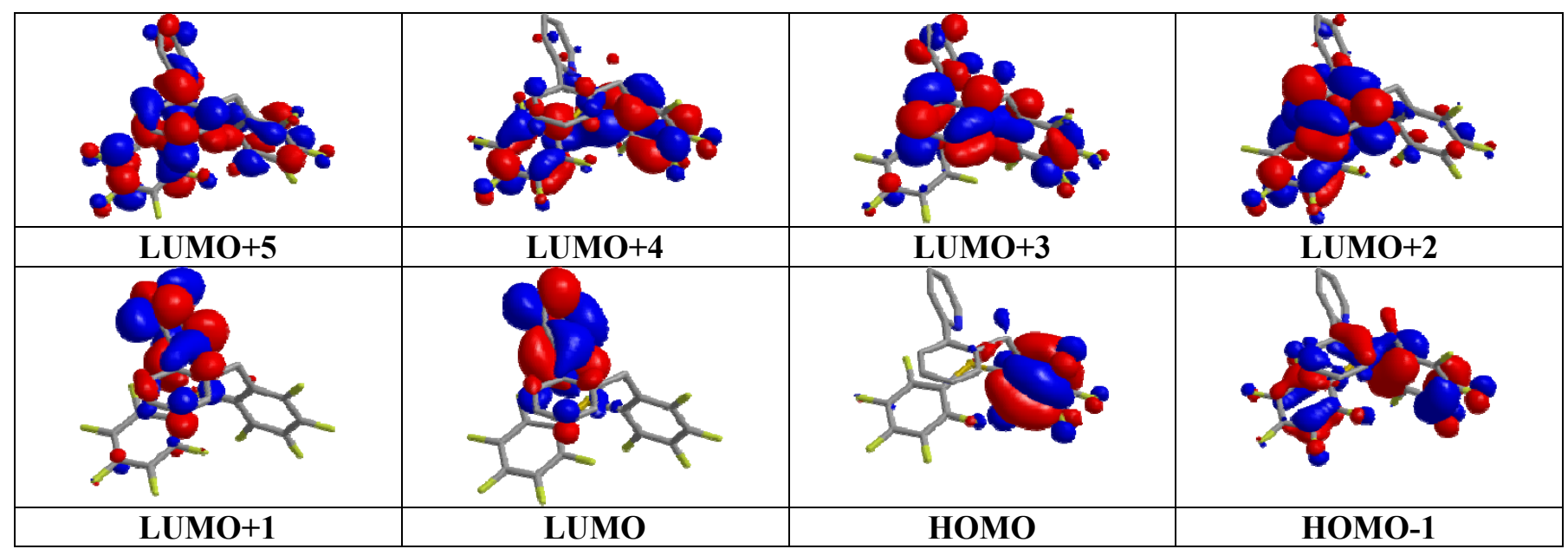




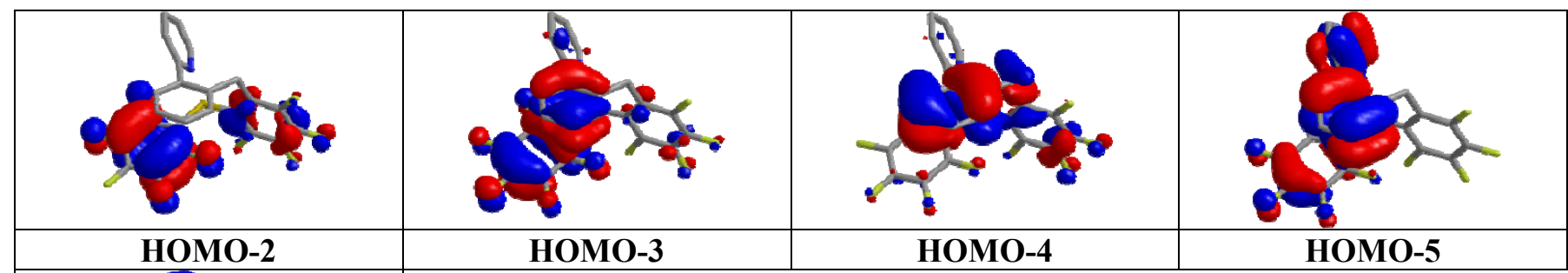

Table S6.1W. Primary molecular orbitals of BN-5 involved in its electronic transitions.

\begin{tabular}{|c|c|c|c|}
\hline LUMO+6 & LUMO+5 & LUMO+4 & LUMO+3 \\
\hline LUMO+2 & LUMO+1 & LUMO & HOMO \\
\hline HOMO-1 & HOMO-2 & HOMO-3 & HOMO-4 \\
\hline HOMO-5 & HOMO-6 & HOMO-7 & HOMO-8 \\
\hline
\end{tabular}


Table S6.1X. Primary molecular orbitals of $\mathbf{B N}-5$ involved in its electronic transitions.

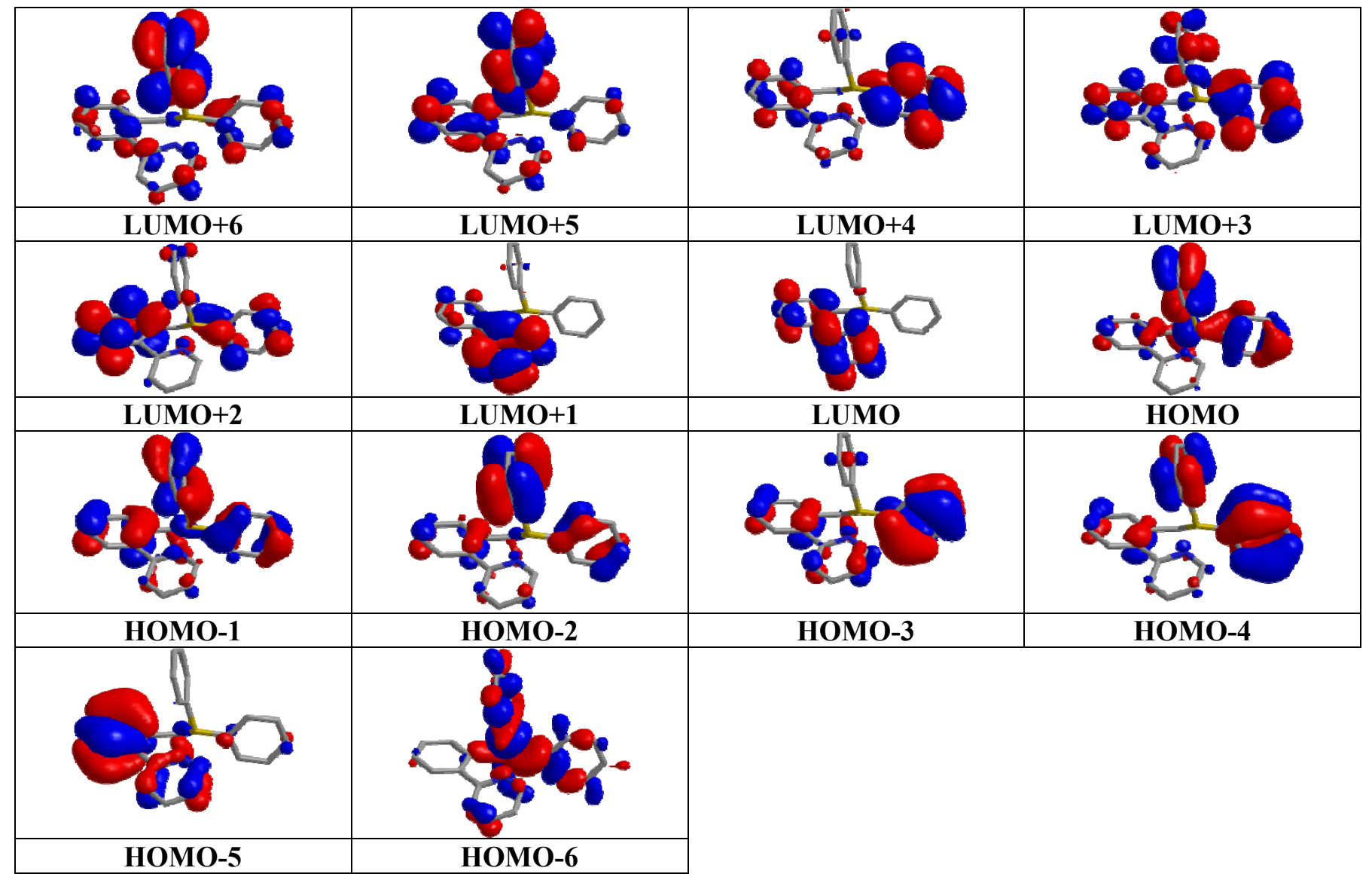



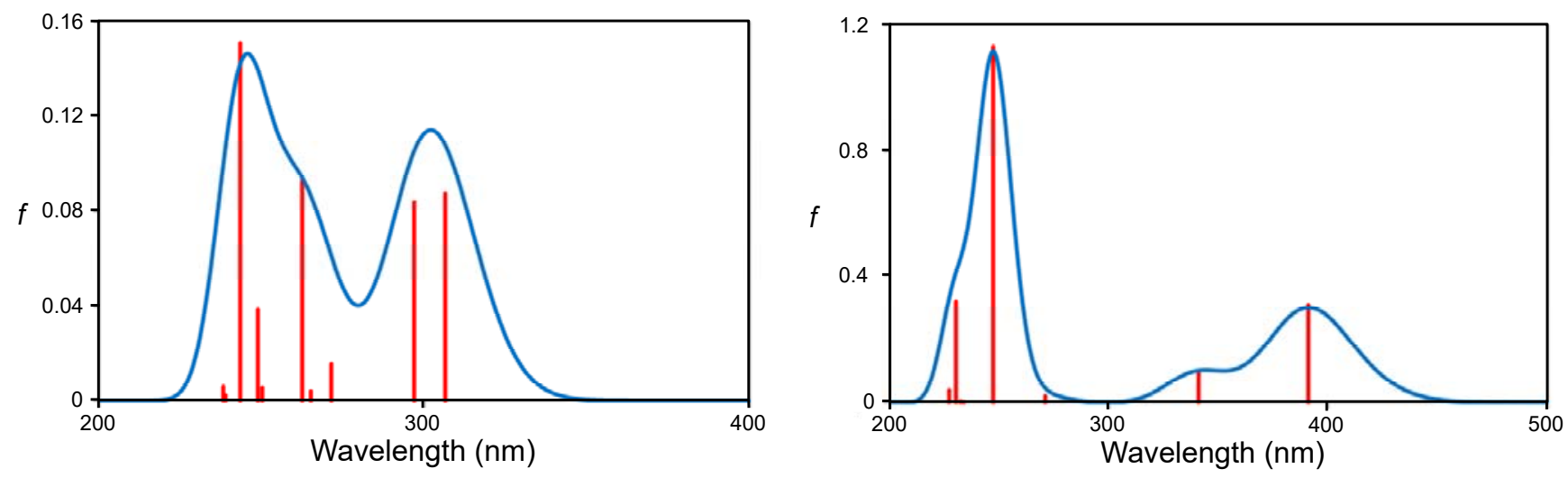

Figure S6.1E. Predicted UV-Vis spectra of BN-0 and BN-phen-0. ${ }^{19}$
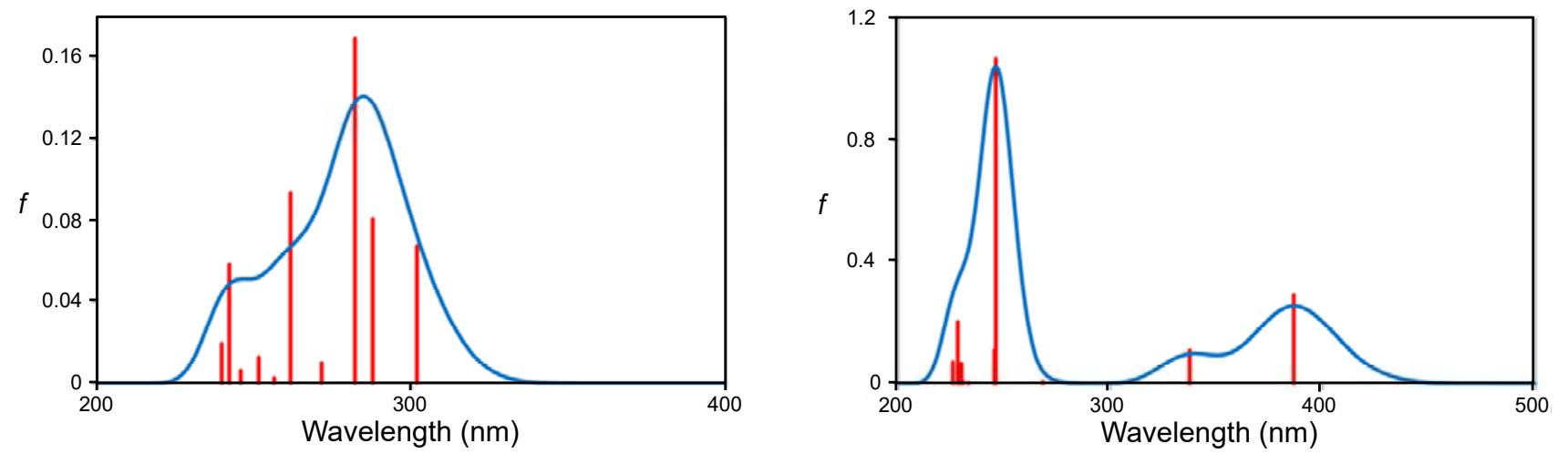

Figure S6.1F. Predicted UV-Vis spectra of BN-1 and BN-phen-1. ${ }^{19}$
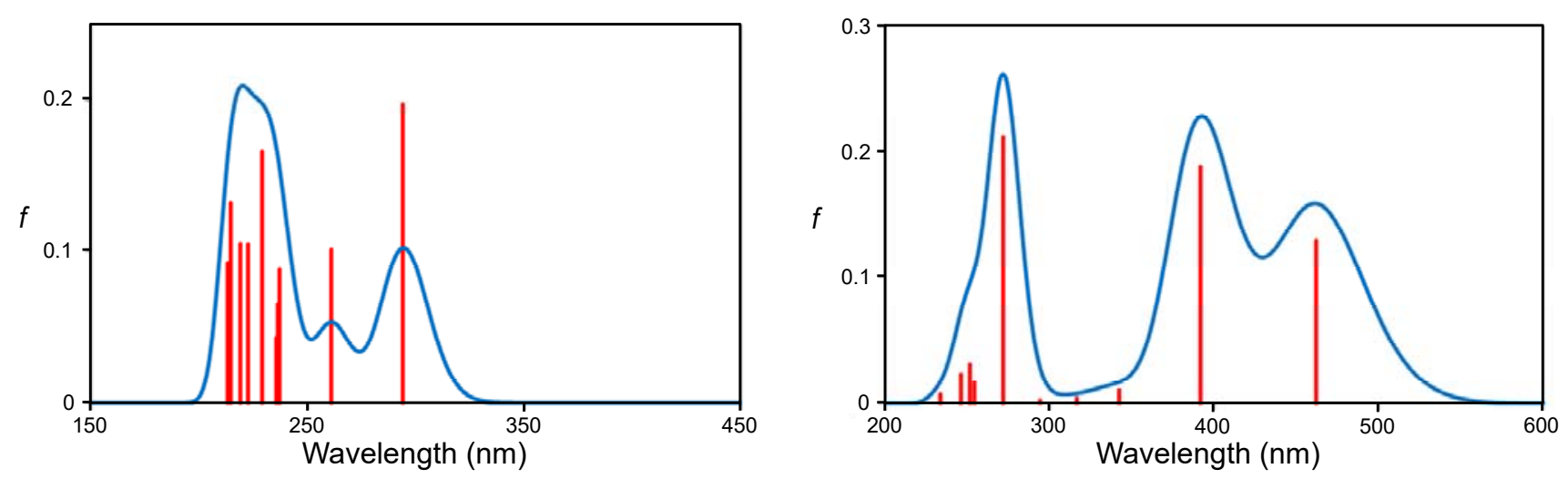

Figure S6.1G. Predicted UV-Vis spectra of BN-2 and BN-2a. ${ }^{19}$ 

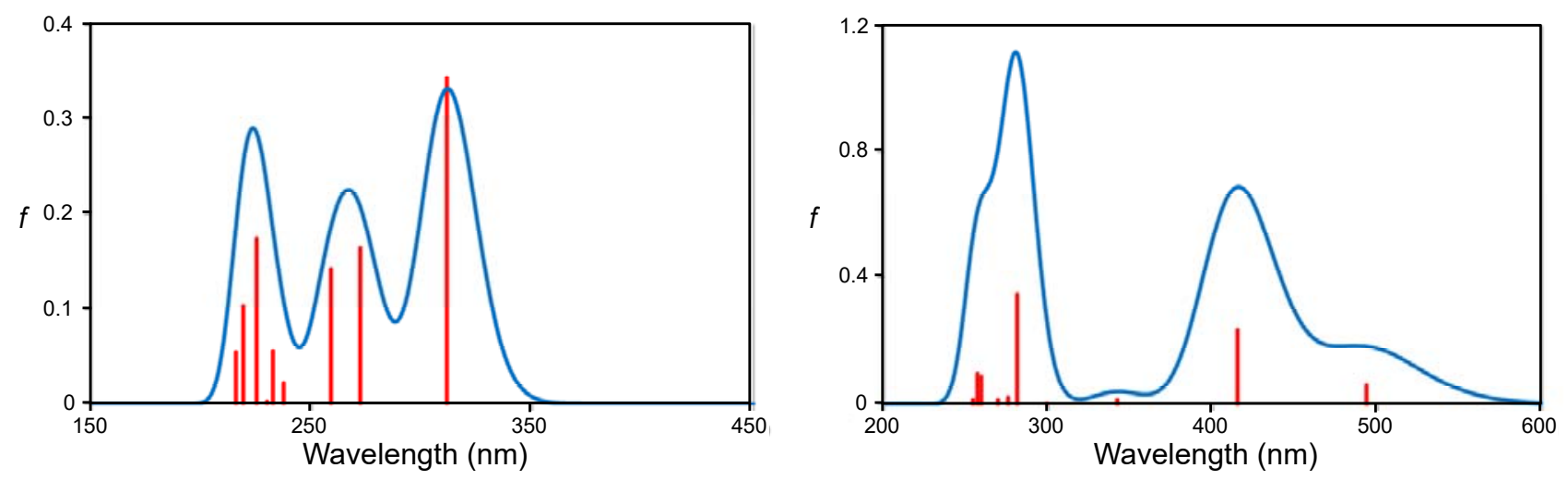

Figure S6.1H. Predicted UV-Vis spectra of $\mathbf{B N - 3}$ and BN-3a. ${ }^{19}$
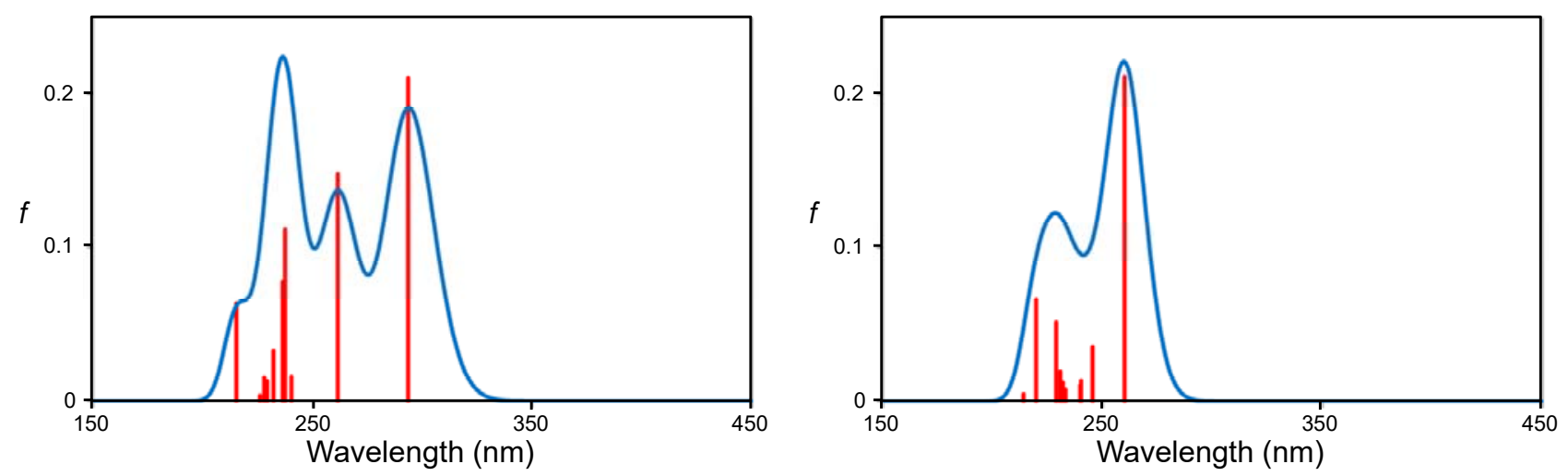

Figure S6.1I. Predicted UV-Vis spectra of $\mathbf{B N - 4}$ and $\mathbf{B N - 4 b}{ }^{19}$
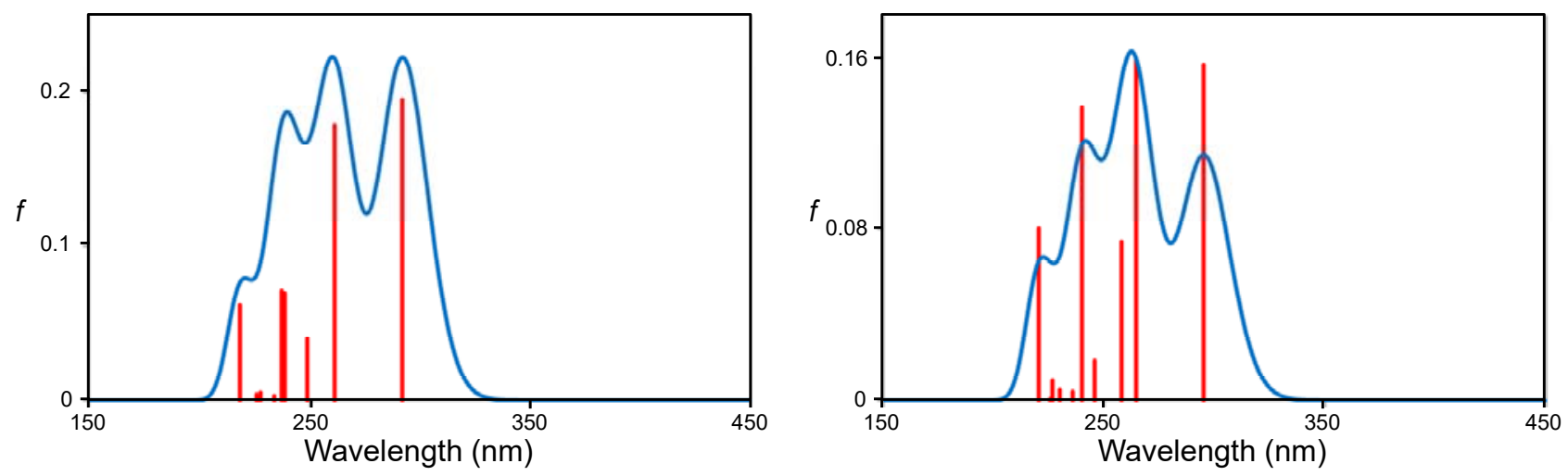

Figure S6.1J. Predicted UV-Vis spectra of $\mathbf{B N - 5}$ and BN-6. ${ }^{19}$ 


\section{X-ray Crystallographic Analysis}

The crystal data of BN-1, BN-phen1, BN-2, BN-2a, BN-4b, BN-5 and BN6 were collected on a Bruker Apex II X-ray diffractometer at $180 \mathrm{~K}$. Complete crystal structural data have been deposited at the Cambridge Crystallographic Data Centre [CCDC No. 1494632 (BN-1), 1494630 (BN-phen-1), 1494627 (BN-2), 1494629 (BN-2a), 1494631 (BN-4b), 1494628 (BN-5), 1494627 (BN-2), 1495997 (BN-6)]. These data can be obtained free of charge from The Cambridge Crystallographic Data Centre via www.ccdc.cam.ac.uk/data request/cif.

Table S7.1A. Experimental (blue) and DFT-calculated (red) bond lengths of various BN-heterocycles.

\begin{tabular}{|l|c|c|c|}
\hline & $\mathrm{B}-\mathrm{Ar}$ & $\mathrm{B}^{-\mathrm{CH}_{2}}$ & $\mathrm{~B}-\mathrm{N}$ \\
\hline BN-0 & $1.660(3) / 1.663(3) / 1.664 / 1.657$ & $1.645(3) / 1.647$ & $1.666(2) / 1.693$ \\
\hline BN-1 & $1.680(2) / 1.682(2) / 1.686 / 1.677$ & $1.639(2) / 1.647$ & $1.684(2) / 1.736$ \\
\hline BN-2 & $1.681(3) / 1.689(2) / 1.696 / 1.692$ & $1.642(2) / 1.642$ & $1.636(2) / 1.645$ \\
\hline BN-4 & $1.647(2) / 1.650(2) / 1.640 / 1.639$ & $1.617(2) / 1.626$ & $1.627(2) / 1.636$ \\
\hline BN-5 & $\mathrm{C}_{6} \mathrm{~F}_{5}: 1.661(2) / \mathrm{Ph}: 1.615(3) / \mathrm{C}_{6} \mathrm{~F}_{5}: 1.643 / \mathrm{Ph}: 1.621$ & $1.612(3) / 1.624$ & $1.635(2) / 1.651$ \\
\hline BN-6 & $1.627(2) / 1.624(2) / 1.633 / 1.629$ & $1.620(2) / 1.635$ & $1.640(2) / 1.667$ \\
\hline
\end{tabular}




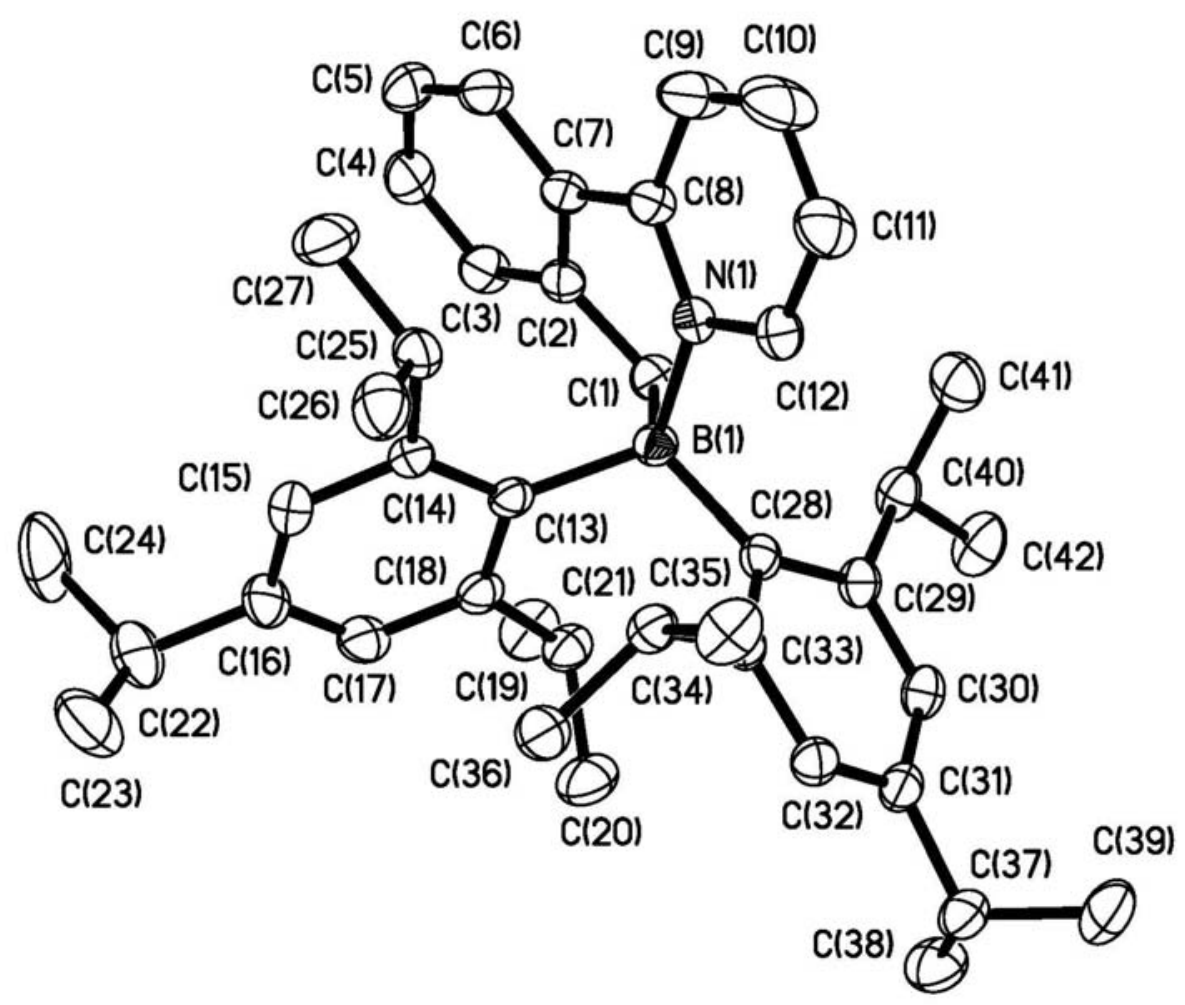

Figure S7.1. Crystal structure of BN-1 with $\mathrm{H}$ atoms omitted for clarity.

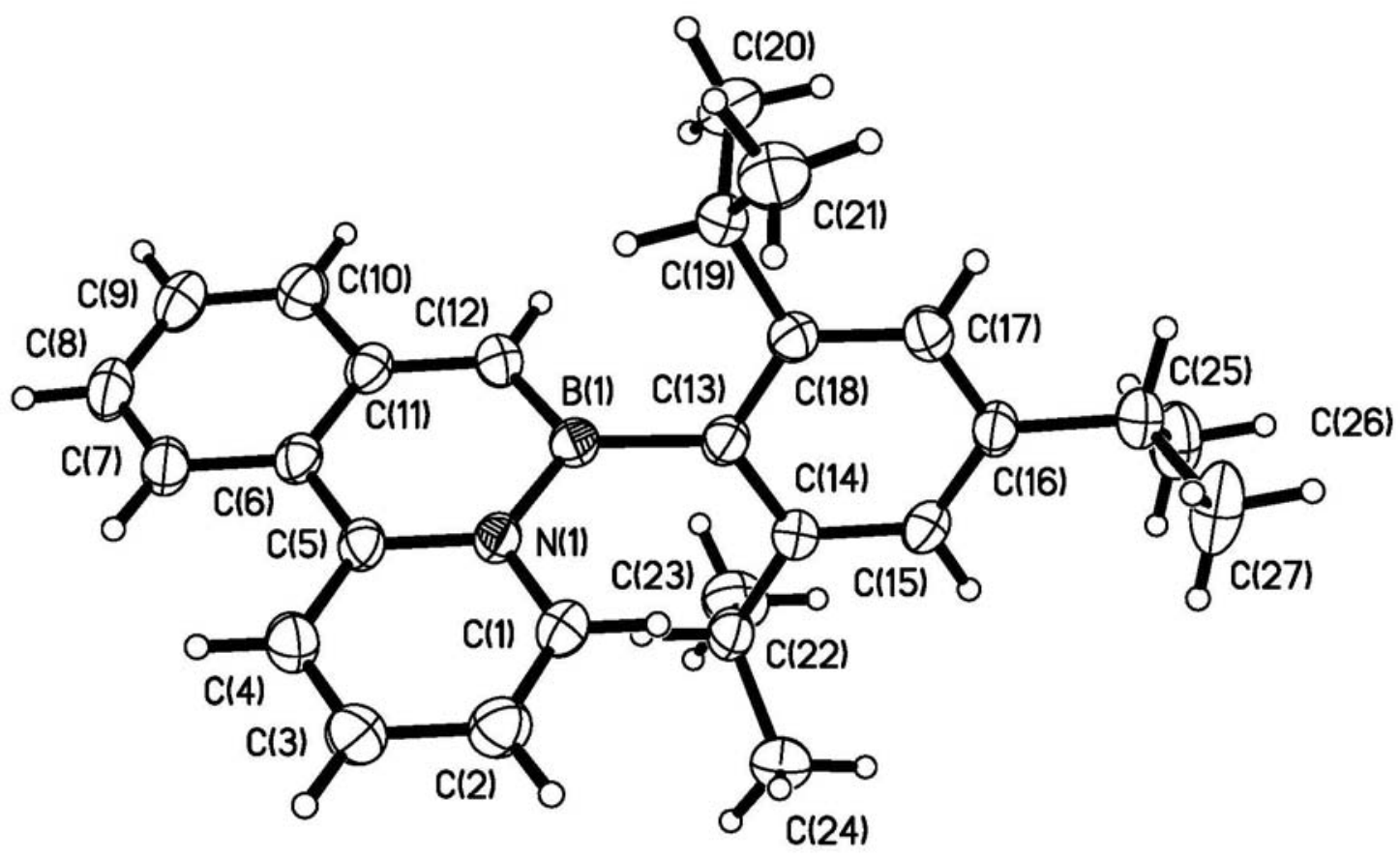

Figure S7.2. Crystal structure of BN-phen-1 


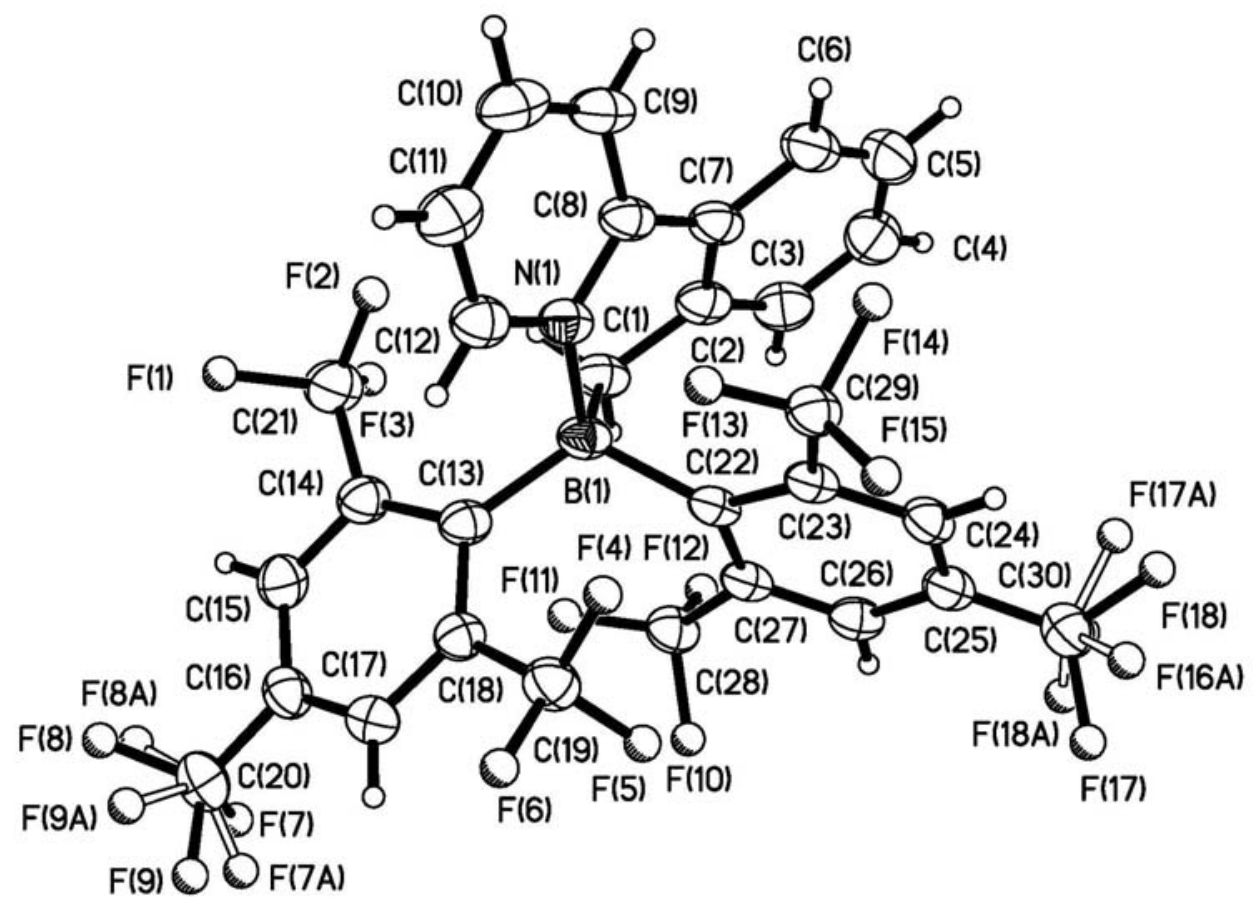

Figure S7.3. Crystal structure of $\mathbf{B N}-2$

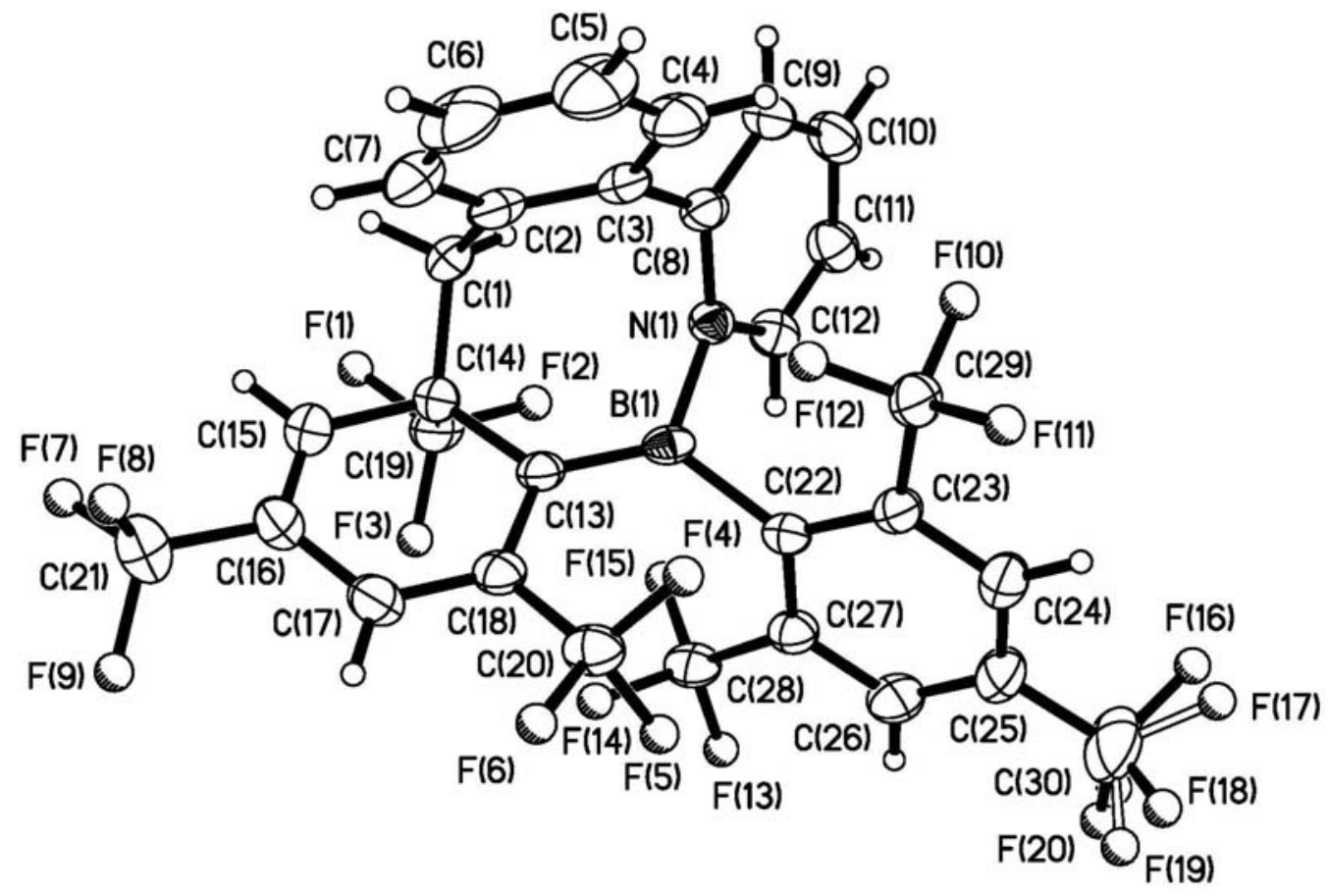

Figure S7.4. Crystal structure of BN-2a (one of the independent molecules in the asymmetric unit) 


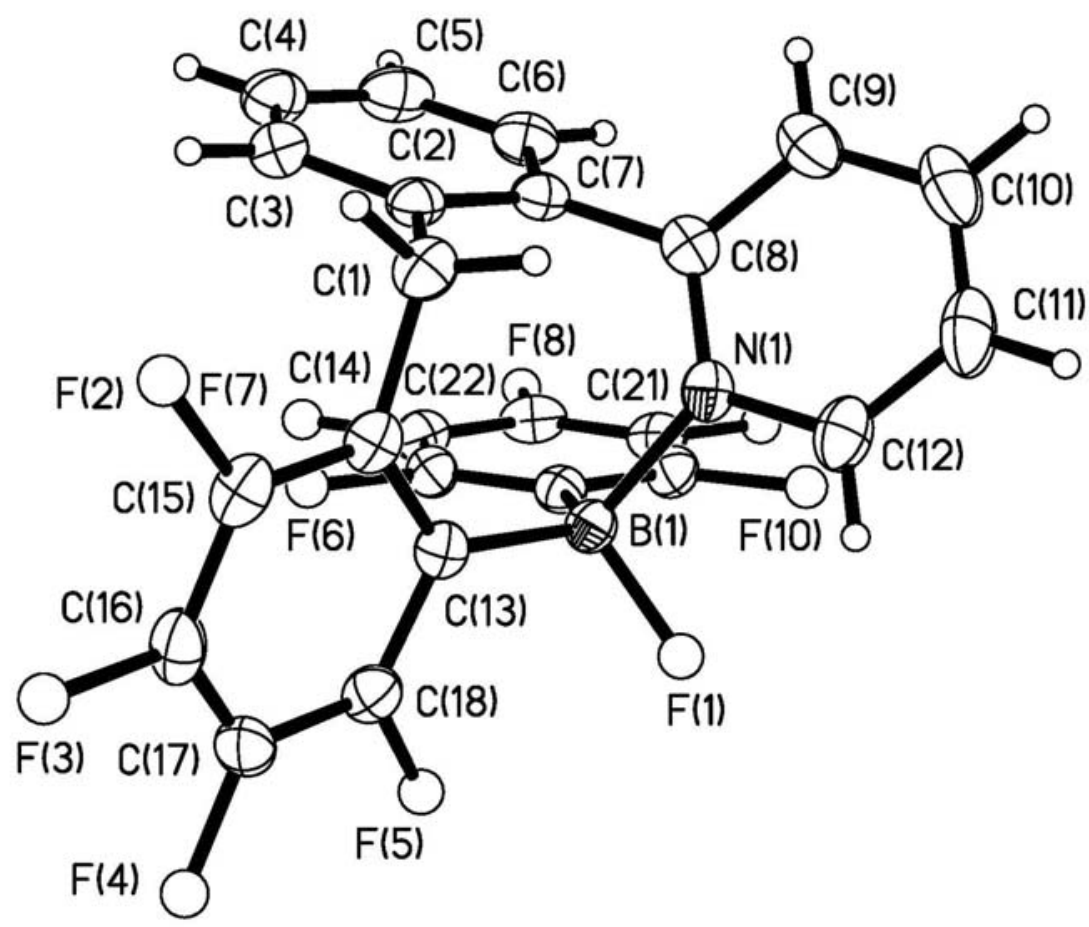

Figure S7.5. Crystal structure of BN-4b.

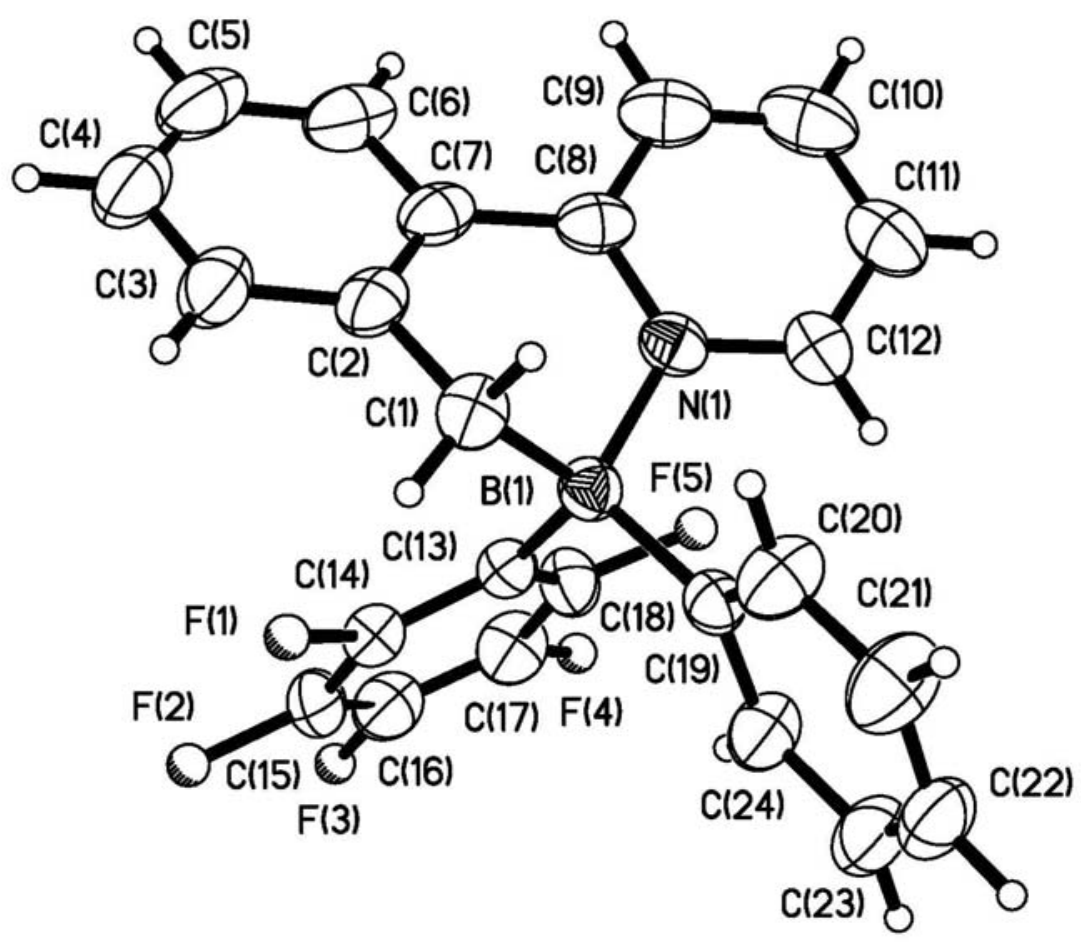

Figure S7.6. Crystal structure of BN-5 


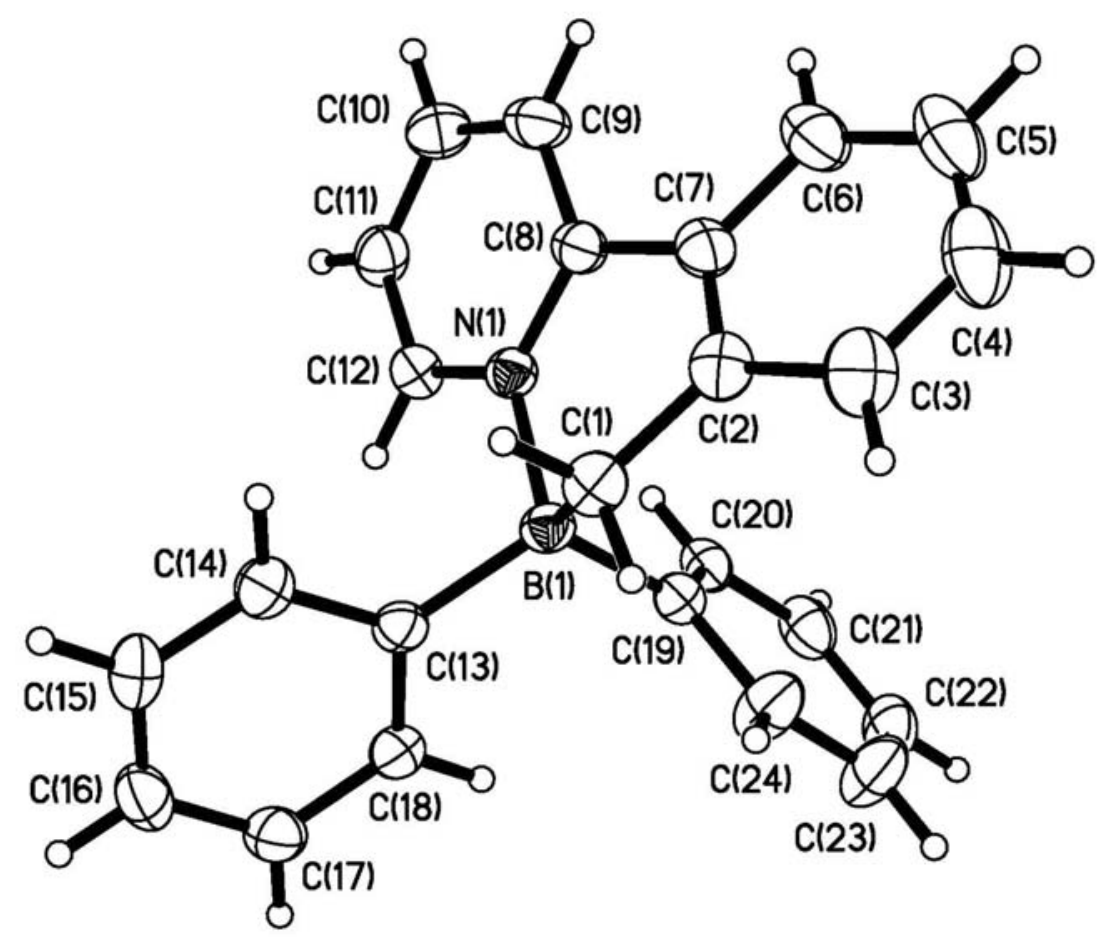

Figure S7.7. Crystal structure of BN-6 
Table 7.1B. Crystal data and structure refinement for BN-1.

Identification code

Empirical formula

Formula weight

Temperature

Wavelength

Crystal system

Space group

Unit cell dimensions

Volume

Z

Density (calculated)

Absorption coefficient

$\mathrm{F}(000)$

Crystal size

Theta range for data collection

Index ranges

Reflections collected

Independent reflections

Completeness to theta $=27.15^{\circ}$

Absorption correction

Max. and min. transmission

Refinement method

Data / restraints / parameters

Goodness-of-fit on $\mathrm{F}^{2}$

Final $\mathrm{R}$ indices [I $>2 \operatorname{sigma}(\mathrm{I})]$

$\mathrm{R}$ indices (all data)

Largest diff. peak and hole dy 37

C42 H56 B N

585.69

296(2) K

$0.71073 \AA$

Monoclinic

$\mathrm{P} 2(1) / \mathrm{c}$

$\mathrm{a}=11.2750(4) \AA$

$\alpha=90^{\circ}$.

$\mathrm{b}=18.5252(6) \AA$

$\beta=98.066(2)^{\circ}$.

$\mathrm{c}=17.7958(6) \AA$

$\gamma=90^{\circ}$.

4

$1.057 \mathrm{Mg} / \mathrm{m}^{3}$

$0.059 \mathrm{~mm}^{-1}$

1280

$0.12 \times 0.10 \times 0.08 \mathrm{~mm}^{3}$

1.59 to $27.15^{\circ}$.

$-14<=\mathrm{h}<=14,-23<=\mathrm{k}<=19,-22<=\mathrm{k}<=22$

30967

$8127[\mathrm{R}(\mathrm{int})=0.0457]$

$99.4 \%$

Semi-empirical from equivalents

0.9953 and 0.9929

Full-matrix least-squares on $\mathrm{F}^{2}$

8127 / 0 / 409

1.026

$\mathrm{R} 1=0.0509, \mathrm{wR} 2=0.1057$

$\mathrm{R} 1=0.0936, \mathrm{wR} 2=0.1237$

0.215 and -0.232 e. $\AA^{-3}$ 
Table 7.1C. Bond lengths $[\AA]$ and angles $\left[^{\circ}\right]$ for $\mathrm{BN}-1$.

\begin{tabular}{|c|c|c|c|}
\hline $\mathrm{B}(1)-\mathrm{C}(1)$ & $1.639(2)$ & $\mathrm{C}(28)-\mathrm{C}(29)$ & $1.439(2)$ \\
\hline $\mathrm{B}(1)-\mathrm{C}(13)$ & $1.680(2)$ & $C(29)-C(30)$ & $1.395(2)$ \\
\hline $\mathrm{B}(1)-\mathrm{C}(28)$ & $1.682(2)$ & $C(29)-C(40)$ & $1.530(2)$ \\
\hline $\mathrm{B}(1)-\mathrm{N}(1)$ & $1.684(2)$ & $\mathrm{C}(30)-\mathrm{C}(31)$ & $1.385(2)$ \\
\hline $\mathrm{N}(1)-\mathrm{C}(12)$ & $1.3473(18)$ & $\mathrm{C}(31)-\mathrm{C}(32)$ & $1.375(2)$ \\
\hline $\mathrm{N}(1)-\mathrm{C}(8)$ & $1.3664(19)$ & $\mathrm{C}(31)-\mathrm{C}(37)$ & $1.518(2)$ \\
\hline$C(1)-C(2)$ & $1.499(2)$ & $\mathrm{C}(32)-\mathrm{C}(33)$ & $1.402(2)$ \\
\hline $\mathrm{C}(2)-\mathrm{C}(3)$ & $1.387(2)$ & $\mathrm{C}(33)-\mathrm{C}(34)$ & $1.524(2)$ \\
\hline$C(2)-C(7)$ & $1.395(2)$ & $C(34)-C(36)$ & $1.530(2)$ \\
\hline$C(3)-C(4)$ & $1.387(2)$ & $\mathrm{C}(34)-\mathrm{C}(35)$ & $1.538(2)$ \\
\hline$C(4)-C(5)$ & $1.379(3)$ & $\mathrm{C}(37)-\mathrm{C}(39)$ & $1.524(2)$ \\
\hline$C(5)-C(6)$ & $1.375(2)$ & $\mathrm{C}(37)-\mathrm{C}(38)$ & $1.529(2)$ \\
\hline$C(6)-C(7)$ & $1.400(2)$ & $C(40)-C(42)$ & $1.525(2)$ \\
\hline$C(7)-C(8)$ & $1.469(2)$ & $\mathrm{C}(40)-\mathrm{C}(41)$ & $1.536(2)$ \\
\hline $\mathrm{C}(8)-\mathrm{C}(9)$ & $1.387(2)$ & & \\
\hline$C(9)-C(10)$ & $1.372(3)$ & $C(1)-B(1)-C(13)$ & $105.17(12)$ \\
\hline$C(10)-C(11)$ & $1.370(2)$ & $\mathrm{C}(1)-\mathrm{B}(1)-\mathrm{C}(28)$ & $120.19(12)$ \\
\hline$C(11)-C(12)$ & $1.371(2)$ & $\mathrm{C}(13)-\mathrm{B}(1)-\mathrm{C}(28)$ & $111.02(12)$ \\
\hline$C(13)-C(14)$ & $1.425(2)$ & $\mathrm{C}(1)-\mathrm{B}(1)-\mathrm{N}(1)$ & $98.59(11)$ \\
\hline$C(13)-C(18)$ & $1.430(2)$ & $\mathrm{C}(13)-\mathrm{B}(1)-\mathrm{N}(1)$ & $114.70(12)$ \\
\hline$C(14)-C(15)$ & $1.396(2)$ & $\mathrm{C}(28)-\mathrm{B}(1)-\mathrm{N}(1)$ & $106.95(11)$ \\
\hline$C(14)-C(25)$ & $1.531(2)$ & $\mathrm{C}(12)-\mathrm{N}(1)-\mathrm{C}(8)$ & $118.69(13)$ \\
\hline$C(15)-C(16)$ & $1.378(2)$ & $\mathrm{C}(12)-\mathrm{N}(1)-\mathrm{B}(1)$ & $119.43(12)$ \\
\hline$C(16)-C(17)$ & $1.378(2)$ & $\mathrm{C}(8)-\mathrm{N}(1)-\mathrm{B}(1)$ & $121.75(12)$ \\
\hline$C(16)-C(22)$ & $1.521(2)$ & $\mathrm{C}(2)-\mathrm{C}(1)-\mathrm{B}(1)$ & $110.31(12)$ \\
\hline $\mathrm{C}(17)-\mathrm{C}(18)$ & $1.398(2)$ & $C(3)-C(2)-C(7)$ & $118.47(14)$ \\
\hline $\mathrm{C}(18)-\mathrm{C}(19)$ & $1.530(2)$ & $\mathrm{C}(3)-\mathrm{C}(2)-\mathrm{C}(1)$ & $124.06(15)$ \\
\hline$C(19)-C(20)$ & $1.530(2)$ & $C(7)-C(2)-C(1)$ & $117.39(13)$ \\
\hline$C(19)-C(21)$ & $1.537(2)$ & $\mathrm{C}(4)-\mathrm{C}(3)-\mathrm{C}(2)$ & $121.28(16)$ \\
\hline$C(22)-C(23)$ & $1.518(3)$ & $C(5)-C(4)-C(3)$ & $120.00(16)$ \\
\hline$C(22)-C(24)$ & $1.523(3)$ & $C(6)-C(5)-C(4)$ & $119.70(16)$ \\
\hline$C(25)-C(26)$ & $1.525(2)$ & $C(5)-C(6)-C(7)$ & $120.66(16)$ \\
\hline$C(25)-C(27)$ & $1.536(2)$ & $C(2)-C(7)-C(6)$ & $119.87(14)$ \\
\hline C(28)-C(33) & $1.422(2)$ & $\mathrm{C}(2)-\mathrm{C}(7)-\mathrm{C}(8)$ & $119.54(14)$ \\
\hline
\end{tabular}




\begin{tabular}{|c|c|c|c|}
\hline$C(6)-C(7)-C(8)$ & $120.59(15)$ & $\mathrm{C}(16)-\mathrm{C}(22)-\mathrm{C}(24)$ & $112.11(15)$ \\
\hline $\mathrm{N}(1)-\mathrm{C}(8)-\mathrm{C}(9)$ & $119.04(14)$ & $\mathrm{C}(26)-\mathrm{C}(25)-\mathrm{C}(14)$ & $112.81(13)$ \\
\hline $\mathrm{N}(1)-\mathrm{C}(8)-\mathrm{C}(7)$ & $119.31(13)$ & $\mathrm{C}(26)-\mathrm{C}(25)-\mathrm{C}(27)$ & $108.36(13)$ \\
\hline$C(9)-C(8)-C(7)$ & $121.62(14)$ & $\mathrm{C}(14)-\mathrm{C}(25)-\mathrm{C}(27)$ & $111.21(14)$ \\
\hline $\mathrm{C}(10)-\mathrm{C}(9)-\mathrm{C}(8)$ & $121.37(17)$ & $\mathrm{C}(33)-\mathrm{C}(28)-\mathrm{C}(29)$ & $114.72(13)$ \\
\hline$C(11)-C(10)-C(9)$ & $119.06(17)$ & $\mathrm{C}(33)-\mathrm{C}(28)-\mathrm{B}(1)$ & $119.85(12)$ \\
\hline$C(10)-C(11)-C(12)$ & $118.35(16)$ & $\mathrm{C}(29)-\mathrm{C}(28)-\mathrm{B}(1)$ & $125.07(13)$ \\
\hline $\mathrm{N}(1)-\mathrm{C}(12)-\mathrm{C}(11)$ & $123.44(15)$ & $\mathrm{C}(30)-\mathrm{C}(29)-\mathrm{C}(28)$ & $120.55(14)$ \\
\hline $\mathrm{C}(14)-\mathrm{C}(13)-\mathrm{C}(18)$ & $115.34(13)$ & $C(30)-C(29)-C(40)$ & $116.94(13)$ \\
\hline $\mathrm{C}(14)-\mathrm{C}(13)-\mathrm{B}(1)$ & $129.63(13)$ & $\mathrm{C}(28)-\mathrm{C}(29)-\mathrm{C}(40)$ & $122.50(13)$ \\
\hline $\mathrm{C}(18)-\mathrm{C}(13)-\mathrm{B}(1)$ & $114.97(13)$ & $\mathrm{C}(31)-\mathrm{C}(30)-\mathrm{C}(29)$ & $123.41(14)$ \\
\hline$C(15)-C(14)-C(13)$ & $120.85(14)$ & $\mathrm{C}(32)-\mathrm{C}(31)-\mathrm{C}(30)$ & $116.17(14)$ \\
\hline$C(15)-C(14)-C(25)$ & $112.30(13)$ & $\mathrm{C}(32)-\mathrm{C}(31)-\mathrm{C}(37)$ & $121.65(15)$ \\
\hline$C(13)-C(14)-C(25)$ & $126.84(13)$ & $\mathrm{C}(30)-\mathrm{C}(31)-\mathrm{C}(37)$ & $122.18(14)$ \\
\hline$C(16)-C(15)-C(14)$ & $123.47(15)$ & $\mathrm{C}(31)-\mathrm{C}(32)-\mathrm{C}(33)$ & $122.89(15)$ \\
\hline$C(15)-C(16)-C(17)$ & $116.19(14)$ & $\mathrm{C}(32)-\mathrm{C}(33)-\mathrm{C}(28)$ & $121.39(13)$ \\
\hline$C(15)-C(16)-C(22)$ & $120.66(15)$ & $\mathrm{C}(32)-\mathrm{C}(33)-\mathrm{C}(34)$ & $114.53(13)$ \\
\hline$C(17)-C(16)-C(22)$ & $123.11(15)$ & $\mathrm{C}(28)-\mathrm{C}(33)-\mathrm{C}(34)$ & $124.07(13)$ \\
\hline$C(16)-C(17)-C(18)$ & $123.29(15)$ & $\mathrm{C}(33)-\mathrm{C}(34)-\mathrm{C}(36)$ & $112.57(13)$ \\
\hline$C(17)-C(18)-C(13)$ & $120.82(14)$ & $\mathrm{C}(33)-\mathrm{C}(34)-\mathrm{C}(35)$ & $111.00(12)$ \\
\hline $\mathrm{C}(17)-\mathrm{C}(18)-\mathrm{C}(19)$ & $114.91(14)$ & $\mathrm{C}(31)-\mathrm{C}(37)-\mathrm{C}(39)$ & $112.57(14)$ \\
\hline$C(13)-C(18)-C(19)$ & $124.24(13)$ & $\mathrm{C}(31)-\mathrm{C}(37)-\mathrm{C}(38)$ & $111.17(14)$ \\
\hline$C(18)-C(19)-C(20)$ & $111.86(14)$ & $\mathrm{C}(39)-\mathrm{C}(37)-\mathrm{C}(38)$ & $110.52(14)$ \\
\hline$C(18)-C(19)-C(21)$ & $112.46(13)$ & $C(42)-C(40)-C(29)$ & $115.14(14)$ \\
\hline$C(20)-C(19)-C(21)$ & $109.81(14)$ & $\mathrm{C}(42)-\mathrm{C}(40)-\mathrm{C}(41)$ & $108.79(14)$ \\
\hline$C(23)-C(22)-C(16)$ & $111.17(15)$ & $C(29)-C(40)-C(41)$ & 110.61(13) \\
\hline$C(23)-C(22)-C(24)$ & $109.51(17)$ & & \\
\hline
\end{tabular}


Table 7.1D. Crystal data and structure refinement for BN-phen-1.

Identification code

Empirical formula

Formula weight

Temperature

Wavelength

Crystal system

Space group

Unit cell dimensions

Volume

Z

Density (calculated)

Absorption coefficient

$\mathrm{F}(000)$

Crystal size

Theta range for data collection

Index ranges

Reflections collected

Independent reflections

Completeness to theta $=27.08^{\circ}$

Absorption correction

Max. and min. transmission

Refinement method

Data / restraints / parameters

Goodness-of-fit on $\mathrm{F}^{2}$

Final $\mathrm{R}$ indices [I $>2 \operatorname{sigma}(\mathrm{I})]$

$\mathrm{R}$ indices (all data)

Extinction coefficient

Largest diff. peak and hole dy 40

C27 H32 B N

381.35

180(2) K

$0.71073 \AA$

Orthorhombic

Pbcn

$\mathrm{a}=31.316(4) \AA \quad \alpha=90^{\circ}$.

$\mathrm{b}=8.2742(12) \AA \quad \beta=90^{\circ}$.

$\mathrm{c}=16.832(2) \AA \quad \gamma=90^{\circ}$.

4361.4(11) $\AA^{3}$

8

$1.162 \mathrm{Mg} / \mathrm{m}^{3}$

$0.065 \mathrm{~mm}^{-1}$

1648

$0.15 \times 0.12 \times 0.05 \mathrm{~mm}^{3}$

2.42 to $27.08^{\circ}$.

$-40<=\mathrm{h}<=39,-10<=\mathrm{k}<=9,-14<=1<=21$

20262

$4770[\mathrm{R}(\mathrm{int})=0.0821]$

$99.2 \%$

Semi-empirical from equivalents

0.9967 and 0.9903

Full-matrix least-squares on $\mathrm{F}^{2}$

4770 / 0 / 269

1.007

$\mathrm{R} 1=0.0513, \mathrm{wR} 2=0.1045$

$\mathrm{R} 1=0.1111, \mathrm{wR} 2=0.1276$

$0.0039(5)$

0.227 and -0.171 e. $\AA^{-3}$ 
Table 7.1E. Bond lengths $[\AA]$ and angles $\left[{ }^{\circ}\right]$ for BN-phen-1.

\begin{tabular}{|c|c|c|c|}
\hline $\mathrm{N}(1)-\mathrm{C}(1)$ & $1.384(2)$ & $\mathrm{C}(1)-\mathrm{N}(1)-\mathrm{B}(1)$ & $118.90(14)$ \\
\hline $\mathrm{N}(1)-\mathrm{C}(5)$ & $1.395(2)$ & $\mathrm{C}(5)-\mathrm{N}(1)-\mathrm{B}(1)$ & $122.85(14)$ \\
\hline $\mathrm{N}(1)-\mathrm{B}(1)$ & $1.495(2)$ & $\mathrm{C}(12)-\mathrm{B}(1)-\mathrm{N}(1)$ & $114.35(15)$ \\
\hline $\mathrm{B}(1)-\mathrm{C}(12)$ & $1.463(3)$ & $\mathrm{C}(12)-\mathrm{B}(1)-\mathrm{C}(13)$ & $125.77(16)$ \\
\hline $\mathrm{B}(1)-\mathrm{C}(13)$ & $1.590(3)$ & $\mathrm{N}(1)-\mathrm{B}(1)-\mathrm{C}(13)$ & $119.88(16)$ \\
\hline $\mathrm{C}(1)-\mathrm{C}(2)$ & $1.347(2)$ & $\mathrm{C}(2)-\mathrm{C}(1)-\mathrm{N}(1)$ & $123.67(17)$ \\
\hline $\mathrm{C}(2)-\mathrm{C}(3)$ & $1.400(3)$ & $\mathrm{C}(1)-\mathrm{C}(2)-\mathrm{C}(3)$ & $119.07(18)$ \\
\hline$C(3)-C(4)$ & $1.356(3)$ & $\mathrm{C}(4)-\mathrm{C}(3)-\mathrm{C}(2)$ & $118.85(17)$ \\
\hline$C(4)-C(5)$ & $1.418(2)$ & $C(3)-C(4)-C(5)$ & $122.46(17)$ \\
\hline$C(5)-C(6)$ & $1.430(2)$ & $\mathrm{N}(1)-\mathrm{C}(5)-\mathrm{C}(4)$ & $117.69(16)$ \\
\hline$C(6)-C(7)$ & $1.420(2)$ & $\mathrm{N}(1)-\mathrm{C}(5)-\mathrm{C}(6)$ & $119.59(15)$ \\
\hline $\mathrm{C}(6)-\mathrm{C}(11)$ & $1.429(2)$ & $C(4)-C(5)-C(6)$ & $122.72(16)$ \\
\hline$C(7)-C(8)$ & $1.357(3)$ & $C(7)-C(6)-C(11)$ & $118.40(16)$ \\
\hline$C(8)-C(9)$ & $1.404(3)$ & $C(7)-C(6)-C(5)$ & $121.25(16)$ \\
\hline $\mathrm{C}(9)-\mathrm{C}(10)$ & $1.353(2)$ & $C(11)-C(6)-C(5)$ & $120.34(15)$ \\
\hline $\mathrm{C}(10)-\mathrm{C}(11)$ & $1.427(2)$ & $\mathrm{C}(8)-\mathrm{C}(7)-\mathrm{C}(6)$ & $121.76(17)$ \\
\hline $\mathrm{C}(11)-\mathrm{C}(12)$ & $1.403(2)$ & $\mathrm{C}(7)-\mathrm{C}(8)-\mathrm{C}(9)$ & $120.19(18)$ \\
\hline $\mathrm{C}(13)-\mathrm{C}(18)$ & $1.402(3)$ & $\mathrm{C}(10)-\mathrm{C}(9)-\mathrm{C}(8)$ & $119.88(18)$ \\
\hline$C(13)-C(14)$ & $1.414(2)$ & $\mathrm{C}(9)-\mathrm{C}(10)-\mathrm{C}(11)$ & $122.32(17)$ \\
\hline $\mathrm{C}(14)-\mathrm{C}(15)$ & $1.398(2)$ & $\mathrm{C}(12)-\mathrm{C}(11)-\mathrm{C}(10)$ & $122.34(16)$ \\
\hline$C(14)-C(22)$ & $1.515(3)$ & $C(12)-C(11)-C(6)$ & $120.28(16)$ \\
\hline$C(15)-C(16)$ & $1.381(3)$ & $\mathrm{C}(10)-\mathrm{C}(11)-\mathrm{C}(6)$ & $117.38(16)$ \\
\hline$C(16)-C(17)$ & $1.384(2)$ & $\mathrm{C}(11)-\mathrm{C}(12)-\mathrm{B}(1)$ & $122.57(16)$ \\
\hline$C(16)-C(25)$ & $1.522(2)$ & $\mathrm{C}(18)-\mathrm{C}(13)-\mathrm{C}(14)$ & $118.17(15)$ \\
\hline$C(17)-C(18)$ & $1.396(2)$ & $\mathrm{C}(18)-\mathrm{C}(13)-\mathrm{B}(1)$ & $121.07(14)$ \\
\hline$C(18)-C(19)$ & $1.525(2)$ & $\mathrm{C}(14)-\mathrm{C}(13)-\mathrm{B}(1)$ & $120.59(17)$ \\
\hline$C(19)-C(21)$ & $1.516(3)$ & $C(15)-C(14)-C(13)$ & $119.70(18)$ \\
\hline$C(19)-C(20)$ & $1.529(2)$ & $C(15)-C(14)-C(22)$ & $119.36(16)$ \\
\hline$C(22)-C(23)$ & $1.524(3)$ & $\mathrm{C}(13)-\mathrm{C}(14)-\mathrm{C}(22)$ & $120.76(15)$ \\
\hline$C(22)-C(24)$ & $1.525(2)$ & $C(16)-C(15)-C(14)$ & $122.36(16)$ \\
\hline$C(25)-C(26)$ & $1.508(3)$ & $\mathrm{C}(15)-\mathrm{C}(16)-\mathrm{C}(17)$ & $117.39(16)$ \\
\hline \multirow[t]{2}{*}{$C(25)-C(27)$} & $1.507(3)$ & $\mathrm{C}(15)-\mathrm{C}(16)-\mathrm{C}(25)$ & $122.56(16)$ \\
\hline & & $\mathrm{C}(17)-\mathrm{C}(16)-\mathrm{C}(25)$ & $120.05(18)$ \\
\hline $\mathrm{C}(1)-\mathrm{N}(1)-\mathrm{C}(5)$ & $118.24(14)$ & $\mathrm{C}(16)-\mathrm{C}(17)-\mathrm{C}(18)$ & $122.45(18)$ \\
\hline
\end{tabular}




$\begin{array}{llll}\mathrm{C}(17)-\mathrm{C}(18)-\mathrm{C}(13) & 119.92(16) & \mathrm{C}(14)-\mathrm{C}(22)-\mathrm{C}(23) & 110.04(15) \\ \mathrm{C}(17)-\mathrm{C}(18)-\mathrm{C}(19) & 118.51(17) & \mathrm{C}(14)-\mathrm{C}(22)-\mathrm{C}(24) & 113.58(17) \\ \mathrm{C}(13)-\mathrm{C}(18)-\mathrm{C}(19) & 121.50(15) & \mathrm{C}(23)-\mathrm{C}(22)-\mathrm{C}(24) & 110.65(17) \\ \mathrm{C}(21)-\mathrm{C}(19)-\mathrm{C}(18) & 113.41(15) & \mathrm{C}(26)-\mathrm{C}(25)-\mathrm{C}(27) & 110.75(16) \\ \mathrm{C}(21)-\mathrm{C}(19)-\mathrm{C}(20) & 110.07(17) & \mathrm{C}(26)-\mathrm{C}(25)-\mathrm{C}(16) & 113.00(18) \\ \mathrm{C}(18)-\mathrm{C}(19)-\mathrm{C}(20) & 109.84(15) & \mathrm{C}(27)-\mathrm{C}(25)-\mathrm{C}(16) & 111.82(16)\end{array}$


Table 7.1F. Crystal data and structure refinement for BN-2.

Identification code

Empirical formula

Formula weight

Temperature

Wavelength

Crystal system

Space group

Unit cell dimensions

Volume

Z

Density (calculated)

Absorption coefficient

$\mathrm{F}(000)$

Crystal size

Theta range for data collection

Index ranges

Reflections collected

Independent reflections

Completeness to theta $=27.15^{\circ}$

Absorption correction

Max. and min. transmission

Refinement method

Data / restraints / parameters

Goodness-of-fit on $\mathrm{F}^{2}$

Final $\mathrm{R}$ indices [I $>2 \operatorname{sigma}(\mathrm{I})]$

$\mathrm{R}$ indices (all data)

Largest diff. peak and hole jb4

C30 H14 B F18 N

741.23

296(2) K

$0.71073 \AA$

Triclinic

P-1

$\mathrm{a}=10.8184(6) \AA$

$\alpha=76.622(3)^{\circ}$.

$\mathrm{b}=11.8123(6) \AA$

$\beta=67.526(3)^{\circ}$.

$\mathrm{c}=12.9256(6) \AA$

$\gamma=70.094(3)^{\circ}$.

1425.35(13) $\AA^{3}$

2

$1.727 \mathrm{Mg} / \mathrm{m}^{3}$

$0.183 \mathrm{~mm}^{-1}$

736

$0.20 \times 0.15 \times 0.05 \mathrm{~mm}^{3}$

1.72 to $27.15^{\circ}$.

$-13<=\mathrm{h}<=12,-15<=\mathrm{k}<=15,-16<=\mathrm{k}<=16$

23859

$6275[\mathrm{R}(\mathrm{int})=0.0293]$

$99.2 \%$

Semi-empirical from equivalents

0.9909 and 0.9642

Full-matrix least-squares on $\mathrm{F}^{2}$

6275 / 0 / 505

1.041

$\mathrm{R} 1=0.0393, \mathrm{wR} 2=0.0972$

$\mathrm{R} 1=0.0546, \mathrm{wR} 2=0.1070$

0.265 and -0.239 e. $\AA^{-3}$ 
Table 7.1G. Bond lengths $[\AA]$ and angles $\left[^{\circ}\right]$ for BN-2.

\begin{tabular}{|c|c|c|c|}
\hline $\mathrm{B}(1)-\mathrm{N}(1)$ & $1.636(2)$ & $C(4)-C(5)$ & $1.381(3)$ \\
\hline $\mathrm{B}(1)-\mathrm{C}(1)$ & $1.642(2)$ & $C(5)-C(6)$ & $1.376(3)$ \\
\hline $\mathrm{B}(1)-\mathrm{C}(13)$ & $1.681(3)$ & $C(6)-C(7)$ & $1.395(3)$ \\
\hline $\mathrm{B}(1)-\mathrm{C}(22)$ & $1.689(2)$ & $\mathrm{C}(7)-\mathrm{C}(8)$ & $1.470(3)$ \\
\hline $\mathrm{N}(1)-\mathrm{C}(12)$ & $1.346(2)$ & $\mathrm{C}(8)-\mathrm{C}(9)$ & $1.392(2)$ \\
\hline $\mathrm{N}(1)-\mathrm{C}(8)$ & $1.370(2)$ & $C(9)-C(10)$ & $1.371(3)$ \\
\hline $\mathrm{F}(1)-\mathrm{C}(21)$ & $1.347(2)$ & $\mathrm{C}(10)-\mathrm{C}(11)$ & $1.377(3)$ \\
\hline $\mathrm{F}(2)-\mathrm{C}(21)$ & $1.336(2)$ & $\mathrm{C}(11)-\mathrm{C}(12)$ & $1.375(2)$ \\
\hline $\mathrm{F}(3)-\mathrm{C}(21)$ & $1.333(3)$ & $\mathrm{C}(13)-\mathrm{C}(18)$ & $1.424(2)$ \\
\hline $\mathrm{F}(4)-\mathrm{C}(19)$ & $1.332(2)$ & $C(13)-C(14)$ & $1.430(2)$ \\
\hline $\mathrm{F}(5)-\mathrm{C}(19)$ & $1.344(2)$ & $C(14)-C(15)$ & $1.387(3)$ \\
\hline $\mathrm{F}(6)-\mathrm{C}(19)$ & $1.340(2)$ & $C(14)-C(21)$ & $1.514(2)$ \\
\hline $\mathrm{F}(7)-\mathrm{C}(20)$ & $1.329(3)$ & $C(15)-C(16)$ & $1.376(2)$ \\
\hline $\mathrm{F}(8)-\mathrm{C}(20)$ & $1.312(3)$ & $C(16)-C(17)$ & $1.375(2)$ \\
\hline $\mathrm{F}(9)-\mathrm{C}(20)$ & $1.308(3)$ & $C(16)-C(20)$ & $1.496(3)$ \\
\hline $\mathrm{F}(7 \mathrm{~A})-\mathrm{C}(20)$ & $1.180(8)$ & $C(17)-C(18)$ & $1.389(2)$ \\
\hline $\mathrm{F}(8 \mathrm{~A})-\mathrm{C}(20)$ & $1.243(12)$ & $C(18)-C(19)$ & $1.510(2)$ \\
\hline $\mathrm{F}(9 \mathrm{~A})-\mathrm{C}(20)$ & $1.313(9)$ & $C(22)-C(23)$ & $1.427(2)$ \\
\hline $\mathrm{F}(10)-\mathrm{C}(28)$ & $1.3412(19)$ & $C(22)-C(27)$ & $1.430(2)$ \\
\hline $\mathrm{F}(11)-\mathrm{C}(28)$ & $1.326(2)$ & $C(23)-C(24)$ & $1.397(2)$ \\
\hline $\mathrm{F}(12)-\mathrm{C}(28)$ & $1.348(2)$ & $\mathrm{C}(23)-\mathrm{C}(29)$ & $1.516(2)$ \\
\hline $\mathrm{F}(13)-\mathrm{C}(29)$ & $1.314(2)$ & $C(24)-C(25)$ & $1.371(2)$ \\
\hline $\mathrm{F}(14)-\mathrm{C}(29)$ & $1.334(2)$ & $C(25)-C(26)$ & $1.375(2)$ \\
\hline $\mathrm{F}(15)-\mathrm{C}(29)$ & $1.3431(18)$ & $C(25)-C(30)$ & $1.492(3)$ \\
\hline $\mathrm{F}(16)-\mathrm{C}(30)$ & $1.348(3)$ & $C(26)-C(27)$ & $1.387(2)$ \\
\hline $\mathrm{F}(17)-\mathrm{C}(30)$ & $1.340(2)$ & $\mathrm{C}(27)-\mathrm{C}(28)$ & $1.519(2)$ \\
\hline $\mathrm{F}(18)-\mathrm{C}(30)$ & $1.318(3)$ & & \\
\hline $\mathrm{F}(16 \mathrm{~A})-\mathrm{C}(30)$ & $1.288(7)$ & $\mathrm{N}(1)-\mathrm{B}(1)-\mathrm{C}(1)$ & $101.15(12)$ \\
\hline $\mathrm{F}(17 \mathrm{~A})-\mathrm{C}(30)$ & $1.327(9)$ & $\mathrm{N}(1)-\mathrm{B}(1)-\mathrm{C}(13)$ & $107.81(14)$ \\
\hline $\mathrm{F}(18 \mathrm{~A})-\mathrm{C}(30)$ & $1.178(7)$ & $\mathrm{C}(1)-\mathrm{B}(1)-\mathrm{C}(13)$ & $115.58(13)$ \\
\hline$C(1)-C(2)$ & $1.501(2)$ & $\mathrm{N}(1)-\mathrm{B}(1)-\mathrm{C}(22)$ & $114.18(13)$ \\
\hline$C(2)-C(3)$ & $1.382(3)$ & $\mathrm{C}(1)-\mathrm{B}(1)-\mathrm{C}(22)$ & $101.34(14)$ \\
\hline $\mathrm{C}(2)-\mathrm{C}(7)$ & $1.399(2)$ & $\mathrm{C}(13)-\mathrm{B}(1)-\mathrm{C}(22)$ & $115.91(12)$ \\
\hline$C(3)-C(4)$ & $1.384(3)$ & $\mathrm{C}(12)-\mathrm{N}(1)-\mathrm{C}(8)$ & $118.90(14)$ \\
\hline
\end{tabular}




\begin{tabular}{|c|c|}
\hline $\mathrm{C}(12)-\mathrm{N}(1)-\mathrm{B}(1)$ & $120.18(14)$ \\
\hline $\mathrm{C}(8)-\mathrm{N}(1)-\mathrm{B}(1)$ & $120.76(14)$ \\
\hline $\mathrm{C}(2)-\mathrm{C}(1)-\mathrm{B}(1)$ & $108.45(13)$ \\
\hline$C(3)-C(2)-C(7)$ & $118.92(17)$ \\
\hline$C(3)-C(2)-C(1)$ & $123.74(16)$ \\
\hline$C(7)-C(2)-C(1)$ & $117.35(16)$ \\
\hline$C(2)-C(3)-C(4)$ & $120.93(19)$ \\
\hline$C(5)-C(4)-C(3)$ & $120.1(2)$ \\
\hline$C(6)-C(5)-C(4)$ & $119.85(19)$ \\
\hline$C(5)-C(6)-C(7)$ & $120.46(18)$ \\
\hline$C(6)-C(7)-C(2)$ & 119.71(18) \\
\hline$C(6)-C(7)-C(8)$ & $120.62(16)$ \\
\hline$C(2)-C(7)-C(8)$ & $119.66(16)$ \\
\hline $\mathrm{N}(1)-\mathrm{C}(8)-\mathrm{C}(9)$ & $119.24(17)$ \\
\hline $\mathrm{N}(1)-\mathrm{C}(8)-\mathrm{C}(7)$ & $118.79(14)$ \\
\hline $\mathrm{C}(9)-\mathrm{C}(8)-\mathrm{C}(7)$ & $121.96(16)$ \\
\hline $\mathrm{C}(10)-\mathrm{C}(9)-\mathrm{C}(8)$ & 121.11(17) \\
\hline$C(9)-C(10)-C(11)$ & 119.11(17) \\
\hline $\mathrm{C}(12)-\mathrm{C}(11)-\mathrm{C}(10)$ & $118.54(19)$ \\
\hline $\mathrm{N}(1)-\mathrm{C}(12)-\mathrm{C}(11)$ & $123.09(17)$ \\
\hline$C(18)-C(13)-C(14)$ & $111.86(15)$ \\
\hline $\mathrm{C}(18)-\mathrm{C}(13)-\mathrm{B}(1)$ & $124.31(14)$ \\
\hline $\mathrm{C}(14)-\mathrm{C}(13)-\mathrm{B}(1)$ & $123.26(14)$ \\
\hline $\mathrm{C}(15)-\mathrm{C}(14)-\mathrm{C}(13)$ & $123.36(15)$ \\
\hline $\mathrm{C}(15)-\mathrm{C}(14)-\mathrm{C}(21)$ & $111.34(15)$ \\
\hline $\mathrm{C}(13)-\mathrm{C}(14)-\mathrm{C}(21)$ & $125.30(16)$ \\
\hline$C(16)-C(15)-C(14)$ & $120.97(16)$ \\
\hline$C(17)-C(16)-C(15)$ & $118.13(16)$ \\
\hline$C(17)-C(16)-C(20)$ & $121.03(16)$ \\
\hline$C(15)-C(16)-C(20)$ & $120.81(16)$ \\
\hline$C(16)-C(17)-C(18)$ & $120.78(15)$ \\
\hline $\mathrm{C}(17)-\mathrm{C}(18)-\mathrm{C}(13)$ & $123.70(15)$ \\
\hline $\mathrm{C}(17)-\mathrm{C}(18)-\mathrm{C}(19)$ & $112.18(14)$ \\
\hline$C(13)-C(18)-C(19)$ & $124.12(15)$ \\
\hline $\mathrm{F}(4)-\mathrm{C}(19)-\mathrm{F}(6)$ & $105.57(14)$ \\
\hline $\mathrm{F}(4)-\mathrm{C}(19)-\mathrm{F}(5)$ & $107.35(14)$ \\
\hline
\end{tabular}

$\begin{array}{ll}F(6)-C(19)-F(5) & 105.62(14) \\ F(4)-C(19)-C(18) & 113.40(14) \\ F(6)-C(19)-C(18) & 111.22(15) \\ F(5)-C(19)-C(18) & 113.10(14) \\ F(7 A)-C(20)-F(8 A) & 113.4(14) \\ F(8 A)-C(20)-F(9) & 128.6(8) \\ F(7 A)-C(20)-F(8) & 129.1(7) \\ F(9)-C(20)-F(8) & 108.2(3) \\ F(7 A)-C(20)-F(9 A) & 105.1(14) \\ F(8 A)-C(20)-F(9 A) & 98.9(11) \\ F(9)-C(20)-F(7) & 105.0(2) \\ F(8)-C(20)-F(7) & 104.1(2) \\ F(7 A)-C(20)-C(16) & 116.9(5) \\ F(8 A)-C(20)-C(16) & 113.6(8) \\ F(9)-C(20)-C(16) & 114.11(18) \\ F(8)-C(20)-C(16) & 113.2(2) \\ F(9 A)-C(20)-C(16) & 106.8(4) \\ F(7)-C(20)-C(16) & 111.39(18) \\ F(3)-C(21)-F(2) & 107.30(17) \\ F(3)-C(21)-F(1) & 105.68(16) \\ F(2)-C(21)-F(1) & 104.87(17) \\ F(3)-C(21)-C(14) & 113.31(17) \\ F(2)-C(21)-C(14) & 114.40(15) \\ F(1)-C(21)-C(14) & 110.59(17) \\ C(23)-C(22)-C(27) & 112.34(14) \\ C(23)-C(22)-B(1) & 128.24(13) \\ C(27)-C(22)-B(1) & 118.35(13) \\ C(24)-C(23)-C(22) & 122.91(15) \\ C(24)-C(23)-C(29) & 108.36(14) \\ C(22)-C(23)-C(29) & 128.73(15) \\ C(25)-C(24)-C(23) & 121.47(15) \\ C(24)-C(25)-C(26) & 118.19(16) \\ C(24)-C(25)-C(30) & 127(15) \\ C(26)-C(27)-C(22) & \\ & \end{array}$




$\begin{array}{llll}\mathrm{C}(26)-\mathrm{C}(27)-\mathrm{C}(28) & 108.19(14) & \mathrm{F}(18 \mathrm{~A})-\mathrm{C}(30)-\mathrm{F}(16 \mathrm{~A}) & 118.8(8) \\ \mathrm{C}(22)-\mathrm{C}(27)-\mathrm{C}(28) & 128.08(15) & \mathrm{F}(18 \mathrm{~A})-\mathrm{C}(30)-\mathrm{F}(18) & 131.6(5) \\ \mathrm{F}(11)-\mathrm{C}(28)-\mathrm{F}(10) & 105.73(14) & \mathrm{F}(18 \mathrm{~A})-\mathrm{C}(30)-\mathrm{F}(17 \mathrm{~A}) & 108.3(8) \\ \mathrm{F}(11)-\mathrm{C}(28)-\mathrm{F}(12) & 105.77(14) & \mathrm{F}(16 \mathrm{~A})-\mathrm{C}(30)-\mathrm{F}(17 \mathrm{~A}) & 106.9(7) \\ \mathrm{F}(10)-\mathrm{C}(28)-\mathrm{F}(12) & 105.46(14) & \mathrm{F}(18)-\mathrm{C}(30)-\mathrm{F}(17) & 106.3(2) \\ \mathrm{F}(11)-\mathrm{C}(28)-\mathrm{C}(27) & 118.16(14) & \mathrm{F}(18)-\mathrm{C}(30)-\mathrm{F}(16) & 105.4(2) \\ \mathrm{F}(10)-\mathrm{C}(28)-\mathrm{C}(27) & 110.11(14) & \mathrm{F}(17)-\mathrm{C}(30)-\mathrm{F}(16) & 102.79(18) \\ \mathrm{F}(12)-\mathrm{C}(28)-\mathrm{C}(27) & 110.75(14) & \mathrm{F}(18 \mathrm{~A})-\mathrm{C}(30)-\mathrm{C}(25) & 111.5(4) \\ \mathrm{F}(13)-\mathrm{C}(29)-\mathrm{F}(14) & 105.96(13) & \mathrm{F}(16 \mathrm{~A})-\mathrm{C}(30)-\mathrm{C}(25) & 108.3(3) \\ \mathrm{F}(13)-\mathrm{C}(29)-\mathrm{F}(15) & 104.01(14) & \mathrm{F}(18)-\mathrm{C}(30)-\mathrm{C}(25) & 115.03(16) \\ \mathrm{F}(14)-\mathrm{C}(29)-\mathrm{F}(15) & 105.70(14) & \mathrm{F}(17 \mathrm{~A})-\mathrm{C}(30)-\mathrm{C}(25) & 101.6(4) \\ \mathrm{F}(13)-\mathrm{C}(29)-\mathrm{C}(23) & 118.81(14) & \mathrm{F}(17)-\mathrm{C}(30)-\mathrm{C}(25) & 112.81(16) \\ \mathrm{F}(14)-\mathrm{C}(29)-\mathrm{C}(23) & 111.40(14) & \mathrm{F}(16)-\mathrm{C}(30)-\mathrm{C}(25) & 113.47(17) \\ \mathrm{F}(15)-\mathrm{C}(29)-\mathrm{C}(23) & 109.98(13) & & \end{array}$


Table 7.1H. Crystal data and structure refinement for BN-2a.

Identification code

Empirical formula

Formula weight

Temperature

Wavelength

Crystal system

Space group

Unit cell dimensions

Volume

Z

Density (calculated)

Absorption coefficient

$\mathrm{F}(000)$

Crystal size

Theta range for data collection

Index ranges

Reflections collected

Independent reflections

Completeness to theta $=27.27^{\circ}$

Absorption correction

Max. and min. transmission

Refinement method

Data / restraints / parameters

Goodness-of-fit on $\mathrm{F}^{2}$

Final $\mathrm{R}$ indices [I $>2 \operatorname{sigma}(\mathrm{I})]$

$\mathrm{R}$ indices (all data)

Largest diff. peak and hole dy 41

C60 H28 B2 F36 N2

1482.46

180(2) K

$0.71073 \AA$

Triclinic

P-1

$\mathrm{a}=8.8041(2) \AA$

$\alpha=78.7090(10)^{\circ}$.

$\mathrm{b}=16.8557(3) \AA$

$\beta=79.5870(10)^{\circ}$.

$\mathrm{c}=20.7579(4) \AA$

$\gamma=82.4680(10)^{\circ}$.

2956.43(10) $\AA^{3}$

2

$1.665 \mathrm{Mg} / \mathrm{m}^{3}$

$0.177 \mathrm{~mm}^{-1}$

1472

$0.10 \times 0.10 \times 0.06 \mathrm{~mm}^{3}$

1.73 to $27.27^{\circ}$.

$-11<=\mathrm{h}<=11,-21<=\mathrm{k}<=21,-25<=\mathrm{l}<=26$

40608

$13173[\mathrm{R}(\mathrm{int})=0.0477]$

$99.1 \%$

Semi-empirical from equivalents

0.9895 and 0.9825

Full-matrix least-squares on $\mathrm{F}^{2}$

$13173 / 12$ / 955

0.929

$\mathrm{R} 1=0.0437, \mathrm{wR} 2=0.0932$

$\mathrm{R} 1=0.0967, \mathrm{wR} 2=0.1088$

0.228 and -0.342 e. $\AA^{-3}$ 
Table 7.1I. Bond lengths $[\AA]$ and angles $\left[{ }^{\circ}\right]$ for BN-2a.

\begin{tabular}{|c|c|c|c|}
\hline $\mathrm{B}(1)-\mathrm{C}(13)$ & $1.465(3)$ & $\mathrm{C}(19)-\mathrm{F}(1)$ & $1.349(2)$ \\
\hline $\mathrm{B}(1)-\mathrm{N}(1)$ & $1.585(3)$ & $\mathrm{C}(20)-\mathrm{F}(4)$ & $1.339(2)$ \\
\hline $\mathrm{B}(1)-\mathrm{C}(22)$ & $1.627(3)$ & $C(20)-F(5)$ & $1.340(2)$ \\
\hline $\mathrm{B}(2)-\mathrm{C}(43)$ & $1.471(3)$ & $C(20)-F(6)$ & $1.344(2)$ \\
\hline $\mathrm{B}(2)-\mathrm{N}(2)$ & $1.582(3)$ & $\mathrm{C}(21)-\mathrm{F}(8)$ & $1.318(3)$ \\
\hline $\mathrm{B}(2)-\mathrm{C}(52)$ & $1.623(3)$ & $\mathrm{C}(21)-\mathrm{F}(9)$ & $1.324(3)$ \\
\hline $\mathrm{N}(1)-\mathrm{C}(12)$ & $1.358(2)$ & $\mathrm{C}(21)-\mathrm{F}(7)$ & $1.350(3)$ \\
\hline $\mathrm{N}(1)-\mathrm{C}(8)$ & $1.372(2)$ & $C(22)-C(23)$ & $1.415(3)$ \\
\hline $\mathrm{N}(2)-\mathrm{C}(42)$ & $1.364(2)$ & $\mathrm{C}(22)-\mathrm{C}(27)$ & $1.416(2)$ \\
\hline $\mathrm{N}(2)-\mathrm{C}(38)$ & $1.370(2)$ & $C(23)-C(24)$ & $1.382(3)$ \\
\hline $\mathrm{C}(1)-\mathrm{C}(2)$ & $1.498(3)$ & $C(23)-C(29)$ & $1.500(3)$ \\
\hline$C(1)-C(14)$ & $1.567(3)$ & $C(24)-C(25)$ & $1.373(3)$ \\
\hline$C(2)-C(3)$ & $1.390(3)$ & $C(25)-C(26)$ & $1.373(3)$ \\
\hline$C(2)-C(7)$ & $1.397(3)$ & $\mathrm{C}(25)-\mathrm{C}(30)$ & $1.490(3)$ \\
\hline$C(3)-C(4)$ & $1.397(3)$ & $C(26)-C(27)$ & $1.387(3)$ \\
\hline$C(3)-C(8)$ & $1.477(3)$ & $C(27)-C(28)$ & $1.510(3)$ \\
\hline$C(4)-C(5)$ & $1.374(3)$ & $\mathrm{C}(28)-\mathrm{F}(14)$ & $1.325(2)$ \\
\hline$C(5)-C(6)$ & $1.377(3)$ & $\mathrm{C}(28)-\mathrm{F}(15)$ & $1.339(2)$ \\
\hline$C(6)-C(7)$ & $1.379(3)$ & $\mathrm{C}(28)-\mathrm{F}(13)$ & $1.340(2)$ \\
\hline $\mathrm{C}(8)-\mathrm{C}(9)$ & $1.389(3)$ & $C(29)-F(12)$ & $1.325(2)$ \\
\hline$C(9)-C(10)$ & $1.373(3)$ & $\mathrm{C}(29)-\mathrm{F}(10)$ & $1.339(2)$ \\
\hline $\mathrm{C}(10)-\mathrm{C}(11)$ & $1.380(3)$ & $\mathrm{C}(29)-\mathrm{F}(11)$ & $1.341(2)$ \\
\hline $\mathrm{C}(11)-\mathrm{C}(12)$ & $1.359(3)$ & $\mathrm{C}(30)-\mathrm{F}(18)$ & $1.248(8)$ \\
\hline $\mathrm{C}(13)-\mathrm{C}(18)$ & $1.461(3)$ & $C(30)-F(20)$ & $1.287(6)$ \\
\hline $\mathrm{C}(13)-\mathrm{C}(14)$ & $1.564(3)$ & $\mathrm{C}(30)-\mathrm{F}(21)$ & $1.289(8)$ \\
\hline$C(14)-C(15)$ & $1.509(3)$ & $\mathrm{C}(30)-\mathrm{F}(17)$ & $1.303(5)$ \\
\hline $\mathrm{C}(14)-\mathrm{C}(19)$ & $1.528(3)$ & $\mathrm{C}(30)-\mathrm{F}(19)$ & $1.385(7)$ \\
\hline$C(15)-C(16)$ & $1.322(3)$ & $\mathrm{C}(30)-\mathrm{F}(16)$ & $1.413(6)$ \\
\hline$C(16)-C(17)$ & $1.427(3)$ & $\mathrm{C}(31)-\mathrm{C}(32)$ & $1.493(3)$ \\
\hline$C(16)-C(21)$ & $1.483(3)$ & $\mathrm{C}(31)-\mathrm{C}(44)$ & $1.552(3)$ \\
\hline$C(17)-C(18)$ & $1.352(3)$ & $\mathrm{C}(32)-\mathrm{C}(37)$ & $1.391(3)$ \\
\hline$C(18)-C(20)$ & $1.509(3)$ & $C(32)-C(33)$ & $1.392(3)$ \\
\hline$C(19)-F(2)$ & $1.335(3)$ & $\mathrm{C}(33)-\mathrm{C}(34)$ & $1.393(3)$ \\
\hline$C(19)-F(3)$ & $1.347(2)$ & $C(33)-C(38)$ & $1.477(3)$ \\
\hline
\end{tabular}




\begin{tabular}{|c|c|c|c|}
\hline$C(34)-C(35)$ & $1.376(3)$ & $\mathrm{C}(58)-\mathrm{F}(38)$ & $1.347(2)$ \\
\hline$C(35)-C(36)$ & $1.381(3)$ & $C(59)-F(35 A)$ & $1.286(7)$ \\
\hline$C(36)-C(37)$ & $1.377(3)$ & $C(59)-F(36)$ & $1.310(8)$ \\
\hline $\mathrm{C}(38)-\mathrm{C}(39)$ & $1.391(3)$ & $\mathrm{C}(59)-\mathrm{F}(34)$ & $1.313(8)$ \\
\hline$C(39)-C(40)$ & $1.368(3)$ & $\mathrm{C}(59)-\mathrm{F}(36 \mathrm{~A})$ & $1.339(8)$ \\
\hline$C(40)-C(41)$ & $1.379(3)$ & $\mathrm{C}(59)-\mathrm{F}(34 \mathrm{~A})$ & $1.340(8)$ \\
\hline$C(41)-C(42)$ & $1.363(3)$ & $\mathrm{C}(59)-\mathrm{F}(35)$ & $1.351(8)$ \\
\hline$C(43)-C(48)$ & $1.458(3)$ & $\mathrm{C}(60)-\mathrm{F}(31)$ & $1.334(3)$ \\
\hline$C(43)-C(44)$ & $1.570(3)$ & $C(60)-F(32)$ & $1.340(3)$ \\
\hline$C(44)-C(45)$ & $1.506(3)$ & $\mathrm{C}(60)-\mathrm{F}(33)$ & $1.341(2)$ \\
\hline$C(44)-C(50)$ & $1.533(3)$ & & \\
\hline$C(45)-C(46)$ & $1.319(3)$ & $\mathrm{C}(13)-\mathrm{B}(1)-\mathrm{N}(1)$ & $123.71(17)$ \\
\hline$C(46)-C(47)$ & $1.428(3)$ & $\mathrm{C}(13)-\mathrm{B}(1)-\mathrm{C}(22)$ & $129.73(18)$ \\
\hline$C(46)-C(51)$ & $1.491(3)$ & $\mathrm{N}(1)-\mathrm{B}(1)-\mathrm{C}(22)$ & $106.32(16)$ \\
\hline $\mathrm{C}(47)-\mathrm{C}(48)$ & $1.355(3)$ & $\mathrm{C}(43)-\mathrm{B}(2)-\mathrm{N}(2)$ & $124.29(18)$ \\
\hline$C(48)-C(49)$ & $1.509(3)$ & $\mathrm{C}(43)-\mathrm{B}(2)-\mathrm{C}(52)$ & $129.03(18)$ \\
\hline$C(49)-F(29)$ & $1.343(2)$ & $\mathrm{N}(2)-\mathrm{B}(2)-\mathrm{C}(52)$ & $106.56(16)$ \\
\hline$C(49)-F(28)$ & $1.345(2)$ & $\mathrm{C}(12)-\mathrm{N}(1)-\mathrm{C}(8)$ & $118.21(16)$ \\
\hline$C(49)-F(30)$ & $1.346(2)$ & $\mathrm{C}(12)-\mathrm{N}(1)-\mathrm{B}(1)$ & $116.24(15)$ \\
\hline$C(50)-F(24)$ & $1.336(2)$ & $\mathrm{C}(8)-\mathrm{N}(1)-\mathrm{B}(1)$ & $124.62(16)$ \\
\hline$C(50)-F(23)$ & $1.343(2)$ & $\mathrm{C}(42)-\mathrm{N}(2)-\mathrm{C}(38)$ & $118.27(17)$ \\
\hline$C(50)-F(22)$ & $1.351(2)$ & $\mathrm{C}(42)-\mathrm{N}(2)-\mathrm{B}(2)$ & $116.36(15)$ \\
\hline$C(51)-F(27)$ & $1.328(3)$ & $\mathrm{C}(38)-\mathrm{N}(2)-\mathrm{B}(2)$ & $124.34(16)$ \\
\hline$C(51)-F(25)$ & $1.335(3)$ & $C(2)-C(1)-C(14)$ & $113.00(17)$ \\
\hline$C(51)-F(26)$ & $1.347(3)$ & $C(3)-C(2)-C(7)$ & $118.5(2)$ \\
\hline$C(52)-C(53)$ & $1.413(3)$ & $C(3)-C(2)-C(1)$ & $119.7(2)$ \\
\hline$C(52)-C(57)$ & $1.425(3)$ & $C(7)-C(2)-C(1)$ & $121.8(2)$ \\
\hline$C(53)-C(54)$ & $1.391(3)$ & $C(2)-C(3)-C(4)$ & $120.1(2)$ \\
\hline$C(53)-C(58)$ & $1.495(3)$ & $\mathrm{C}(2)-\mathrm{C}(3)-\mathrm{C}(8)$ & $120.02(18)$ \\
\hline$C(54)-C(55)$ & $1.377(4)$ & $\mathrm{C}(4)-\mathrm{C}(3)-\mathrm{C}(8)$ & $119.66(19)$ \\
\hline$C(55)-C(56)$ & $1.372(4)$ & $C(5)-C(4)-C(3)$ & $120.4(2)$ \\
\hline$C(55)-C(59)$ & $1.505(4)$ & $C(4)-C(5)-C(6)$ & $119.7(2)$ \\
\hline$C(56)-C(57)$ & $1.385(3)$ & $C(5)-C(6)-C(7)$ & $120.5(2)$ \\
\hline$C(57)-C(60)$ & $1.501(3)$ & $C(6)-C(7)-C(2)$ & $120.7(2)$ \\
\hline $\mathrm{C}(58)-\mathrm{F}(37)$ & $1.321(3)$ & $\mathrm{N}(1)-\mathrm{C}(8)-\mathrm{C}(9)$ & 119.17(19) \\
\hline C(58)-F(39) & $1.336(2)$ & $\mathrm{N}(1)-\mathrm{C}(8)-\mathrm{C}(3)$ & $121.41(17)$ \\
\hline
\end{tabular}




\begin{tabular}{|c|c|}
\hline $\mathrm{C}(9)-\mathrm{C}(8)-\mathrm{C}(3)$ & $119.42(17)$ \\
\hline $\mathrm{C}(10)-\mathrm{C}(9)-\mathrm{C}(8)$ & $121.5(2)$ \\
\hline $\mathrm{C}(9)-\mathrm{C}(10)-\mathrm{C}(11)$ & $118.6(2)$ \\
\hline $\mathrm{C}(12)-\mathrm{C}(11)-\mathrm{C}(10)$ & $118.8(2)$ \\
\hline $\mathrm{N}(1)-\mathrm{C}(12)-\mathrm{C}(11)$ & $123.58(19)$ \\
\hline $\mathrm{C}(18)-\mathrm{C}(13)-\mathrm{B}(1)$ & $123.90(17)$ \\
\hline$C(18)-C(13)-C(14)$ & $114.76(17)$ \\
\hline $\mathrm{B}(1)-\mathrm{C}(13)-\mathrm{C}(14)$ & $121.28(17)$ \\
\hline$C(15)-C(14)-C(19)$ & $105.30(18)$ \\
\hline$C(15)-C(14)-C(13)$ & $114.13(18)$ \\
\hline$C(19)-C(14)-C(13)$ & $110.55(16)$ \\
\hline $\mathrm{C}(15)-\mathrm{C}(14)-\mathrm{C}(1)$ & 106.91(17) \\
\hline$C(19)-C(14)-C(1)$ & $105.23(18)$ \\
\hline$C(13)-C(14)-C(1)$ & $113.99(15)$ \\
\hline $\mathrm{C}(16)-\mathrm{C}(15)-\mathrm{C}(14)$ & $124.0(2)$ \\
\hline$C(15)-C(16)-C(17)$ & $121.1(2)$ \\
\hline $\mathrm{C}(15)-\mathrm{C}(16)-\mathrm{C}(21)$ & $121.3(2)$ \\
\hline$C(17)-C(16)-C(21)$ & $117.4(2)$ \\
\hline $\mathrm{C}(18)-\mathrm{C}(17)-\mathrm{C}(16)$ & $120.7(2)$ \\
\hline $\mathrm{C}(17)-\mathrm{C}(18)-\mathrm{C}(13)$ & $124.77(19)$ \\
\hline $\mathrm{C}(17)-\mathrm{C}(18)-\mathrm{C}(20)$ & $113.32(19)$ \\
\hline $\mathrm{C}(13)-\mathrm{C}(18)-\mathrm{C}(20)$ & $121.36(18)$ \\
\hline $\mathrm{F}(2)-\mathrm{C}(19)-\mathrm{F}(3)$ & $106.74(19)$ \\
\hline $\mathrm{F}(2)-\mathrm{C}(19)-\mathrm{F}(1)$ & $105.69(19)$ \\
\hline $\mathrm{F}(3)-\mathrm{C}(19)-\mathrm{F}(1)$ & $105.95(16)$ \\
\hline $\mathrm{F}(2)-\mathrm{C}(19)-\mathrm{C}(14)$ & $114.13(17)$ \\
\hline $\mathrm{F}(3)-\mathrm{C}(19)-\mathrm{C}(14)$ & $112.5(2)$ \\
\hline $\mathrm{F}(1)-\mathrm{C}(19)-\mathrm{C}(14)$ & 111.31(19) \\
\hline $\mathrm{F}(4)-\mathrm{C}(20)-\mathrm{F}(5)$ & $106.09(17)$ \\
\hline $\mathrm{F}(4)-\mathrm{C}(20)-\mathrm{F}(6)$ & $104.53(16)$ \\
\hline $\mathrm{F}(5)-\mathrm{C}(20)-\mathrm{F}(6)$ & $104.60(16)$ \\
\hline $\mathrm{F}(4)-\mathrm{C}(20)-\mathrm{C}(18)$ & $112.86(16)$ \\
\hline $\mathrm{F}(5)-\mathrm{C}(20)-\mathrm{C}(18)$ & $115.08(17)$ \\
\hline $\mathrm{F}(6)-\mathrm{C}(20)-\mathrm{C}(18)$ & $112.74(18)$ \\
\hline $\mathrm{F}(8)-\mathrm{C}(21)-\mathrm{F}(9)$ & 107.2(3) \\
\hline $\mathrm{F}(8)-\mathrm{C}(21)-\mathrm{F}(7)$ & $106.4(3)$ \\
\hline
\end{tabular}

\begin{tabular}{ll}
$F(9)-C(21)-F(7)$ & $103.7(2)$ \\
$F(8)-C(21)-C(16)$ & $112.8(2)$ \\
$F(9)-C(21)-C(16)$ & $113.1(2)$ \\
$F(7)-C(21)-C(16)$ & $113.0(3)$ \\
$C(23)-C(22)-C(27)$ & $114.37(17)$ \\
$C(23)-C(22)-B(1)$ & $122.66(16)$ \\
$C(27)-C(22)-B(1)$ & $122.70(16)$ \\
$C(24)-C(23)-C(22)$ & $122.71(18)$ \\
$C(24)-C(23)-C(29)$ & $114.07(17)$ \\
$C(22)-C(23)-C(29)$ & $123.18(17)$ \\
$C(25)-C(24)-C(23)$ & $120.6(2)$ \\
$C(24)-C(25)-C(26)$ & $119.2(2)$ \\
$C(24)-C(25)-C(30)$ & $119.6(2)$ \\
$C(26)-C(25)-C(30)$ & $121.2(2)$ \\
$C(25)-C(26)-C(27)$ & $120.60(19)$ \\
$C(26)-C(27)-C(22)$ & $122.48(18)$ \\
$C(26)-C(27)-C(28)$ & $115.21(17)$ \\
$C(22)-C(27)-C(28)$ & $122.29(17)$ \\
$F(14)-C(28)-F(15)$ & $106.61(17)$ \\
$F(14)-C(28)-F(13)$ & $105.61(16)$ \\
$F(15)-C(28)-F(13)$ & $105.04(16)$ \\
$F(14)-C(28)-C(27)$ & $115.06(17)$ \\
$F(15)-C(28)-C(27)$ & $111.72(17)$ \\
$F(13)-C(28)-C(27)$ & $112.08(17)$ \\
$F(12)-C(29)-F(10)$ & $106.34(17)$ \\
$F(12)-C(29)-F(11)$ & $106.11(17)$ \\
$F(10)-C(29)-F(11)$ & $106.11(17)$ \\
$F(12)-C(29)-C(23)$ & $114.49(17)$ \\
$F(10)-C(29)-C(23)$ & $111.60(18)$ \\
$F(11)-C(29)-C(23)$ & $111.64(17)$ \\
$F(18)-C(30)-F(20)$ & $103.9(7)$ \\
$F(18)-C(30)-F(21)$ & $118.6(8)$ \\
$F(20)-C(30)-F(17)$ & $128.3(5)$ \\
$F(21)-C(30)-F(17)$ & $117.6(7)$ \\
& $105.9(7)$ \\
\hline &
\end{tabular}




\begin{tabular}{|c|c|}
\hline$F(20)-C(30)-F(16)$ & $100.1(6)$ \\
\hline $\mathrm{F}(18)-\mathrm{C}(30)-\mathrm{C}(25)$ & $116.2(5)$ \\
\hline $\mathrm{F}(20)-\mathrm{C}(30)-\mathrm{C}(25)$ & $114.0(5)$ \\
\hline $\mathrm{F}(21)-\mathrm{C}(30)-\mathrm{C}(25)$ & $114.6(6)$ \\
\hline $\mathrm{F}(17)-\mathrm{C}(30)-\mathrm{C}(25)$ & $115.6(3)$ \\
\hline $\mathrm{F}(19)-\mathrm{C}(30)-\mathrm{C}(25)$ & 107.1(4) \\
\hline $\mathrm{F}(16)-\mathrm{C}(30)-\mathrm{C}(25)$ & 107.3(3) \\
\hline $\mathrm{C}(32)-\mathrm{C}(31)-\mathrm{C}(44)$ & $113.15(16)$ \\
\hline $\mathrm{C}(37)-\mathrm{C}(32)-\mathrm{C}(33)$ & $118.7(2)$ \\
\hline $\mathrm{C}(37)-\mathrm{C}(32)-\mathrm{C}(31)$ & $121.46(19)$ \\
\hline $\mathrm{C}(33)-\mathrm{C}(32)-\mathrm{C}(31)$ & $119.80(19)$ \\
\hline $\mathrm{C}(32)-\mathrm{C}(33)-\mathrm{C}(34)$ & $119.9(2)$ \\
\hline $\mathrm{C}(32)-\mathrm{C}(33)-\mathrm{C}(38)$ & $120.25(18)$ \\
\hline $\mathrm{C}(34)-\mathrm{C}(33)-\mathrm{C}(38)$ & $119.56(19)$ \\
\hline $\mathrm{C}(35)-\mathrm{C}(34)-\mathrm{C}(33)$ & $120.3(2)$ \\
\hline $\mathrm{C}(34)-\mathrm{C}(35)-\mathrm{C}(36)$ & $120.1(2)$ \\
\hline $\mathrm{C}(37)-\mathrm{C}(36)-\mathrm{C}(35)$ & $119.8(2)$ \\
\hline $\mathrm{C}(36)-\mathrm{C}(37)-\mathrm{C}(32)$ & $121.1(2)$ \\
\hline $\mathrm{N}(2)-\mathrm{C}(38)-\mathrm{C}(39)$ & $119.15(19)$ \\
\hline $\mathrm{N}(2)-\mathrm{C}(38)-\mathrm{C}(33)$ & $122.24(17)$ \\
\hline $\mathrm{C}(39)-\mathrm{C}(38)-\mathrm{C}(33)$ & $118.60(18)$ \\
\hline $\mathrm{C}(40)-\mathrm{C}(39)-\mathrm{C}(38)$ & $121.6(2)$ \\
\hline $\mathrm{C}(39)-\mathrm{C}(40)-\mathrm{C}(41)$ & $118.9(2)$ \\
\hline$C(42)-C(41)-C(40)$ & $118.7(2)$ \\
\hline $\mathrm{C}(41)-\mathrm{C}(42)-\mathrm{N}(2)$ & $123.31(19)$ \\
\hline $\mathrm{C}(48)-\mathrm{C}(43)-\mathrm{B}(2)$ & $123.69(18)$ \\
\hline$C(48)-C(43)-C(44)$ & $114.73(17)$ \\
\hline $\mathrm{B}(2)-\mathrm{C}(43)-\mathrm{C}(44)$ & $121.53(17)$ \\
\hline$C(45)-C(44)-C(50)$ & $104.06(16)$ \\
\hline$C(45)-C(44)-C(31)$ & $107.57(16)$ \\
\hline $\mathrm{C}(50)-\mathrm{C}(44)-\mathrm{C}(31)$ & $105.51(17)$ \\
\hline$C(45)-C(44)-C(43)$ & $114.31(17)$ \\
\hline $\mathrm{C}(50)-\mathrm{C}(44)-\mathrm{C}(43)$ & $110.04(16)$ \\
\hline $\mathrm{C}(31)-\mathrm{C}(44)-\mathrm{C}(43)$ & $114.50(15)$ \\
\hline $\mathrm{C}(46)-\mathrm{C}(45)-\mathrm{C}(44)$ & $123.7(2)$ \\
\hline $\mathrm{C}(45)-\mathrm{C}(46)-\mathrm{C}(47)$ & $121.3(2)$ \\
\hline
\end{tabular}

\begin{tabular}{|c|c|}
\hline$C(45)-C(46)-C(51)$ & $121.6(2)$ \\
\hline$C(47)-C(46)-C(51)$ & $117.0(2)$ \\
\hline $\mathrm{C}(48)-\mathrm{C}(47)-\mathrm{C}(46)$ & $120.8(2)$ \\
\hline $\mathrm{C}(47)-\mathrm{C}(48)-\mathrm{C}(43)$ & $124.51(19)$ \\
\hline $\mathrm{C}(47)-\mathrm{C}(48)-\mathrm{C}(49)$ & 113.11(19) \\
\hline $\mathrm{C}(43)-\mathrm{C}(48)-\mathrm{C}(49)$ & $121.94(18)$ \\
\hline $\mathrm{F}(29)-\mathrm{C}(49)-\mathrm{F}(28)$ & $104.47(16)$ \\
\hline $\mathrm{F}(29)-\mathrm{C}(49)-\mathrm{F}(30)$ & $105.80(17)$ \\
\hline $\mathrm{F}(28)-\mathrm{C}(49)-\mathrm{F}(30)$ & $104.18(16)$ \\
\hline $\mathrm{F}(29)-\mathrm{C}(49)-\mathrm{C}(48)$ & $113.60(16)$ \\
\hline $\mathrm{F}(28)-\mathrm{C}(49)-\mathrm{C}(48)$ & $112.86(18)$ \\
\hline $\mathrm{F}(30)-\mathrm{C}(49)-\mathrm{C}(48)$ & 114.91(16) \\
\hline $\mathrm{F}(24)-\mathrm{C}(50)-\mathrm{F}(23)$ & $107.07(18)$ \\
\hline $\mathrm{F}(24)-\mathrm{C}(50)-\mathrm{F}(22)$ & $106.24(17)$ \\
\hline $\mathrm{F}(23)-\mathrm{C}(50)-\mathrm{F}(22)$ & $105.38(18)$ \\
\hline $\mathrm{F}(24)-\mathrm{C}(50)-\mathrm{C}(44)$ & 113.11(18) \\
\hline $\mathrm{F}(23)-\mathrm{C}(50)-\mathrm{C}(44)$ & $113.18(17)$ \\
\hline $\mathrm{F}(22)-\mathrm{C}(50)-\mathrm{C}(44)$ & $111.32(18)$ \\
\hline $\mathrm{F}(27)-\mathrm{C}(51)-\mathrm{F}(25)$ & $106.26(19)$ \\
\hline $\mathrm{F}(27)-\mathrm{C}(51)-\mathrm{F}(26)$ & $105.0(2)$ \\
\hline $\mathrm{F}(25)-\mathrm{C}(51)-\mathrm{F}(26)$ & $106.3(2)$ \\
\hline $\mathrm{F}(27)-\mathrm{C}(51)-\mathrm{C}(46)$ & $112.39(19)$ \\
\hline $\mathrm{F}(25)-\mathrm{C}(51)-\mathrm{C}(46)$ & $114.1(2)$ \\
\hline $\mathrm{F}(26)-\mathrm{C}(51)-\mathrm{C}(46)$ & $112.10(19)$ \\
\hline$C(53)-C(52)-C(57)$ & $113.98(19)$ \\
\hline $\mathrm{C}(53)-\mathrm{C}(52)-\mathrm{B}(2)$ & $123.33(17)$ \\
\hline $\mathrm{C}(57)-\mathrm{C}(52)-\mathrm{B}(2)$ & $122.59(18)$ \\
\hline$C(54)-C(53)-C(52)$ & $123.6(2)$ \\
\hline $\mathrm{C}(54)-\mathrm{C}(53)-\mathrm{C}(58)$ & $113.9(2)$ \\
\hline $\mathrm{C}(52)-\mathrm{C}(53)-\mathrm{C}(58)$ & $122.42(19)$ \\
\hline$C(55)-C(54)-C(53)$ & $119.4(2)$ \\
\hline$C(56)-C(55)-C(54)$ & $119.9(2)$ \\
\hline$C(56)-C(55)-C(59)$ & $120.0(3)$ \\
\hline$C(54)-C(55)-C(59)$ & $120.1(3)$ \\
\hline $\mathrm{C}(55)-\mathrm{C}(56)-\mathrm{C}(57)$ & $120.7(2)$ \\
\hline$C(56)-C(57)-C(52)$ & $122.4(2)$ \\
\hline
\end{tabular}




$\begin{array}{llll}\mathrm{C}(56)-\mathrm{C}(57)-\mathrm{C}(60) & 115.9(2) & \mathrm{F}(36)-\mathrm{C}(59)-\mathrm{F}(35) & 109.0(10) \\ \mathrm{C}(52)-\mathrm{C}(57)-\mathrm{C}(60) & 121.72(19) & \mathrm{F}(34 \mathrm{~A})-\mathrm{C}(59)-\mathrm{F}(35) & 129.2(8) \\ \mathrm{F}(37)-\mathrm{C}(58)-\mathrm{F}(39) & 106.19(19) & \mathrm{F}(35 \mathrm{~A})-\mathrm{C}(59)-\mathrm{C}(55) & 116.5(6) \\ \mathrm{F}(37)-\mathrm{C}(58)-\mathrm{F}(38) & 105.2(2) & \mathrm{F}(36)-\mathrm{C}(59)-\mathrm{C}(55) & 113.1(8) \\ \mathrm{F}(39)-\mathrm{C}(58)-\mathrm{F}(38) & 106.19(17) & \mathrm{F}(34)-\mathrm{C}(59)-\mathrm{C}(55) & 109.6(5) \\ \mathrm{F}(37)-\mathrm{C}(58)-\mathrm{C}(53) & 115.18(18) & \mathrm{F}(36 \mathrm{~A})-\mathrm{C}(59)-\mathrm{C}(55) & 109.9(6) \\ \mathrm{F}(39)-\mathrm{C}(58)-\mathrm{C}(53) & 111.9(2) & \mathrm{F}(34 \mathrm{~A})-\mathrm{C}(59)-\mathrm{C}(55) & 112.4(6) \\ \mathrm{F}(38)-\mathrm{C}(58)-\mathrm{C}(53) & 111.52(19) & \mathrm{F}(35)-\mathrm{C}(59)-\mathrm{C}(55) & 112.4(6) \\ \mathrm{F}(35 \mathrm{~A})-\mathrm{C}(59)-\mathrm{F}(36) & 111.1(9) & \mathrm{F}(31)-\mathrm{C}(60)-\mathrm{F}(32) & 106.1(2) \\ \mathrm{F}(36)-\mathrm{C}(59)-\mathrm{F}(34) & 123.3(8) & \mathrm{F}(31)-\mathrm{C}(60)-\mathrm{F}(33) & 105.23(18) \\ \mathrm{F}(35 \mathrm{~A})-\mathrm{C}(59)-\mathrm{F}(36 \mathrm{~A}) & 119.0(8) & \mathrm{F}(32)-\mathrm{C}(60)-\mathrm{F}(33) & 105.4(2) \\ \mathrm{F}(34)-\mathrm{C}(59)-\mathrm{F}(36 \mathrm{~A}) & 115.7(7) & \mathrm{F}(31)-\mathrm{C}(60)-\mathrm{C}(57) & 112.76(19) \\ \mathrm{F}(34)-\mathrm{C}(59)-\mathrm{F}(34 \mathrm{~A}) & 100.1(7) & \mathrm{F}(32)-\mathrm{C}(60)-\mathrm{C}(57) & 114.37(19) \\ \mathrm{F}(36 \mathrm{~A})-\mathrm{C}(59)-\mathrm{F}(34 \mathrm{~A}) & 108.9(9) & \mathrm{F}(33)-\mathrm{C}(60)-\mathrm{C}(57) & 112.3(2)\end{array}$


Table 7.1J. Crystal data and structure refinement for BN-4b.

Identification code

Empirical formula

Formula weight

Temperature

Wavelength

Crystal system

Space group

Unit cell dimensions

Volume

Z

Density (calculated)

Absorption coefficient

$\mathrm{F}(000)$

Crystal size

Theta range for data collection

Index ranges

Reflections collected

Independent reflections

Completeness to theta $=27.27^{\circ}$

Absorption correction

Max. and min. transmission

Refinement method

Data / restraints / parameters

Goodness-of-fit on $\mathrm{F}^{2}$

Final $\mathrm{R}$ indices [I $>2 \operatorname{sigma}(\mathrm{I})]$

$\mathrm{R}$ indices (all data)

Largest diff. peak and hole dy 38

C24 H10 B F10 N

513.14

296(2) K

$0.71073 \AA$

Monoclinic

$\mathrm{P} 2(1) / \mathrm{n}$

$\mathrm{a}=9.7376(3) \AA$

$\alpha=90^{\circ}$.

$\mathrm{b}=16.5554(5) \AA$

$\beta=101.085(2)^{\circ}$.

$\mathrm{c}=12.6822(4) \AA$

$\gamma=90^{\circ}$.
4

$1.699 \mathrm{Mg} / \mathrm{m}^{3}$

$0.164 \mathrm{~mm}^{-1}$

1024

$0.20 \times 0.08 \times 0.08 \mathrm{~mm}^{3}$

2.05 to $27.27^{\circ}$.

$-12<=\mathrm{h}<=11,-21<=\mathrm{k}<=18,-16<=\mathrm{l}<=7$

11194

$4458[\mathrm{R}(\mathrm{int})=0.0220]$

$99.0 \%$

Semi-empirical from equivalents

0.9870 and 0.9679

Full-matrix least-squares on $\mathrm{F}^{2}$

4458 / 0 / 325

1.031

$\mathrm{R} 1=0.0367, \mathrm{wR} 2=0.0898$

$\mathrm{R} 1=0.0481, \mathrm{wR} 2=0.0980$

0.264 and -0.248 e. $\AA^{-3}$ 
Table 7.1K. Bond lengths $[\AA]$ and angles $\left[^{\circ}\right]$ for BN-4b.

\begin{tabular}{|c|c|c|c|}
\hline $\mathrm{B}(1)-\mathrm{F}(1)$ & $1.4084(18)$ & $C(19)-C(24)$ & $1.384(2)$ \\
\hline $\mathrm{B}(1)-\mathrm{C}(13)$ & $1.628(2)$ & $C(19)-C(20)$ & $1.389(2)$ \\
\hline $\mathrm{B}(1)-\mathrm{C}(19)$ & $1.636(2)$ & $\mathrm{C}(20)-\mathrm{C}(21)$ & $1.380(2)$ \\
\hline $\mathrm{B}(1)-\mathrm{N}(1)$ & $1.658(2)$ & $\mathrm{C}(21)-\mathrm{C}(22)$ & $1.372(2)$ \\
\hline $\mathrm{N}(1)-\mathrm{C}(12)$ & $1.3501(19)$ & $C(22)-C(23)$ & $1.370(2)$ \\
\hline $\mathrm{N}(1)-\mathrm{C}(8)$ & $1.3564(19)$ & $C(23)-C(24)$ & $1.384(2)$ \\
\hline $\mathrm{F}(2)-\mathrm{C}(15)$ & $1.3529(17)$ & & \\
\hline $\mathrm{F}(3)-\mathrm{C}(16)$ & $1.3441(17)$ & $\mathrm{F}(1)-\mathrm{B}(1)-\mathrm{C}(13)$ & $108.45(11)$ \\
\hline $\mathrm{F}(4)-\mathrm{C}(17)$ & $1.3455(18)$ & $\mathrm{F}(1)-\mathrm{B}(1)-\mathrm{C}(19)$ & $108.80(11)$ \\
\hline $\mathrm{F}(5)-\mathrm{C}(18)$ & $1.3511(17)$ & $\mathrm{C}(13)-\mathrm{B}(1)-\mathrm{C}(19)$ & $117.58(12)$ \\
\hline $\mathrm{F}(6)-\mathrm{C}(24)$ & $1.3433(16)$ & $\mathrm{F}(1)-\mathrm{B}(1)-\mathrm{N}(1)$ & $102.68(11)$ \\
\hline $\mathrm{F}(7)-\mathrm{C}(23)$ & $1.3464(18)$ & $\mathrm{C}(13)-\mathrm{B}(1)-\mathrm{N}(1)$ & $112.88(11)$ \\
\hline $\mathrm{F}(8)-\mathrm{C}(22)$ & $1.3408(17)$ & $\mathrm{C}(19)-\mathrm{B}(1)-\mathrm{N}(1)$ & $105.39(11)$ \\
\hline $\mathrm{F}(9)-\mathrm{C}(21)$ & $1.3381(17)$ & $\mathrm{C}(12)-\mathrm{N}(1)-\mathrm{C}(8)$ & 118.91(13) \\
\hline $\mathrm{F}(10)-\mathrm{C}(20)$ & $1.3524(17)$ & $\mathrm{C}(12)-\mathrm{N}(1)-\mathrm{B}(1)$ & $117.29(12)$ \\
\hline $\mathrm{C}(1)-\mathrm{C}(2)$ & $1.510(2)$ & $\mathrm{C}(8)-\mathrm{N}(1)-\mathrm{B}(1)$ & $122.82(11)$ \\
\hline$C(1)-C(14)$ & $1.514(2)$ & $\mathrm{C}(2)-\mathrm{C}(1)-\mathrm{C}(14)$ & $111.00(12)$ \\
\hline$C(2)-C(3)$ & $1.393(2)$ & $\mathrm{C}(3)-\mathrm{C}(2)-\mathrm{C}(7)$ & $118.52(13)$ \\
\hline$C(2)-C(7)$ & $1.397(2)$ & $\mathrm{C}(3)-\mathrm{C}(2)-\mathrm{C}(1)$ & $120.03(14)$ \\
\hline$C(3)-C(4)$ & $1.382(2)$ & $\mathrm{C}(7)-\mathrm{C}(2)-\mathrm{C}(1)$ & $121.45(13)$ \\
\hline$C(4)-C(5)$ & $1.382(3)$ & $\mathrm{C}(4)-\mathrm{C}(3)-\mathrm{C}(2)$ & $120.74(16)$ \\
\hline$C(5)-C(6)$ & $1.377(2)$ & $\mathrm{C}(3)-\mathrm{C}(4)-\mathrm{C}(5)$ & $120.40(16)$ \\
\hline$C(6)-C(7)$ & $1.394(2)$ & $C(6)-C(5)-C(4)$ & $119.72(15)$ \\
\hline$C(7)-C(8)$ & $1.480(2)$ & $C(5)-C(6)-C(7)$ & $120.35(16)$ \\
\hline $\mathrm{C}(8)-\mathrm{C}(9)$ & $1.386(2)$ & $C(6)-C(7)-C(2)$ & $120.22(14)$ \\
\hline$C(9)-C(10)$ & $1.377(2)$ & $C(6)-C(7)-C(8)$ & $120.72(14)$ \\
\hline$C(10)-C(11)$ & $1.377(3)$ & $C(2)-C(7)-C(8)$ & $118.96(13)$ \\
\hline$C(11)-C(12)$ & $1.375(2)$ & $\mathrm{N}(1)-\mathrm{C}(8)-\mathrm{C}(9)$ & $120.23(14)$ \\
\hline$C(13)-C(18)$ & $1.386(2)$ & $\mathrm{N}(1)-\mathrm{C}(8)-\mathrm{C}(7)$ & $119.01(12)$ \\
\hline$C(13)-C(14)$ & $1.408(2)$ & $\mathrm{C}(9)-\mathrm{C}(8)-\mathrm{C}(7)$ & $120.66(14)$ \\
\hline$C(14)-C(15)$ & $1.384(2)$ & $\mathrm{C}(10)-\mathrm{C}(9)-\mathrm{C}(8)$ & $120.18(16)$ \\
\hline$C(15)-C(16)$ & $1.372(2)$ & $C(9)-C(10)-C(11)$ & $119.07(16)$ \\
\hline$C(16)-C(17)$ & $1.366(2)$ & $\mathrm{C}(9)-\mathrm{C}(10)-\mathrm{H}(10 \mathrm{~A})$ & 120.5 \\
\hline$C(17)-C(18)$ & $1.377(2)$ & $\mathrm{C}(11)-\mathrm{C}(10)-\mathrm{H}(10 \mathrm{~A})$ & 120.5 \\
\hline
\end{tabular}




$\begin{array}{ll}\mathrm{C}(12)-\mathrm{C}(11)-\mathrm{C}(10) & 118.84(16) \\ \mathrm{N}(1)-\mathrm{C}(12)-\mathrm{C}(11) & 122.33(16) \\ \mathrm{C}(18)-\mathrm{C}(13)-\mathrm{C}(14) & 116.00(13) \\ \mathrm{C}(18)-\mathrm{C}(13)-\mathrm{B}(1) & 116.03(12) \\ \mathrm{C}(14)-\mathrm{C}(13)-\mathrm{B}(1) & 127.87(13) \\ \mathrm{C}(15)-\mathrm{C}(14)-\mathrm{C}(13) & 119.19(13) \\ \mathrm{C}(15)-\mathrm{C}(14)-\mathrm{C}(1) & 117.86(13) \\ \mathrm{C}(13)-\mathrm{C}(14)-\mathrm{C}(1) & 122.90(13) \\ \mathrm{F}(2)-\mathrm{C}(15)-\mathrm{C}(16) & 116.74(13) \\ \mathrm{F}(2)-\mathrm{C}(15)-\mathrm{C}(14) & 120.32(14) \\ \mathrm{C}(16)-\mathrm{C}(15)-\mathrm{C}(14) & 122.95(14) \\ \mathrm{F}(3)-\mathrm{C}(16)-\mathrm{C}(17) & 120.45(15) \\ \mathrm{F}(3)-\mathrm{C}(16)-\mathrm{C}(15) & 121.01(15) \\ \mathrm{C}(17)-\mathrm{C}(16)-\mathrm{C}(15) & 118.53(14) \\ \mathrm{F}(4)-\mathrm{C}(17)-\mathrm{C}(16) & 119.97(14) \\ \mathrm{F}(4)-\mathrm{C}(17)-\mathrm{C}(18) & 120.89(14) \\ \mathrm{C}(16)-\mathrm{C}(17)-\mathrm{C}(18) & 119.11(14) \\ \mathrm{F}(5)-\mathrm{C}(18)-\mathrm{C}(17) & 115.36(13) \\ \mathrm{F}(5)-\mathrm{C}(18)-\mathrm{C}(13) & 120.61(13) \\ \mathrm{C}(17)-\mathrm{C}(18)-\mathrm{C}(13) & 124.03(14)\end{array}$

$\begin{array}{ll}\mathrm{C}(24)-\mathrm{C}(19)-\mathrm{C}(20) & 114.19(13) \\ \mathrm{C}(24)-\mathrm{C}(19)-\mathrm{B}(1) & 126.36(13) \\ \mathrm{C}(20)-\mathrm{C}(19)-\mathrm{B}(1) & 119.42(13) \\ \mathrm{F}(10)-\mathrm{C}(20)-\mathrm{C}(21) & 116.37(13) \\ \mathrm{F}(10)-\mathrm{C}(20)-\mathrm{C}(19) & 119.39(13) \\ \mathrm{C}(21)-\mathrm{C}(20)-\mathrm{C}(19) & 124.21(14) \\ \mathrm{F}(9)-\mathrm{C}(21)-\mathrm{C}(22) & 120.01(13) \\ \mathrm{F}(9)-\mathrm{C}(21)-\mathrm{C}(20) & 120.96(14) \\ \mathrm{C}(22)-\mathrm{C}(21)-\mathrm{C}(20) & 119.02(14) \\ \mathrm{F}(8)-\mathrm{C}(22)-\mathrm{C}(23) & 120.39(15) \\ \mathrm{F}(8)-\mathrm{C}(22)-\mathrm{C}(21) & 120.26(14) \\ \mathrm{C}(23)-\mathrm{C}(22)-\mathrm{C}(21) & 119.36(13) \\ \mathrm{F}(7)-\mathrm{C}(23)-\mathrm{C}(22) & 119.76(13) \\ \mathrm{F}(7)-\mathrm{C}(23)-\mathrm{C}(24) & 120.26(14) \\ \mathrm{C}(22)-\mathrm{C}(23)-\mathrm{C}(24) & 119.98(14) \\ \mathrm{F}(6)-\mathrm{C}(24)-\mathrm{C}(19) & 120.97(12) \\ \mathrm{F}(6)-\mathrm{C}(24)-\mathrm{C}(23) & 115.82(13) \\ \mathrm{C}(19)-\mathrm{C}(24)-\mathrm{C}(23) & 123.18(13)\end{array}$


Table 7.1L. Crystal data and structure refinement for BN-5.

Identification code

Empirical formula

Formula weight

Temperature

Wavelength

Crystal system

Space group

Unit cell dimensions

Volume

Z

Density (calculated)

Absorption coefficient

$\mathrm{F}(000)$

Crystal size

Theta range for data collection

Index ranges

Reflections collected

Independent reflections

Completeness to theta $=27.30^{\circ}$

Absorption correction

Max. and min. transmission

Refinement method

Data / restraints / parameters

Goodness-of-fit on $\mathrm{F}^{2}$

Final $\mathrm{R}$ indices [I $>2 \operatorname{sigma}(\mathrm{I})]$

$\mathrm{R}$ indices (all data)

Largest diff. peak and hole dy 42

C24 H15 B F5 N

423.18

296(2) K

$0.71073 \AA$

Monoclinic

$\mathrm{P} 2(1) / \mathrm{c}$

$\mathrm{a}=13.8288(5) \AA$

$\alpha=90^{\circ}$.

$\mathrm{b}=8.7441(3) \AA$

$\beta=111.064(2)^{\circ}$.

$\mathrm{c}=17.1220(6) \AA$

$\gamma=90^{\circ}$.

1932.05(12) $\AA^{3}$

4

$1.455 \mathrm{Mg} / \mathrm{m}^{3}$

$0.118 \mathrm{~mm}^{-1}$

864

$0.10 \times 0.10 \times 0.08 \mathrm{~mm}^{3}$

1.58 to $27.30^{\circ}$.

$-17<=\mathrm{h}<=17,-11<=\mathrm{k}<=11,-21<=\mathrm{l}<=21$

16541

$4317[\mathrm{R}(\mathrm{int})=0.0332]$

$99.4 \%$

Semi-empirical from equivalents

0.9906 and 0.9883

Full-matrix least-squares on $\mathrm{F}^{2}$

4317 / 0 / 280

1.040

$\mathrm{R} 1=0.0462, \mathrm{wR} 2=0.1081$

$\mathrm{R} 1=0.0792, \mathrm{wR} 2=0.1274$

0.189 and -0.230 e. $\AA^{-3}$ 
Table 7.1M. Bond lengths $[\AA]$ and angles $\left[{ }^{\circ}\right]$ for BN-5.

\begin{tabular}{|c|c|c|c|}
\hline $\mathrm{B}(1)-\mathrm{C}(1)$ & $1.612(3)$ & $C(23)-C(24)$ & $1.382(3)$ \\
\hline B(1)-C(19) & $1.615(3)$ & & \\
\hline $\mathrm{B}(1)-\mathrm{N}(1)$ & $1.635(2)$ & $\mathrm{C}(1)-\mathrm{B}(1)-\mathrm{C}(19)$ & $113.11(14)$ \\
\hline B(1)-C(13) & $1.661(2)$ & $\mathrm{C}(1)-\mathrm{B}(1)-\mathrm{N}(1)$ & $101.89(14)$ \\
\hline $\mathrm{N}(1)-\mathrm{C}(12)$ & $1.346(2)$ & $\mathrm{C}(19)-\mathrm{B}(1)-\mathrm{N}(1)$ & 109.91(14) \\
\hline $\mathrm{N}(1)-\mathrm{C}(8)$ & $1.363(2)$ & $\mathrm{C}(1)-\mathrm{B}(1)-\mathrm{C}(13)$ & $113.61(15)$ \\
\hline $\mathrm{F}(1)-\mathrm{C}(14)$ & $1.359(2)$ & $\mathrm{C}(19)-\mathrm{B}(1)-\mathrm{C}(13)$ & $111.12(14)$ \\
\hline $\mathrm{F}(2)-\mathrm{C}(15)$ & $1.347(2)$ & $\mathrm{N}(1)-\mathrm{B}(1)-\mathrm{C}(13)$ & $106.56(13)$ \\
\hline $\mathrm{F}(3)-\mathrm{C}(16)$ & $1.342(2)$ & $\mathrm{C}(12)-\mathrm{N}(1)-\mathrm{C}(8)$ & $119.49(16)$ \\
\hline $\mathrm{F}(4)-\mathrm{C}(17)$ & $1.343(2)$ & $\mathrm{C}(12)-\mathrm{N}(1)-\mathrm{B}(1)$ & $120.35(15)$ \\
\hline $\mathrm{F}(5)-\mathrm{C}(18)$ & $1.3507(19)$ & $\mathrm{C}(8)-\mathrm{N}(1)-\mathrm{B}(1)$ & $120.16(14)$ \\
\hline $\mathrm{C}(1)-\mathrm{C}(2)$ & $1.489(2)$ & $\mathrm{C}(2)-\mathrm{C}(1)-\mathrm{B}(1)$ & $111.26(15)$ \\
\hline$C(2)-C(3)$ & $1.392(3)$ & $\mathrm{C}(3)-\mathrm{C}(2)-\mathrm{C}(7)$ & $118.48(19)$ \\
\hline$C(2)-C(7)$ & $1.402(3)$ & $\mathrm{C}(3)-\mathrm{C}(2)-\mathrm{C}(1)$ & $122.78(18)$ \\
\hline$C(3)-C(4)$ & $1.377(3)$ & $C(7)-C(2)-C(1)$ & $118.73(17)$ \\
\hline$C(4)-C(5)$ & $1.372(4)$ & $\mathrm{C}(4)-\mathrm{C}(3)-\mathrm{C}(2)$ & $121.3(2)$ \\
\hline$C(5)-C(6)$ & $1.381(3)$ & $\mathrm{C}(5)-\mathrm{C}(4)-\mathrm{C}(3)$ & $120.1(2)$ \\
\hline$C(6)-C(7)$ & $1.399(3)$ & $\mathrm{C}(4)-\mathrm{C}(5)-\mathrm{C}(6)$ & $120.1(2)$ \\
\hline$C(7)-C(8)$ & $1.480(3)$ & $\mathrm{C}(5)-\mathrm{C}(6)-\mathrm{C}(7)$ & $120.4(2)$ \\
\hline$C(8)-C(9)$ & $1.383(3)$ & $\mathrm{C}(6)-\mathrm{C}(7)-\mathrm{C}(2)$ & $119.58(19)$ \\
\hline$C(9)-C(10)$ & $1.376(3)$ & $\mathrm{C}(6)-\mathrm{C}(7)-\mathrm{C}(8)$ & $120.49(19)$ \\
\hline$C(10)-C(11)$ & $1.372(3)$ & $\mathrm{C}(2)-\mathrm{C}(7)-\mathrm{C}(8)$ & $119.88(17)$ \\
\hline$C(11)-C(12)$ & $1.371(3)$ & $\mathrm{N}(1)-\mathrm{C}(8)-\mathrm{C}(9)$ & $118.88(18)$ \\
\hline$C(13)-C(14)$ & $1.378(2)$ & $\mathrm{N}(1)-\mathrm{C}(8)-\mathrm{C}(7)$ & $119.48(16)$ \\
\hline$C(13)-C(18)$ & $1.387(2)$ & $\mathrm{C}(9)-\mathrm{C}(8)-\mathrm{C}(7)$ & $121.61(18)$ \\
\hline$C(14)-C(15)$ & $1.378(3)$ & $\mathrm{C}(10)-\mathrm{C}(9)-\mathrm{C}(8)$ & $121.1(2)$ \\
\hline$C(15)-C(16)$ & $1.366(3)$ & $\mathrm{C}(11)-\mathrm{C}(10)-\mathrm{C}(9)$ & $119.3(2)$ \\
\hline$C(16)-C(17)$ & $1.358(3)$ & $C(12)-C(11)-C(10)$ & $118.3(2)$ \\
\hline$C(17)-C(18)$ & $1.382(3)$ & $\mathrm{N}(1)-\mathrm{C}(12)-\mathrm{C}(11)$ & $122.8(2)$ \\
\hline C(19)-C(24) & $1.388(2)$ & $\mathrm{C}(14)-\mathrm{C}(13)-\mathrm{C}(18)$ & $112.36(16)$ \\
\hline$C(19)-C(20)$ & $1.391(2)$ & $\mathrm{C}(14)-\mathrm{C}(13)-\mathrm{B}(1)$ & $123.86(15)$ \\
\hline$C(20)-C(21)$ & $1.380(3)$ & $\mathrm{C}(18)-\mathrm{C}(13)-\mathrm{B}(1)$ & $123.58(15)$ \\
\hline$C(21)-C(22)$ & $1.375(3)$ & $\mathrm{F}(1)-\mathrm{C}(14)-\mathrm{C}(13)$ & $120.75(16)$ \\
\hline$C(22)-C(23)$ & $1.363(3)$ & $\mathrm{F}(1)-\mathrm{C}(14)-\mathrm{C}(15)$ & $114.75(16)$ \\
\hline
\end{tabular}




$\begin{array}{llll}\mathrm{C}(13)-\mathrm{C}(14)-\mathrm{C}(15) & 124.50(17) & \mathrm{F}(5)-\mathrm{C}(18)-\mathrm{C}(13) & 120.07(15) \\ \mathrm{F}(2)-\mathrm{C}(15)-\mathrm{C}(16) & 119.28(18) & \mathrm{C}(17)-\mathrm{C}(18)-\mathrm{C}(13) & 125.13(17) \\ \mathrm{F}(2)-\mathrm{C}(15)-\mathrm{C}(14) & 120.69(19) & \mathrm{C}(24)-\mathrm{C}(19)-\mathrm{C}(20) & 115.00(16) \\ \mathrm{C}(16)-\mathrm{C}(15)-\mathrm{C}(14) & 120.03(18) & \mathrm{C}(24)-\mathrm{C}(19)-\mathrm{B}(1) & 123.79(15) \\ \mathrm{F}(3)-\mathrm{C}(16)-\mathrm{C}(17) & 120.64(19) & \mathrm{C}(20)-\mathrm{C}(19)-\mathrm{B}(1) & 121.19(15) \\ \mathrm{F}(3)-\mathrm{C}(16)-\mathrm{C}(15) & 120.60(19) & \mathrm{C}(21)-\mathrm{C}(20)-\mathrm{C}(19) & 122.64(18) \\ \mathrm{C}(17)-\mathrm{C}(16)-\mathrm{C}(15) & 118.76(17) & \mathrm{C}(22)-\mathrm{C}(21)-\mathrm{C}(20) & 120.30(19) \\ \mathrm{F}(4)-\mathrm{C}(17)-\mathrm{C}(16) & 119.97(17) & \mathrm{C}(23)-\mathrm{C}(22)-\mathrm{C}(21) & 118.84(18) \\ \mathrm{F}(4)-\mathrm{C}(17)-\mathrm{C}(18) & 120.83(19) & \mathrm{C}(22)-\mathrm{C}(23)-\mathrm{C}(24) & 120.29(18) \\ \mathrm{C}(16)-\mathrm{C}(17)-\mathrm{C}(18) & 119.21(18) & \mathrm{C}(23)-\mathrm{C}(24)-\mathrm{C}(19) & 122.90(18) \\ \mathrm{F}(5)-\mathrm{C}(18)-\mathrm{C}(17) & 114.79(16) & & \end{array}$


Table 7.1N. Crystal data and structure refinement for BN-6.

Identification code

Empirical formula

Formula weight

Temperature

Wavelength

Crystal system

Space group

Unit cell dimensions

Volume

Z

Density (calculated)

Absorption coefficient

$\mathrm{F}(000)$

Crystal size

Theta range for data collection

Index ranges

Reflections collected

Independent reflections

Completeness to theta $=27.13^{\circ}$

Absorption correction

Max. and min. transmission

Refinement method

Data / restraints / parameters

Goodness-of-fit on $\mathrm{F}^{2}$

Final $\mathrm{R}$ indices [I $>2 \operatorname{sigma}(\mathrm{I})]$

$\mathrm{R}$ indices (all data)

Largest diff. peak and hole pengla

C24 H20 B N

333.22

180(2) K

$0.71073 \AA$

Monoclinic

$\mathrm{P} 2(1) / \mathrm{c}$

$\mathrm{a}=12.4542(5) \AA$

$\alpha=90^{\circ}$.

$\mathrm{b}=15.7598(6) \AA$

$\beta=107.055(2)^{\circ}$.

$c=9.7650(4) \AA$

$\gamma=90^{\circ}$.

1832.35(13) $\AA^{3}$

4

$1.208 \mathrm{Mg} / \mathrm{m}^{3}$

$0.069 \mathrm{~mm}^{-1}$

704

$0.10 \times 0.10 \times 0.05 \mathrm{~mm}^{3}$

1.71 to $27.13^{\circ}$.

$-15<=\mathrm{h}<=15,-20<=\mathrm{k}<=20,-12<=\mathrm{l}<=11$

10312

$4034[\mathrm{R}(\mathrm{int})=0.0208]$

$99.5 \%$

Semi-empirical from equivalents

0.9966 and 0.9932

Full-matrix least-squares on $\mathrm{F}^{2}$

4034 / 0 / 235

1.047

$\mathrm{R} 1=0.0405, \mathrm{wR} 2=0.1012$

$\mathrm{R} 1=0.0501, \mathrm{wR} 2=0.1094$

0.305 and -0.217 e. $\AA^{-3}$ 
Table 7.1O. Bond lengths $[\AA]$ and angles $\left[^{\circ}\right]$ for BN-6.

\begin{tabular}{|c|c|c|c|}
\hline $\mathrm{B}(1)-\mathrm{C}(1)$ & $1.6200(17)$ & $\mathrm{C}(1)-\mathrm{B}(1)-\mathrm{N}(1)$ & $103.88(8)$ \\
\hline $\mathrm{B}(1)-\mathrm{C}(19)$ & $1.6237(16)$ & $\mathrm{C}(19)-\mathrm{B}(1)-\mathrm{N}(1)$ & $107.75(8)$ \\
\hline $\mathrm{B}(1)-\mathrm{C}(13)$ & $1.6275(16)$ & $\mathrm{C}(13)-\mathrm{B}(1)-\mathrm{N}(1)$ & $107.45(9)$ \\
\hline $\mathrm{B}(1)-\mathrm{N}(1)$ & $1.6396(15)$ & $\mathrm{C}(12)-\mathrm{N}(1)-\mathrm{C}(8)$ & 119.33(9) \\
\hline $\mathrm{N}(1)-\mathrm{C}(12)$ & $1.3458(14)$ & $\mathrm{C}(12)-\mathrm{N}(1)-\mathrm{B}(1)$ & $119.05(9)$ \\
\hline $\mathrm{N}(1)-\mathrm{C}(8)$ & $1.3631(14)$ & $\mathrm{C}(8)-\mathrm{N}(1)-\mathrm{B}(1)$ & 121.62(9) \\
\hline$C(1)-C(2)$ & $1.4966(16)$ & $\mathrm{C}(2)-\mathrm{C}(1)-\mathrm{B}(1)$ & $110.38(9)$ \\
\hline $\mathrm{C}(2)-\mathrm{C}(3)$ & $1.3920(18)$ & $\mathrm{C}(3)-\mathrm{C}(2)-\mathrm{C}(7)$ & $117.88(12)$ \\
\hline$C(2)-C(7)$ & $1.4015(17)$ & $\mathrm{C}(3)-\mathrm{C}(2)-\mathrm{C}(1)$ & $123.41(12)$ \\
\hline $\mathrm{C}(3)-\mathrm{C}(4)$ & $1.386(2)$ & $\mathrm{C}(7)-\mathrm{C}(2)-\mathrm{C}(1)$ & 118.71(10) \\
\hline$C(4)-C(5)$ & $1.371(2)$ & $\mathrm{C}(4)-\mathrm{C}(3)-\mathrm{C}(2)$ & $121.62(14)$ \\
\hline$C(5)-C(6)$ & $1.380(2)$ & $\mathrm{C}(5)-\mathrm{C}(4)-\mathrm{C}(3)$ & $120.10(13)$ \\
\hline$C(6)-C(7)$ & $1.4007(17)$ & $\mathrm{C}(4)-\mathrm{C}(5)-\mathrm{C}(6)$ & $119.77(13)$ \\
\hline$C(7)-C(8)$ & $1.4730(16)$ & $C(5)-C(6)-C(7)$ & $120.65(14)$ \\
\hline$C(8)-C(9)$ & $1.3939(17)$ & $C(6)-C(7)-C(2)$ & $119.93(12)$ \\
\hline $\mathrm{C}(9)-\mathrm{C}(10)$ & $1.3746(19)$ & $C(6)-C(7)-C(8)$ & $119.70(11)$ \\
\hline$C(10)-C(11)$ & $1.3807(18)$ & $C(2)-C(7)-C(8)$ & $120.32(10)$ \\
\hline$C(11)-C(12)$ & $1.3695(16)$ & $\mathrm{N}(1)-\mathrm{C}(8)-\mathrm{C}(9)$ & $119.03(11)$ \\
\hline$C(13)-C(18)$ & $1.3951(16)$ & $\mathrm{N}(1)-\mathrm{C}(8)-\mathrm{C}(7)$ & $119.02(10)$ \\
\hline$C(13)-C(14)$ & $1.4032(16)$ & $\mathrm{C}(9)-\mathrm{C}(8)-\mathrm{C}(7)$ & 121.91(10) \\
\hline$C(14)-C(15)$ & $1.3858(17)$ & $\mathrm{C}(10)-\mathrm{C}(9)-\mathrm{C}(8)$ & $120.85(11)$ \\
\hline$C(15)-C(16)$ & $1.3825(19)$ & $C(9)-C(10)-C(11)$ & $119.20(11)$ \\
\hline$C(16)-C(17)$ & $1.3798(18)$ & $C(12)-C(11)-C(10)$ & $118.27(11)$ \\
\hline$C(17)-C(18)$ & $1.3894(17)$ & $\mathrm{N}(1)-\mathrm{C}(12)-\mathrm{C}(11)$ & $123.15(11)$ \\
\hline$C(19)-C(20)$ & $1.3923(16)$ & $C(18)-C(13)-C(14)$ & $115.75(10)$ \\
\hline$C(19)-C(24)$ & $1.4002(17)$ & $\mathrm{C}(18)-\mathrm{C}(13)-\mathrm{B}(1)$ & $122.39(10)$ \\
\hline$C(20)-C(21)$ & $1.3928(16)$ & $\mathrm{C}(14)-\mathrm{C}(13)-\mathrm{B}(1)$ & $121.86(10)$ \\
\hline$C(21)-C(22)$ & $1.379(2)$ & $C(15)-C(14)-C(13)$ & $122.30(11)$ \\
\hline$C(22)-C(23)$ & $1.374(2)$ & $C(16)-C(15)-C(14)$ & $120.10(11)$ \\
\hline \multirow[t]{2}{*}{$C(23)-C(24)$} & $1.3866(18)$ & $C(17)-C(16)-C(15)$ & $119.34(11)$ \\
\hline & & $C(16)-C(17)-C(18)$ & $119.96(12)$ \\
\hline $\mathrm{C}(1)-\mathrm{B}(1)-\mathrm{C}(19)$ & $112.24(9)$ & $\mathrm{C}(17)-\mathrm{C}(18)-\mathrm{C}(13)$ & $122.54(11)$ \\
\hline$C(1)-B(1)-C(13)$ & $112.74(9)$ & $\mathrm{C}(20)-\mathrm{C}(19)-\mathrm{C}(24)$ & 115.61(11) \\
\hline$C(19)-B(1)-C(13)$ & $112.19(9)$ & $\mathrm{C}(20)-\mathrm{C}(19)-\mathrm{B}(1)$ & $124.42(10)$ \\
\hline
\end{tabular}




$\begin{array}{llll}\mathrm{C}(24)-\mathrm{C}(19)-\mathrm{B}(1) & 119.90(10) & \mathrm{C}(23)-\mathrm{C}(22)-\mathrm{C}(21) & 119.02(12) \\ \mathrm{C}(19)-\mathrm{C}(20)-\mathrm{C}(21) & 122.41(11) & \mathrm{C}(22)-\mathrm{C}(23)-\mathrm{C}(24) & 120.46(13) \\ \mathrm{C}(22)-\mathrm{C}(21)-\mathrm{C}(20) & 120.15(12) & \mathrm{C}(23)-\mathrm{C}(24)-\mathrm{C}(19) & 122.34(12)\end{array}$

\section{References}

1) Brouwer, A. M. Pure Appl. Chem. 2011, 83, 2213-2228.

2) Yang, D.-T.; Mellerup, S. M.; Wang, X.; Lu, J.-S.; Wang, S. Angew. Chem. Int. Ed. 2015, 54, 5498-5501.

3) Pelter, A.; Smith, K.; Buss, D.; Norbury, A. Tetrahedron Lett. 1991, 43, 6239-6242.

4) Abdallah, D.; Whelan, J.; Dust, J.M.; Hoz, S.; Buncel, E. J. Phys. Chem. A 2009, 113, 66406647.

5) Frisch, M. J.; Trucks, G. W.; Schlegel, H. B.; Scuseria, G. E.; Robb, M. A.; Cheeseman, J. R.; Scalmani, G.; Barone, V.; Mennucci, B.; Petersson, G. A. H.; Nakatsuji, M.; Caricato, X.; Li, H. P. F.; Hratchian, A.; Izmaylov, J.; Bloino, G.; Zheng, J. L.; Sonnenberg, M.; Hada, M.; Ehara, K.; Toyota, R.; Fukuda, J.; Hasegawa, M.; Ishida, T.; Nakajima, Y.; Honda, O.; Kitao, H.; Nakai, T.; Vreven, J. A.; Montgomery Jr., J. E.; Peralta, F.; Ogliaro, M.; Bearpark, J. J.; Heyd, E.; Brothers, K. N.; Kudin, V. N.; Staroverov, T.; Keith, R.; Kobayashi, J.; Normand, K.; Raghavachari, A.; Rendell, J. C.; Burant, S. S.; Iyengar, J.; Tomasi, M.; Cossi, N.; Rega, J. M.; Millam, M.; Klene, J. E.; Knox, J. B.; Cross, V.; Bakken, C.; Adamo, J.; Jaramillo, R.; Gomperts, R. E.; Stratmann, O.; Yazyev, A. J.; Austin, R.; Cammi, C.; Pomelli, J. W.; Ochterski, R. L.; Martin, K.; Morokuma, V. G.; Zakrzewski, G. A.; Voth, P.; Salvador, J. J.; Dannenberg, S.; Dapprich, A. D.; Daniels, O.; Farkas, J. B.; Foresman, J. V.; Ortiz, J.; Cioslowski, J.; Fox, D. J. Gaussian 09 Revision C.01, 2010.

6) Becke, A. D. J Chem Phys, 1993, 98, 5648-5652.

7) Becke, A. D. J Chem Phys, 1993, 98, 1372-1377.

8) Lee, C.; Yang, W.; Parr, R. G. Phys Rev B, 1988, 37, 785-789.

9) Goerigk, L.; Grimme, S. Phys Chem Chem Phys, 2011, 13, 6670-6688.

10) Grimme, S. J Comput Chem, 2004, 25, 1463-1473.

11) Grimme, S. J Comput Chem, 2006, 27, 1787-1799.

12) Grimme, S.; Antony, J.; Ehrlich, S.; Krieg, H. J Chem Phys, 2010, 132, 154104-154124.

13) Yanai, T.; Tew, D. P.; Handy, N. C. Chem Phys Lett, 2004, 393, 51-57.

14) Gonzalez, C.; Schlegel, H. B. J Chem Phys, 1989, 90, 2154-2161.

15) Gonzalez, C.; Schlegel, H. B. J Phys Chem, 1990, 94, 5523-5527.

16) Barone, V.; Cossi, M. J Phys Chem A, 1998, 102, 1995-2001.

17) Cammi, R.; Mennucci, B.; Tomasi, J. J Phys Chem A, 1999, 103, 9100-9108.

18) Tomasi, J.; Mennucci, B.; Cammi, R. Chem Rev, 2005, 105, 2999-3094.

19) Data used in creating the predicted UV/Vis spectra generated by GaussSum V2.2: O'Boyle, N.M.; Tenderholt, A.L.; Langner. K. M. J. Comp. Chem. 2008, 29, 839-845. 